\title{
MEASUREMENT OF NEUTRINO AND ANTINEUTRINO TOTAL CHARGED-CURRENT CROSS SECTIONS ON CARBON WITH MINERVA
}

\author{
by \\ Lu Ren \\ B.S., Nankai University, 2007 \\ M.S., Nankai University, 2010
}

Submitted to the Graduate Faculty of the Kenneth P. Dietrich School of Arts and

Sciences in partial fulfillment

of the requirements for the degree of

Doctor of Philosophy

University of Pittsburgh

2017 


\title{
UNIVERSITY OF PITTSBURGH \\ KENNETH P. DIETRICH SCHOOL OF ARTS AND \\ SCIENCES
}

\author{
This dissertation was presented \\ by \\ Lu Ren
}

It was defended on

August 29th, 2016

and approved by

Dr. Donna Naples, Professor, Department of Physics and Astromony

Dr. Arthur Kosowsky, Professor, Department of Physics and Astronomy

Dr. Vittorio Paolone, Associate Professor, Department of Physics and Astronomy

Dr. Tao Han, Professor, Department of Physics and Astronomy

Dr. Colin Morningstar, Professor, Department of Physics, Carnegie Mellon University

Dissertation Director: Dr. Donna Naples, Professor, Department of Physics and Astromony 


\title{
ABSTRACT \\ MEASUREMENT OF NEUTRINO AND ANTINEUTRINO TOTAL CHARGED-CURRENT CROSS SECTIONS ON CARBON WITH MINERVA
}

\author{
Lu Ren, PhD
}

University of Pittsburgh, 2017

This thesis presents a measurement of charged-current inclusive cross sections of muon neutrino and antineutrino interaction on carbon, and antineutrino to neutrino cross section ratio, $r$, in the energy range 2 - $22 \mathrm{GeV}$, with data collected in the MINER $\nu \mathrm{A}$ experiment. The dataset corresponds to an exposure of $3.2 \times 10^{20}$ protons on target (POT) for neutrinos and $1.0 \times 10^{20}$ POT for antineutrinos.

Measurement of neutrino and antineutrino charged-current inclusive cross sections provides essential constraints for future long baseline neutrino oscillation experiment at a few $\mathrm{GeV}$ energy range. Our measured antineutrino cross section has an uncertainty in the range $6.1 \%-10.5 \%$ and is the most precise measurement below $6 \mathrm{GeV}$ to date. The measured $r$ has an uncertainty of $5.0 \%$ - 7.5\%. This is the first measurement below $6 \mathrm{GeV}$ since Gargamelle in 1970s.

The cross sections are measured as a function of neutrino energy by dividing the efficiency corrected charged-current sample with extracted fluxes. Fluxes are obtained using the low- $\nu$ method, which uses low hadronic energy subsamples of charged-current inclusive sample to extract flux. Measured cross sections show good agreement with the prediction of neutrino interaction models above $7 \mathrm{GeV}$, and are about $10 \%$ below the model below $7 \mathrm{GeV}$. The measured $r$ agrees with the GENIE model [1] over the whole energy region. The measured cross sections and $r$ are compared with world data. 


\section{TABLE OF CONTENTS}

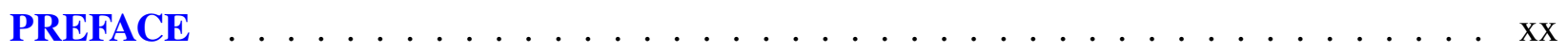

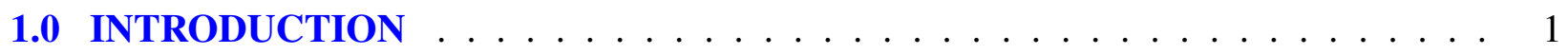

1.1 The Standard Model . . . . . . . . . . . . . . . . . . . . . . . 1

1.2 Neutrino Mixing and Oscillation $\ldots \ldots \ldots \ldots$

1.3 Neutrino Interactions $\ldots \ldots \ldots \ldots \ldots$

1.3.1 Kinematics . . . . . . . . . . . . . . . . . . . 8

1.3.2 Deep-inelastic scattering (DIS) $\ldots \ldots \ldots \ldots \ldots$

1.3.3 Resonance production (RES) . . . . . . . . . . . . . . . . . 12

1.3.4 Quasi-elastic scattering(QEL) $\ldots \ldots \ldots \ldots \ldots \ldots$

1.4 Status of Charged-current Inclusive Cross Section Measurement . . . . . . . . . 18

2.0 THE NUMI BEAM, MINER $\nu$ A DETECTOR AND MINOS NEAR DETECTOR . 22

2.1 The NuMI Beam . . . . . . . . . . . . . . . . . . . . . . . . . . 22

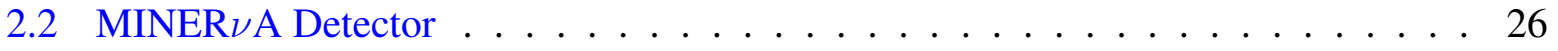

2.2 .1 Inner Detector . . . . . . . . . . . . . . . . . . . . 27

2.2.1.1 Scintillator Planes . . . . . . . . . . . . . . . . . 27

2.2.1.2 Nuclear Targets . . . . . . . . . . . . . . . . 28

2.2.1.3 Active Tracker region . . . . . . . . . . . . . . 28

2.2.1.4 Electromagnetic Calorimeter(ECAL) _ . . . . . . . . . . 29

2.2.1.5 Hadronic Calorimeter(HCAL) . . . . . . . . . . . . . . . . . 29

2.2 .2 Outer Detector . . . . . . . . . . . . . . . . . . . . . . 29

2.2 .3 Light Collection . . . . . . . . . . . . . . . . . 30

2.2 .4 Data Acquisition . . . . . . . . . . . . . . . . 30 
2.2 .5 Calibration . . . . . . . . . . . . . . . 31

2.2.5.1 Ex situ calibrations $\ldots \ldots \ldots \ldots \ldots \ldots$

2.2.5.2 In situ calibrations . . . . . . . . . . . . . . . 33

2.2.5.3 Absolute energy scale . . . . . . . . . . . . . . 33

2.2.5.4 Hadron energy scale . . . . . . . . . . . . . . . . . 34

2.3 MINOS Near Detector . . . . . . . . . . . . . . . . . . . . . . . 35

3.0 GENERATOR MODELS $\ldots \ldots \ldots \ldots \ldots \ldots \ldots$

3.1 GENIE Hybrid $\ldots \ldots \ldots \ldots \ldots \ldots$

3.1 .1 GENIE $2.8 .4 \ldots \ldots \ldots \ldots \ldots$

3.1.1.1 Quasi-Elastic Scattering (QEL) . . . . . . . . . . . . . . 39

3.1.1.2 Baryon Resonance Production (RES) . . . . . . . . . . . . 40

3.1.1.3 Deep Inelastic Scattering (DIS) _ . . . . . . . . . . . . 40

3.1.1.4 Other Contributions . . . . . . . . . . . . . . . . 41

3.1.1.5 Cross section model tuning . . . . . . . . . . . . 43

3.1.2 Hybrid Only Model Components . . . . . . . . . . . . . . . . . . 44

3.1.2.1 Random Phase Approximation (RPA) . . . . . . . . . . . . . 44

3.1 .2 .2 MEC . . . . . . . . . . . . . . . . 44

3.1.2.3 Pion Reweighting . . . . . . . . . . . . . . . . . 48

3.1.3 Final State Interaction (FSI) Model $\ldots \ldots \ldots \ldots$

3.2 NuWro . . . . . . . . . . . . . . . . . . . . . . . . . . . . . . 49

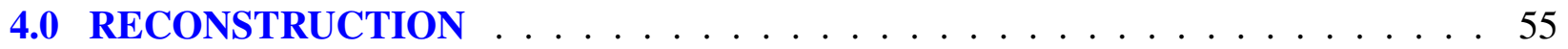

4.1 Cluster Formation . . . . . . . . . . . . . . . . 56

4.2 Track Reconstruction ． . . . . . . . . . . . . . . . . . . 57

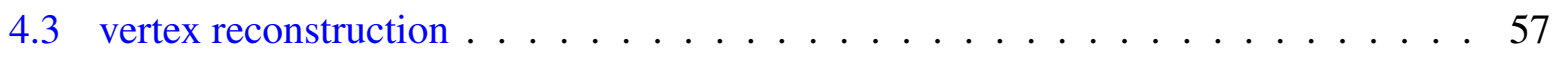

4.4 Charged-current event reconstruction $\ldots \ldots \ldots \ldots \ldots$

4.5 Recoil System Reconstruction . . . . . . . . . . . . . . . . . . . . 58

4.6 Reconstructed Event Corrections _. . . . . . . . . . . . . . . . . . 59

4.6.1 Shower Cleaning Corrections _. . . . . . . . . . . . . . . . . 59

4.6.1.1 Cylinder Region . . . . . . . . . . . . . . . . . . 61

4.6.1.2 Non-cylinder Region _ . . . . . . . . . . . . . . . 62 
4.6 .2 Calorimetry Fix $\ldots \ldots \ldots \ldots$

4.6 .3 Vertex Correction . . . . . . . . . . . . . . . . . . . . 64

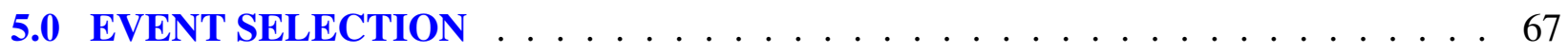

5.1 Data Sample . . . . . . . . . . . . . . . . . . . . . 67

5.2 MINOS Subsample Study . . . . . . . . . . . . . . . . . . 74

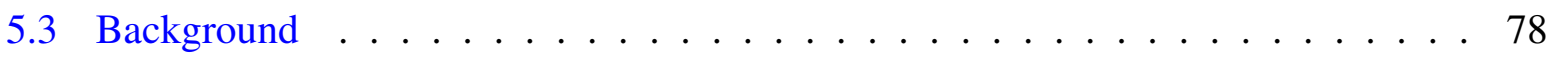

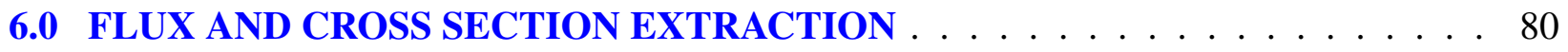

6.1 Low- $\nu$ Flux Measurement Technique . . . . . . . . . . . . . . . . 80

6.2 Cross Section and Flux Extraction . . . . . . . . . . . . . . . . 82

6.3 Generator level model-dependent corrections . . . . . . . . . . . . . 85

6.3 .1 Low- $\nu$ correction . . . . . . . . . . . . . . . . . . 85

6.3 .2 Kinematic Correction . . . . . . . . . . . . . 86

6.3 .3 Isoscalar correction $\ldots \ldots \ldots \ldots \ldots$

6.4 NuWro-based model-dependent Corrections $\ldots \ldots \ldots 8$

6.5 Flux Normalization $\ldots \ldots \ldots$. . . . . . . . . . . . . . . 89

6.5.1 Antineutrino Cross Section Normalization _ . . . . . . . . . . 91

6.5.2 External Cross Section Measurements between 12 and $22 \mathrm{GeV}$ (normalization bin). . . . . . . . . . . . . . . . . . . . . . . 92

7.0 SYSTEMATIC UNCERTAINTY . . . . . . . . . . . . . . . . . . . . . 107

7.1 Normalization . . . . . . . . . . . . . . . . . . . . . 107

7.2 Reconstruction . . . . . . . . . . . . . . . . . . . 108

7.2 .1 Muon Energy Scale . . . . . . . . . . . . . . . . . . . . . 108

7.2.2 Hadron Energy Scale . . . . . . . . . . . . . . . . . . . . . 109

7.2.3 Shower Cleaning Correction . . . . . . . . . . . . . . . . . . 114

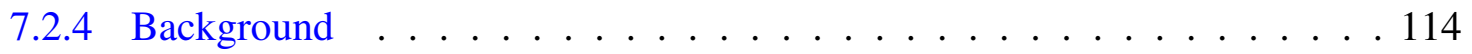

7.3 Final State Interaction Uncertainties $\ldots \ldots \ldots$

7.4 Cross Section model uncertainties _ . . . . . . . . . . . . . 125

7.4.1 GENIE reweightable uncertainties . . . . . . . . . . . . . . 125

7.4 .2 RPA . . . . . . . . . . . . . . . . . . . . . 134

$7.4 .32 \mathrm{2p} 2 \mathrm{~h}$ Model Uncertainty . . . . . . . . . . . . . . . . . . 134 
7.5 Summary of Systematic Uncertainties . . . . . . . . . . . . . . . . . . . . 141

8.0 RESULTS . . . . . . . . . . . . . . . . . . . . . . . . . . . . . 146

8.1 Cross sections and the ratio $\ldots \ldots \ldots . \ldots \ldots$

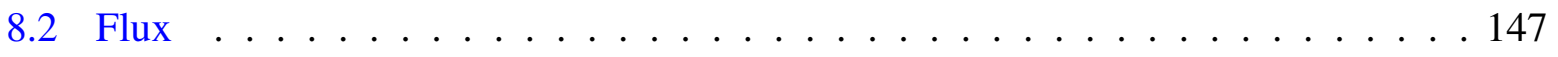

8.3 Comparison of data with MC model . . . . . . . . . . . . . . . . . . . 149

8.4 Cross Check and additional studies . . . . . . . . . . . . . . 166

8.4 .1 NuWro-based results . . . . . . . . . . . . . . . . 166

8.4.2 Extract cross sections with low- $\nu$ flux reweighted $\ldots \ldots \ldots$. . . . . 166

8.4 .3 Extract cross sections in opposite modes . . . . . . . . . . 168

8.4.4 Extract cross sections in different regions of detector $\ldots \ldots \ldots$. . . . 168

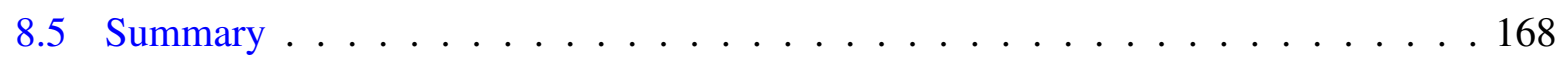

9.0 CONCLUSION . . . . . . . . . . . . . . . . . . . . . . . . . . 172

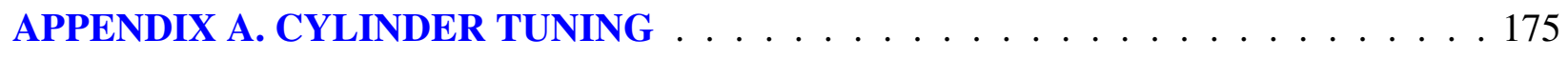

APPENDIX B. COVARIANCE MATRICES OF MEASURED QUANTITIES . . . . . . 179

B.1 Covariance matrix . . . . . . . . . . . . . . . . . . . . 179

B.1.1 Definition . . . . . . . . . . . . . . . . . . . . 179

B.1.2 Covariance Matrix . . . . . . . . . . . . . . . 180

B.1.3 Components of Covariance Matrix . . . . . . . . . . . 180

B.2 Correlation Matrix . . . . . . . . . . . . . . . . . 186

B.3 $\chi^{2}$ Calculation for Cross Sections and the Ratio . . . . . . . . . . . . . 193

APPENDIX C. CODE OF CALCULATING CROSS SECTIONS FROM TABLES . . . 194

BIBLIOGRAPHY . . . . . . . . . . . . . . . . . . . . . . . . . . 199 


\section{LIST OF TABLES}

1 Properties of leptons. . . . . . . . . . . . . . . . . . . 2

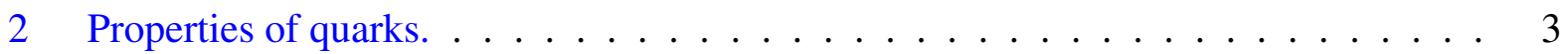

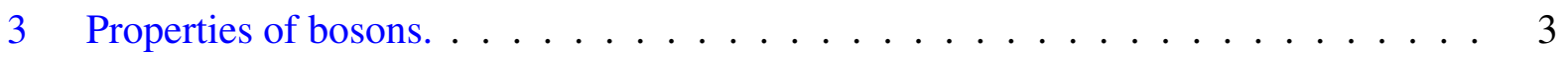

4 The best-fit values and $3 \sigma$ allowed ranges of the 3-neutrino oscillation parameters, derived from a global fit of the current neutrino oscillation data. $\Delta m_{3 l}^{2}=\Delta m_{31}^{2}>0$ for normal hierarchy (NH), $\Delta m_{3 l}^{2}=\Delta m_{32}^{2}<0$ for inverted hierarchy (IH) (Taken

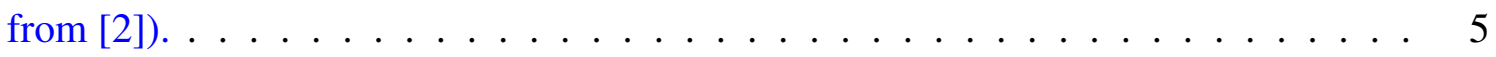

5 Parameters for QEL model in GENIE 2.8.4 . . . . . . . . . . . . . . . . . . 39

6 Constants in the Bodek-Yang (BY) DIS structure function model and in the BodekYang GRV-LO-98 PDF used in GENIE v2.8.4 . . . . . . . . . . . . . . . . . . . 41

$7 \quad 2 \mathrm{p} 2 \mathrm{~h}$ contributions in generated sample at $E_{\nu}=2.5 \mathrm{GeV} \ldots \ldots . \ldots$

8 Parameters used in NuWro generator. . . . . . . . . . . . . . . . 50

9 Reconstructed event corrections. . . . . . . . . . . . . . . . . 60

10 Muon purity used in the cylinder correction. . . . . . . . . . . . . . 65

11 Effect of event selection cuts for both FHC (minerva13C only) and RHC data and simulated samples. . . . . . . . . . . . . . . . . . . 69

12 Value of $\nu_{o}$ for each energy range. . . . . . . . . . . . . . . 73

13 Measured neutrino cross section and flux sample yields corrected for wrong-sign and neutral current background contaminations. . . . . . . . . . . . . . 93

14 Antineutrino measured cross section and flux sample rates corrected for wrong sign and neutral current background contaminations. . . . . . . . . . . . . . . . 94

15 Neutrino raw data in 12-22 GeV for different flux samples. . . . . . . . . . . . 95 
16 Antineutrino raw data in $12-22 \mathrm{GeV}$ for different flux samples. . . . . . . . . . . 96

17 Effect of kinematic cuts. . . . . . . . . . . . . . . . . . . . . 97

18 Composition of scintillator planes, by mass percentage. . . . . . . . . . . . 97

19 Neutrino cross section model dependent corrections computing using GENIE hybrid. 98

20 Neutrino cross section model dependent corrections computed using NuWro. . . . . 98

21 Antineutrino cross section model dependent corrections computed using GENIE hybrid. . . . . . . . . . . . . . . . . . . . . . . . . 999

22 Antineutrino cross section model dependent corrections computed using NuWro. . . 99

23 GENIE-based $G_{\text {corr }}\left(\nu_{0}\right)$ correction for different $\nu$ cut. . . . . . . . . . . 100

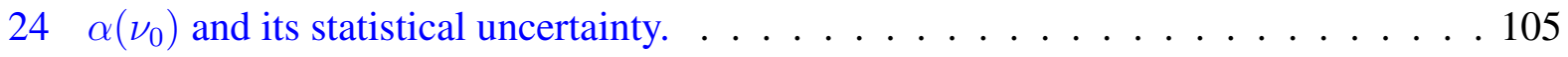

25 World isoscalar neutrino cross section data at $12-22 \mathrm{GeV} . \ldots . \ldots 106$

26 Summary of normalization related uncertainties. . . . . . . . . . . . 108

27 Components of muon energy scale. . . . . . . . . . . . . . . . . . . . . 109

28 Hadron energy scale uncertainty components. . . . . . . . . . . . . . . 110

29 Breakdown of hadron energy scale uncertainty for neutrino cross section. . . . . . 110

30 Breakdown of hadron energy scale uncertainty for antineutrino cross section. . . . . 111

31 Breakdown of hadron energy scale uncertainty for the ratio, $r \ldots \ldots \ldots \ldots$

32 Final state interaction uncertainties(reweightables) implemented in GENIE. . . . . . 118

33 Breakdown of FSI uncertainty for the neutrino cross section. . . . . . . . . . . . 119

34 Breakdown of FSI uncertainty for the antineutrino cross section. . . . . . . . . . 120

35 Breakdown of FSI uncertainty for the ratio, $r \ldots \ldots \ldots \ldots \ldots \ldots \ldots \ldots \ldots$

36 Final state interaction uncertainties (non-reweightables) not implemented in GENIE

37 Cross section model uncertainties considered [1] . . . . . . . . . . . . 126

38 Breakdown of cross section model uncertainty for neutrino cross section. . . . . . . 127

39 Breakdown of cross section model uncertainty for antineutrino cross section. . . . . 128

40 Breakdown of cross section model uncertainty for the ratio, $r \ldots \ldots$. . . . . . 129

41 Breakdown of cross section model uncertainty for neutrino flux. . . . . . . . . . 130

42 Breakdown of cross section model uncertainty for antineutrino flux. . . . . . . . 131

43 Systematic uncertainty breakdown for the neutrino cross section. . . . . . . . . 143 
44 Systematic uncertainty breakdown for the antineutrino cross section. . . . . . . . . 144

45 Systematic uncertainty breakdown for the ratio, $r \ldots \ldots$. . . . . . . . 144

46 The ratio, $r$, and its statistical, systematic and total error. Errors are fractional. . . . 152

47 Neutrino cross section its statistical, systematic and total error. Errors are fractional. 153

48 Antineutrino cross section its statistical, systematic and total error. Errors are fractional. . . . . . . . . . . . . . . . . . . . 154

49 Neutrino flux in unit of neutrinos $/ \mathrm{GeV} / \mathrm{m}^{2} / 10^{6}$ pot and its statistical, systematic and total error. Errors are fractional. . . . . . . . . . . . . . . 154

50 Antineutrino flux in unit of neutrinos $/ \mathrm{GeV} / \mathrm{m}^{2} / 10^{6} \mathrm{pot}$ and its statistical, systematic and total error. Errors are fractional. . . . . . . . . . . . . 155

51 MINOS tracking efficiency correction. . . . . . . . . . . . . 157

52 Covariance matrix corresponding to total error for the extracted neutrino cross section in the FHC and antineutrino cross section in the RHC beam mode. It includes statistical error and all systematic errors, except for the external normalization uncertainty. The bin boundaries are in units of $\mathrm{GeV}$. The covariance elements are in units of $\left(10^{-38} \mathrm{~cm}^{2} / \mathrm{GeV}\right)^{2}$ and scaled by a factor of 1000 . Numbers are obtained from Fig. 114. . . . . . . . . . . . . . . . . . . . . . 182

53 Covariance matrix corresponding to systematic error for the extracted neutrino cross section in the FHC and antineutrino cross section in the RHC beam mode. The bin boundaries are in units of GeV. The covariance elements are in units of $\left(10^{-38} \mathrm{~cm}^{2} / \mathrm{GeV}\right)^{2}$ and scaled by a factor of $1000 \ldots \ldots \ldots \ldots$

54 Covariance matrix of extracted cross section ratio, $r$, scaled by 1000 . It includes statistical error and all systematic errors. . . . . . . . . . . . . . . . . . 184

55 Correlation matrix corresponding to total error for the extracted neutrino cross section in the FHC and antineutrino cross section in the RHC beam mode. It includes statistical and systematic errors. The bin boundaries are in units of GeV. . . . . . 191

56 Correlation matrix corresponding to systematic error for the extracted neutrino cross section in the FHC and antineutrino cross section in the RHC beam mode. It includes statistical and systematic errors. The bin boundaries are in units of GeV. 
57 Calculated $\chi^{2}$ for neutrino and antineutrino cross sections and the ratio, $r$, between the GENIE-Hybrid model and the data. . . . . . . . . . . . . . . . . 193 


\section{LIST OF FIGURES}

1 Left shows the $\nu_{e}$ appearance probability as a function of neutrino energy at $1300 \mathrm{~km}$ for different $\delta$ values for neutrinos for normal hierarchy. Right shows simulated energy distribution of DUNE events assuming normal hierarchy, $\delta_{C P}=0$ and a 150 kt. MW · year exposure in the neutrino-beam mode (Both taken from [3]). . . . . . . 7

2 Feynman diagram of $\nu_{\mu} N \mathrm{CC}$ interaction. . . . . . . . . . . . . 9

3 An example of charged-current deep-inelastic scattering. . . . . . . . . . . . . 10

4 An example of charged-current single pion production. . . . . . . . . . . . . . 13

5 An example of charged-current quasi-elastic scattering. . . . . . . . . . . 16

6 Neutrino (left) and antineutrino (right) total charged-current cross sections and the components of QEL, DIS and RES． . . . . . . . . . . . . . . . . . 18

7 Measurements of $\nu_{\mu}$ and $\overline{\nu_{\mu}} \mathrm{CC}$ inclusive scattering cross sections divided by neutrino energy as a function of neutrino energy (Taken from [4]) . . . . . . . . . . . . 19

8 Measurements of antineutrino to neutrino charged-current cross section ratio. . . . . 21

9 Schematic of the NuMI Beam(taken from [5] ) . . . . . . . . . . . 23

10 A illustration of two possible trajectories inside two magnetic horns. . . . . . . . 23

11 A illustration of forward horn current (top) and reverse horn current (bottom) modes (taken from [6]). . . . . . . . . . . . . . . . . . 24

12 A sketch of the area downstream of the decay pipe, which includes the hadron absorber, the hadron monitor, and the three muon monitors (taken from [5]). . . . . 24

13 A illustration of six time batches structure from MINOS online monitoring page. . . 25

14 Predicted fluxes in FHC and RHC beam modes obtained using the "PPFX gen-2 thin" flux described in [7] . . . . . . . . . . . . . . . . 26 
15 Front view of MINER $\nu$ A detector, taken from [8]. . . . . . . . . . . . . 27

16 Side view of MINER $\nu$ A detector, showing the nuclear target region, tracker region, surrounding calorimeters and MINOS ND, taken from [8]. . . . . . . . . . 28

17 From left to right are $\mathrm{X}, \mathrm{U}$ and $\mathrm{V}$ scintillator plane orientations. The lines show the direction of scintillator strips, taken from [8]. . . . . . . . . . . . . . . . 29

18 Schematic of a single optical channel in MINER $\nu$ A, taken from [8]. . . . . . . 32

19 Absolute energy scale calibration using MEU. . . . . . . . . . . . . . . . . 34

20 Schematic of the MINOS ND. . . . . . . . . . . . . . . . . 35

21 Left shows $P_{\text {curv }}-P_{\text {range }}$ for inclusive events with muon stops in the calorimeter of MINOS, right shows $1 / P_{\text {curv }}-1 / P_{\text {range }}$ for the same events (taken from [8]). . . 37

$22 Q^{2}$ vs hadron energy distributions for DIS, RES and QEL, obtained from MC sample generated with GENIE $2.8 .4 \ldots \ldots \ldots$

23 Set of irreducible diagrams responsible fore RPA effect in $1 \mathrm{p} 1 \mathrm{~h}$ contribution to Wself energy, taken from [9]. . . . . . . . . . . . . . . . . . 45

24 Two-dimensional correction in (q0, q3) which is formed from the ratio of cross sections between the model with and without RPA effect. . . . . . . . . . . 46

25 Feynman diagram for $2 \mathrm{p} 2 \mathrm{~h}(\mathrm{MEC})$ interaction. . . . . . . . . . . . . . . . 47

26 Neutrino and antineutrino CC differential cross section in oxygen, at 60 degrees of scattering angle and neutrino energy at $0.75 \mathrm{GeV}$, taken from [10]. . . . . . . 47

27 Hadron energy distributions of generated MC neutrino events with $E_{\nu}=2.5 \mathrm{GeV}$ for neutrino (top left) and antineutrino (top right) and muon energy distributions of those events for neutrino (bottom left) and antineutrino (bottom right). Nominal is from GENIE v2.8.4 model, $2 \mathrm{p} 2 \mathrm{~h}$ is from Nieves model. . . . . . . . . . . . . . 49

28 Inclusive neutrino cross section predicted by NuWro, taken from [11] . . . . . . 52

29 Inclusive antineutrino cross section predicted by NuWro, taken from [11]. . . . . 53

30 Transverse enhancement ratio as a function of $Q^{2}$, taken from [12] . . . . . . . . 54

31 The display of a neutrino interaction event, with a vertex and three tracks recon-

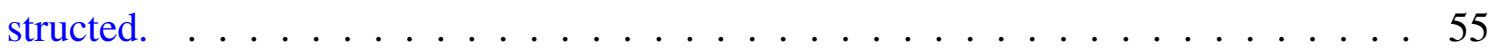

32 Time slices within the MINERvA Run 2160 Subrun 1 Gate 594 . . . . . . . . . . 56 
$33 \delta E_{i}^{s h}$ distributions in subdetector $i$ ( $i=$ tracker, ecal, or hcal) for rock muon sample of data(left) and MC(right). . . . . . . . . . . . . . . . 61

34 An example of a muon track starting in the tracker. . . . . . . . . . . . . . . 62

35 Effect of shower cleaning correction on hadron energy in $E_{\nu}=(2,3) \mathrm{GeV}$ for neutrino (top) and antineutrino (bottom) data and MC . . . . . . . . . . . . . . 66

36 MINER $\nu$ A Low Energy data starting from November 2009. . . . . . . . . . . . . 68

37 Cut efficiency as a function of reconstructed neutrino energy for FHC neutrino data (left) and simulated (right) samples. . . . . . . . . . . . . . . . . . 70

38 Inclusive Sample of FHC neutrinos with and without all cuts. (39\% of FHC data is used for this figure.) . . . . . . . . . . . . . 70

39 Cut efficiency as a function of reconstructed neutrino energy for RHC antineutrino data $($ left $)$ and simulated samples. . . . . . . . . . . . . . . 71

40 Inclusive Sample of RHC neutrinos with and without all cuts. . . . . . . . . . . 71

41 Cross section and flux samples for FHC neutrino(left) and RHC antineutrino(right). 72

42 Ratio of flux samples to inclusive sample for FHC neutrino(left) and RHC antineu-

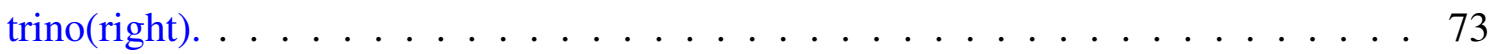

43 Muon track end distributions of four MINOS subsamples. . . . . . . . . . . . 75

44 FHC inclusive neutrino sample divided into four subsamples in MINOS ND. . . . . 76

45 Reconstruction efficiency of different MINOS subsamples for inclusive sample of FHC neutrinos. . . . . . . . . . . . . . . . 76

46 RHC inclusive antineutrino sample divided into four subsamples in MINOS ND. . . 77

47 Reconstruction efficiency of different MINOS subsamples for inclusive sample of RHC antineutrinos. . . . . . . . . . . . . . . . . . 77

48 Neutral-current contamination of cross section and flux samples for FHC neutrino (left) and RHC antineutrino (right). . . . . . . . . . . . . . . 78

49 Wrong-sign contamination of cross section and flux samples for FHC neutrino(left) and RHC antineutrino(right). . . . . . . . . . . . . . . . . 79 
50 (Left) Distribution of true muon energy for generated and reconstructed events. (Right) Distribution of true muon angle w.r.t beam for generated and reconstructed events, both with an minimal true muon energy cut of $1.8 \mathrm{GeV}$ applied. Top figures show neutrino samples for all neutrino energies. Bottom figures show antineutrino samples for all neutrino energies. . . . . . . . . . . . . . . . . . . . 84

$51 A_{C C}^{\mathrm{DET}}$ for cross section and $A_{\Phi}\left(E_{i}\right)$ for flux samples of FHC neutrinos (left) and RHC antineutrinos (right) . . . . . . . . . . . . . . . 85

52 Kinematic corrections for FHC neutrino(left) and RHC antineutrino(right). . . . . 100

53 GENIE-based (solid curves) and NuWro-based (dots) low- $\nu$ correction for neutrinos (upper) and antineutrinos (lower) . . . . . . . . . . . . . . 101

54 Hadron energy distributions and their breakdown in the energy region $2<E_{\nu}<$ $3 \mathrm{GeV}$ of simulated GENIE (left) and NuWro (right) samples for neutrino. Plots on top show all generated events, while the bottom ones show events which pass kinematic cuts. . . . . . . . . . . . . . . . . . 102

55 Hadron energy distributions and their breakdown in the energy region $2<E_{\nu}<$ $3 \mathrm{GeV}$ of simulated GENIE (left) and NuWro (right) samples for antineutrino. Plots on top show all generated events, while the bottom ones show events which pass kinematic cuts. . . . . . . . . . . . . . . . . 103

56 GENIE-based and NuWro-based isoscalar corrections for neutrinos and antineutrinos.104

57 Extracted antineutrino cross sections using different $\nu$ cuts. Error bars are statistical errors only. . . . . . . . . . . . . . . . . . . 105

58 World measurements of CC inclusive neutrino cross section between 12 and $22 \mathrm{GeV}$. 106

59 Breakdown of hadron energy scale uncertainties for neutrino cross section. . . . . . 112

60 Breakdown of hadron energy scale uncertainties for antineutrino cross section. . . . 112

61 Breakdown of hadron energy scale uncertainties for cross section ratio $r$. . . . 113

62 Effect of shower cleaning corrections on cross sections. . . . . . . . . . . . 114

63 Effect of shower cleaning corrections on cross section ratio $r \ldots \ldots 15$

64 Low- $\nu$ flux reweighting function for RHC neutrinos. . . . . . . . . . . . . 116

65 Shifted / nominal neutrino (left) and antineutrino (middle) cross sections and $r$ (right) due to non-reweightable FSI uncertainties. . . . . . . . . . . . . 117 
66 Size of GENIE-based FSI model uncertainty for neutrino cross section. . . . . . . . 122

67 Size of GENIE-based FSI model uncertainty for antineutrino cross section. . . . . . 122

68 Size of GENIE-based FSI model uncertainty for cross section ratio $r$. . . . . . . 123

69 FSI uncertainty of extracted FHC neutrino flux. . . . . . . . . . . . . . 123

70 FSI uncertainty of extracted RHC antineutrino flux. . . . . . . . . . . . . . . 124

71 Cross section model uncertainty for the neutrino cross section. . . . . . . . . . 132

72 Cross section model uncertainty for the antineutrino cross section. . . . . . . . . 132

73 Cross section model uncertainty for the ratio, $r \ldots \ldots \ldots$

74 Cross section model uncertainty of extracted FHC neutrino flux. It shows the sum of contributions from each component and three components with the largest con-

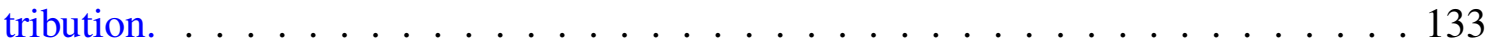

75 Cross section model uncertainty of extracted RHC antineutrino flux. It shows the sum of contributions from each component and three components with the largest

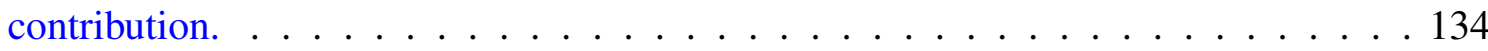

76 The double-differential cross section in six regions of $\mathrm{q} 3$ is compared to the GENIE 2.8.4 model with RPA suppression (long-dashed), and then combined with a QElike 2 p2h component (solid). (Taken from [13]) . . . . . . . . . . . . . 135

$772 \mathrm{p} 2 \mathrm{~h}$ reweighting function accounting for the data MC discrepancy shown in Fig. 76. (Taken from $[14]) \ldots \ldots \ldots \ldots \ldots \ldots \ldots \ldots$

78 Effect of the 2-dimensional fitting in Fig. 77 on available energy distribution. . . . . 137

79 Remaining difference in hadron energy distributions between data and simulation after applying the 2-dimensional fitting for neutrino (left) and antineutrino (right)

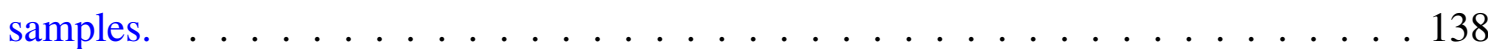

80 Data MC comparison of hadron energy after with and without data-driven fits. Left is for neutrino, Right is for antineutrino . . . . . . . . . . . . . . . . 139

81 Effect of $2 \mathrm{p} 2 \mathrm{~h}$ reweighting on neutrino cross section . . . . . . . . . . . . . . 139

82 Effect of $2 \mathrm{p} 2 \mathrm{~h}$ reweighting on antineutrino cross section. . . . . . . . . . . . . 140

83 Effect of $2 \mathrm{p} 2 \mathrm{~h}$ reweighting on the ratio, $r \ldots \ldots \ldots \ldots \ldots$

84 Error summary for neutrino cross section. External uncertainty is shown as the normalization curve. . . . . . . . . . . . . . . . . 141 
85 Error summary for antineutrino cross section. External uncertainty and $G_{\text {corr }}\left(\nu_{0}\right)$ uncertainty are added in quadrature and shown as the normalization curve. . . . . . 142

86 Error summary for the ratio, $r . G_{c o r r}\left(\nu_{0}\right)$ uncertainty is shown as the normalization

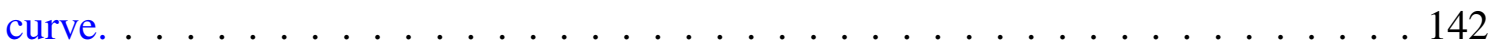

87 Error summary ofor FHC neutrino flux. External uncertainty is shown as the normalization curve. . . . . . . . . . . . . . . . . . . . 145

88 Error summary for RHC antineutrino flux. External uncertainty and $G_{c o r r}\left(\nu_{0}\right)$ uncertainty are added in quadrature and shown as the normalization curve. . . . . . . . 145

89 Measured neutrino cross section using GENIE-Hybrid corrections (solid points), comparing with GENIE Hybrid model curve. . . . . . . . . . . . . . . . . . . . . 147

90 Measured antineutrino cross section using GENIE-Hybrid corrections (solid points), comparing with GENIE Hybrid model curve. . . . . . . . . . . . . . . . . . . . . 148

91 Measured cross section ratio, $r$, using GENIE-Hybrid corrections (solid points), comparing with GENIE Hybrid model curve. . . . . . . . . . . . . . . . . . . . . . 149

92 Statistical and systematic errors comparison of neutrino cross section. . . . . . . 150

93 Statistical and systematic errors comparison of antineutrino cross section. . . . . . 150

94 Statistical and systematic errors comparison of the ratio, $r \ldots \ldots \ldots 151$

95 Measured FHC neutrino flux comparing with simulated MC flux. Error bars include statistical error and systematic uncertainty. . . . . . . . . . . . . . 156

96 Measured RHC antineutrino flux comparing with simulated MC flux. Error bars include statistical error and systematic uncertainty. . . . . . . . . . . . . . 157

97 Low- $\nu$ flux reweighting function. Error bar includes statistical error as well as systematic uncertainties of extracted flux from data. . . . . . . . . . . . . . 158

98 Comparison of data and MC simulation distributions of neutrino energy for FHC neutrinos (left) and RHC antineutrinos(right) . . . . . . . . . . . . . . . . . 159

99 Comparison of data and MC simulation distributions of muon energy for FHC neutrinos (left) and RHC antineutrinos(right) . . . . . . . . . . . . . 160

100 Comparison of data and MC simulation distributions of muon angle w.r.t beam for FHC neutrinos (left) and RHC antineutrinos(right) . . . . . . . . . . . . . . 161 
101 Comparison of data and MC simulation distributions of hadronic energy with $2<$ $E_{\nu}<3 \mathrm{GeV}$ (top) and $3<E_{\nu}<4 \mathrm{GeV}$ (bottom) for FHC neutrinos (left) and RHC antineutrinos(right). . . . . . . . . . . . . . . . . 162

102 Comparison of data and MC simulation distributions of hadronic energy with $4<$ $E_{\nu}<5 \mathrm{GeV}$ (top) and $5<E_{\nu}<7 \mathrm{GeV}$ (bottom) for FHC neutrinos (left) and RHC antineutrinos(right). . . . . . . . . . . . . . . . . 163

103 Comparison of data and MC simulation distributions of hadronic energy with $7<$ $E_{\nu}<12 \mathrm{GeV}$ (top) and $12<E_{\nu}<22 \mathrm{GeV}$ (bottom) for FHC neutrinos (left) and RHC antineutrinos(right). . . . . . . . . . . . . . . . 164

104 Comparison of data and MC simulation distributions of hadronic energy with $2<$ $E_{\nu}<22 \mathrm{GeV}$ for FHC neutrinos (left) and RHC antineutrinos(right). . . . . . . . 165

105 Measured neutrino (top) and antineutrino (bottom) cross sections with low- $\nu$ flux reweighted, comparing with the nominal on the left, the ratio of reweighted to nominal neutrino cross section on the right. Error bars are statistical error only. . . . . . 167

106 Measured neutrino cross section in RHC mode (top) and antineutrino cross section in FHC mode (bottom), comparing with the nominal on the left, the ratio of opposite mode to nominal neutrino cross section on the right. Error bars are statistical error only. . . . . . . . . . . . . . . . . . . . . 169

107 Wrong-sign contamination for FHC antineutrino (left) and RHC neutrino (right)

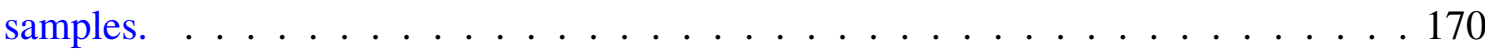

108 Measured neutrino (top) and antineutrino (bottom) cross sections from left and right of MINOS near detector on the left, ratio of left to right on the right. Error bars are statistical error only. . . . . . . . . . . . . . . . . . . . 171

109 Extracted neutrino and antineutrino cross sections, compared with world data. . . . 173

110 Extracted cross section ratio, $r$, compared with world data. . . . . . . . . . 174

111 Muon purity and hadron completeness for different radii in the tracker region. . . . 177

112 Muon purity and hadron completeness in tracker with $d=300 \mathrm{~mm}$. . . . . . . . . 177

113 Muon purity and muon completeness for different radii in Ecal and Hcal. . . . . . 178

114 Covariance matrix for the extracted neutrino cross section in the FHC and antineutrino cross section in the RHC beam mode. . . . . . . . . . . . . . . . . 181 
115 Statistical error component of Covariance matrix for the extracted neutrino cross section in the FHC and antineutrino cross section in the RHC beam mode. The bin boundaries are in units of $\mathrm{GeV}$. The covariance elements are in units of $\left(10^{-38} \mathrm{~cm}^{2} / \mathrm{GeV}\right)^{2}$ and scaled by a factor of 1000. Index 1-9 are for neutrino cross section and 10-18 are for antineutrino cross section. . . . . . . . . . . . . . . . 185

116 Components coming from statistical error for the ratio. The bin boundaries are in units of GeV. The covariance elements are scaled by a factor of 1000 . . . . . . . 186

117 GENIE cross section model uncertainty component of Covariance matrix for the extracted neutrino cross section in the FHC and antineutrino cross section in the RHC beam mode. . . . . . . . . . . . . . . . . . . . . . . 187

118 GENIE FSI uncertainty component of Covariance matrix for the extracted neutrino cross section in the FHC and antineutrino cross section in the RHC beam mode. . . 188

119 Hadron energy scale component of Covariance matrix for the extracted neutrino cross section in the FHC and antineutrino cross section in the RHC beam mode. . . 189

120 Components coming from GENIE cross section model uncertainty for the ratio. The bin boundaries are in units of $\mathrm{GeV}$. The covariance elements are scaled by a factor

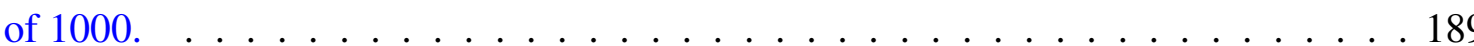

121 Components coming from GENIE FSI uncertainty for the ratio. The bin boundaries are in units of GeV. The covariance elements are scaled by a factor of 1000 . . . . 190

122 Components coming from muon energy scale for the ratio. The bin boundaries are in units of GeV. The covariance elements are scaled by a factor of 1000 . . . . . . . 190 


\section{PREFACE}

First, I would like to thank my advisor, Dr. Donna Naples, for her patient guidance and support on my work through the last five years. I cannot forget how many times she stayed up late at night helping me with my talks and how much of her family time I had stolen from Arlo and Max. I was so lucky to have her as my advisor. I would not be able to finish this work without her help and encouragement.

Many thanks to the MINER $\nu$ A collaboration, especially Debbie Harris, Kevin McFarland, Jeff Nelson, Jorge Morfin, Heather Ray, Mike Kordosky, Gabe Perdue and Laura Fields for all the discussions and suggestions on my analysis. I would also like to thank Howard Budd, Dan Ruggiero, Leo Bellantoni, Steve Hahn and Carrie McGivern for all the guidance on hardware work. Thanks to Steve Dytman, Istvan Danko and Hugh Gallagher for their help on GENIE-related work. I also got help from a lot of people, Richard Gran, Phil Rodrigues, Trung Le, Jeremy Wolcott, Brian Tice, Brandon Eberly, Leo Aliaga, Minerba Betancourt, Josh Devan, Mousumi Datta, Marianette Wospakrik, Joel Mousseau, Dipak Rimal and Dun Zhang, many thanks to all of you.

I also appreciate that I have so many good friends both at Pitt and Fermilab, as well as my dearest friends, Xinrui, Jing and Chen, you girls always cheered me up.

At the end, I would like to thank my parents for their love and support. I am nothing without you. 


\subsection{INTRODUCTION}

Neutrino oscillation experiments have proved that neutrinos have non-zero mass, which can not be explained by the Standard Model of particle physics. Cross section measurements of neutrino and antineutrino in a few $\mathrm{GeV}$ energy region play an important role in determining $\mathrm{CP}$-violating phase and mass hierarchy in future long-baseline neutrino oscillation experiments. Many experiments have measured neutrino cross section below $20 \mathrm{GeV}$. However, the antineutrino cross section and the antineutrino to neutrino cross section ratio, $r$, in the relevant energy region are poorly measured, which limits the precision of oscillation experiments. A detailed review of those cross section and ratio measurements is presented in Sec. 1.4.

In this chapter, we describe neutrino mixing and oscillation, long baseline neutrino oscillation experiment, neutrino charged-current interaction models and the status of inclusive cross section measurements. Chapter 2 describes the neutrino beam, MINER $\nu$ A and MINOS Near Detector. Chapter 3 describes the simulation. Chapter 4 presents the event reconstruction from raw data. Chapter 5 discusses the data sample and event selection. Chapter 6 discusses the method of flux and cross section extraction. Chapter 7 describes the evaluation of systematic uncertainties. Chapter 8 gives the results of measured fluxes, cross sections and the ratio. Chapter 9 compares our results with previous measurements.

\subsection{THE STANDARD MODEL}

The Standard Model (SM) of particle physics is a $S U(3)_{C} \times S U(2)_{L} \times U(1)_{Y}$ gauge theory, which describes strong interactions and electroweak interactions among fundamental particles.

There are two types of particle in the Standard Model, fermions and bosons. Fermions, which 
have half-integer spins, are the constituents of matter. There are two types of fermions, leptons and quarks, both of which are described in a three-generation structure. Leptons undergo electromagnetic and weak interactions. In each generation, a charged lepton $\left(e^{-}, \mu^{-}\right.$, or $\left.\tau^{-}\right)$is associated with a neutral neutrino $\left(\nu_{e}, \nu_{\mu}\right.$ or $\left.\nu_{\tau}\right)$. Leptons in the three generations differ by their mass and flavor quantum number. Tab. 1 shows the properties of leptons. Quarks are described in pairs in each generation and have fractional charges $\left(-\frac{1}{3}\right.$ or $\frac{2}{3}$ ). Bound states of quarks form hadrons (baryons or mesons). Properties of quarks are shown in Tab. 2.

Bosons, which have integer spins, play the role of force carriers in the SM interactions. The electromagnetic interaction is mediated by a massless photon $\gamma$, the weak interaction is mediate by massive bosons $W^{ \pm}$and $Z$, while gluons are exchanged in the strong interactions. Property of bosons are summarized in Tab. 3 .

In the Standard Model, neutrinos only participate in weak interactions, and they are described as massless, however, the observation of neutrino oscillation proves that neutrinos have non-zero mass.

\begin{tabular}{ccc}
\hline \hline Flavor & Mass $\left(\mathrm{GeV} / \mathrm{c}^{2}\right)$ & Electric charge \\
\hline electron neutrino $\nu_{e}$ & $<1 \times 10^{-8}$ & 0 \\
electron e & 0.000511 & -1 \\
\hline muon neutrino $\nu_{\mu}$ & $<0.0002$ & 0 \\
muon $\mu$ & 0.106 & -1 \\
\hline tau neutrino $\nu_{\tau}$ & $<0.02$ & 0 \\
$\operatorname{tau} \tau$ & 1.7771 & -1 \\
\hline \hline
\end{tabular}

Table 1: Properties of leptons. 


\begin{tabular}{ccc}
\hline \hline Flavor & Approx. Mass $\left(\mathrm{GeV} / \mathrm{c}^{2}\right)$ & Electric charge \\
\hline up quark (u) & 0.003 & $2 / 3$ \\
down quark (d) & 0.006 & $-1 / 3$ \\
\hline charm quark (c) & 1.3 & $2 / 3$ \\
strange quark (s) & 0.1 & $-1 / 3$ \\
\hline top quark (t) & 175 & $2 / 3$ \\
bottom quark (b) & 4.3 & $-1 / 3$ \\
\hline \hline
\end{tabular}

Table 2: Properties of quarks.

\begin{tabular}{cccc}
\hline \hline name & Mass $\left(\mathrm{GeV} / \mathrm{c}^{2}\right)$ & Electric charge & Force \\
\hline photon $\gamma$ & 0 & 0 & electroweak \\
\hline$W^{-}$ & 80.4 & -1 & electroweak \\
$W^{+}$ & 80.4 & +1 & electroweak \\
$Z^{0}$ & 91.187 & 0 & electroweak \\
\hline gluon g & 0 & 0 & strong \\
\hline \hline
\end{tabular}

Table 3: Properties of bosons.

\subsection{NEUTRINO MIXING AND OSCILLATION}

The history of neutrinos dates back to 1930 when W. Pauli proposed the existence of a new kind of spin-1/2 neutral particle with small mass in order to preserve conservation of energy and momentum in the $\beta$-decay process. Pontecorvo [15] first proposed the concept of neutrino oscillation 
and discussed the oscillation between two flavors of neutrinos [16].

Neutrinos of different flavors can be seen as the linear combination of the mass eigenstates,

$$
\nu_{l}=\sum_{i=1}^{3} U_{l i} \nu_{i}
$$

where $U_{l i}$ is component of the mixing matrix, $\nu_{l}$ denotes a flavor eigenstate $l$, and $\nu_{i}$ denotes a mass eigenstate. Three flavor mixing matrix can be described as the PMNS matrix [17]:

$V_{\mathrm{PMNS}}=\left(\begin{array}{ccc}U_{e 1} & U_{e 2} & U_{e 3} \\ U_{\mu 1} & U_{\mu 2} & U_{\mu 3} \\ U_{\tau 1} & U_{\tau 2} & U_{\tau 3}\end{array}\right)=\left(\begin{array}{ccc}c_{12} c_{13} & s_{12} c_{13} & s_{13} e^{-i \delta} \\ -c_{23} s_{12}-s_{13} s_{23} c_{12} e^{i \delta} & c_{23} c_{12}-s_{13} s_{23} s_{12} e^{i \delta} & s_{23} c_{13} \\ s_{23} s_{12}-s_{13} c_{23} c_{12} e^{i \delta} & -s_{23} c_{12}-s_{13} c_{23} s_{12} e^{i \delta} & c_{23} c_{13}\end{array}\right)$

where $c_{i j}, s_{i j}$ denote $\cos \theta_{i j}, \sin \theta_{i j} . \theta_{i j}$ denotes three mixing angles $\theta_{12}, \theta_{23}$ or $\theta_{13} . \delta$ is the CPviolating phase. In the ultra relativistic limit $m<<E$, the probability of neutrino oscillation from flavor $\alpha$ to flavor $\beta$ can be written as [18]

$$
P(\alpha \rightarrow \beta)=\sum_{i}\left|U_{\beta i}\right|^{2}\left|U_{\alpha i}\right|^{2}+R e \sum_{i \neq j} U_{\beta i} U_{\beta j}^{*} U_{\alpha i} U_{\alpha j}^{*} e^{-i \Delta m_{i j}^{2} L / 2 E}
$$

where $\Delta m_{i j}^{2}=\left|m_{j}^{2}-m_{j}^{2}\right|$ is the difference of mass squared for mass eigenstates $i$ and $j . L$ is the path length from creation to observation, $E$ is the energy of the neutrino, $\alpha, \beta$ are the flavors of neutrinos at the creation and detection point. For three flavor neutrino mixing, there are two differences of mass squared, the solar mass splitting $\Delta m_{21}^{2}=\left|m_{2}^{2}-m_{1}^{2}\right|$ and the atmospheric mass splitting $\Delta m_{31}^{2}=\left|m_{3}^{2}-m_{1}^{2}\right| \cdot m_{2}>m_{1}$ is known from solar and atmospheric neutrino experiments [19], while the sign of $\Delta m_{31}^{2}$ is undetermined. The ordering of neutrino mass states is referred to as the "neutrino mass hierarchy" (MH). The case $m_{3}>m_{1}$ is defined as normal hierarchy (NH), while $m_{3}<m_{1}$ is defined as inverted hierarchy (IH). Tab. 4 shows the best-fit values and $3 \sigma$ allowed ranges of the 3-neutrino oscillation parameters derived from a global fit of the current neutrino oscillation data [2]. Future long-baseline neutrino oscillation experiments, such as Deep Underground Neutrino Experiment (DUNE) [3], are designed to measure unknown parameters, such as $\delta$ and the mass hierarchy. Neutrino and antineutrino cross sections and their shape with energy are needed to measure oscillation parameters. 


\begin{tabular}{|c|c|c|c|}
\hline Parameter & Mass Hierarchy & Best-fit & $3 \sigma$ Parameter range \\
\hline$\Delta m_{21}^{2}$ & & $7.50_{-0.17}^{+0.19}\left(10^{-5} \mathrm{eV}^{2}\right)$ & $7.03 \rightarrow 8.09\left(10^{-5} \mathrm{eV}^{2}\right)$ \\
\hline$\Delta m_{3 l}^{2}$ & Normal & $+2.524_{-0.040}^{+0.039}\left(10^{-3} \mathrm{eV}^{2}\right)$ & $+2.407 \rightarrow+2.643\left(10^{-3} \mathrm{eV}^{2}\right)$ \\
$\Delta m_{3 l}^{2}$ & Inverted & $-2.514_{-0.041}^{+0.038}\left(10^{-3} \mathrm{eV}^{2}\right)$ & $-2.635 \rightarrow-2.399\left(10^{-3} \mathrm{eV}^{2}\right)$ \\
\hline $\sin ^{2} \theta_{12}$ & & $0.306 \pm 0.012$ & $0.271 \rightarrow 0.345$ \\
\hline $\sin ^{2} \theta_{23}$ & Normal & $0.441_{-0.021}^{+0.027}$ & $0.385 \rightarrow 0.635$ \\
$\sin ^{2} \theta_{23}$ & Inverted & $0.587_{-0.024}^{+0.020}$ & $0.393 \rightarrow 0.640$ \\
\hline $\sin ^{2} \theta_{13}$ & Normal & $0.02166 \pm 0.00075$ & $0.01934 \rightarrow 0.02392$ \\
$\sin ^{2} \theta_{13}$ & Inverted & $0.02179 \pm 0.00076$ & $0.01953 \rightarrow 0.02408$ \\
\hline$\delta / \pi$ & Normal & $1.45_{-0.33}^{+0.28}$ & $0 \rightarrow 2.00$ \\
$\delta / \pi$ & Inverted & $1.54_{-0.26}^{+0.22}$ & $0.81 \rightarrow 2.17$ \\
\hline
\end{tabular}

Table 4: The best-fit values and $3 \sigma$ allowed ranges of the 3-neutrino oscillation parameters, derived from a global fit of the current neutrino oscillation data. $\Delta m_{3 l}^{2}=\Delta m_{31}^{2}>0$ for normal hierarchy (NH), $\Delta m_{3 l}^{2}=\Delta m_{32}^{2}<0$ for inverted hierarchy (IH) (Taken from [2]).

The oscillation probability of $\nu_{\mu} \rightarrow \nu_{e}$ through matter, to first order, is given by [20]

$$
\begin{aligned}
P\left(\nu_{\mu} \rightarrow \nu_{e}\right) & =\sin ^{2} \theta_{23} \sin ^{2} 2 \theta_{13} \frac{\sin ^{2}\left(\Delta_{31}-a L\right)}{\left(\Delta_{31}-a L\right)^{2}} \Delta_{31}^{2} \\
& +\sin 2 \theta_{23} \sin 2 \theta_{13} \sin 2 \theta_{12} \frac{\sin \left(\Delta_{31}-a L\right)}{\left(\Delta_{31}-a L\right)} \Delta_{31} \frac{\sin (a L)}{a L} \Delta_{21} \cos \left(\Delta_{31}+\delta\right) \\
& +\cos ^{2} \theta_{23} \sin ^{2} 2 \theta_{12} \frac{\sin ^{2}(a L)}{(a L)^{2}}
\end{aligned}
$$

where $\Delta_{i j}=\Delta m_{i j}^{2} L / 4 E_{\nu}, a=G_{F} N_{e} / \sqrt{2}, G_{F}$ is the Fermi constant, $N_{e}$ is the density of electrons in the Earth's crust, $\mathrm{L}$ is the baseline in $\mathrm{km}$, and $E_{\nu}$ is the neutrino energy in $\mathrm{GeV}$. For the probability of $\overline{\nu_{\mu}} \rightarrow \overline{\nu_{e}}$, both $\delta$ and $a$ (matter effect) change sign in Eq. 1.4, which is written as 


$$
\begin{aligned}
P\left(\overline{\nu_{\mu}} \rightarrow \overline{\nu_{e}}\right) & =\sin ^{2} \theta_{23} \sin ^{2} 2 \theta_{13} \frac{\sin ^{2}\left(\Delta_{31}+a L\right)}{\left(\Delta_{31}+a L\right)^{2}} \Delta_{31}^{2} \\
& +\sin 2 \theta_{23} \sin 2 \theta_{13} \sin 2 \theta_{12} \frac{\sin \left(\Delta_{31}+a L\right)}{\left(\Delta_{31}+a L\right)} \Delta_{31} \frac{\sin (a L)}{a L} \Delta_{21} \cos \left(\Delta_{31}-\delta\right) \\
& +\cos ^{2} \theta_{23} \sin ^{2} 2 \theta_{12} \frac{\sin ^{2}(a L)}{(a L)^{2}}
\end{aligned}
$$

If the $\mathrm{CP}$ violating phase term, $\delta$, differs from zero or $\pi$, it is relevant to the matter-antimatter asymmetry in the universe. The $\mathrm{CP}$ asymmetry is defined as

$$
A_{c p}=\frac{P\left(\nu_{\mu} \rightarrow \nu_{e}\right)-P\left(\overline{\nu_{\mu}} \rightarrow \overline{\nu_{e}}\right)}{P\left(\nu_{\mu} \rightarrow \nu_{e}\right)+P\left(\overline{\nu_{\mu}} \rightarrow \overline{\nu_{e}}\right)}
$$

To leading order in $\Delta m_{21}^{2}$, it can be written as

$$
A_{c p} \simeq \frac{\cos \theta_{23} \sin 2 \theta_{12} \sin \delta}{\sin \theta_{23} \sin \theta_{13}}\left(\frac{\Delta m_{21}^{2} L}{4 E_{\nu}}\right)+\text { matter effects }
$$

Fig. 1 (left) shows the $\nu_{e}$ appearance probability as a function of neutrino energy for different $\delta$ values $\left(\delta=-\frac{\pi}{2}, 0,+\frac{\pi}{2}\right)$ for normal hierarchy. The probability changes largely with different values of $\delta$. Fig. 1 (right) shows the reconstructed neutrino energy distribution of selected events for neutrinos with normal hierarchy for $\delta=0$. In order to measure the observed shape of $\nu_{\mu} \rightarrow \nu_{e}$ and $\overline{\nu_{\mu}} \rightarrow \overline{\nu_{e}}$ below $10 \mathrm{GeV}$, knowing neutrino and antineutrino interaction cross sections and fluxes well in this energy region is essential.

With a baseline of $1300 \mathrm{~km}$, DUNE will be capable of measuring the sign of $\Delta_{31}$ through the matter effect term that also causes a CP asymmetry. The matter effect and $\delta$ effect on $A_{c p}$ need to be disentangled in the electron neutrino appearance probability. 

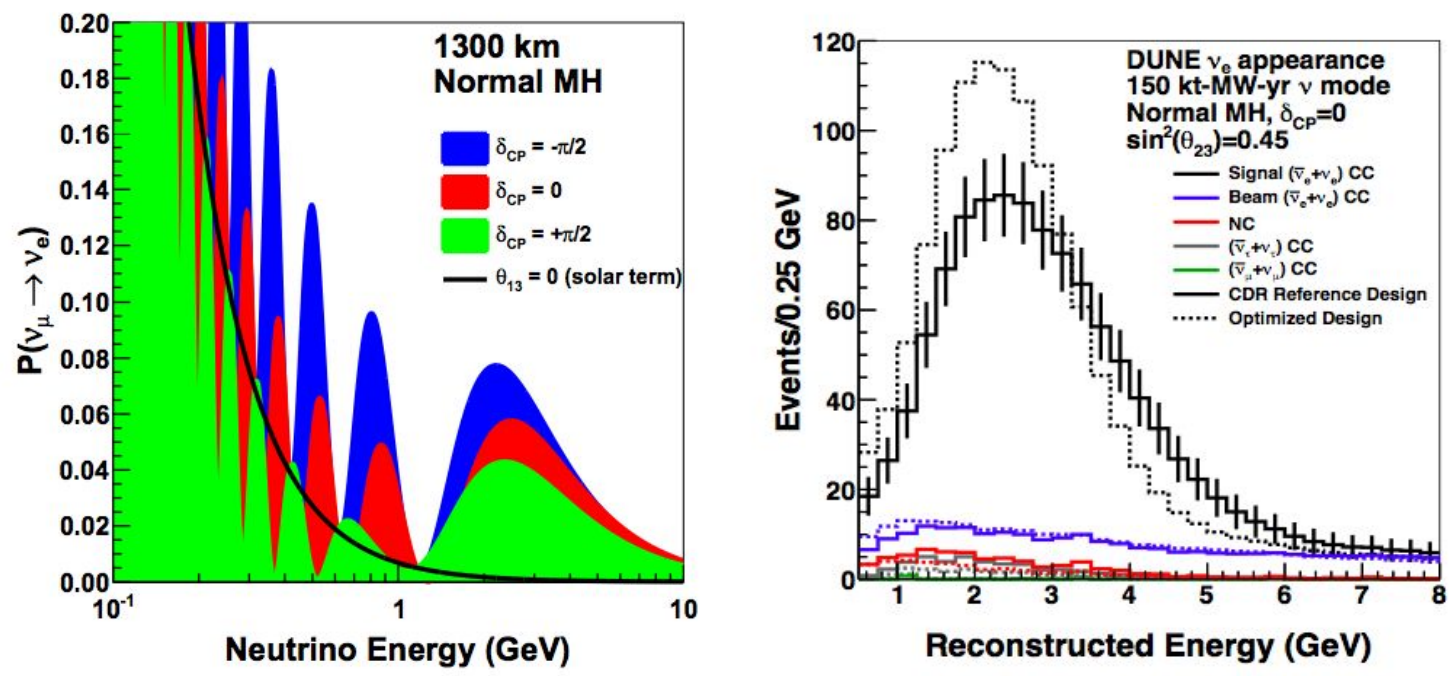

Figure 1: Left shows the $\nu_{e}$ appearance probability as a function of neutrino energy at $1300 \mathrm{~km}$ for different $\delta$ values for neutrinos for normal hierarchy. Right shows simulated energy distribution of DUNE events assuming normal hierarchy, $\delta_{C P}=0$ and a $150 \mathrm{kt} \cdot \mathrm{MW}$. year exposure in the neutrino-beam mode (Both taken from [3]). 


\subsection{NEUTRINO INTERACTIONS}

As described by the Standard Model, neutrino interactions are weak interactions via leptonic charged current $j_{W}^{\mu}$ and neutral current $j_{W}^{\mu}$, which are written as,

$$
\begin{gathered}
j_{W}^{\mu}=2 \sum_{\alpha=e, \mu, \tau} \overline{\nu_{L, \alpha}} \gamma^{\mu} l_{\alpha L}, \\
j_{Z}^{\mu}=2 \sum_{\alpha=e, \mu, \tau} g_{L}^{\nu} \overline{\nu_{\alpha L}} \gamma^{\mu} \nu_{\alpha L}+g_{L}^{f} \overline{l_{\alpha L}} \gamma^{\mu} l_{\alpha L}+g_{R}^{f} \overline{l_{\alpha R}} \gamma^{\mu} l_{\alpha R} .
\end{gathered}
$$

The Lagrangian describing neutrino interactions is written as,

$$
\begin{gathered}
\mathscr{L}_{C C}=-\frac{g}{2 \sqrt{2}}\left(j_{W}^{\mu} W_{\mu}+j_{W}^{\mu, \dagger} W_{\mu}^{\dagger}\right), \\
\mathscr{L}_{N C}=-\frac{g}{2 \cos \theta_{W}} j_{Z}^{\mu} Z_{\mu} .
\end{gathered}
$$

\subsubsection{Kinematics}

Fig. 2 shows the tree level Feynman diagram of a muon neutrino-nucleon charged-current scattering. A muon neutrino with four momentum $k_{1}$ scatters off a nucleon with momentum $\mathrm{P}$, the final state includes a muon with four momentum $k_{2}$ and a recoil hadronic system. The energy transferred to the hadronic system is defined as

$$
\nu=\frac{P \cdot q}{M} .
$$

The inelasticity is defined as

$$
y=\frac{P \cdot q}{P \cdot k_{1}} .
$$

The momentum transfer to the nucleon, negative squared four-momentum of the boson, is defined as

$$
Q^{2}=-q^{2}=-\left(k_{1}-k_{2}\right)^{2} .
$$

The Bjorken scaling variable is defined as

$$
x=\frac{Q^{2}}{2 P \cdot q} .
$$




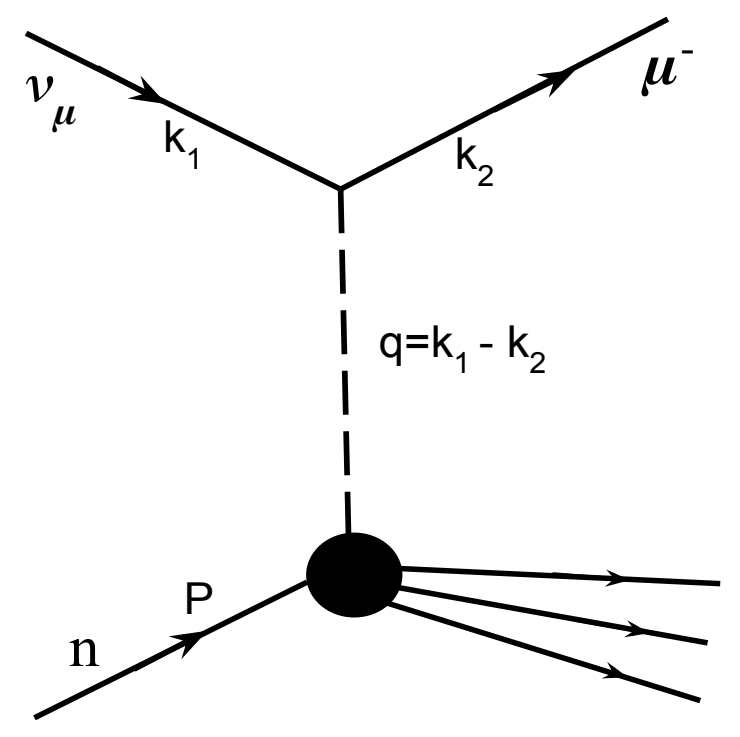

Figure 2: Feynman diagram of $\nu_{\mu} N \mathrm{CC}$ interaction.

The invariant mass of the boson-nucleon system is defined as

$$
W^{2}=(P+q)^{2}
$$

In lab frame, the nucleon is at rest and its four-momentum is $P=(M, 0,0,0)$, the kinematic variables can be written as

$$
\begin{gathered}
\nu=E_{\nu}-E_{\mu}=E_{h a d}, \\
y=\frac{E_{h a d}}{E_{\mu}}, \\
Q^{2}=2 E_{\nu} E_{\mu}\left(1-\cos \theta_{\mu}\right), \\
x=\frac{Q^{2}}{2 M \nu}, \\
W^{2}=M^{2}-Q^{2}+2 M \nu .
\end{gathered}
$$




\subsubsection{Deep-inelastic scattering (DIS)}

In deep inelastic scattering, the neutrino scatters off a quark in the nucleon via the exchange of a virtual $\mathrm{W}$ or $\mathrm{Z}$ boson producing a lepton and a hadronic system in the final state. The Feynman diagram of deep-inelastic scattering is shown in Figure 3. The differential cross section for neutrino

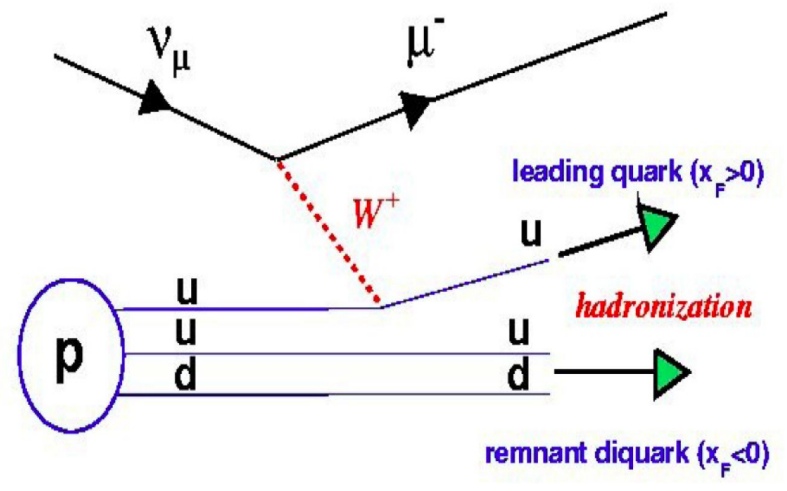

Figure 3: An example of charged-current deep-inelastic scattering. A muon neutrino scatters off a proton, producing a muon and a hadron shower.

(or antineutrino) inelastic interaction can be written as [21]

$\frac{d^{2} \sigma^{\nu(\bar{\nu}) N}}{d x d y}=\frac{G_{F}^{2} M E_{\nu}}{\pi\left(1+Q^{2} / M_{W}^{2}\right)^{2}}\left[\frac{y^{2}}{2} 2 x F_{1}^{\nu(\bar{\nu}) N}\left(x, Q^{2}\right)+\left(1-y-\frac{M x y}{2 E_{\nu}}\right) F_{2}^{\nu(\bar{\nu}) N}\left(x, Q^{2}\right) \pm y\left(1-\frac{y}{2}\right) x F_{3}^{\nu(\bar{\nu}) N}\right]$

where $F_{1}, F_{2}$ and $F_{3}$ are structure functions that describe the quark structure of the nucleon. The plus (minus) sign in front of $x F_{3}$ is for neutrino (antineutrino). The ratio of the longitudinal to transverse virtual boson absorption cross section is often defined as,

$$
R_{L}\left(x, Q^{2}\right)=\frac{\sigma_{L}}{\sigma_{T}}=\frac{F_{2}\left(x, Q^{2}\right)\left(1+4 M^{2} x^{2} / Q^{2}\right)-2 x F_{1}\left(x, Q^{2}\right)}{2 x F_{1}\left(x, Q^{2}\right)},
$$

The quark parton model (QPM) [22] describes the structure functions in terms of parton distribution functions (PDFs). In QPM, a nucleon is described as a system with 3 valence quarks and sea quarks. For quark $q$ and anti-quark $\bar{q}$, there PDFs are written as

$$
q(x)=q_{v}(x)+q_{s}(x)
$$


and

$$
\bar{q}(x)=\bar{q}_{s}(x)=q_{s}(x)
$$

the subscript $v$ stands for valence quark and the subscript $s$ stands for sea quark.

According to Callan-Gross relation [23], for scattering off spin- $\frac{1}{2}$ constituents,

$$
2 x F_{1}=F_{2} \text {. }
$$

For neutrino interactions with proton (constituted by spin- $\frac{1}{2}$ partons),

$$
2 x F_{1}^{\nu p}(x)=F_{2}^{\nu p}(x)=2 x(d(x)+\bar{u}(x)+s(x)+\bar{c}(x)),
$$

and

$$
x F_{3}^{\nu p}(x)=2 x(d(x)-\bar{u}(x)+s(x)-\bar{c}(x)),
$$

where $u(x), d(x), s(x)$ and $c(x)$ stands for the parton distribution functions for up, down, strange and charm quarks respectively, which describe the probability of a quark carrying fraction $x$ of the momentum of a nucleon. Similarly, structure functions for neutrino neutron interaction can be written as

$$
2 x F_{1}^{\nu n}(x)=F_{2}^{\nu n}(x)=2 x(u(x)+\bar{d}(x)+s(x)+\bar{c}(x))
$$

and

$$
x F_{3}^{\nu n}(x)=2 x(u(x)-\bar{d}(x)+s(x)-\bar{c}(x)) .
$$

Combining these structure functions for scattering from an isoscalar target (defined as equal number of neutrons and protons) are given as

$$
F_{2}^{\nu N}(x)=x(u(x)+d(x)+2 s(x)+\bar{u}(x)+\bar{d}(x)+2 \bar{c}(x)),
$$

and

$$
x F_{3}^{\nu N}(x)=x(u(x)+d(x)+2 s(x)-\bar{u}(x)-\bar{d}(x)-2 \bar{c}(x))
$$

assuming that $s(x)=\bar{s}(x)$, and $c(x)=\bar{c}(x)$.

For antineutrinos, assuming there is no quark mixing, the isoscalar structure functions are

$$
F_{2}^{\bar{\nu} N}(x)=F_{2}^{\nu N}(x)
$$


and

$$
x F_{3}^{\bar{\nu} N}(x)=x F_{3}^{\nu N}(x)-4 x(s(x)-c(x)) .
$$

If considering quark mixing, the structure functions $F_{2}$ are written as

$F_{2}^{\nu}(x)=V_{c d}^{2}\left(\frac{u_{v}+d_{v}}{2}\right)+V_{c d}^{2}\left(\frac{u_{s}+d_{s}}{2}\right)+s_{s} V_{c s}^{2}+V_{u d}^{2}\left(\frac{u_{v}+d_{v}}{2}\right)+V_{u d}^{2}\left(u_{s}+d_{s}\right)+V_{u s}^{2}\left(\frac{u_{s}+d_{s}}{2}\right)+s_{s} V_{v s}^{2}$

and

$F_{2}^{\bar{\nu}}(x)=V_{c d}^{2}\left(\frac{u_{s}+d_{s}}{2}\right)+s_{s} V_{c s}^{2}+V_{u d}^{2}\left(\frac{u_{v}+d_{v}}{2}\right)+V_{u d}^{2}\left(u_{s}+d_{s}\right)+V_{u s}^{2}\left(\frac{u_{s}+d_{s}}{2}\right)+s_{s} V_{v s}^{2}+V_{u s}^{2}\left(\frac{u_{v}+d_{v}}{2}\right)$.

$V_{c d}, V_{c s}, V_{u s}$ and $V_{u d}$ are quark mixing angles from the CKM quark mixing matrix [24]. Eqs. 1.34 and 1.35 give a small difference between neutrino and antineutrino's structure functions $F_{2}$, which will be used in Sec. 6.1.

Quantum Chromo Dynamics (QCD) introduces additional quantum number, color. It describes the interactions between quarks and gluons within SU(3) group of color, which is referred to as the strong interaction. One property of QCD is the confinement, it explains the lack of observation of free quarks. Quarks and gluons carry color number, however, they can only be observed in hadrons which are colorless. Gluons interact with themselves since they also carry the color charges. A large amount of energy, which increases linear with distance, is required to break up hadrons. QCD predicts that with $Q^{2}$ increasing, structure functions increase at small $x$ and decrease at larger $x$.

\subsubsection{Resonance production (RES)}

Neutrinos can also inelastically scatter producing a nucleon excited state $\left(\Delta, N^{*}\right)$. The Feynman diagram of resonance production is shown in Figure 4. Such baryonic resonances quickly decay, most often to a nucleon and single pion final state. In this section we briefly show the calculation of cross section for a single pion production and the dynamics of this process in the FKR [25] model following Ref. [26].

For the process

$$
\nu+N \rightarrow l+N^{*},
$$




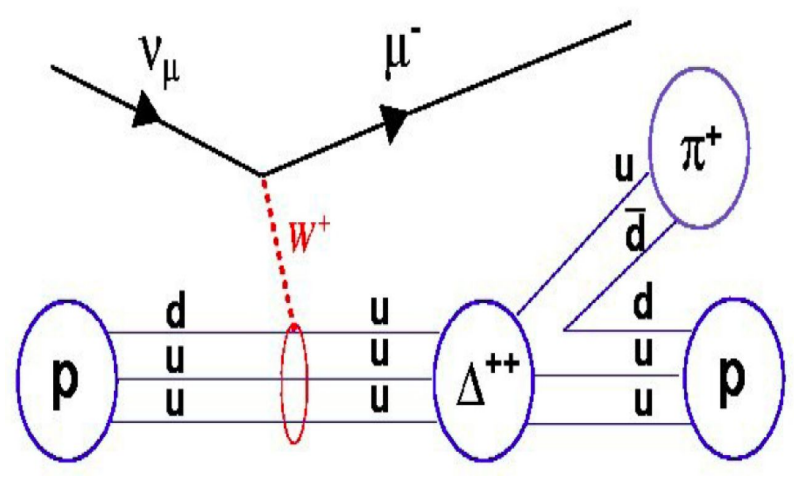

Figure 4: An example of charged-current single pion production. A muon neutrino scattering off a proton, producing a muon, a pion and a proton in the final state.

the production matrix is given by

$$
T\left(\nu N \rightarrow l N^{*}\right)=\frac{G}{\sqrt{2}}\left[\bar{u}_{l} \gamma^{\beta}\left(1-\gamma_{5}\right) u_{\nu}\right]\left\langle N^{*}\left|J_{\beta}^{+}(0)\right| N\right\rangle .
$$

The hadronic current operator contains a vector and an axial vector part,

$$
J_{\beta}^{+}=V_{\beta}-A_{\beta}=2 M F_{\beta}=2 M\left(F_{\beta}^{V}-F_{\beta}^{A}\right),
$$

where $M$ is the resonance mass, $F_{\beta}^{V}$ and $F_{\beta}^{A}$ are charged current operator corresponding to vector and axial vector part, respectively. Define three standard polarization vectors $e_{L}^{\mu}, e_{R}^{\mu}$ and $e_{0}^{\mu}$, which corresponding to left-handed, right-handed and scalar polarization, the leptonic current can be rewritten as

$$
\bar{u}_{l} \gamma^{u}\left(1-\gamma_{5}\right) u_{\nu}=-2 \sqrt{2} E \sqrt{\frac{-q^{2}}{Q^{2}}}\left\{u \cdot e_{L}^{\mu}-v \cdot e_{R}^{\mu}+\sqrt{2 u v} \cdot e_{s}^{\mu}\right\}
$$

in which $E$ is the energy of incident neutrino, $Q$ is the modulus of 3-momentum transfer in the lab frame and $q^{2}=\nu^{2}-Q^{2}$. The 4-momentum of $e_{s}^{\mu}$ is defined as

$$
e_{s}^{\mu}=\frac{1}{-q^{2}}\left(Q^{*}, 0,0, \nu^{*}\right) .
$$


Then, the full matrix element is written as

$$
T\left(\nu N \rightarrow l N^{*}\right)=-4 G M E\left\{\sqrt{\frac{-q^{2}}{Q^{2}}}\left\langle N^{*}\left|u F_{-}-v F_{+}\right| N\right\rangle+\frac{m_{N}}{M} \sqrt{2 u v}\left\langle N^{*}\left|F_{0}\right| N\right\rangle\right\},
$$

in which $m_{N}$ is the nucleon mass, with

$$
\begin{gathered}
F_{+}=e_{R}^{\mu} F_{\mu}=-\frac{1}{\sqrt{2}}\left(F_{x}+i F_{y}\right), \\
F_{-}=e_{L}^{\mu} F_{\mu}=\frac{1}{\sqrt{2}}\left(F_{x}-i F_{y}\right),
\end{gathered}
$$

and

$$
F_{0}=\sqrt{\frac{-q^{2}}{Q^{* 2}}} e_{s}^{\mu} F_{\mu}=F_{t}+\frac{\nu^{*}}{Q^{*}} F_{z},
$$

where $F_{x}, F_{y}, F_{z}$ and $F_{t}$ are components corresponding to the $x, y, z$ and $t$ direction of 4momentum. The production differential cross section of a single resonance with mass $M$ and negligible width is written as

$$
\frac{d \sigma}{d q^{2} d \nu}=\frac{1}{32 \pi m_{N} E^{2}} \cdot \frac{1}{2} \cdot \sum_{\text {spins }}\left|T\left(\nu N \rightarrow l N^{*}\right)\right|^{2} \delta\left(W^{2}-M^{2}\right) .
$$

Adding up three terms of $T$ incoherently gives

$$
\frac{d \sigma}{d q^{2} d \nu}=\frac{G^{2}}{4 \pi^{2}}\left(\frac{-q^{2}}{Q^{2}}\right) \kappa\left\{u^{2} \sigma_{L}+v^{2} \sigma_{R}+2 u v \sigma_{s}\right\},
$$

where $\sigma_{L}, \sigma_{R}$ and $\sigma_{s}$ stand for the cross sections for the absorption of an intermediate vector boson with positive, negative or zero helicity, which has $\delta(W-M)$ term in them. The conventional kinematical factor is

$$
\kappa=\nu+\frac{q^{2}}{2 m_{N}}=\frac{M^{2}-m_{N}^{2}}{2 m_{N}} .
$$

For incident antineutrinos, the matrix element is given by

$$
T\left(\bar{\nu} N \rightarrow \bar{l} N^{*}\right)=-4 G M E\left\{\sqrt{\frac{-q^{2}}{Q^{2}}}\left\langle N^{*}\left|u \bar{F}_{+}-v \bar{F}_{-}\right| N\right\rangle-\frac{m_{N}}{M} \sqrt{2 u v}\left\langle N^{*}\left|\bar{F}_{0}\right| N\right\rangle\right\} .
$$

The antineutrino differential cross section is given by

$$
\frac{d \bar{\sigma}}{d q^{2} d \nu}=\frac{G^{2}}{4 \pi^{2}}\left(\frac{-q^{2}}{Q^{2}}\right) \kappa\left\{u^{2} \bar{\sigma}_{R}+v^{2} \bar{\sigma}_{L}+2 u v \bar{\sigma}_{s}\right\},
$$


in which $\bar{\sigma}_{L}, \bar{\sigma}_{R}$ and $\bar{\sigma}_{s}$ stand for the antineutrino cross sections for the absorption of an intermediate vector boson with positive, negative or zero helicity. For resonances of finite width, the $\delta$-function is replaced by a Breit-Wigner factor

$$
\delta(W-M) \rightarrow \frac{1}{2 \pi} \cdot \frac{\Gamma}{(W-M)^{2}+\Gamma^{2} / 4},
$$

in which, $\Gamma$ is the resonance width. Then, the total cross sections can be obtained by integrating Eq. 1.46 and 1.49 within $W$ and $q^{2}$ bounds.

The FKR model, proposed by Feynman, Kislinger and Ravndal, is a harmonic oscillator quark model. It is adopted by [26] to compute the production and decay amplitudes. In FKR model, the hamiltonian of a four-dimensional harmonic oscillator is written as

$$
H=3\left(p_{a}^{2}+p_{b}^{2}+p_{c}^{2}\right)+\frac{1}{36} \Omega^{2}\left[\left(u_{a}-u_{b}\right)^{2}+\left(u_{b}-u_{c}\right)^{2}+\left(u_{c}-u_{a}\right)^{2}\right]+\text { const }
$$

where $p_{a}$ is the four-momentum operator of quark a, and $u_{a}$ is its conjugate position, which means $p_{a}=i \frac{\partial}{\partial u_{a}}$. The electromagnetic and weak interaction terms can be simplified as

$$
e^{\mu} j_{\mu}^{V, A}=2 W\left(e_{t} F_{t}^{V, A}+e_{z} F_{z}^{V, A}-e_{+} F_{-}^{V, A}-e_{-} F_{+}^{V, A}\right)
$$

in which $e^{\mu}$, is a wave of polarization vector, $j_{\mu}^{V, A}$ is vector or axial-vector current, $F_{ \pm}^{V, A}$ and $F_{0}^{V, A}$ are charged current which include a dipole factor term

$$
G^{V, A}\left(q^{2}\right)=\left(1-\frac{q^{2}}{4 m_{N}^{2}}\right)^{1 / 2-n}\left(\frac{1}{1-q^{2} / m_{V, A}^{2}}\right)^{2} .
$$




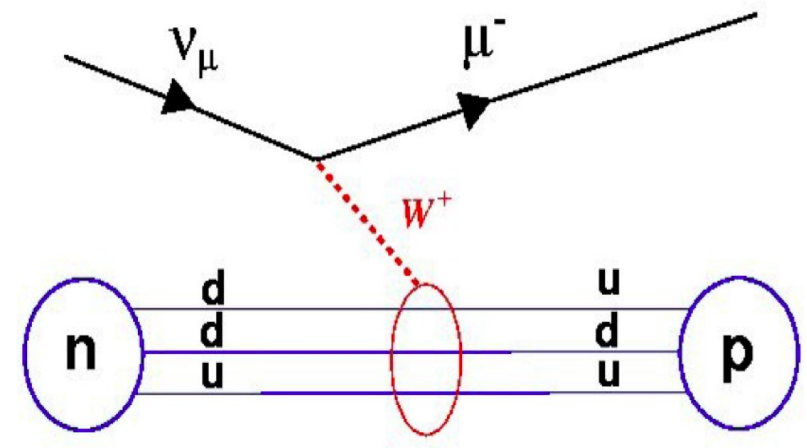

Figure 5: An example of charged-current quasi-elastic scattering. Incoming muon neutrino scatters off a neutron and produces a muon and a proton in the final state.

\subsubsection{Quasi-elastic scattering(QEL)}

The Feynman diagram for quasi-elastic scattering is shown in Fig. 5. Neutrino (or antineutrino) quasi-elastic scattering refers to the processes, $\nu_{\mu}+n \rightarrow \mu^{-}+p$ (or $\left.\overline{\nu_{\mu}}+p \rightarrow \mu^{+}+n\right)$. A charged lepton and single nucleon are ejected in the elastic interaction of a neutrino (or antineutrino) with a nucleon in the target material. The nucleon changes, but does not break up. The hadronic current describing the process of neutrino scattering off a nucleon

$$
\nu\left(k_{1}\right)+n\left(p_{1}\right) \rightarrow l^{-}\left(k_{2}\right)+p\left(p_{2}\right)
$$

can be written as

$$
\left\langle p\left(p_{2}\right)\left|J_{\lambda}^{+}\right| n\left(p_{1}\right)\right\rangle=\cos \theta_{c} \bar{u}\left(p_{2}\right) \Gamma_{\lambda} u\left(p_{1}\right)
$$

in which $\nu$ is the incoming neutrino with momentum $k_{1}, n$ is the nucleon with momentum $p_{1}$, while the final state includes a lepton $l^{-}$with momentum $k_{2}$ and a nucleon $p$ with momentum $p_{2} . J_{\lambda}^{+}$ is the current which increases the charge of initial state nucleon the neutrino interacts with. $\theta_{c}$ is Cabibbo angle and $\Gamma_{\lambda}$ is a function of different form factors which are described below. Similarly, for antineutrino case, the current may be written as

$$
\left\langle n\left(p_{2}\right)\left|J_{\lambda}^{-}\right| p\left(p_{1}\right)>=\cos \theta_{c} \bar{u}\left(p_{2}\right) \widetilde{\Gamma_{\lambda}} u\left(p_{1}\right)=<p\left(p_{1}\right)\left|J_{\lambda}^{+}\right| n\left(p_{2}\right)\right\rangle^{*}
$$


in which

$$
\widetilde{\Gamma_{\lambda}}\left(p_{1}, p_{2}\right)=\gamma_{0} \Gamma_{\lambda}^{+}\left(p_{2}, p_{1}\right) \gamma_{0}
$$

and $*$ denotes its conjugated term. In [27], the differential cross section is derived as

$$
\frac{d \sigma}{d\left|q^{2}\right|}\left(\begin{array}{l}
\nu n \rightarrow l^{-} p \\
\bar{\nu} p \rightarrow l^{+} n
\end{array}\right)=\frac{M^{2} G^{2} \cos ^{2} \theta_{c}}{8 \pi E_{\nu}^{2}}\left[A\left(q^{2}\right) \mp B\left(q^{2}\right) \frac{(s-u)}{M^{2}}+\frac{C\left(q^{2}\right)(s-u)^{2}}{M^{4}}\right],
$$

where $s$ and $u$ are Mandelstam variables and

$$
s-u=4 M E_{\nu}+q^{2}-m^{2} .
$$

$A, B$ and $C$ are functions of form factors, and given explicitly below

$$
\begin{gathered}
A\left(q^{2}\right)=\frac{\left(m^{2}-q^{2}\right)}{4 M^{2}}\left[\left(4-\frac{q^{2}}{M^{2}}\right)\left|F_{A}\right|^{2}-\left(4+\frac{q^{2}}{M^{2}}\right)\left|F_{V}^{1}\right|^{2}-\frac{q^{2}}{M^{2}}\left|\xi F_{V}^{2}\right|^{2}\left(1+\frac{q^{2}}{4 M^{2}}\right)\right. \\
-\frac{4 q^{2} R e F_{V}^{1} \xi F_{V}^{2}}{M^{2}}+\frac{q^{2}}{M^{2}}\left(4-\frac{q^{2}}{M^{2}}\right)\left|F_{A}^{3}\right|^{2}-\frac{m^{2}}{M^{2}}\left(\left|F_{V}^{1}+\xi F_{V}^{2}\right|^{2}+\right. \\
\left.\left.\left|F_{A}+2 F_{P}\right|^{2}+\left(\frac{q^{2}}{M^{2}}-4\right)\left(\left|F_{V}^{3}\right|^{2}+\left|F_{P}\right|^{2}\right)\right)\right], \\
B=-\frac{q^{2}}{M^{2}} \operatorname{Re}_{A}^{*}\left(F_{V}^{1}+\xi F_{V}^{2}\right)-\frac{m^{2}}{M^{2}} \operatorname{Re}\left[\left(F_{V}^{1}+\frac{q^{2}}{4 M^{2}} \xi F_{V}^{2}\right)^{*} F_{V}^{3}-\left(F_{A}+\frac{q^{2} F_{p}}{2 M^{2}}\right)^{*} F_{A}^{3}\right],
\end{gathered}
$$

and

$$
C=\frac{1}{4}\left(\left|F_{A}\right|^{2}+\left|F_{V}^{1}\right|^{2}-\frac{q^{2}}{M^{2}}\left|\frac{\xi F_{V}^{2}}{2}\right|^{2}-\frac{q^{2}}{M^{2}}\left|F_{A}^{3}\right|^{2}\right),
$$

where the axial form factor

$$
F_{A}\left(q^{2}\right)=\frac{F_{A}(0)}{\left(1-\frac{q^{2}}{M_{A}^{2}}\right)^{2}}
$$

describes the axial structure of a nucleon, in which $M_{A}$ is referred to as the axial mass. The pseudo-scalar form factor is given by

$$
F_{p}\left(q^{2}\right)=\frac{2 M^{2} F_{A}\left(q^{2}\right)}{M_{\pi}^{2}-q^{2}}
$$

in which $M_{\pi}$ is the charged pion mass and $M$ is the mass of the nucleon. 


\subsection{STATUS OF CHARGED-CURRENT INCLUSIVE CROSS SECTION MEASUREMENT}

The total charged-current cross section is the sum of these three components (DIS, RES and QEL), as well as other small components, such as coherence $(\mathrm{COH})$, which we will not discuss in detail. Figure 6 is an illustration of how each component dominates in different neutrino energy regions. The inclusive cross section is dominated by DIS above $10 \mathrm{GeV}$. QEL, RES and DIS all contribute below $10 \mathrm{GeV}$.
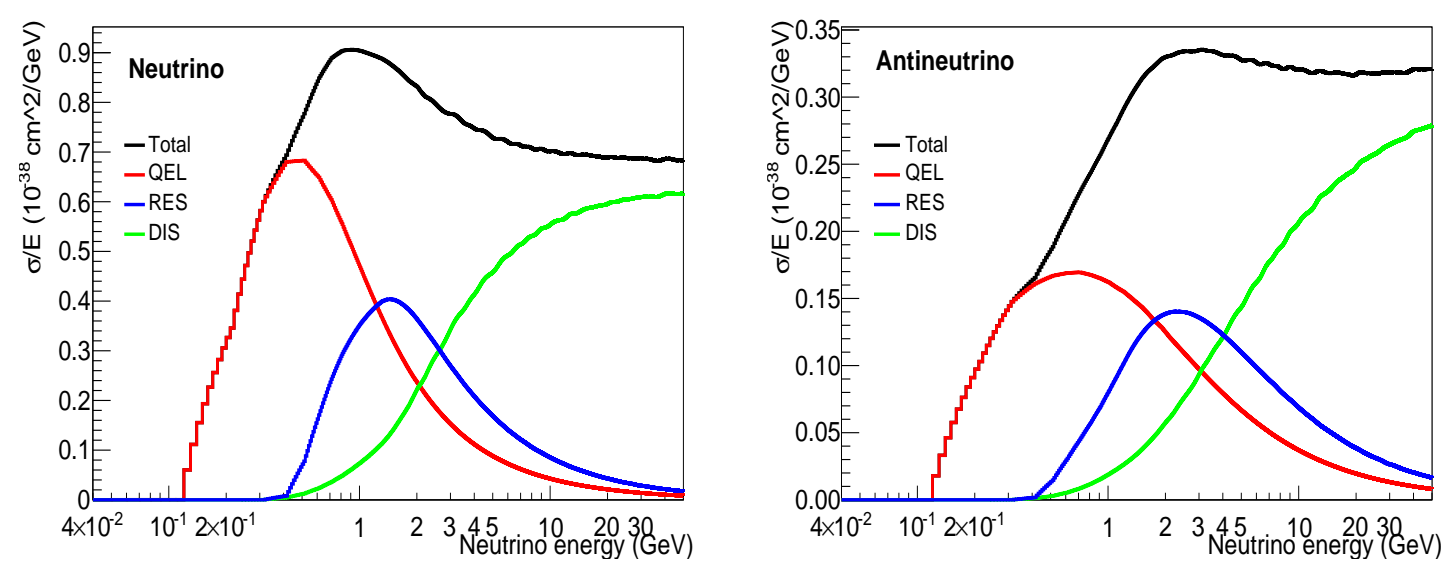

Figure 6: Neutrino (left) and antineutrino (right) total charged-current cross sections and the components of QEL, DIS and RES. Those plots are made with GENIE 2.8.4, which is the model used to simulate events in this analysis.

Many experiments have measured the charged-current inclusive cross sections for neutrino and antineutrino scattering off nucleons [24], as shown in Figure 7. At high energy (above 20 $\mathrm{GeV}$ ), the inclusive cross sections are precisely measured to be almost linear with neutrino energy (dominated by DIS). Below $10 \mathrm{GeV}$, they are not well measured, especially for antineutrinos, due to the limited knowledge and data. 


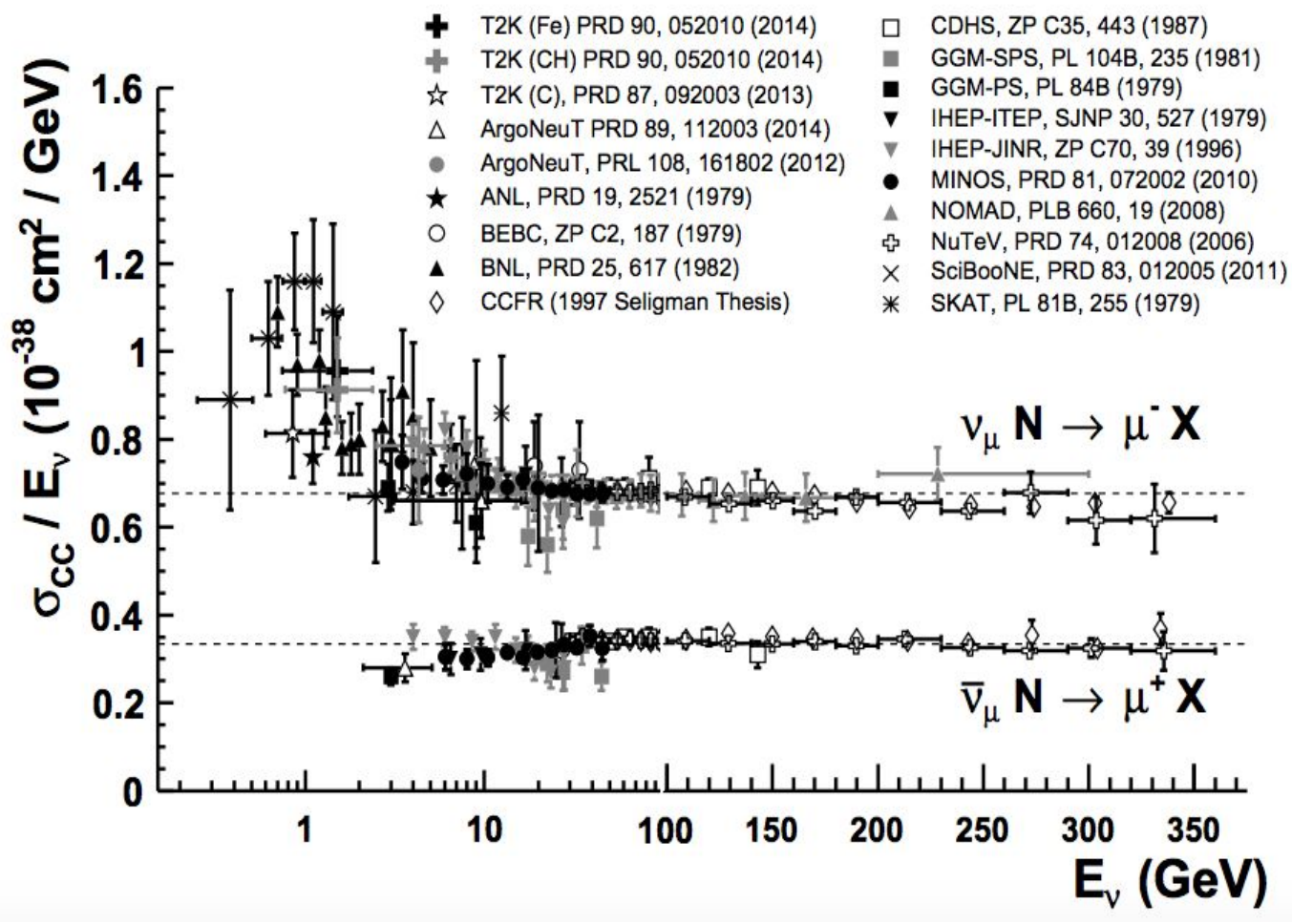

Figure 7: Measurements of $\nu_{\mu}$ and $\overline{\nu_{\mu}} \mathrm{CC}$ inclusive scattering cross sections divided by neutrino energy as a function of neutrino energy (Taken from [4]). 
Neutrino cross sections were firstly measured by bubble chamber experiments in 1970s and 1980s, which includes ANL [28] [29], BNL [30][31], Gargamelle [32] [33] and SKAT [34]. Then followed more precise experiments on different nuclei, ITEP [35] on iron and JINR [36] on aluminum. More recently, NOMAD [37] measured neutrino cross sections on carbon from 2.5 - 40 $\mathrm{GeV}$ with $4 \%$ uncertainty, which is smaller than previous measurements. Also, MINOS [38] reported isoscalar corrected neutrino cross section on iron with similar size of uncertainty in 3-50 GeV energy range. Recently, SciBooNE [39] reports neutrino cross section in the energy range of 0.38-2.47 GeV. ArgoNeuT [40] reported neutrino cross section at $4.3 \mathrm{GeV}$ with an uncertainty of $16 \%$. T2K measured the neutrino cross section at $0.85 \mathrm{GeV}$ with an uncertainty of $12 \%$ [41] in the energy range 1.0-3.3 GeV with uncertainty $13 \%$ - 19\% [42]. Our measurement of neutrino cross section overlaps in the energy range $2-22 \mathrm{GeV}$ with comparable precision as previous measurements.

Antineutrino cross sections at low neutrino energy are less well measured compared with neutrino cross sections. Most measurements were performed in the 1970s and 1980s, such as measurements by BNL [43], FNAL [44], Gargamelle [32] using bubble chambers, and ITEP [45] [35] on iron. Then followed the measurement of JINR [36] on aluminum in 1990s. The most recent measurements are MINOS [38] on iron above $5 \mathrm{GeV}$ and ArgoNeuT [46] at 3.6 GeV. Our measurement is the most precise to date below $6 \mathrm{GeV}$.

The ratio of antineutrino to neutrino cross section, $r$, is poorly measured as well. Fig. 8 shows all previous measurements. The first $r$ measurement was performed by the bubble chamber experiment Gargamelle [32] in 1-10 GeV, with an uncertainty of 18\% - 90\%. Then, ITEP [35] measured $r$ in $3-30 \mathrm{GeV}$ on iron with an uncertainty of $8 \%-20 \%$. The third and the latest measurement was by MINOS, in the energy region of 6-50 GeV, which greatly improved the precision to $7.2 \%$ $2.2 \%$.

In this thesis, we report the measurement of neutrino and antineutrino inclusive cross sections below $22 \mathrm{GeV}$ with uncertainty of $4.3 \%-10.1 \%$ for neutrino and $6.1 \%-10.5 \%$ for antineutrino, as well as the antineutrino to neutrino ratio with an uncertainty of 5.0\%-7.5\%. The ratio benefits from the cancellation of systematic uncertainties between neutrino and antineutrino. The measurement of $r$ also provides information about neutrino spectra needed for determining CP asymmetry in oscillation experiments. 


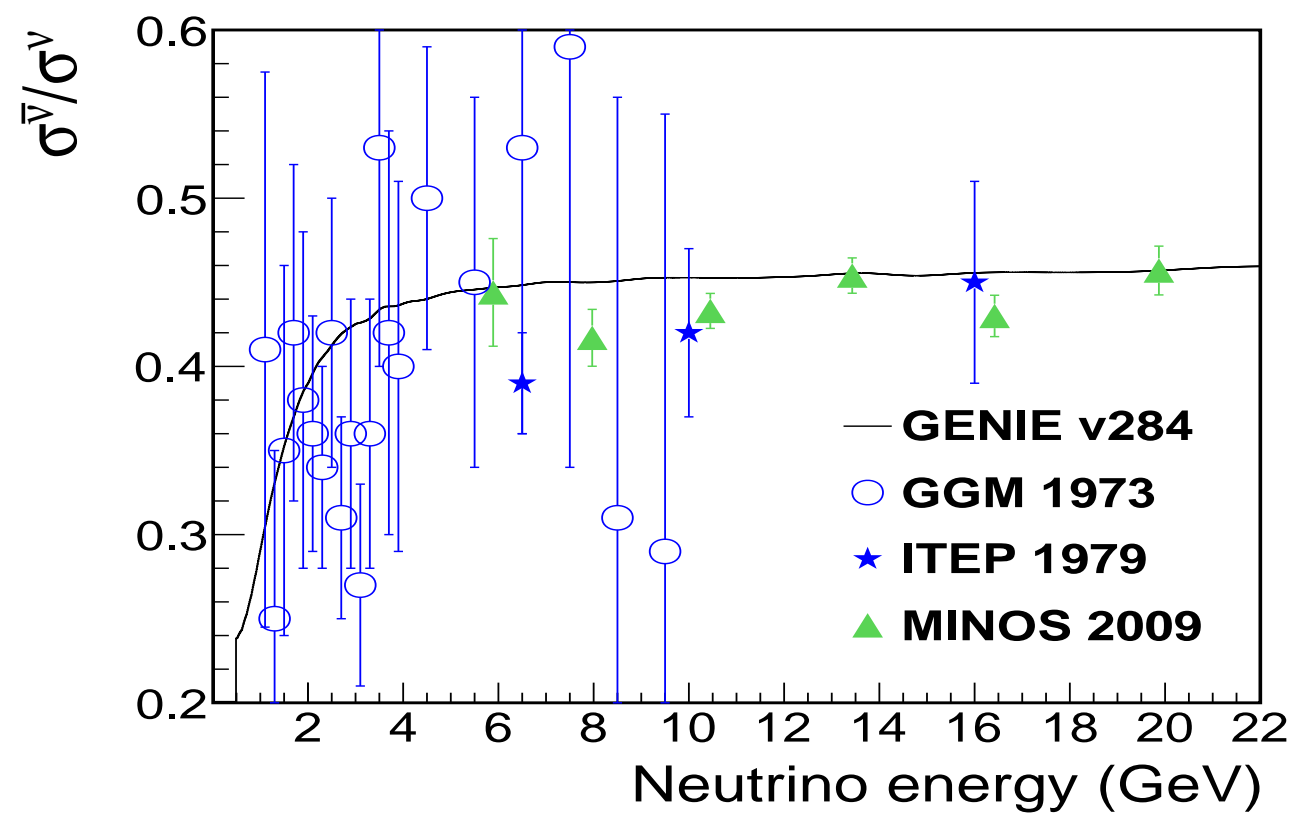

Figure 8: Measurements of antineutrino to neutrino charged-current cross section ratio. 


\subsection{THE NUMI BEAM, MINER $\nu$ A DETECTOR AND MINOS NEAR DETECTOR}

The MINER $\nu \mathrm{A}$ detector [8] is designed to measure cross sections for neutrino interactions on nuclei in the energy region of $<20 \mathrm{GeV}$. The Neutrinos at the Main Injector (NuMI) [5] neutrino beam at Fermilab provides an intense source of neutrinos and antineutrinos for MINOS, MINER $\nu \mathrm{A}$, and NOvA experiments [47]. The MINER $\nu \mathrm{A}$ and MINOS Near Detector (ND) are $1 \mathrm{~km}$ downstream of the target in NuMI Hall $100 \mathrm{~m}$ underground. In this chapter, we describe the NuMI beam, MINER $\nu \mathrm{A}$ detector and MINOS ND and their calibrations.

\subsection{THE NUMI BEAM}

Neutrinos are produced by directing a $120 \mathrm{GeV}$ proton beam onto a graphite target. Mesons are produced and then focused by two magnetic horns and enter a helium filled "decay pipe" where a fraction of them decay into neutrinos. At the end of decay volume follows a hadron absorber, after which only muons and neutrinos are left in the beam. The muon and neutrino beam then passes throughout $240 \mathrm{~m}$ thick rocks, where most of the muons are absorbed. Fig. 9 shows a schematic of the NuMI beamline.

The proton beam originates from an $\mathrm{H}^{-}$ions source accelerated in the Linac to $400 \mathrm{MeV}$. The Booster synchrotron [5] then converts them to protons and accelerates them to $8 \mathrm{GeV}$ in about 67 $\mathrm{ms}$, the protons are then directed into the Main Injector ring, which accelerates the protons to 120 $\mathrm{GeV}$. Protons are delivered to NuMI target every $2.2 \mathrm{~s}$ in a single turn extraction, which lasts about $10 \mu s$ and store about $4.2 \times 10^{13}$ protons. The protons are separated into six time batches as shown in Fig. 13.

The mesons produced from the graphite target are focused by two magnetic horns downstream 


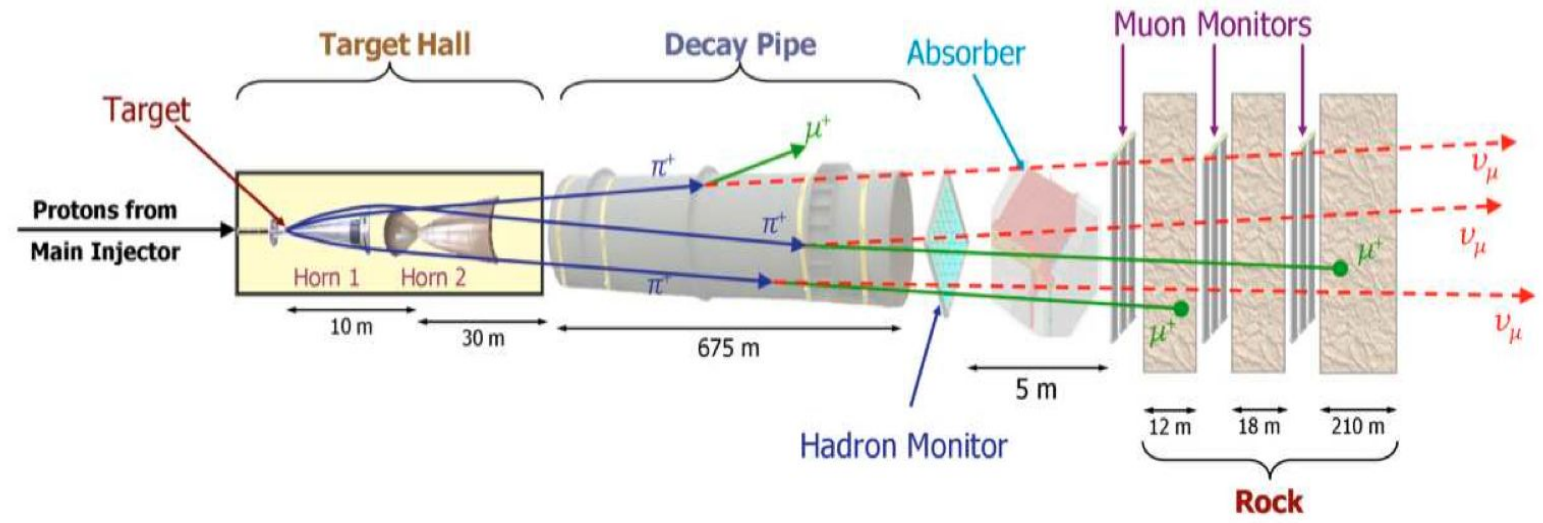

Figure 9: Schematic of the NuMI Beam(taken from [5] ).

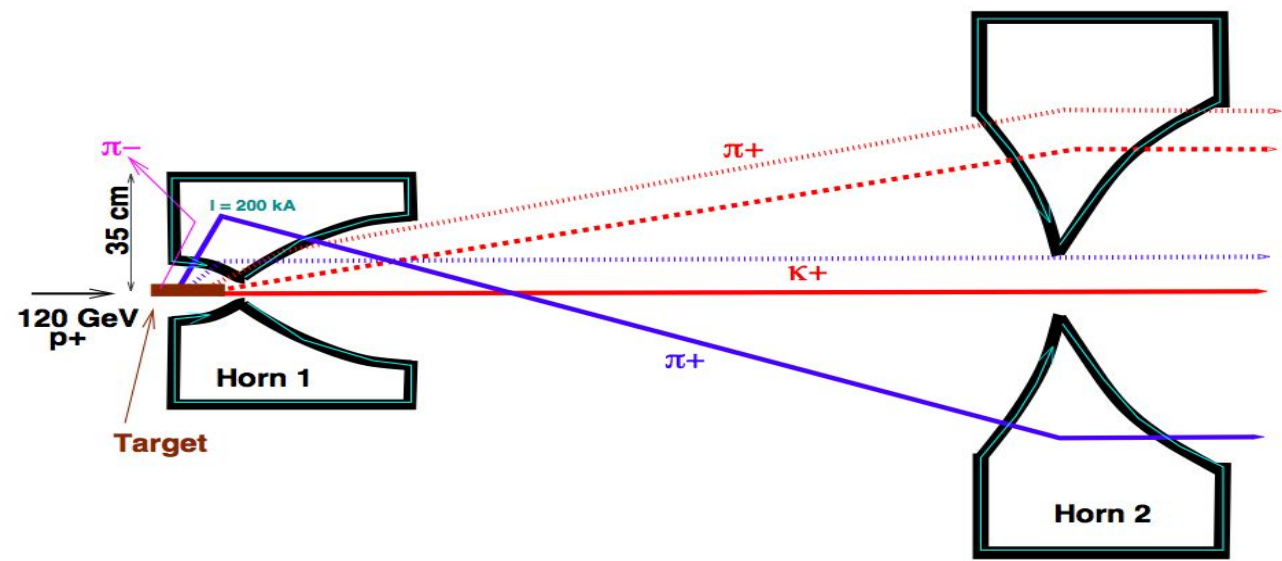

10 meters

Figure 10: A illustration of two possible trajectories inside two magnetic horns. The first horn focus either positive or negative hadrons, and the second horn further focus them (taken from [5]).

of the target. The horns are pulsed with a current to bend charged mesons to the proton beams path.The target to horn distance and separation between two horns are flexible, which can change the spectrum of focused particles, thereby change the energy distribution of neutrinos. The polarity 


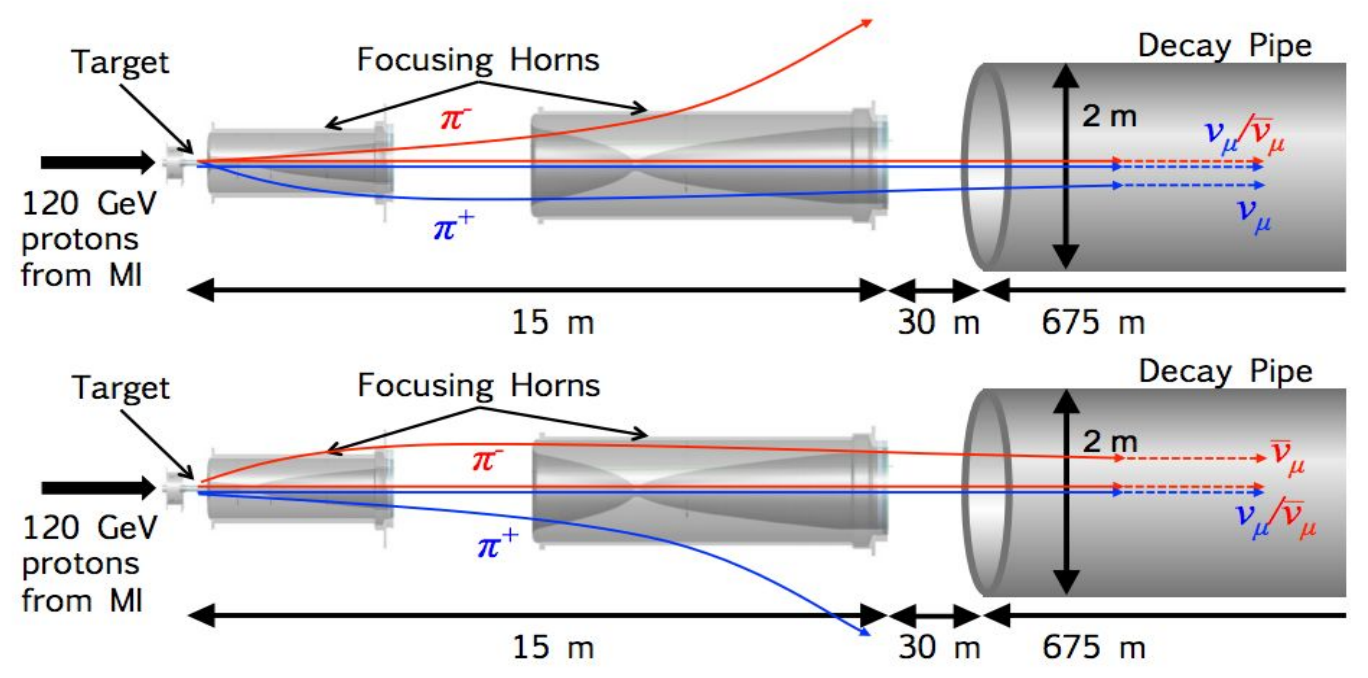

Figure 11: A illustration of forward horn current (top) and reverse horn current (bottom) modes (taken from [6]).

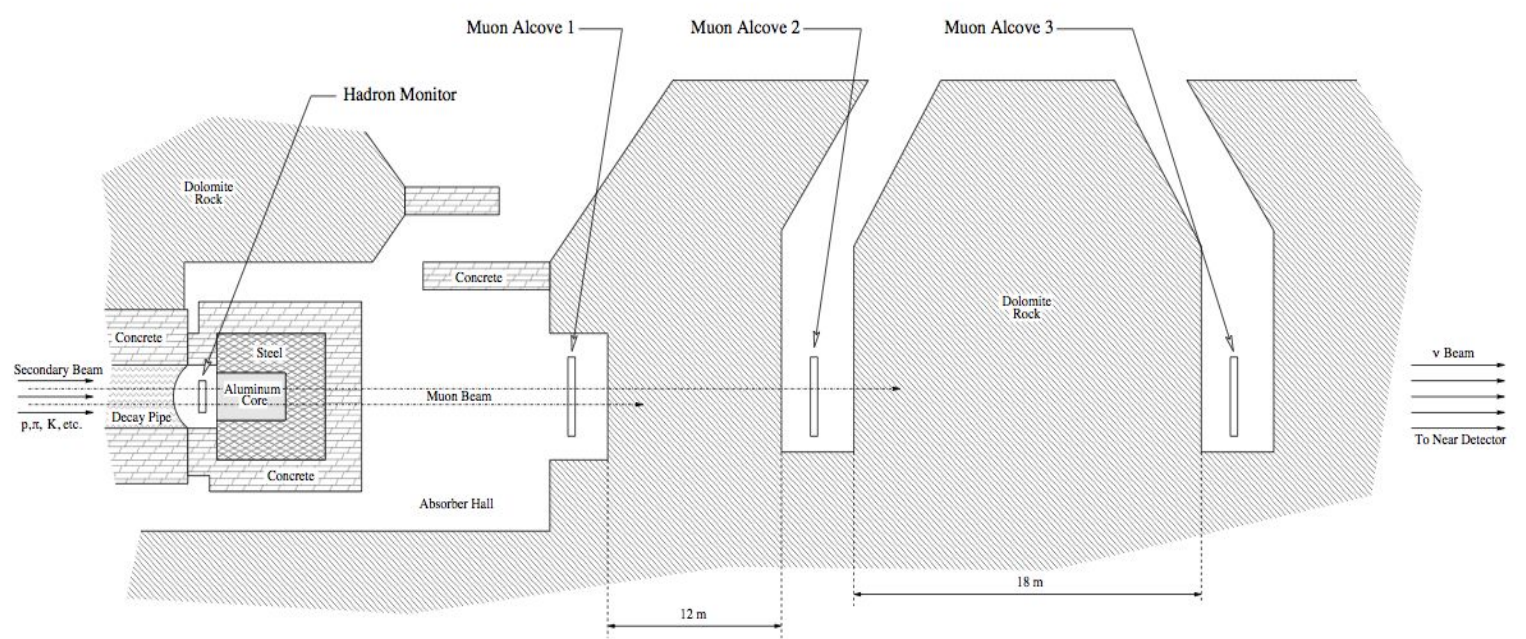

Figure 12: A sketch of the area downstream of the decay pipe, which includes the hadron absorber, the hadron monitor, and the three muon monitors (taken from [5]). 


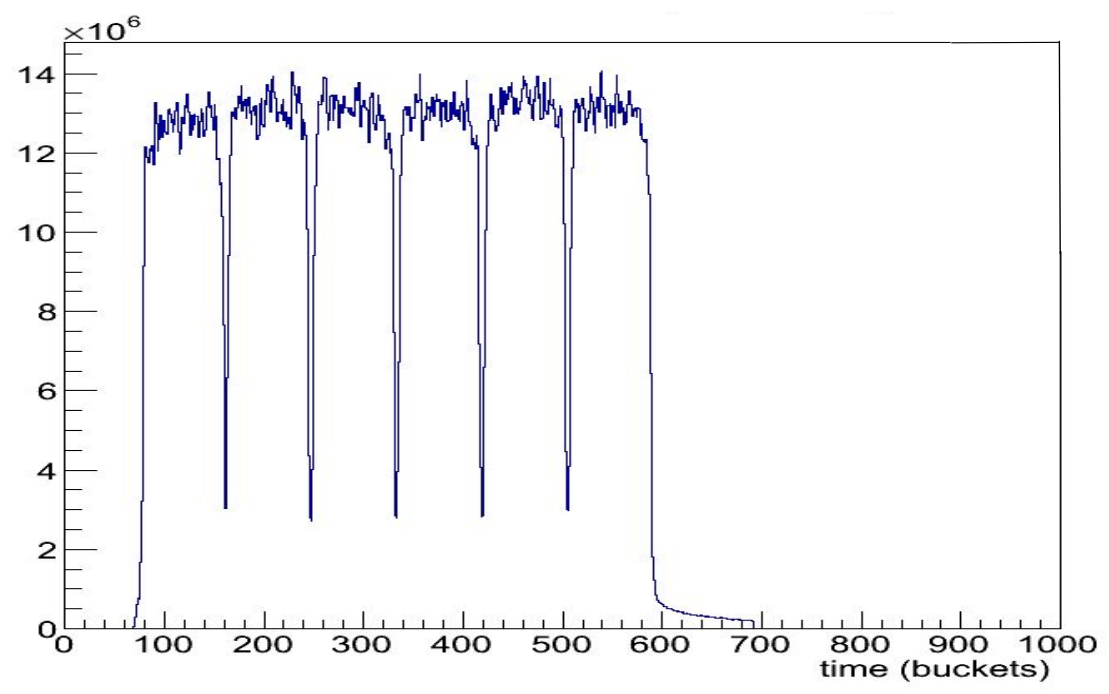

Figure 13: A illustration of six time batches structure from MINOS online monitoring page.

of the horn current can be reversed, thus allow the hadron focusing to produce neutrino-enhanced or antineutrino-enhanced beam as shown in Fig. 11.

After being focused by the magnetic horns, mesons enter the decay pipe, where they decay into charged leptons, neutrinos and other mesons. The decay pipe is downstream of the target, it is $675 \mathrm{~m}$ long and filled with helium.

Just downstream of the decay pipe is an absorber made of aluminum, steel and concrete structure. Undecayed mesons and uninteracted protons are stopped in the absorber, which also serves to protect ground water from irradiation. The $240 \mathrm{~m}$ of rock and dirt between the absorber and the MINOS ND hall, will range out the remaining muons in the NuMI beam. Muons created by neutrino interactions in the rock region upstream of $\operatorname{MINER} \nu \mathrm{A}$ are still present, these are referred as "rock muon" later in this thesis.

To make a neutrino-enhanced beam, the polarity of the horn current is set to focus positive mesons, which is called "forward horn current" (FHC). In order to make an antineutrino-enhanced beam, the polarity of the horn current is reversed to "reversed horn current" (RHC) mode, which focuses negative charges. Fig. 14 shows the energy spectrum of neutrino and antineutrino beams and their backgrounds used in the simulated Monte Carlo samples. RHC antineutrino-enhanced 
mode contain larger wrong-sign background comparing with FHC neutrino-enhanced mode.

The fluxes shown were produced with the FTFP BERT model of GEANT4 9_2_p03 [48] and significantly constrained with hadron production data (NA49 [49]). Uncertainties on these data are propagated into flux [7].
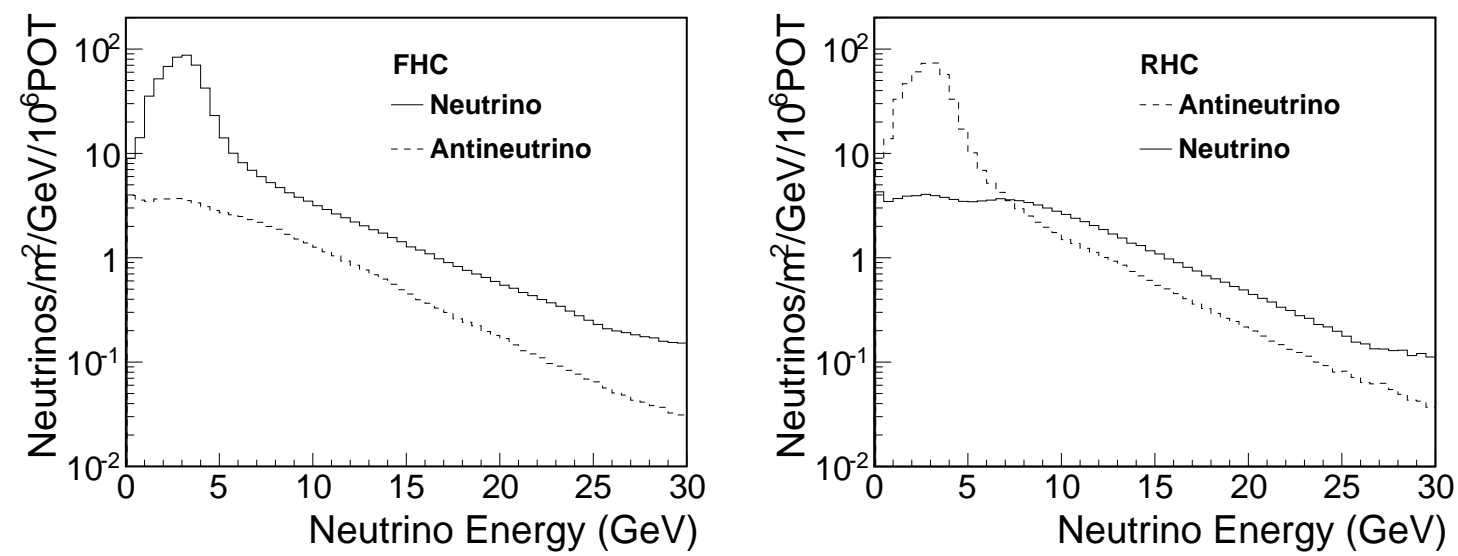

Figure 14: Predicted fluxes in FHC and RHC beam modes obtained using the "PPFX gen-2 thin" flux described in [7].

\subsection{MINER $\nu$ A DETECTOR}

Fig 15 shows the front view of MINER $\nu$ A detector. It is a hexagonal cylinder with an apothem of $1.7 \mathrm{~m}$ and $5 \mathrm{~m}$ in length. Fig. 16 shows the side view of the detector. The downstream-most plane of MINER $\nu \mathrm{A}$ is $2 \mathrm{~m}$ upstream of the front face of the MINOS ND. There is a steel shield and veto wall upstream of the main detector, which shields the low energy particles, photons and also tags the rock muons. A liquid helium target (not used in this analysis) is placed between the veto wall and the main detector. There are two major subsections called the inner detector (ID) and outer detector (OD). 


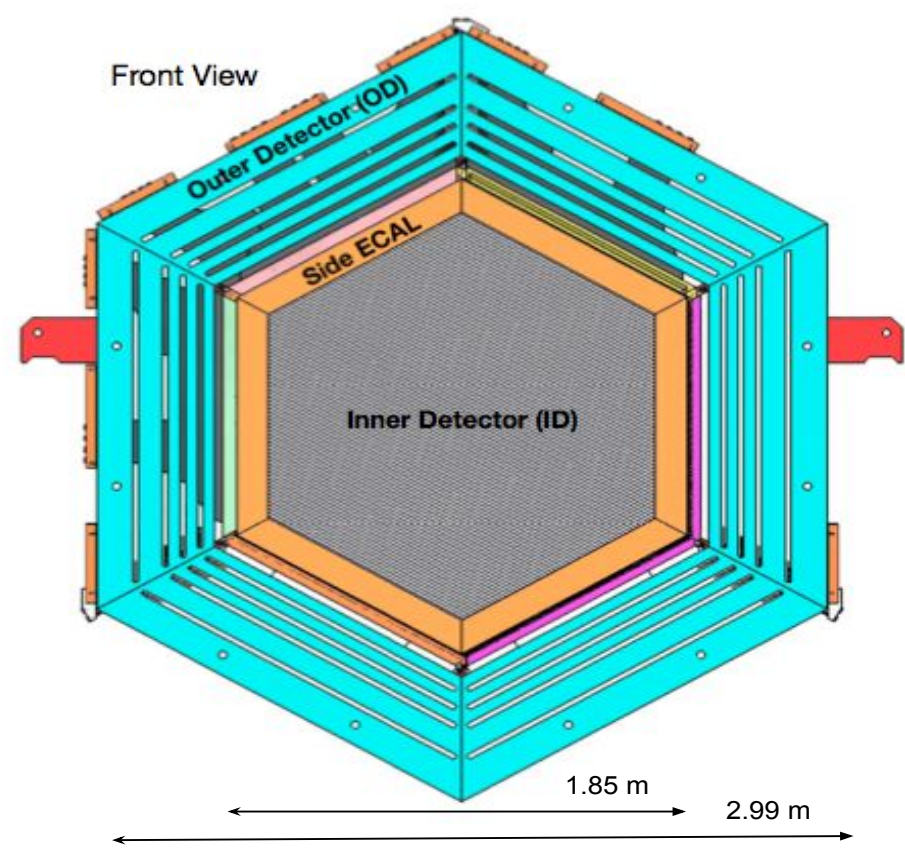

Figure 15: Front view of MINER $\nu \mathrm{A}$ detector, taken from [8].

\subsubsection{Inner Detector}

The inner detector is divided into four regions, which are the nuclear target region, active tracking region (tracker), downstream electromagnetic calorimeter (ECAL) and downstream hadronic calorimeter (HCAL) region.

2.2.1.1 Scintillator Planes $M I N E R \nu A$ uses scintillator planes which are $1.7 \mathrm{~cm}$ thick and 3.3 $\mathrm{cm}$ wide and have 127 triangular strips, each scintillator has a hole at the triangle center, $0.85 \mathrm{~cm}$ above the widest edge [8]. Two planes of scintillator are mounted in one frame, which is called a module. The scintillator planes are installed in three different directions, as shown in Fig. 17, which allows precise tracking reconstruction by three dimension tracking.

MINER $\nu \mathrm{A}$ uses extruded plastic scintillator in the tracker region of the inner detector. The scintillator is made from Dow Styron $663 \mathrm{~W}$ polystyrene $\left((\mathrm{CH})_{8 n}\right)$ and doped with 1\% 2,5diphenyloxazole (PPO) and 0.03\% 1,4-bis(5-phenyloxazol-2-yl) benzene (POPOP) by weight. The 


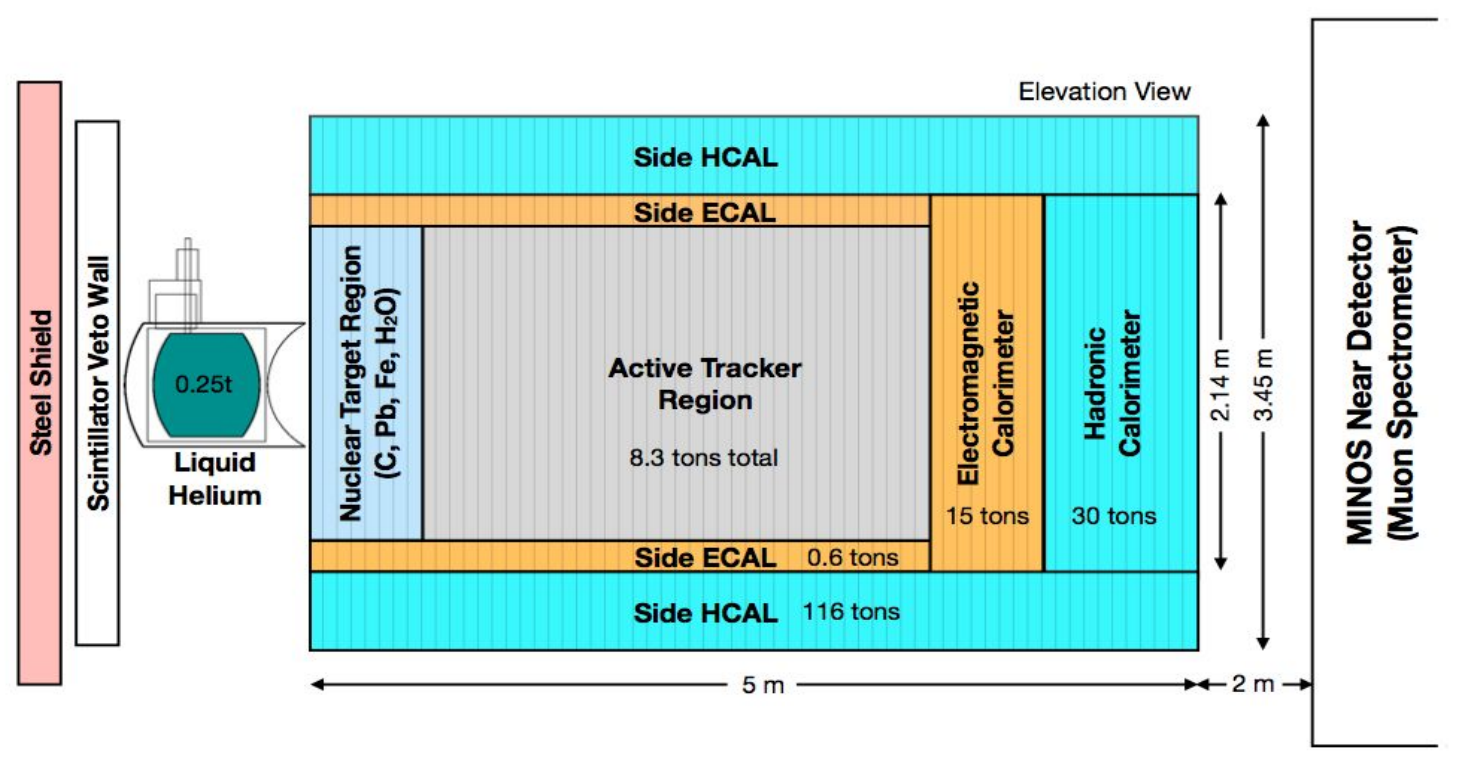

Figure 16: Side view of MINER $\nu \mathrm{A}$ detector, showing the nuclear target region, tracker region, surrounding calorimeters and MINOS ND, taken from [8].

strips are co-extruded with a $0.25 \mathrm{~mm}$ white coating based on $15 \% \mathrm{TiO} 2$ (by weight) in polystyrene to improve internal reflection [8].

2.2.1.2 Nuclear Targets The nuclear target region is located at the upstream of the detector. It consists of 22 tracking modules and 5 passive targets. Passive targets are numbered from 1 to 5 from upstream to downstream, and are built with four types of nuclear targets: $\mathrm{Fe}, \mathrm{C}, \mathrm{Pb}$ and $\mathrm{He}$. Target 1 and 2 consists of iron and lead, with direction of iron and lead flipped. Target 3 consists of iron, lead and carbon, Target 4 consist of lead only and is thinner than other targets. Target 5 consists of iron and lead and has half the thickness of target 1. Nuclear targets are not used in this measurement.

2.2.1.3 Active Tracker region This measurement uses neutrino and antineutrino interactions in this active tracker region. This region consists of 62 modules, each of which includes two scintillator planes. It is the main region to reconstruct tracks of charged particles as they go through 
the detector.
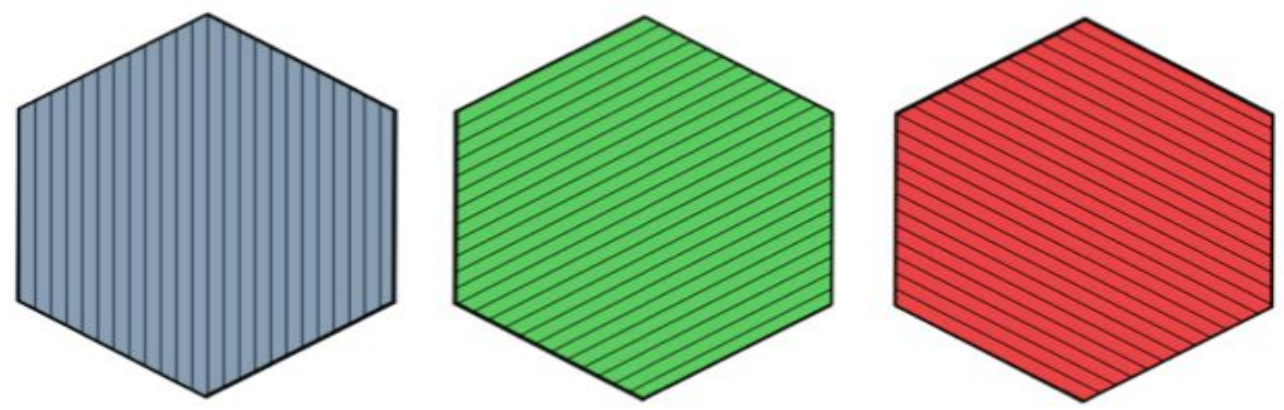

Figure 17: From left to right are $\mathrm{X}, \mathrm{U}$ and $\mathrm{V}$ scintillator plane orientations. The lines show the direction of scintillator strips, taken from [8].

2.2.1.4 Electromagnetic Calorimeter(ECAL) The ECAL follows immediately downstream of the tracker region as shown in Fig. 16. It consists of 10 modules, each of which includes two scintillator planes. The surface of each plane is covered with $0.2 \mathrm{~cm}$ thick lead sheet which act as an absorber. The sides of the nuclear target and tracker regions are also surrounded by ECAL modules (side ECAL).

2.2.1.5 Hadronic Calorimeter(HCAL) The HCAL begins at the downstream of the ECAL (and also surrounds the side ECAL). It consists of 20 modules, each of which includes a 1 inch thick steel absorber and one scintillator plane.

\subsubsection{Outer Detector}

The outer detector (OD) is the blue region shown in Fig. 15. It surrounds the inner detector and has a width of $56 \mathrm{~cm}$. It serves to tag and possibly contain particles exiting from the side of detector. It is composed of a steel frame with scintillator strips instrumented. It also serves as the supporting structure. The OD is not used in energy reconstruction but it is used to veto muons with wide angles in this analysis. 


\subsubsection{Light Collection}

A wavelength shifting (WLS) fiber is embedded in the hole of each strip, which is at the triangle center, $0.85 \mathrm{~cm}$ above the widest edge. The WLS fiber is $1.2 \mathrm{~mm}$ in diameter, $175 \mathrm{ppm} \mathrm{Y-11}$ doped, S-35, multiclad fiber made by the Kuraray corporation. One end of the WLS fiber is mirrored in order to minimize the light loss. WLS fiber collects the blue light, shifts it to green. The shifted green light is reflected internally in the fiber and passed along to the end of the strip and directed out of the scintillator by a Fujikura-DDK connector, which connects to cables containing eight clear optical fibers transmitting light from the WLS fibers to the PMT boxes above the detector. Each connector groups eight fibers. Eight connectors are plugged into each Photomultiplier tubes(PMTs) box for a total of 64 fibers per box. Light collected from scintillators are amplified into a measureable signal by 507 Hamamatsu Photonics H8804MOD-2 multi-anode PMTs (which are the same PMTs used by MINOS) [8]. Each PMT has 64 pixels distributed in $8 \times 8$ array on a 2 $\mathrm{cm} \times 2 \mathrm{~cm}$ grid, called "cookie". The fibers from adjacent scintillator strips are arranged to be not from adjacent pixels in the PMT, which minimize the effect of cross talk. Each PMT is mounted onto a base circuit board that contains the Cockroft-Walton high voltage generator. The operating high voltage is generally around $820 \mathrm{~V}$. The PMT and base circuit board are installed inside a 2.36 mm thick steel cylindrical box which provides shieldings from MINOS ND's residual magnetic fields. The PMT boxes are mounted onto racks directly above the detector.

\subsubsection{Data Acquisition}

PMT signals are read by front end boards (FEBs), which are attached to the PMT boxes. FEBs store timing and charge for all 64 channels in six TriP-t chips, which are controlled by a FieldProgrammable Gate Array (FPGA). Up to ten FEBs are daisy-chained to form a readout chain. The readout chain is connected at both ends to a custom VME module called the Chain Read Out Controller-Ethernet (CROC-E), which serves up to 4 FEB chains. The CROC-Es receive timing and trigger commands from a custom module, the CROC-E Interface Module (CRIM), which services four CROC-Es. The CROC-Es and CRIMs are divided into two VME crates along with a CAEN V2718 crate controller. Timing information, like the NuMI and MINOS triggers, is communicated among VME modules by CRIM. 
MINER $\nu \mathrm{A}$ reads out the entire detector for $16 \mu$ s for a trigger signal from the end of each 10 $\mu s$ spill of the NuMI beam. The additional $6 \mu s$ ensure that any delayed detector activity, such as from Michel electrons from muon decays, are recorded.

\subsubsection{Calibration}

Calibration of MINER $\nu$ A data is described in detail in [8]. Fig. 18 shows the schematic of the components in each readout channel which requires calibration. Four effects must be included to convert ADC to energy deposited:

- The scintillation light is attenuated as it travels long the WLS.

- The digit is also attenuated in the clear optical fiber.

- Photons reaching the PMT are converted into photoelectrons, which are amplified by the dynode chain.

- The readout charge is digitized on the FEB to an ADC.

The full calibration chain is described as

$$
E_{i}=\left[C(t) \cdot S_{i}(t) \cdot \eta_{i}^{a t t} \cdot e^{l_{i} / \lambda_{\text {clear }}} \cdot G_{i}(t) \cdot Q_{i}(A D C)\right] \times A D C_{i},
$$

where $E_{i}$ is the estimated energy deposited in channel $i, C(t)$ is overall energy scale for the entire

detector and $S_{i}(t)$ is the relative correction factor for channel $i, \eta_{i}^{a t t}$ is the correction factor for attenuation within the scintillator strip, $e^{l_{i} / \lambda_{\text {clear }}}$ is the correction factor for attenuation within the clear optical fiber of length $l_{i}, G_{i}(t)$ is the gain of PMT dynode chain, and $Q_{i}(A D C)$ is the ADCto-charge conversion factor for the FEB channel used to read out strip $i$.

2.2.5.1 Ex situ calibrations Some calibrations are measured before the final assembly of the detector.

- Module mapper. The goal is to determine the optical attenuation a function of position along the scintillator strip. This test uses two Cs-137 radioactive sources, the sources are moved in a pre-defined scan pattern over each detector module. $\eta_{i}^{a t t}$ in Eq. 2.1 is determined for each strip. 


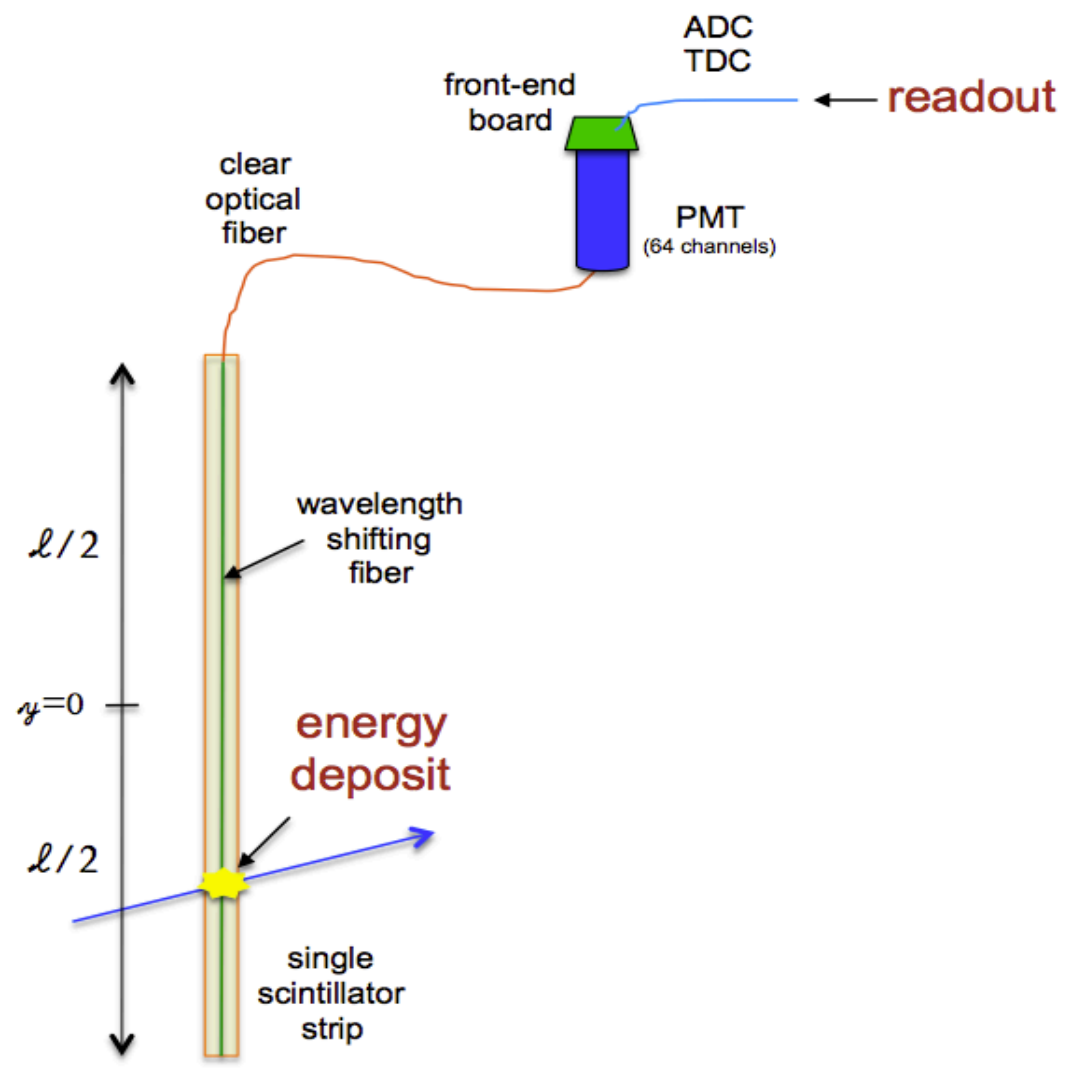

Figure 18: Schematic of a single optical channel in MINER $\nu$ A, taken from [8].

- FEB response. FEBs were tested before the installation in the detector, which includes the measuring its response to charge in low, medium and high gain channels. The conversion of charge to ADC count was measured and $Q_{i}$ in Eq. 2.1 is determined from this calibration.

- PMT testing. All PMTs were tested before installed in the optical boxes for efficiency, dark noise and cross-talks. Six PMTs are mounted onto cookies on the light-tight test stand and tested at once. 
2.2.5.2 In situ calibrations In situ calibration constants are measured during the run with the operating detector using rock muons that occur during the beam spill, or special calibration triggers (pedestal or light injection) taken between beam spills.

- Pedestal monitoring. The pedestal is measured by reading out the detector when there is no light. Pedestal runs are taken between NuMI triggers. The mean of the ADC distribution, which coming from the noise of cosmic rays and PMT dark current, is subtracted during calibration.

- PMT gain monitoring. To monitor the fluctuations in PMT gain for each channel in the detector, data with light injection triggers is taken between NuMI spills. Two LED light injection boxes are used, they are tuned so that one photoelectron is generated in each channel. The gain is calculated from the difference of RMS between the pedestal ADC and light injection ADC.

- Scintillator plane alignment. The scintillator planes can be misaligned either by shifting by the same distance to one side, or rotating about the $z$ axis. Rock muons are used in this calibration since they travel along the beam direction. The overall shift is measured by comparing the peak of energy depositions. The rotation parameter is determined from the overall shift of planes.

- Relative strip-to-strip response variations. A strip-to-strip calibration is performed to account for the variations in light level between different strips. The resulting constant for each strip is obtained by measuring the energy per length of rock muons which exit from the back face of the detector, the variation in the peak of energy per length distribution gives the stripto-strip constant.

- Timing calibration. This calibration corrects for time offset due to the transport in optical fiber, time slewing and position of FEB in a chain. It uses rock muons as well.

- Cross-talk. It is identified as the signal measured in channels that were produced by rock muons in another channel in same PMT. The energy distribution of cross-talk is compared to the simulation to obtain a correction.

2.2.5.3 Absolute energy scale The absolute energy scale is described in terms of a muon equivalent unit (MEU) of energy deposited, which is well understood in the scintillator planes. Rock muons are used for this calibration. The calibration is the ratio of the MEU extracted from the fit 
of energy deposition distribution of data to simulation. Fig. 19 shows a comparison of data and simulated MEU used to do the calibration and the fit of energy distribution, the peak of which is set as the MEU value.
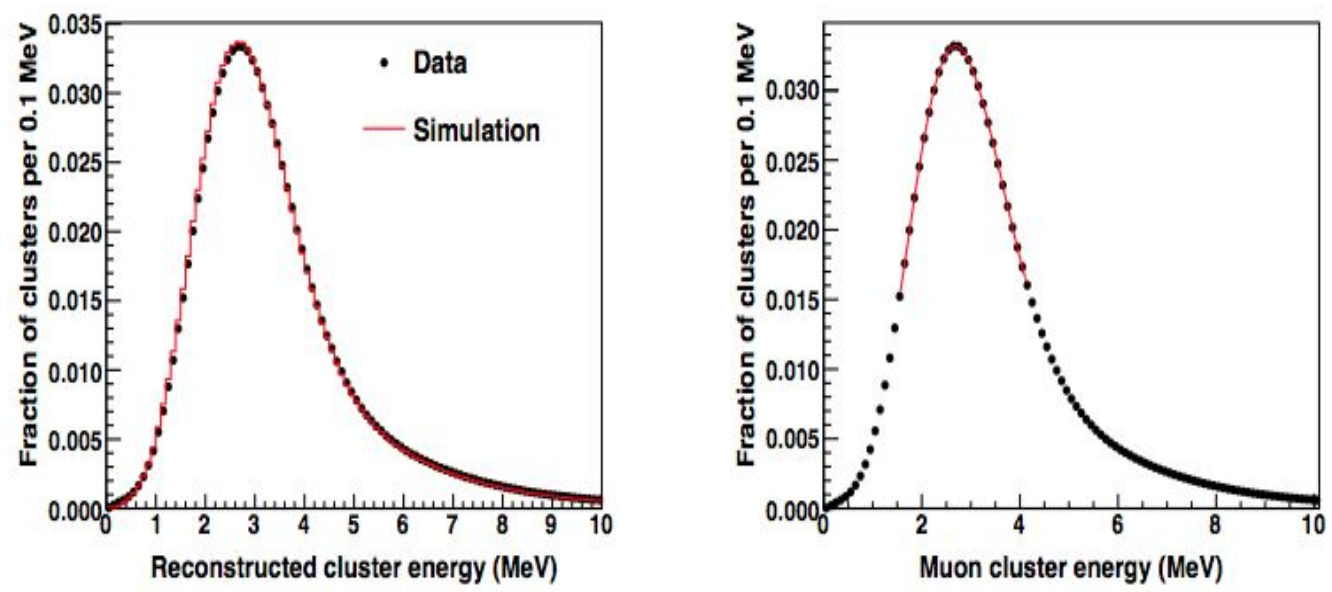

Figure 19: Absolute energy scale calibration using MEU. Left one shows the comparison of data to MC MEU used to do the calibration, right one shows the fit of energy distribution, from which the peak is set as the MEU value. (taken from [8]).

2.2.5.4 Hadron energy scale Low energy hadrons and electrons are calibrated using a mockup of the MINER $\nu \mathrm{A}$ detector (with 40 planes) placed in a test beam at Fermilab. Incoming beam of protons, pions and electron are used to measure detector response of each particle. The reconstructed shower energy (discussed in Chapter 4) uses measured single particle response as systematic uncertainty on each type of particle in this analysis as described in Chapter 7. Details of MINER $\nu \mathrm{A}$ test beam detector can be found in [50]. 


\subsection{MINOS NEAR DETECTOR}

The Main Injector Neutrino Oscillation Search (MINOS) [51] is a two detector neutrino oscillation experiment. MINOS Near Detector (ND) sits $2 \mathrm{~m}$ downstream of MINER $\nu$ A along the NuMI beam. It is magnetized and serves as a muon spectrometer to measure the momentum of muons exiting from the downstream end of MINER $\nu$ A detector.

Fig. 20 shows the schematic of the MINOS ND. It is has a squashed octagonal shape, which is $4.8 \mathrm{~m}$ in width, $3.8 \mathrm{~m}$ in height and $16.6 \mathrm{~m}$ in length. It consists of 282 steel planes, each of which is $2.54 \mathrm{~cm}$ thick, of which 152 are instrumented with $4.1 \mathrm{~cm}$ wide and $1 \mathrm{~cm}$ thick scintillator planes.
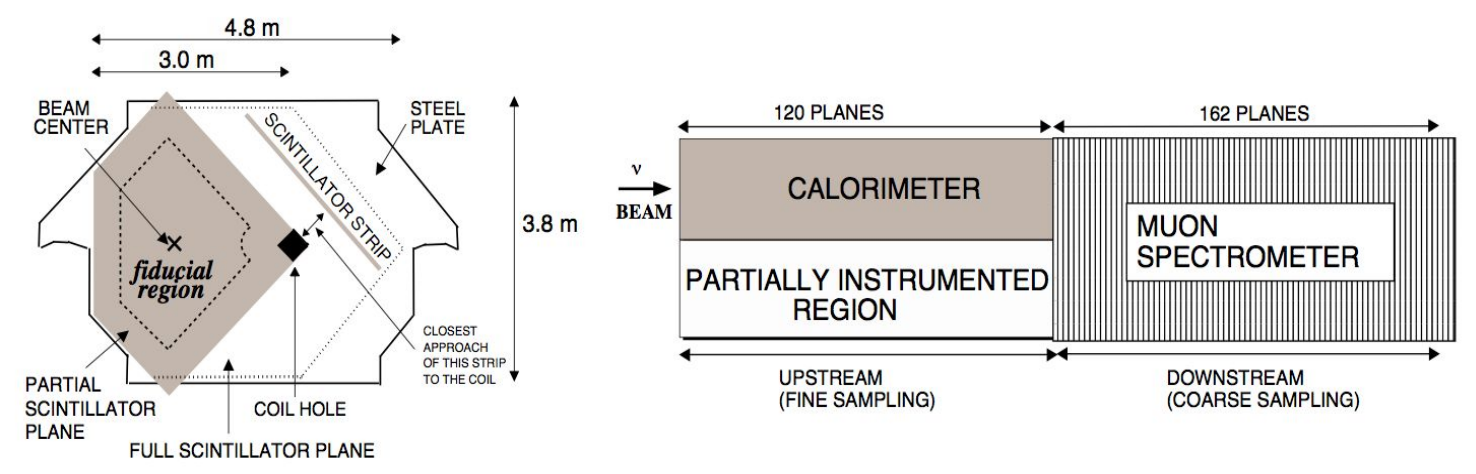

Figure 20: Schematic of the MINOS ND. Left shows the front view of MINOS ND, the shaded area is partially instrumented active scintillator plane and the dashed line within shows the fiducial region. Right shows the longitudinal view of the calorimeter and muon spectrometer, taken from [8].

The upstream 120 planes of MINOS ND, which are partially-instrumented scintillator planes (with 64 scintillator strips), is the "calorimeter region". The downstream 162 planes, which have no partially instrumented planes and every fifth plane is fully-instrumented (with 96 scintillator strips), is the "muon spectrometer". Naming of two regions is for the purpose of MINOS experiment itself.

The magnetic field of MINOS ND is generated by an aluminum coil through the whole detector along the beam direction. The coil is located $1.5 \mathrm{~m}$ from the beam center, it carries a current of 40000 A-turns with each 48 turns carrying $833 \mathrm{~A}$. A toroidal field with $1.3 \mathrm{~T}$ is generated, which 
causes tracks produced by charged particles to curve. The direction of curvature measures the particle's charge sign, and the radius of curvature is used to determine the particle momentum.

MINOS ND measures muon momentum by range $\left(P_{\text {range }}\right)$ or curvature $\left(P_{\text {curv }}\right)$ based on whether it stops inside or exits the detector. For muon tracks which exit MINER $\nu$ A and stop inside MINOS ND, the muon momentum, $P_{\text {range }}$, is determined by the length of track segment in MINOS ND. The uncertainty of momentum reconstructed by range is about $2 \%$, which includes the $1 \%$ disagreement of reconstructed and true muon energy above $1 \mathrm{GeV}$ obtained from the MINOS Monte Carlo event sample, the $1 \%$ uncertainty of energy loss in the simulation and the less than $1 \%$ uncertainty due to detector mass model which comes from the thickness and position of planes. (The coil hole geometry is also simulated in the model [18]).

For muon tracks which exit MINOS ND, curvature is used to measured the momentum. The curvature of a muon track is defined as [8].

$$
\frac{1}{R}=\frac{0.3 B}{P}
$$

in which, $B$ is the magnetic field in $\mathrm{kG}, P$ is the momentum component perpendicular to the field, and $R$ is the radius of curvature in $\mathrm{cm}$. The uncertainty of muon momentum reconstructed by curvature is determined by the term $1 / P_{\text {curv }}-1 / P_{\text {range }}$ as shown in Fig. 21 . The uncertainty obtained is $0.6 \%$ for muons with $P_{\text {curv }}>1 \mathrm{GeV}$ and $2.5 \%$ for muons with $P_{\text {curv }}<1 \mathrm{GeV}$. A summary of muon energy scale can be found in Chapter 6, where we discuss the systematic uncertainty of this measurement. 

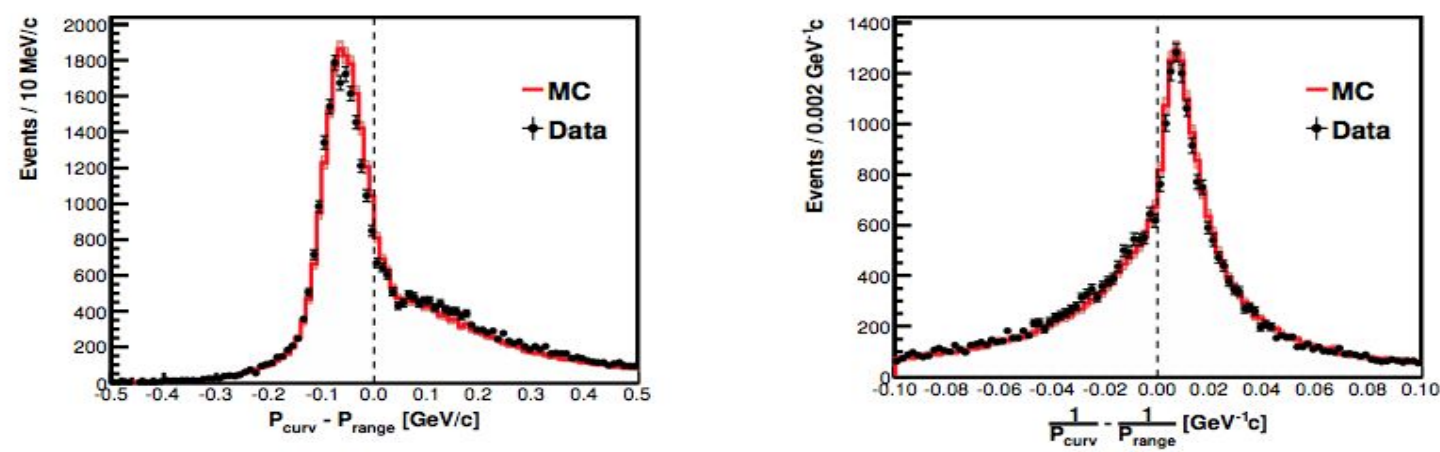

Figure 21: Left shows $P_{\text {curv }}-P_{\text {range }}$ for inclusive events with muon stops in the calorimeter of MINOS, right shows $1 / P_{\text {curv }}-1 / P_{\text {range }}$ for the same events (taken from [8]). 


\subsection{GENERATOR MODELS}

In this chapter, we describe the cross section models of two generators, GENIE [1] and NuWro [52], which are both used to calculate the model-dependent corrections in this analysis. GENIE 2.8.4 is the default model used to generate Monte Carlo (MC) samples of neutrino interactions. It uses relativistic Fermi gas (RFG) to model the nuclei and it includes interaction components of quasielastic scattering, resonance production, deep inelastic scattering, coherent production, etc. However, GENIE 2.8.4 can not well describe the data with low momentum transfer processes, such as quasi-elastic and $\Delta(1232)$ resonance production region. Recent study [13] has suggested that we include a "Hybrid piece" into GENIE 2.8.4 to better describe data at low momentum transfer region. Therefore, we introduce a modified GENIE model called "GENIE Hybrid", and use this model to obtain the model-dependent corrections for our primary results. In order to study the cross section model dependence of this measurement, we use NuWro, as an alternative event generator, to re-calculate model-dependent corrections and obtain alternative results. Sec. 3.1 presents the GENIE Hybrid model, including GENIE 2.8.4 and the Hybrid piece. Sec. 3.2 describes the NuWro model.

\subsection{GENIE HYBRID}

In this section, we first describe the model components in GENIE 2.8.4, including QEL, RES, DIS, $\mathrm{COH}$ and tuning of the overlapping region. Then, we introduce the Hybrid piece, which includes Random Phase Approximation(RPA) [9], Meson Exchange Current(MEC or “2p2h") [10] and pion reweighting[53]. Finally, we discuss the final state interaction (FSI) model in GENIE 2.8.4. 


\subsubsection{GENIE 2.8.4}

3.1.1.1 Quasi-Elastic Scattering (QEL) Quasi-elastic scattering is modeled using LlewellynSmith model as described in Sec. 1.3.4. BBBA2005 [54] parametrization of electromagnetic form factors is used, of which there are two remaining form factors. The pseudo-scalar form factor has the form as suggested by partially conserved axial current hypothesis, which is written as

$$
F_{p}\left(q^{2}\right)=\frac{2 M^{2} F_{A}\left(q^{2}\right)}{M_{\pi}^{2}-q^{2}},
$$

and the axial form factor $F_{A}\left(q^{2}\right)$ is given by a dipole form

$$
F_{A}\left(q^{2}\right)=\frac{F_{A}(0)}{\left(1-\frac{q^{2}}{M_{A}^{2}}\right)^{2}}
$$

The value of $F_{A}(0)$ is determined from neutron beta decay measurements to be -1.267 . The $M_{A}$ parameter is set to0.99 $\mathrm{GeV} / \mathrm{c}^{2}$ [1]. For nuclear targets, a suppression factor is applied in the RFG model, which requires that the momentum of the outgoing nucleon is larger than the Fermi momentum $k_{F}$, which is $0.221 \mathrm{GeV} / \mathrm{c}^{2}$ for nucleons in carbon 12. A summary of related parameters are given in Tab. 5 .

\begin{tabular}{cc}
\hline parameter & value \\
\hline axial mass $M_{A}$ & $0.99 \mathrm{GeV}$ \\
vector mass $M_{V}$ & $0.84 \mathrm{GeV}$ \\
proton anomalous magnetic moment & 2.7930 \\
neutron anomalous magnetic moment & -1.913 \\
axial form factor at $Q^{2}=0$ & $-1.267 \mathrm{GeV}^{2}$ \\
\hline
\end{tabular}

Table 5: Parameters for QEL model in GENIE 2.8.4. 
3.1.1 Baryon Resonance Production (RES) Baryon resonance production is included using Rein-Sehgal model [54]. There are 16 resonances considered, which are $P_{22}(1234), P_{11}(1450)$, $D_{13}(1525), S_{11}(1540), S_{31}(1620), S_{11}(1640), P_{33}(1640), D_{13}(1670), D_{15}(1680), F_{15}(1680), P_{11}(1710)$, $D_{33}(1730), P_{13}(1740), P_{31}(1920), F_{35}(1920), F_{37}(1950), P_{33}(1960)$ and $F_{17}(1970)$. Two resonances in the original paper are not included, since they are listed as ambiguous in the PDG tables. The interference between neighboring resonances is not included. In the calculation of differential cross section, lepton mass terms are not included, but the effect of the lepton mass on the phase space boundaries is considered. Resonance axial vector mass $M_{A}$, defined in Eq. 1.53, is set to 1.12 $\mathrm{GeV} / c^{2}$ by default according to the global fit in Ref. [55]. While the vector mass $M_{V}$ in Eq. 1.53 is set to $0.84 \mathrm{GeV} / c^{2}$. Constant $\Omega$ in FKR model, as defined in Eq. 1.51, for baryon excitation is set to 1.05 .

\subsubsection{Deep Inelastic Scattering (DIS) Deep inelastic scattering is defined as non-resonance} inelastic scattering in GENIE. It is calculated in an effective leading order model, and modifications suggested by Bodek and Yang are used [21]. In this model, a new scaling variable and modifications to the low $Q^{2}$ parton distributions are used to account for the higher twist and target mass corrections. This scaling variable is defined as

$$
\xi=\frac{2 x\left(Q^{2}+M_{f}^{2}+B\right)}{Q^{2}\left[1+\sqrt{1+4 M^{2} x^{2} / Q^{2}}\right]+2 A x},
$$

where $M_{f}$ is the mass of quark in the final state. The parameter $A$ accounts for the higher order QCD terms in the form of an enhanced target mass term, while $B$ accounts for the initial state quark transverse momentum and final state quark effective $\Delta M_{f}$. Cross section is modeled using quark parton model. The default parameters are determined based on the GRV98 LO parton distributions [56]. $K$ factors are defined to describe the low $Q^{2}$ data,

$$
K\left(Q^{2}\right)=\left[1-G_{D}^{2}\left(Q^{2}\right)\right] \times \frac{Q^{2}+C_{v 2}}{Q^{2}+C_{v 1}},
$$

where $G_{D}$ is the proton elastic form factor. An overall scale factor 1.032 is applied in order to agree with the measured value $\left(0.675 \mathrm{~cm}^{2} / \mathrm{GeV}\right)$ at $100 \mathrm{GeV}$. This scale factor needs to be recalculated if cross section models in GENIE are changed. Parameters used in DIS structure function model are listed in Tab. 6. 


\begin{tabular}{ccc}
\hline BY model parameter & Name in GENIE & Value \\
\hline$A_{H T}$ & BY-A & 0.538 \\
$B_{H T}$ & BY-B & 0.305 \\
$C_{s u}$ & BY-CsU & 0.363 \\
$C_{s d}$ & BY-CsD & 0.621 \\
$C_{V 1 u}$ & BY-Cv1U & 0.291 \\
$C_{V 2 u}$ & BY-Cv2U & 0.189 \\
$C_{V 1 d}$ & BY-Cv1D & 0.202 \\
$C_{V 2 d}$ & BY-Cv2D & 0.255 \\
$X_{0}$ & BY-X0 & -0.00817 \\
$X_{1}$ & BY-X1 & 0.0506 \\
$X_{2}$ & BY-X2 & 0.0798 \\
\hline
\end{tabular}

Table 6: Constants in the Bodek-Yang (BY) DIS structure function model and in the Bodek-Yang GRV-LO-98 PDF used in GENIE v2.8.4.

3.1.1.4 Other Contributions Other than the components we just talked about, the largest contribution considered is coherent pion production $(\mathrm{COH})$, which is modeled using the Rein-Sehgal model [57]. A modified PCAC formula which includes lepton mass terms used in the implementation, since it requires a small momentum transfer. In Rein-Seghal model, it begins with PCAC form at $Q^{2}=0$, assuming a dipole dependence for non-zero $Q^{2}$, and then calculates the cross section from measured data on total and pion scattering.

There are a few other channels contributing to the inclusive cross section in GENIE 2.8.4, but are much smaller. These includes quasi-elastic charm production which is modeled using Kovalenko local duality inspired model [58] and tuned to NOMAD data; deep-inelastic charm production which is modeled using [59] and inclusive inverse muon decay which is modeled using [60] which takes into account of all one-loop radiactive corrections. 


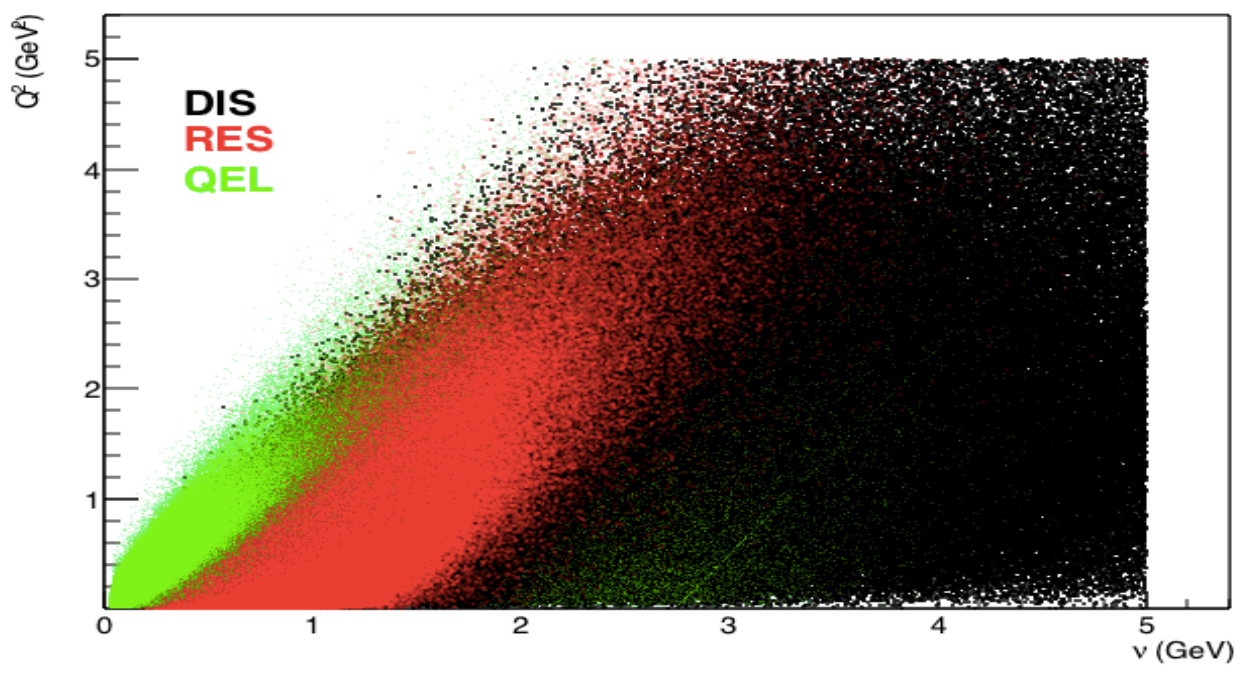

Figure 22: $Q^{2}$ vs hadron energy distributions for DIS, RES and QEL, obtained from MC sample generated with GENIE 2.8.4. QEL dominates at low $Q^{2}$ and low hadronic energy, RES is at the transition region between QEL and DIS, and DIS dominates at high hadronic energy region. 
3.1.1.5 Cross section model tuning The parameters in QEL, RES and DIS models are determined by the global fits [54] [55]. In order to model the transition region in GENIE [1], the total cross section can be written as

$$
\sigma^{t o t}=\sigma^{Q E L} \oplus \sigma^{R E S} \oplus \sigma^{D I S}
$$

The total inelastic differential cross section is computed as

$$
\frac{d^{2} \sigma^{i n e l}}{d Q^{2} d W}=\frac{d^{2} \sigma^{R E S}}{d Q^{2} d W}+\frac{d^{2} \sigma^{D I S}}{d Q^{2} d W}
$$

in which

$$
\frac{d^{2} \sigma^{R E S}}{d Q^{2} d W}=\Sigma_{k}\left(\frac{d^{2} \sigma^{R S}}{d Q^{2} d W}\right)_{k} \cdot \Theta\left(W_{c u t}-W\right)
$$

where $k$ runs over all the available baryon resonances in GENIE, $\left(\frac{d^{2} \sigma^{R S}}{d Q^{2} d W}\right)_{k}$ is the differential cross section of $k$ th resonance as predicted by Rein-Seghal model, $W_{c u t}$ is a configurable parameter. The DIS term is defined so that in the region $W<W_{c u t}$, the RES/DIS mixture agrees with inclusive cross section, exclusive 1-pion and 2-pion data,

$$
\frac{d^{2} \sigma^{D I S}}{d Q^{2} d W}=\frac{d^{2} \sigma^{D I S, B Y}}{d Q^{2} d W} \cdot \Theta\left(W-W_{c u t}\right)+\frac{d^{2} \sigma^{D I S, B Y}}{d Q^{2} d W} \cdot \Theta\left(W_{c u t}-W\right) \cdot \Sigma_{m} f_{m}
$$

where $m$ refers to the multiplicity of hadronic system. The factor $f_{m}$ is defined as $f_{m}=R_{m} P_{m}^{\text {had }}$, where $R_{m}$ is a tunable parameter and $P_{m}^{h a d}$ is the probability the DIS final state hadronic system multiplicity equals to $m$. The parameters are tuned to be $W_{c u t}=1.7 \mathrm{GeV}, R_{2}(\nu p)=R_{2}(\bar{\nu} n)=$ $0.1, R_{2}(\nu n)=R_{2}(\bar{\nu} p)=0.13$, and $R_{m}=1.0$ for all $m>2$ interactions, based on electron scattering data [61] and neutrino structure function data ${ }^{1}$ [18]. Fig. 22 shows the $Q^{2}$ vs $\nu$ distribution for generated charged-current neutrino events. QEL dominates where $\nu<1 \mathrm{GeV}$, QEL and RES overlap, as $\nu$ increases, DIS becomes the only contributor.

\footnotetext{
${ }^{1}$ This parameter is set to 0.3 in GENIE v2.8.4, it is scale to $43 \%$ based on the study in [53]
} 


\subsubsection{Hybrid Only Model Components}

3.1.2.1 Random Phase Approximation (RPA) RPA effect describes the suppression of cross section due to long-range nucleon-nucleon correlations. The strong interaction between nucleons makes the electroweak couplings change from their free nucleon values. Fig. 23 shows the contribution of adding RPA on the W-self energy. In one particle one hole (1p1h) interactions, the RPA corrections do not only depend on the different terms of the nucleon currents, but also on the particular component of the hadronic tensor which is being renormalized [9]. In order to implement the RPA effect, a two-dimensional suppression factor in (q0, q3) shown in Fig. 24 is applied to the QE events generated by GENIE 2.8.4. The suppression factor is calculated in Ref. [62], it is defined as the ratio of cross sections bewteen the model with RPA effect and the model without RPA effect for neutrino (antineutrino) carbon interaction at $3 \mathrm{GeV}$.

3.1.2.2 MEC 2 particles 2 holes (" $2 \mathrm{p} 2 \mathrm{~h}$ ") interaction refers to the process that excites two particles out of the nucleus leaving two holes, it contributes to the cross section with two nucleons in the final state. The Feynman diagrams in Fig. 25 shows an example of 2p2h event that have an an internal pion exchange. Since these processes produce two particle final states at low momentum, it enhances the predicted hadron energy. As shown in Fig. 26, 2p2h has large contribution between QEL and $\Delta$ overlapping region, as well as the peak region of both QEL and $\Delta$.

We take the $E_{\nu}=2.5 \mathrm{GeV}$ neutrino and antineutrino samples as an example to further explain the kinematic distributions of $2 \mathrm{p} 2 \mathrm{~h}$ events. Tab. 7 shows the number of non- $2 \mathrm{p} 2 \mathrm{~h}$ events, $2 \mathrm{p} 2 \mathrm{~h}$ events in generated samples. For 100,000 generated neutrino (antineutrino) events, about 6\% ( $9 \%$ ) are predicted to be 2 p2h events. Fig. 27 shows hadron energy and muon energy distributions, for generated neutrino and antineutrinos samples. For both neutrino and antineutrino, $2 \mathrm{p} 2 \mathrm{~h}$ events populate the $E_{\text {had }}<1.2 \mathrm{GeV}\left(E_{\mu}>1.3 \mathrm{GeV}\right)$ region, which enhance the peak of hadron energy distribution at $0.5 \mathrm{GeV}$, where $\mathrm{QE}$ and $\Delta$ overlaps. 


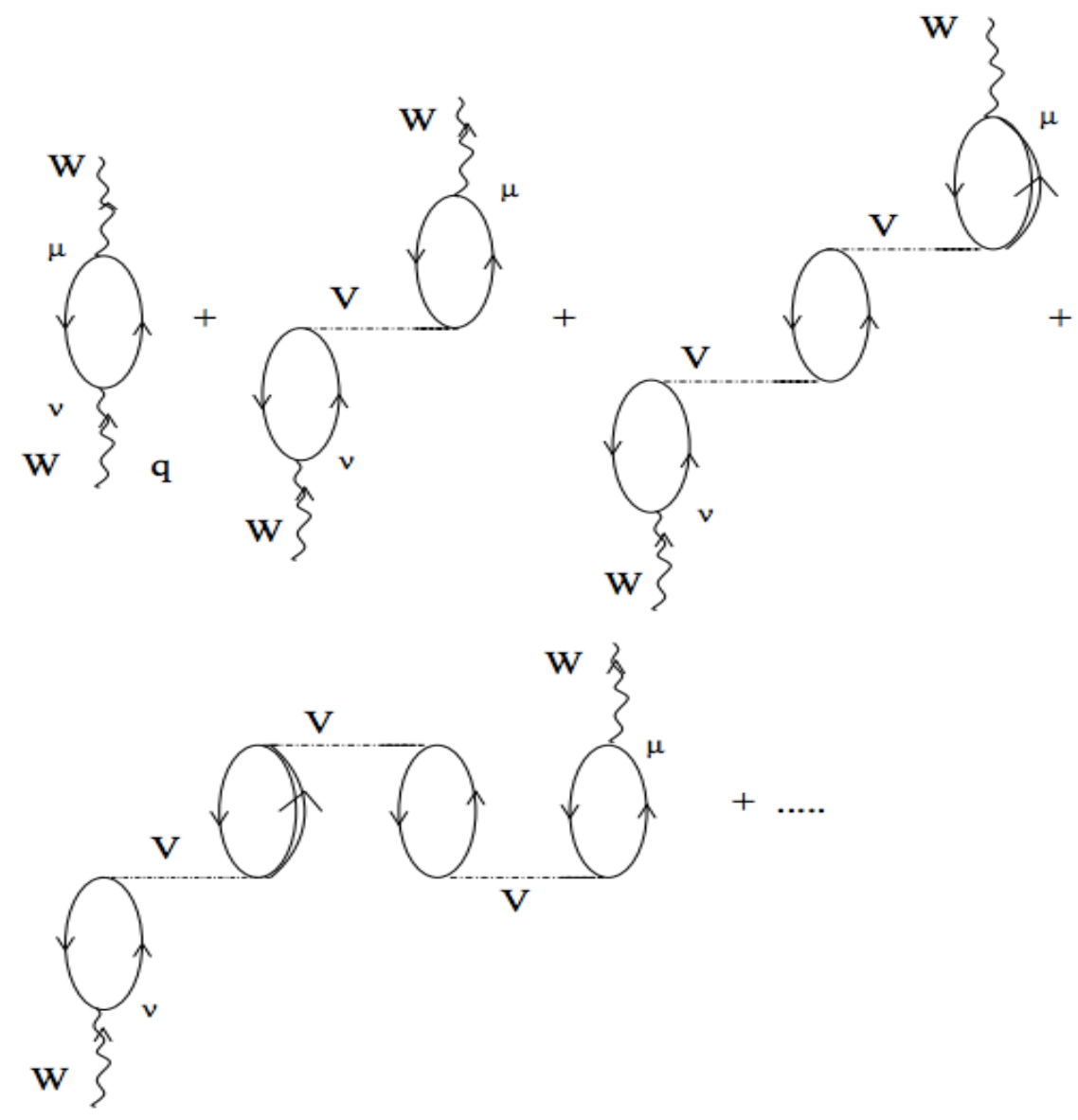

Figure 23: Set of irreducible diagrams responsible fore RPA effect in $1 \mathrm{p} 1 \mathrm{~h}$ contribution to W-self energy, taken from [9]. 

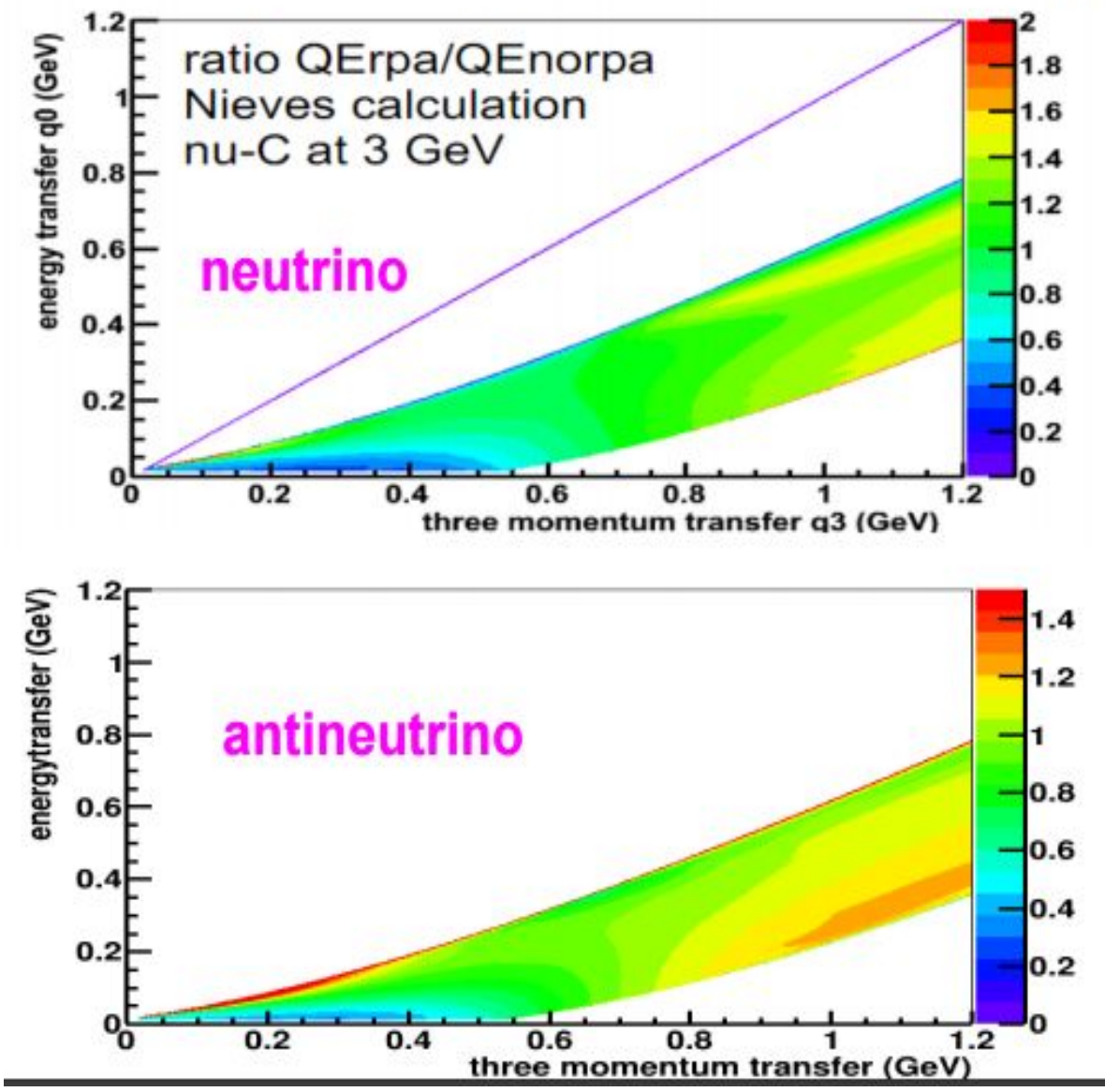

Figure 24: Two-dimensional correction in $(\mathrm{q} 0, \mathrm{q} 3)$ which is formed from the ratio of cross sections between the model with and without RPA effect. 


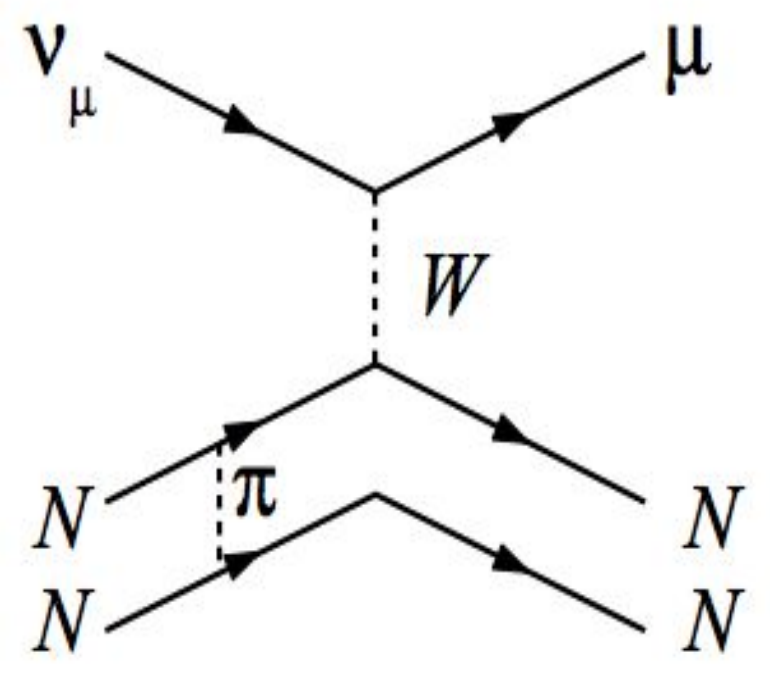

Figure 25: Feynman diagram for 2p2h (MEC) interaction.
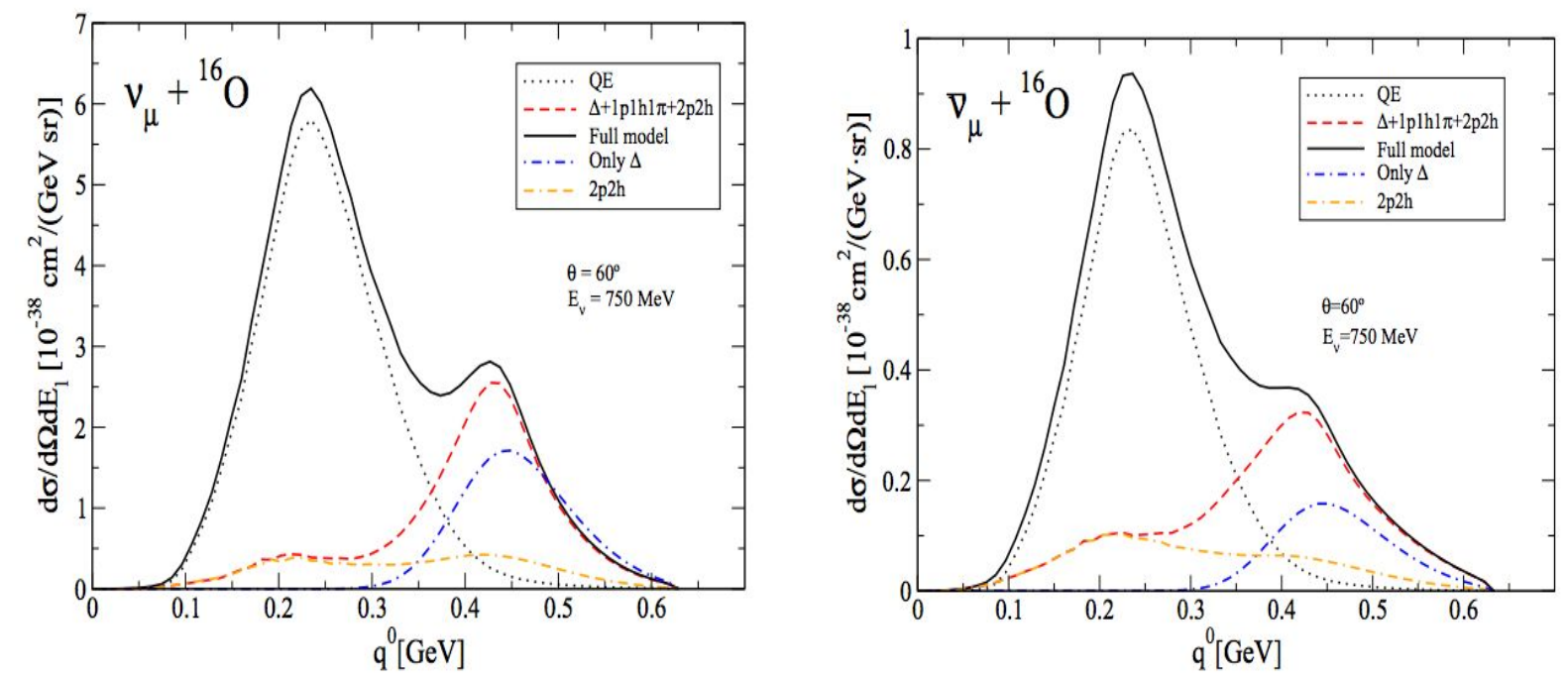

Figure 26: Neutrino and antineutrino CC differential cross section in oxygen, at 60 degrees of scattering angle and neutrino energy at $0.75 \mathrm{GeV}$, taken from [10]. 


\begin{tabular}{|c|c|c|}
\hline flag & neutrino & antineutrino \\
\hline non-2p2h & 94286 & 90797 \\
2p2h & 5714 & 9203 \\
total & 100000 & 100000 \\
\hline
\end{tabular}

Table 7: $2 \mathrm{p} 2 \mathrm{~h}$ contributions in generated sample at $E_{\nu}=2.5 \mathrm{GeV}$.

3.1.2.3 Pion Reweighting We reduce the GENIE single pion non-resonant component with initial state $\nu+n$ for neutrino(or $\bar{\nu}+p$ for antineutrino) by $57 \%$. Recent study has shown that it improves agreement with observed deuterium data [53].

\subsubsection{Final State Interaction (FSI) Model}

FSI changes the spectrum of reconstructed neutrino energy, which is essential for this measurement. Tab. 32 and Tab. 36 show the parameters in the FSI model.

Hadrons produced in the nuclear region (within $4 R$, where $R$ is the nucleus radius) may reinteract on their way out of the nucleus, which changes the kinematic observables. The GENIE model for FSI simulates the re-scattering of pions in the nucleus. It steps through the nucleus in steps of $0.1 \mathrm{fm}$, where for each step, it calculates the probability of the particles' interactions, which is related to the density of nucleus and the cross section of intranuclear reactions.

Final state interactions change the number and type of final state hadrons. There are four types of interactions simulated, including elastic scattering, inelastic scattering, absorption and charge exchange. For elastic scattering, pion remains the same charge after scattering and the final state nucleus is in its ground state, while for inelastic scattering, pion scatters and the nucleus is left in an excited state. The pion can also be absorbed in the nucleus with no pions in the final state. In addition, there might be a hadron of the same type but different charge in the final state, which is called charge exchange. 

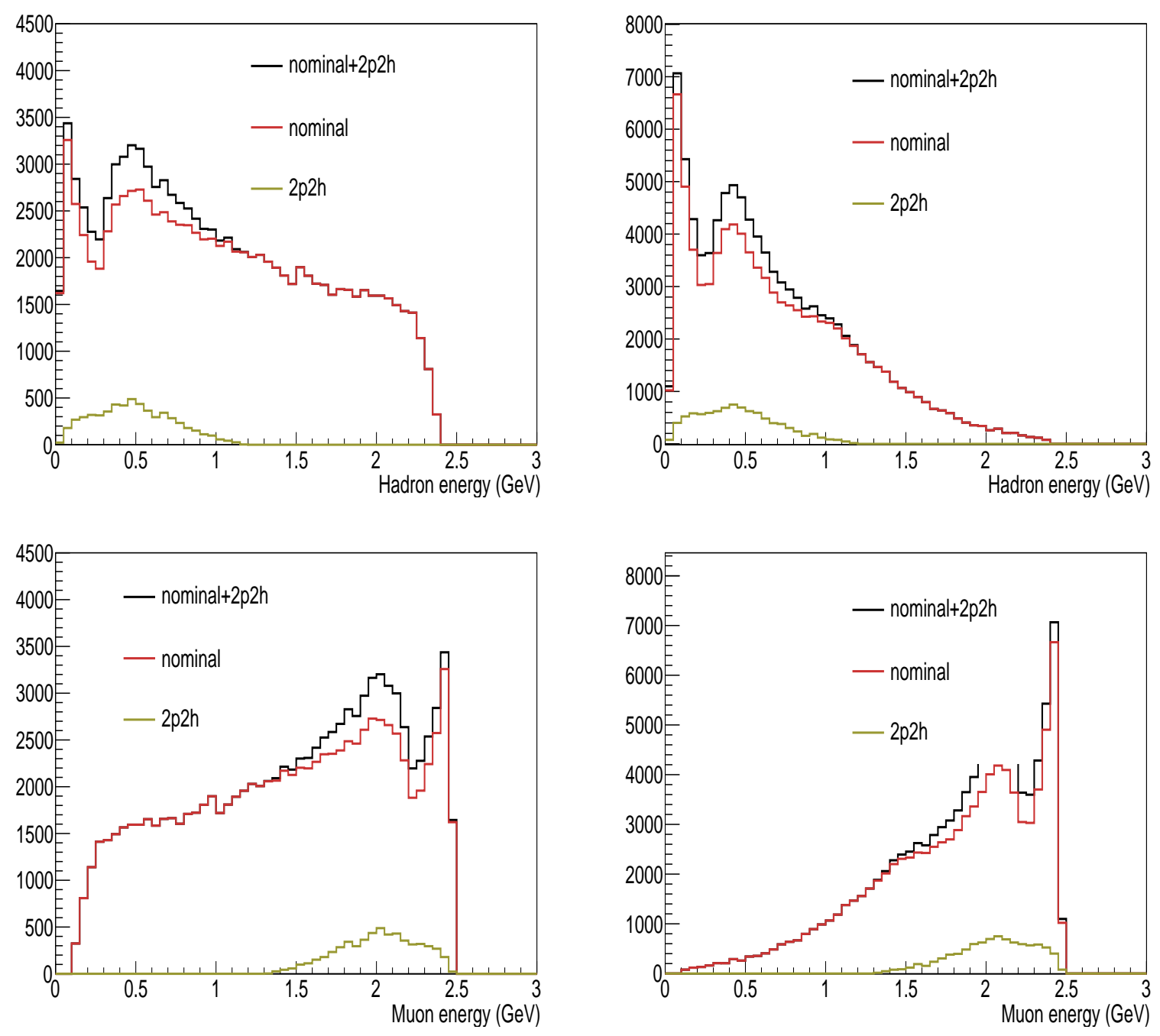

Figure 27: Hadron energy distributions of generated MC neutrino events with $E_{\nu}=2.5 \mathrm{GeV}$ for neutrino (top left) and antineutrino (top right) and muon energy distributions of those events for neutrino (bottom left) and antineutrino (bottom right). Nominal is from GENIE v2.8.4 model, $2 \mathrm{p} 2 \mathrm{~h}$ is from Nieves model.

\subsection{NUWRO}

Here we discuss the model differences between NuWro [52] and GENIE. Default parameters of NuWro model are shown in Tab. 8 ( with RFG nuclear model). The four basic charged-current 
interaction channels, QEL, RES, DIS and COH [11], which are in common with GENIE. However, they are implemented differently.

\begin{tabular}{|c|c|c|}
\hline Parameter & Value & Description \\
\hline qel_vector_ff_set & 2 & Electromagnetic form factors parametrization: BBBA05 \\
\hline qel_axial_ff_set & 1 & Axial form factors parametrization: dipole form \\
\hline qel_strange & 0 & Turn on strange quark contribution to the NC axial form factors \\
\hline qel_strangeEM & 0 & Turn on strange quark contribution to the NC vector form factors \\
\hline delta_s & -0.15 & $g_{A}^{s}$ \\
\hline qel_cc_axial_mass & $1200 \mathrm{MeV}$ & The axial mass value for charged current form factors \\
\hline qel_nc_axial_mass & $1350 \mathrm{MeV}$ & The axial mass value for neutral current form factors \\
\hline qel_s_axial_mass & $1200 \mathrm{MeV}$ & The axial mass value used in the dipole strange form factor \\
\hline qel_rpa & 0 & Do not use RPA \\
\hline delta_FF_set & 1 & $\Delta$ production form factor: dipole form \\
\hline pion_axial_mass & $0.94 \mathrm{GeV}$ & The axial mass value used in pion production form factor. \\
\hline pion_C5A & $1.19 \mathrm{GeV}$ & The $C_{5}^{A}$ value used in pion production form factor. \\
\hline spp_precision & $500 \mathrm{MeV}$ & Controls the precision in RES-DIS boundary region \\
\hline res_dis_cut & $1600 \mathrm{MeV}$ & Boundary of RES-DIS transition \\
\hline coh_mass_correction & 1 & Turn off Rein-Seghal correction to $\mathrm{CC}$ coherent pion production \\
\hline coh_new & 1 & improved implementation of coherent pion production \\
\hline mec_kind & 1 & Transverse Enhancement model \\
\hline mec_ratio_pp & 0.6 & The fraction of mixed initial nucleon pairs for $\mathrm{CC}$ interaction \\
\hline
\end{tabular}

Table 8: Parameters used in NuWro generator.

- QEL: NuWro uses the same form factor parametrization for QEL as GENIE (described in Sec. 3.1.1.1), but with different axial mass values, which causes large difference in the kinematic corrections, as described later in Chapter 7.

- RES: In NuWro, RES is defined as the region with $\mathrm{W}<1.6 \mathrm{GeV}$, where $\mathrm{W}$ is the invariant hadronic mass. The dominant contribution comes from the single pion production mediated by 
the $\Delta(1232)$ resonance. Different from GENIE's model described in Sec. 3.1.1.2, $\Delta$ resonance in NuWro is described by the form factors as in [63]. The axial mass value and axial vector transition form factor $C_{5}^{A}$ value are shown in Table. 8.

- DIS: DIS channel is defined as the region with $\mathrm{W}>1.6 \mathrm{GeV}$ in NuWro. The total cross sections are also evaluated using the Bodek-Yang model described in Sec 3.1.1.3. However, NuWro uses modified parton distribution functions GRV94 for DIS interaction, while GENIE uses GRV98LO.

- Modeling of Overlap Region: Unlike the model tunning of GENIE (described in Sec. 3.1.1.5), in NuWro, exclusive cross sections for single pion production (SPP) channels are given by

$$
\frac{d \sigma^{S P P}}{d W}=\frac{d \sigma^{\Delta}}{d W}(1-\alpha(W))+\frac{d \sigma^{D I S}}{d W} F^{S P P}(W) \alpha(W)
$$

in which $F^{s p p}$ is the percentage of the given single pion production channel within the overall DIS cross section. $\alpha(W)$ defines the transition between two single pion production models, which accounts for the non-resonance background.

- MEC: In NuWro, MEC is modeled using transverse enhancement model [12], which is also different from GENIE (described in Sec. 3.1.2.2). Inclusive cross section data is fitted as a sum of four components: the longitudinal QE contribution, the transverse QE contribution, a transverse excess (TE), and the contribution of inelastic pion production. The transverse enhancement ratio is defined as

$$
R_{T}=\frac{Q E_{\text {tran }}+T E}{Q E_{\text {tran }}} .
$$

Fig. 30 shows the values of $R_{T}$ as a function of $Q^{2}$. The data are parametrized as

$$
R_{T}=1+A Q^{2} e^{-Q^{2} / B}
$$

with $A=6.0$ and $B=0.34$. The parametrization of $R_{T}$ is used to modify nucleon form factors, which leads to the enhancement of QEL structure functions.

Fig. 28 and Fig. 29 show the inclusive neutrino and antineutrino cross sections up to $300 \mathrm{GeV}$ predicted by NuWro. Neutrino cross section shows good agreement with world measurement above $30 \mathrm{GeV}$. While antineutrino cross section is below world measurements in the same neutrino energy range. Contributions from QEL, DIS and SSP are also shown in those figures. The difference between GENIE-Hybrid and NuWro models is shown later in Sec. 8.1. 


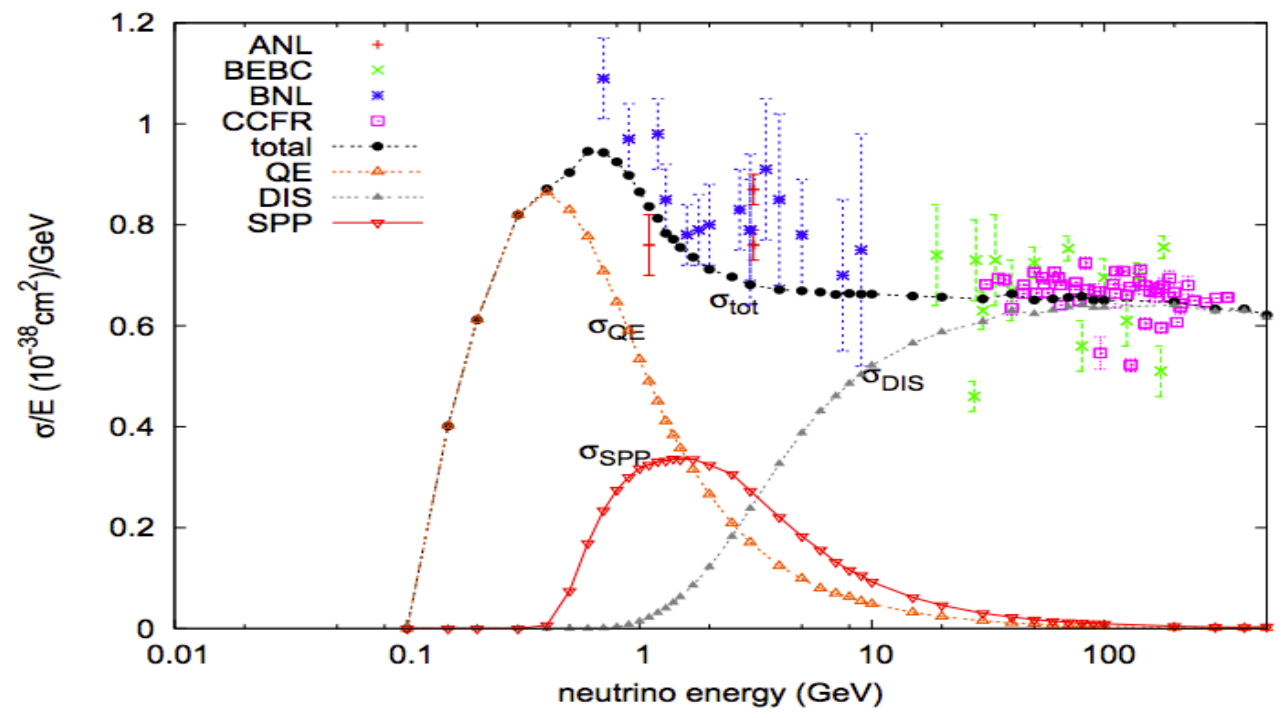

Figure 28: Inclusive neutrino cross section predicted by NuWro, taken from [11]. 


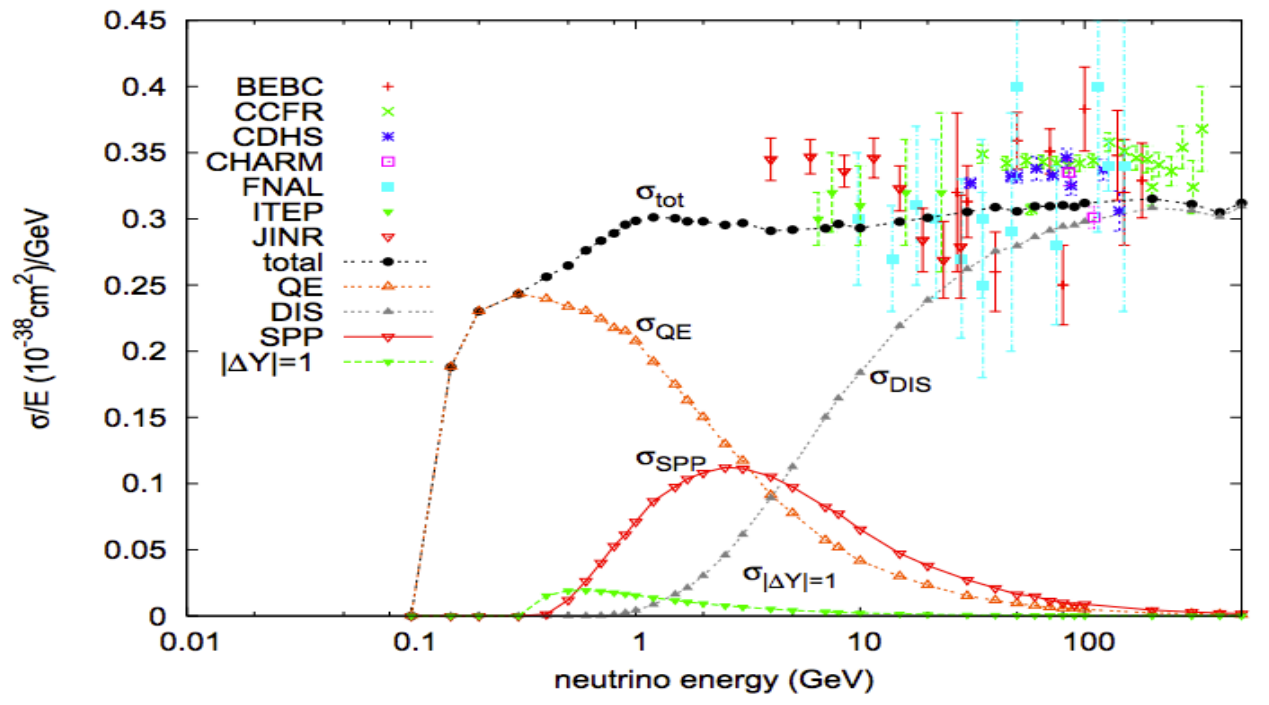

Figure 29: Inclusive antineutrino cross section predicted by NuWro, taken from [11]. 


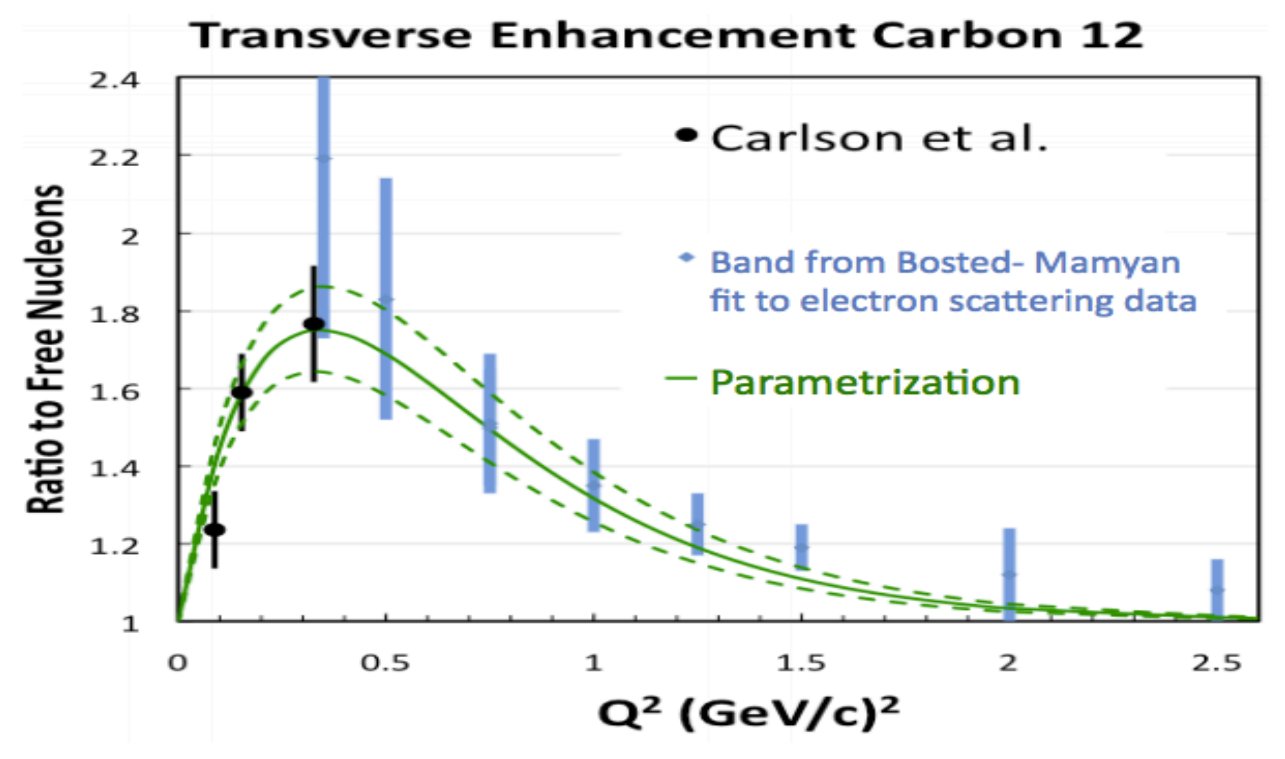

Figure 30: Transverse enhancement ratio as a function of $Q^{2}$, taken from [12]. 


\subsection{RECONSTRUCTION}

Reconstruction of MINER $\nu \mathrm{A}$ neutrino interaction events starts with grouping hits into clusters in time and in space. Groups of clusters are then used to identify tracks of charged particles. In this analysis, we study charged-current muon neutrino interactions which contain a high energy muon. A primary muon track is identified as the longest track which is matched into MINOS ND. Cluster energies which are not associated with the muon tracks form the recoil system, which are summed calorimetrically to obtain the hadronic energy. Fig 31 shows an example of a reconstructed charged-current event. The steps of reconstruction are described in this chapter.

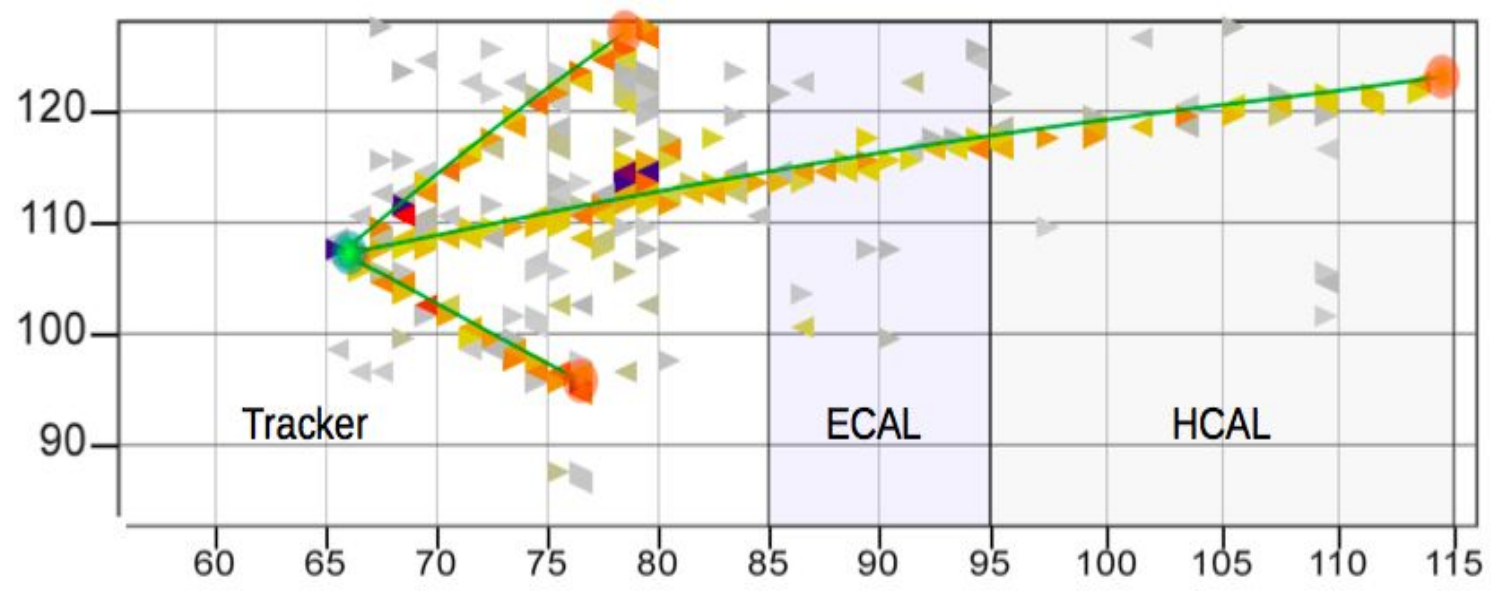

Figure 31: The display of a neutrino interaction event, with a vertex and three tracks reconstructed. 


\subsection{CLUSTER FORMATION}

Due to the high rate beam, multiple events occur within a single NuMI beam spill in the MINER $\nu \mathrm{A}$ detector. Fig. 32 shows an example of a readout gate, of which 7 time slices are identified (with different colors). A time slice is found by scanning the hit time distribution within a spill in blocks of $80 \mathrm{~ns}$. In a scanned block, the photoelectrons are integrated, if it reaches 10 photoelectrons (PE), a new time slice begins. Clusters are formed from groups of hits in a single plane within the same time slice. The time of a cluster is chosen as the hit time for the hit with the most energy.

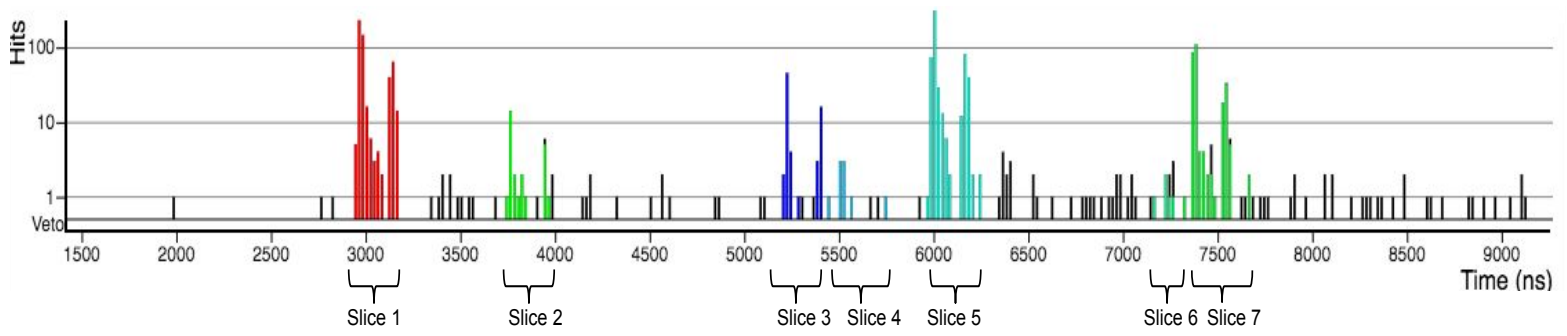

Figure 32: Time slices within the MINERvA Run 2160 Subrun 1 Gate 594. Seven time slices are specified. Black hits are below the energy threshold and not included in energy time slices. (Taken from Arachne [64] page http://minerva05.fnal.gov/Arachne/arachne.html )

The summed energy of all hits forms the "cluster energy". The location of a cluster is calculated from the energy-weighted position of all hits in a cluster. The resulting clusters are classified based on the energy or number of hits: low activity are those with energy less than $1 \mathrm{MeV}$, trackable clusters have total energy between 1 and $12 \mathrm{MeV}$, and have not more than 4 hits, with at least one hit's energy more than $0.5 \mathrm{MeV}$. If more than two hits have hit energy greater than $0.5 \mathrm{MeV}$, they must be next to each other. Heavily ionizing clusters have total energy greater than $12 \mathrm{MeV}$ and the cluster must have at least one hit with energy more than $0.5 \mathrm{MeV}$. Hits with energies more than $0.5 \mathrm{MeV}$ must be adjacent to each other. Superclusters are with energy more than $1 \mathrm{MeV}$ that do not fit the criteria for trackable or heavily ionizing clusters, and any cluster with more than four hits, or cross-talk are made of hits that are correlated with PMT pixels within the cluster. Some clusters are not used in the recoil system reconstruction, based on their classification, which will be described in Sec. 4.5. 


\subsection{TRACK RECONSTRUCTION}

A track is reconstructed to approximate a charged particle's trajectory in the detector. The LongTracker pattern recognition creates tracks using trackable and heavy ionizing clusters within a time slice. It starts with forming seeds from trackable and heavy ionizing clusters, which are grouped by $\mathrm{X}, \mathrm{U}$ or $\mathrm{V}$ view. 2-d track candidates formed by track seeds in different views are then combined to a 3-d track candidate. Once identified, tracks are fitted with a Kalman filter [65] routine. The fitted track is then projected into upstream and downstream directions. If there is an unused cluster in the track projection, it is added to the track.

A track cleaning [8] procedure is then applied, which breaks the clusters and removes the associated energy away from the track. We apply a reconstruction correction to improve the muon and recoil energy separation of this "cleaning" algorithm as described in Sec. 4.6.1.

The longest track is chosen as the "anchor track" and its origin is defined as the event vertex. It must be longer than 25 nodes and the origin of the anchor track is defined as the event vertex. The pattern recognition is then repeated with the non-anchor track clusters. If the projection of the track found is less than $100 \mathrm{~mm}$ away from the vertex and its origin is less than $250 \mathrm{~mm}$ from the vertex, the track is kept and it is called "anchored track". This step is repeated and uses fewer clusters each time, and tracks and vertices are fitted after each pattern recognition. More details of track reconstruction can be found in [8].

\subsection{VERTEX RECONSTRUCTION}

A vertex is reconstructed as the starting point of one or more tracks. The neutrino interaction point is defined as a primary vertex. The position of an interaction vertex is an important criteria in event selection, as described in next chapter.

MINER $\nu$ A uses Kalman filter method [65] for finding the best position of interaction vertex by minimizing the sum of distance between the position of energy deposited and the estimated of track parameters of fitted vertex. This algorithm produces parameters of position, slope, and covariance matrix for each cluster. For two or more tracks with a common interaction vertex, the 
point of closest approach (POCA) of the tracks are taken as the initial vertex position. If there are more than two tracks, a weight is assigned to POCA which is related to the distance between tracks. Tracks with poor compatibility are weighted down in order to improve the reconstruction.

\subsection{CHARGED-CURRENT EVENT RECONSTRUCTION}

A track that begins in the fiducial volume of MINER $\nu \mathrm{A}$, penetrates into MINOS ND and has hits in at least one of the four upstream MINOS planes is considered a "MINOS-matched" muon candidate.

The magnetic field of MINOS ND deflects a charged track. The charge-sign of each MINOSmatched track is determined by the direction of curvature of deflection. The reconstructed track momentum includes the amount of energy lost in the material traversed within MINER $\nu \mathrm{A}$ and the momentum reconstructed in MINOS ND. MINOS ND uses two methods to reconstruct muon

momentum: by range or by curvature, which has been described in Sec. 2.3. Reconstruction by range is more precise and is used for lower energy. If the muon is energetic enough to escape from the calorimeter region of MINOS ND, its momentum is reconstructed by curvature. If it passes through the coil hole region, the tracking algorithm may fail and curvatures as well as momentum will be poorly reconstructed. We removed these events from our analysis by applying a coil hole cut, which is described in Chapter 5.

\subsection{RECOIL SYSTEM RECONSTRUCTION}

The recoil system is constructed from the sum of all the clusters that were not included in the MINOS-matched muon track. The component clusters are required to be within a time window $[20,+35]$ ns of the event time and not to be identified as low activity(less than $1 \mathrm{MeV}$ ) or cross talk. The energy value of the recoil is computed calorimetrically [8], it is defined as

$$
E_{\text {had }}=\alpha \times \sum_{i} C_{i} E_{i}
$$


where $\alpha$ is an overall scale factor, $C_{i}$ is the calorimetric constant for subdetector $i$ and $E_{i}$ is the total visible recoil energy in subdetector $i$, with $i=$ Tracker, Ecal, Hcal.

The calorimetric constants, $C_{i}$, are calculated as

$$
C_{i}=\frac{E_{a b s}+E_{s c i n t}}{f \times E_{s c i n t}}
$$

where $E_{a b s}$ is the energy lost in a single plane of absorber and $E_{\text {scint }}$ is the energy lost in a scintillator plane respectively of a minimum ionizing particle traveling perpendicular to the plane. $f$ is the active fraction $(\mathrm{CH})$ of the scintillator plane [66]. For tracker, $E_{a b s}=0$ and $f=81.85 \%$, which gives $C_{\text {tracker }}=1.22$ as the constant. The constants measured for ECAL and HCAL are $C_{E C A L}=2.013$ and $C_{H C A L}=10.314$, respectively. The overall scale factor, $\alpha$, is set to 1.51 for neutrino and 1.54 for antineutrino, to make the reconstructed recoil energy match the true recoil energy.

In addition, the calorimetric summed recoil energy is corrected with a polyline. This polyline correction is derived from the quantity $\frac{E_{h a d}^{\text {reco }}-E_{h a d}^{\text {true }}}{E_{\text {had }}^{\text {true }}}$ in bins of $E_{\text {had }}^{\text {true }}$.

\subsection{RECONSTRUCTED EVENT CORRECTIONS}

Reconstruction corrections are applied to showers and track vertex before we select a event sample for this analysis. "Shower cleaning corrections" improve the separation of reconstructed hadron energy and muon energy. "Calorimetry fix" removes the effect of PMTs with problematic behaviors. "Vertex correction" removes the events originated outside the fiducial region. In addition tracking efficiency corrections, which account for inefficiency in MINOS-matched tracks and are only applied to Monte Carlo sample, will be discussed later in Sec. 8.3.

\subsubsection{Shower Cleaning Corrections}

"MINOS-matched" rock muon samples (as described in Sec. 2.1) are used to study the distribution of "muon fuzz" (delta or bremsstrahlung) energy. 


\begin{tabular}{|l|l|}
\hline Correction & Effect \\
\hline Shower cleaning corrections & Change hadronic energy and muon energy distribution \\
Calorimetry fix & Removes bad PMTs in minerva1 sample, change hadronic energy \\
Vertex correction & $\begin{array}{l}\text { Removes events with vertices outside fiducial region } \\
\text { and changes hadronic energy }\end{array}$ \\
\hline
\end{tabular}

Table 9: Reconstructed event corrections.

We define the energy sum of all non-muon track clusters found inside subdetector $i$ as $E_{i}^{s h, R}$ (assuming the inner detector is a cylinder with radius $R=1000 \mathrm{~mm}$ ). This can be written as

$$
E_{i}^{s h, R}=\Sigma_{j} E_{i, j}^{s h, R}
$$

where $j$ stands for the cluster ID based on truth information (for example, a proton, pion, neutron, muon, cross talk, etc.) Similar as Eq. 4.1, $E_{i}^{s h, R}$ is a calorimetrical sum of cluster energies.

For the case of the rock muon sample, which has no true hadronic shower, $E_{i}^{s h, R}$ is from the muon track (muon fuzz), $E_{i}^{s h, r=1000 m m}=E_{i, \text { muon }}^{s h}$. We obtain $E_{i}^{s h}$ (per mm) $\delta E_{i}^{s h}$ from

$$
\delta E_{i}^{s h}=\frac{E_{i}^{s h, R}}{D_{i} / \cos \theta_{\mu}}
$$

where $D_{i}$ is the $z$ distance between the starting point and end point of muon track in subdetector $i$ (in $\mathrm{mm}$ ). $\theta_{\mu}$ is the muon track angle with respect to (w.r.t.) the beam direction.

As shown in Fig. 33, there is more $\Delta E_{i}^{s h}$ in data than in MC. The MC at the simulation stage does not generate enough "muon fuzz". In a charged-current event, this "extra" true muon energy is associated with the hadron shower system. In order to better determine muon track energy, $E_{\mu}^{\text {track}}$, and hadron energy, $E_{\text {had }}$, in a charged-current event, as discussed below, we introduce two energy corrections (based on region), which are then subtracted from hadron energy $E_{\text {had }}$ and added into muon track energy $E_{\mu}^{\text {track }}$ for both data and MC. 

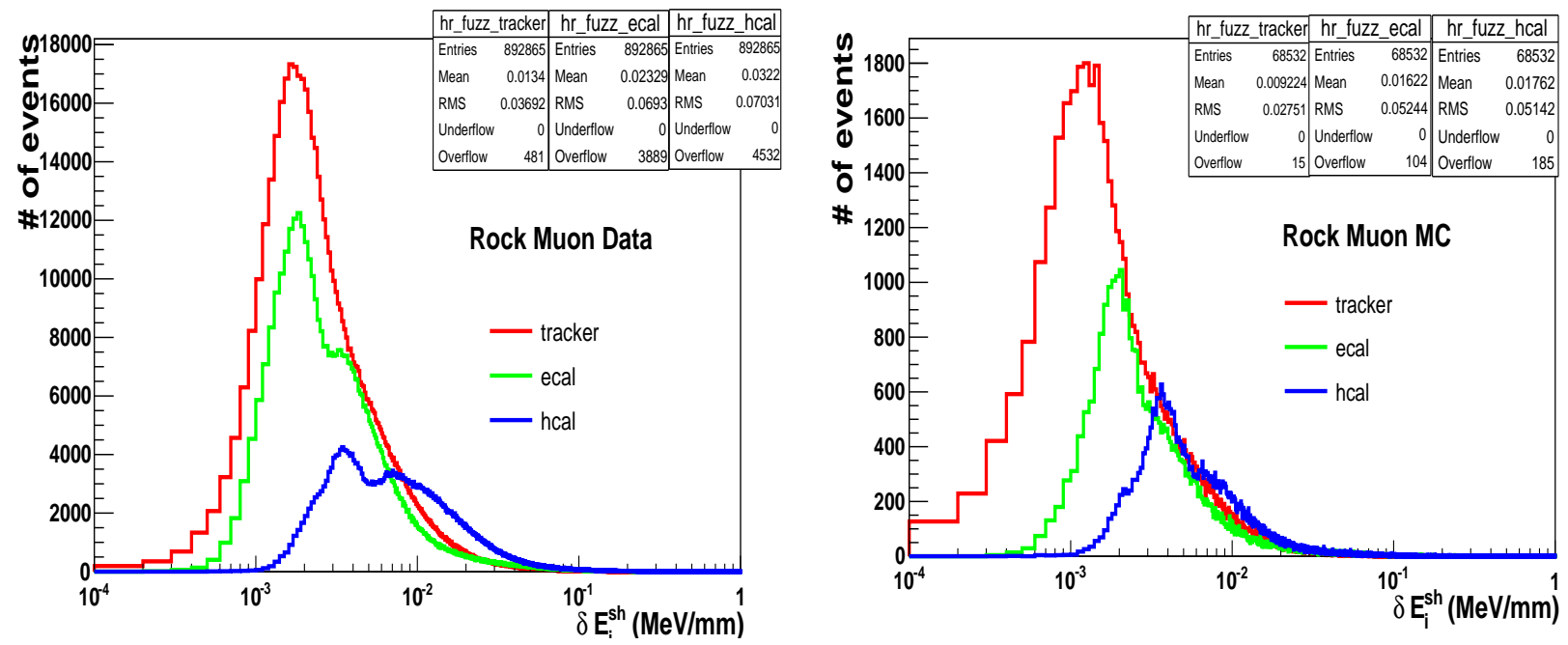

Figure 33: $\delta E_{i}^{s h}$ distributions in subdetector $i$ ( $i=$ tracker, ecal, or hcal) for rock muon sample of data(left) and MC(right).

4.6.1.1 Cylinder Region Fig. 34 shows an example of a charged current event. The black line with the arrow represents a muon track. We define a cylinder along the muon track, which starts at a distance $d=300 \mathrm{~mm}$, from the vertex of muon track. We allow it to have different radii in different regions of the detector, for example, radius in sub-detector $i$ ( $i=$ tracker, ecal or hcal) is defined as $r_{i}\left(r_{\text {tracker }}=80 \mathrm{~mm}, r_{\text {ecal }}=100 \mathrm{~mm}\right.$, and $\left.r_{\text {hcal }}=100 \mathrm{~mm}\right)$.

Sum of all non-muon track energies found inside a cylinder with radius, $r_{i}$, is defined as

$$
E_{i}^{s h, r_{i}}=\Sigma_{j} E_{i, j}^{s h, r_{i}}
$$

Muon purity, $F_{i}$, is defined as the fraction of true muon energy out of all cluster energy found inside the cylinder

$$
F_{i}=\frac{E_{i, m u o n}^{s h, r_{i}}}{E_{i}^{s h, r_{i}}}
$$

in which true cross talk is removed from the denominator. Muon purities for different $\nu$ values are obtained using separate MC samples for neutrino and antineutrino charged-current events. The given results are shown in Tab. 10. Neutrino and antineutrino samples have the similar size of muon 


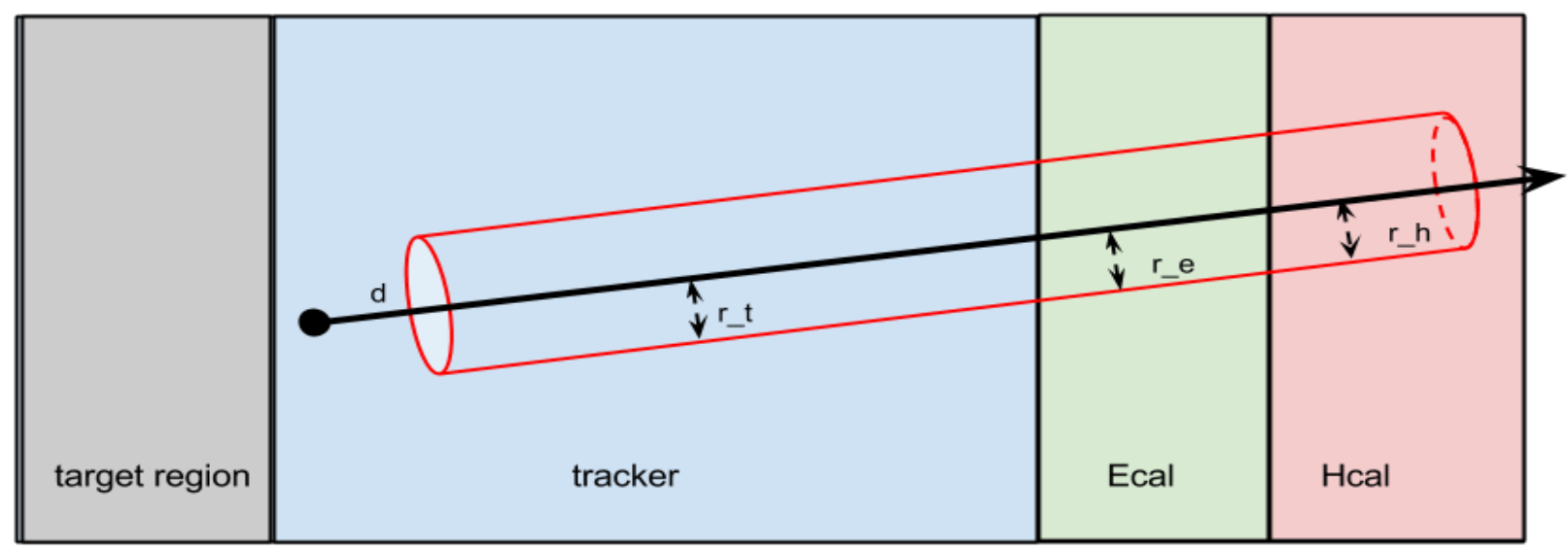

Figure 34: An example of a muon track starting in the tracker. A cylinder along the track is defined. It starts at a distance, $d$, away from the vertex, and has different radii in different regions of the inner detector.

purity in each hadron energy bin, while the difference in the hadron energy distribution between neutrino and antineutrino makes the overall muon purity different.

An energy correction, $E_{i n}^{s h}$, is defined inside the cylinder as

$$
E_{i n}^{s h}=\Sigma_{i} E_{i}^{s h} F_{i}
$$

In order to avoid negative hadron energies, if $E_{i n}^{s h} \geq E_{\text {had }}$, it is set to zero.

4.6.1.2 Non-cylinder Region Another energy correction is needed to remove muon related energy that is deposited outside the cylinder region.

First, we define muon completeness, $T_{i}$, as

$$
T_{i}=\frac{E_{i, \text { muon }}^{s h, r_{i}}}{E_{i, \text { muon }}^{\text {sh,R }}},
$$

which is related to how much of the muon energy leaks out of the cylinder with radius $r_{i}$. For simplicity, the rock muon sample is used to study the muon completeness, since $E_{i, \text { muon }}^{s h, r_{i}}=E^{s h, r_{i}}$. 
Muon completeness, $T_{i}$ 's, are found to be $57 \%, 100 \%$ and $95 \%$ for tracker, ecal and hcal, respectively.

For each muon track of a reconstructed charged-current event, we randomly pick a rock muon track from the distribution of $\delta E_{i}^{s h}$ from the rock muon sample and obtain a correction

$$
E_{\text {out }}^{\text {sh }}=\Sigma_{i} \delta E_{i}^{s h} L_{i}\left(1-T_{i}\right)
$$

where $\delta E_{i}^{s h}$ is defined in Eq. 4.4. which is different between data and MC as shown in Fig. 33. $L_{i}$ is the track length of the muon track in subdetector $i$ in an inclusive event, which is defined as

$$
L_{i}=\frac{D_{i}}{\cos \theta_{\mu}} .
$$

If $E_{\text {out }}^{\text {sh }} \geq E_{\text {had }}$, we will randomly pick another rock muon, until $E_{\text {out }}^{f u z z}<E_{\text {had }}$. The corrected hadron energy is

$$
E_{\text {had }}^{\text {new }}=E_{\text {had }}-E_{\text {in }}^{\text {sh }}-E_{\text {out }}^{\text {sh }},
$$

while the corrected muon energy becomes

$$
E_{\mu}^{\text {new }}=E_{\mu}^{\text {track }}+E_{\text {in }}^{s h}+E_{\text {out }}^{\text {sh }}
$$

We refer to effect of $E_{\text {had }}-E_{\text {had }}^{\text {new }}$ and $E_{\mu}^{\text {new }}-E_{\mu}^{\text {track }}$ as the shower cleaning correction.

Fig. 35 shows the effect of this "shower cleaning correction" on the distribution of hadron energy within neutrino energy bin 2-3 GeV for data and MC. For both data and MC, events migrate to lower hadron energy bins after applying shower cleaning correction.

\subsubsection{Calorimetry Fix}

Two PMTs, which are at module 103 (strip:65-96) and module 105 (strip:65-96), in the HCAL were found to have high un-simulated cross-talk [67]. For minerva1 data and simulated Monte Carlo (MC) samples, cluster energy (which are not identified as cross talk or low activity cluster energy and within $[-20,+35]$ ns time window) measured using these two PMTs are removed from the hadronic energy. This is not a problem for later run periods minerva5 and minerva13C, before which the problem was fixed. 


\subsubsection{Vertex Correction}

A correction is applied to correct the vertex position of events whose vertices are poorly reconstructed due to the presence of an energetic hadron shower [67]. We search for visible activity in a cone upstream of the muon track and move the vertex location upstream along the muon trajectory. The cone is defined as one scintillator strip wide at the module of the reconstructed vertex and expands by one strip for every additional upstream module. A $1.5 \mathrm{MeV}$ threshold cluster energy is required to shift the vertex(one module wide gaps are permitted). The approximate energy deposition of a muon traversing a plane is removed from the recoil system for every plane the vertex is shifted. Events whose vertex is moved out of fiducial volume after this correction are removed from the sample. 


\begin{tabular}{|c|ccc|ccc|}
\hline & \multicolumn{3}{|c|}{ neutrino } & \multicolumn{3}{c|}{ antineutrino } \\
\hline subdetector & tracker & ecal & hcal & tracker & ecal & hcal \\
\hline$\nu<0.3 \mathrm{GeV}$ & $97.7 \%$ & $99.6 \%$ & $99.9 \%$ & $97.3 \%$ & $99.4 \%$ & $99.9 \%$ \\
$0.3<\nu<0.5 \mathrm{GeV}$ & $91.7 \%$ & $98.0 \%$ & $99.8 \%$ & $87.3 \%$ & $96.7 \%$ & $99.4 \%$ \\
$0.5<\nu<1 \mathrm{GeV}$ & $79.3 \%$ & $93.7 \%$ & $99.1 \%$ & $74.8 \%$ & $91.1 \%$ & $98.6 \%$ \\
$1<\nu<2 \mathrm{GeV}$ & $60 \%$ & $82.6 \%$ & $96.5 \%$ & $58 \%$ & $81 \%$ & $95.9 \%$ \\
$2<\nu<22 \mathrm{GeV}$ & $27.1 \%$ & $46.9 \%$ & $74.9 \%$ & $27.3 \%$ & $50.7 \%$ & $79.3 \%$ \\
\hline Average $(\nu<22 \mathrm{GeV})$ & $54.8 \%$ & $73.3 \%$ & $89.0 \%$ & $63.3 \%$ & $80.9 \%$ & $93.6 \%$ \\
\hline
\end{tabular}

Table 10: Muon purity used in the cylinder correction. Corrections are applied in bins of hadron energy for $\nu<0.3 \mathrm{GeV}, 0.3<\nu<0.5 \mathrm{GeV}, 0.5<\nu<1 \mathrm{GeV}, 1<\nu<2 \mathrm{GeV}$ and $2<\nu<22 \mathrm{GeV}$, respectively. Bottom row shows the average muon purity for the inclusive sample, these are not used (only for the sake of over all hadron energy region comparison between neutrino and antineutrino). 

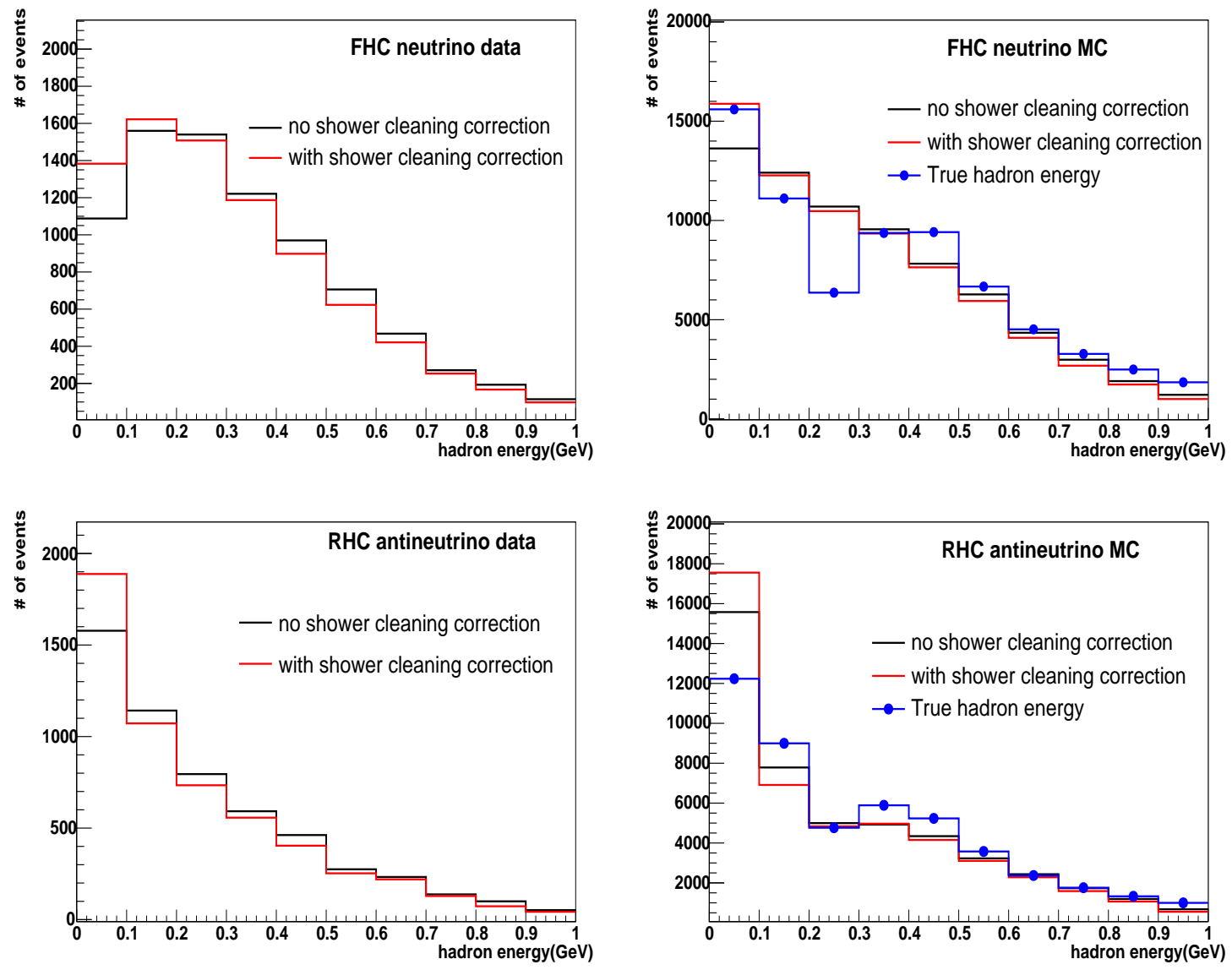

Figure 35: Effect of shower cleaning correction on hadron energy in $E_{\nu}=(2,3) \mathrm{GeV}$ for neutrino (top) and antineutrino (bottom) data and MC. 


\subsection{EVENT SELECTION}

\subsection{DATA SAMPLE}

MINER $\nu \mathrm{A}$ first started collecting data in November 2009, as shown in Figure 36. It completed construction and installation of its modules in the MINOS ND hall in March 2010. In April 2012, MINER $\nu \mathrm{A}$ completed data-taking run with the low-energy(LE) beams for both FHC (neutrinoenhanced) and RHC (antineutrino-enhanced) mode. Medium energy mode started in September 2013.

In this analysis, we are using FHC mode data which corresponds to an exposure of $3.2 \times 10^{20}$ proton on target (POT) and RHC mode data, which corresponds to an exposure of $1.0 \times 10^{20}$ POT. The MC samples used are about ten times the size of data samples.

The event samples needed to extract the cross section and flux are described below. The inclusive sample is related to the total charged current event rate measured in our detector. This sample includes all charged-current events with a muon reconstructed in the MINOS ND. The flux sample is a subset of the inclusive sample used to measure the shape of the incident beam flux with energy.

The selection criteria are:

1. Fiducial volume. Fiducial vertex is defined as a vertex with a hexagonal apothem less than $850 \mathrm{~mm}$ from the axis passing through the center of the detector, which has a minimum of 20 $\mathrm{cm}$ from the edge of active scintillator region and a $z$ position larger than 5990mm and less than $8340 \mathrm{~mm}$, which excludes the first 8 planes at upstream and the last 12 planes at downstream of active scintillator region.

2. Charged current selection. We require a MINOS-matched track, a reconstructed track exiting from the back of MINER $\nu \mathrm{A}$, matching to a reconstructed track in MINOS, with negative 


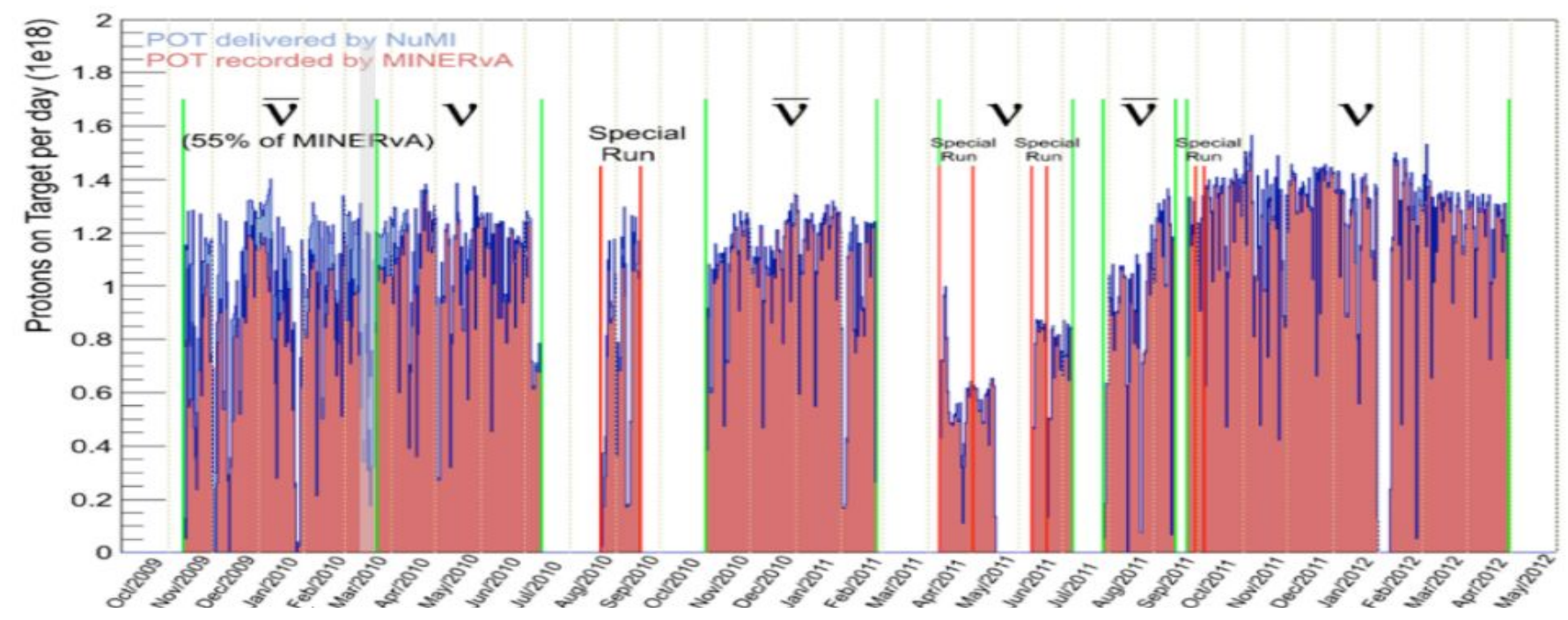

Figure 36: MINER $\nu$ A Low Energy data starting from November 2009.

(positive) curvature for neutrino (antineutrino) and a reconstructed momentum in MINOS.

3. Track quality. To remove tracks with large error on $q / p$ measurement, we require tracks to have an error on measured charge to momentum ratio $(q / p)$ to be less than $30 \%$. It removes about $9 \%$ events in MC sample and reduces the wrong-sign contamination by $20 \%$ at higher neutrino energy region for MINOS upstream sample, which is defined in the next section.

4. Kinematic cuts. We require a minimum energy of $1.8 \mathrm{GeV}$ and a maximum muon angle w.r.t beam of 0.35 rads $(20 \mathrm{deg})$ for reconstructed muon track. It improves the overall sample acceptance in the lowest energy bins and allows us to compute an explicit model-based kinematic acceptance correction, which will be discussed in detail later in next chapter.

5. Coil hole removal. We remove those tracks which end on the left and $80 \mathrm{~cm}$ away from MINOS coil hole center, in which region the wrong-sign contamination of MINOS upstream sample is up to about $50 \%$ at high energy. This cut removes those events which are poorly reconstructed due to the coil hole, therefore it reduces the wrong-sign contamination in the selected samples. About 2.2\% events is removed due to this cut for FHC neutrino data and simulated samples.

6. Dead time cut. We require the number of dead discriminator pairs in the (primary) track upstream projection to be zero or 1 , since readout electronics have $200 \mathrm{~ns}$ dead time after each 
recording, which would cause failures of event reconstruction coming later in time.

MC simulation shown in this section uses the samples with hadron production model flux, GENIEHybrid model and are data overlaid.

Figure 37 shows the effect of each cut applied step by step for FHC neutrino sample. The black points shows the the ratio of number of events passing fiducial and MINOS-Matched cuts to the number of events with no cuts, in each reconstructed neutrino energy bin. The fraction increases with neutrino energy up to $60 \%$ at $20 \mathrm{GeV}$. The other color show the ratio with one additional cut applied in each case. The coil hole and dead time cuts have only a tiny effect comparing with others. Muon energy and muon angle cut have the largest effect at low energy. Fig. 38 shows a comparison of the reconstructed energy spectrum of FHC neutrino sample with each applied cut. Tab. 11 shows the number of events left after applying each cut for FHC neutrino and RHC antineutrino data and simulated samples.

\begin{tabular}{|c|cc|cc|cc|cc|}
\hline \hline selection & $\begin{array}{c}\text { neutrino } \\
\text { data }\end{array}$ & & $\begin{array}{c}\text { neutrino } \\
\text { MC }\end{array}$ & & $\begin{array}{c}\text { antineutrino } \\
\text { data }\end{array}$ & $\%$ & $\begin{array}{c}\text { antineutrino } \\
\%\end{array}$ & MC \\
\hline fiducial events & 285153 & $100 \%$ & 1957050 & $100 \%$ & 167397 & $100 \%$ & 1118470 & $100 \%$ \\
MINOSMatched & 134173 & $47.1 \%$ & 1036170 & $52.9 \%$ & 92764 & $55.4 \%$ & 731112 & $65.3 \%$ \\
charge selection & 110842 & $38.9 \%$ & 858347 & $43.9 \%$ & 44822 & $26.8 \%$ & 383575 & $34.3 \%$ \\
track quality & 100165 & $35.1 \%$ & 777741 & $39.7 \%$ & 40673 & $24.3 \%$ & 348829 & $31.2 \%$ \\
coil hole cut & 97932 & $34.3 \%$ & 759198 & $38.8 \%$ & 40062 & $23.9 \%$ & 343615 & $30.7 \%$ \\
dead time cut & 96700 & $33.9 \%$ & 752714 & $38.5 \%$ & 39780 & $23.8 \%$ & 341869 & $30.6 \%$ \\
kinematic cuts & 94086 & $33.0 \%$ & 727923 & $37.2 \%$ & 38994 & $23.3 \%$ & 334276 & $29.9 \%$ \\
\hline \hline
\end{tabular}

Table 11: Effect of event selection cuts for both FHC (minerva13C only) and RHC data and simulated samples. The second column in each section shows the fraction remaining after each cut. Hadron production flux described in Sec. 2.1 is used for MC.

Figure 39 shows the effect of each cut applied step by step for RHC antineutrino sample. The fiducial cut and MINOS-matched cut show similar size of effect as FHC neutrino. However, the charge cut removes more than $30 \%$ of events above $7 \mathrm{GeV}$, which is due to a much larger fraction 

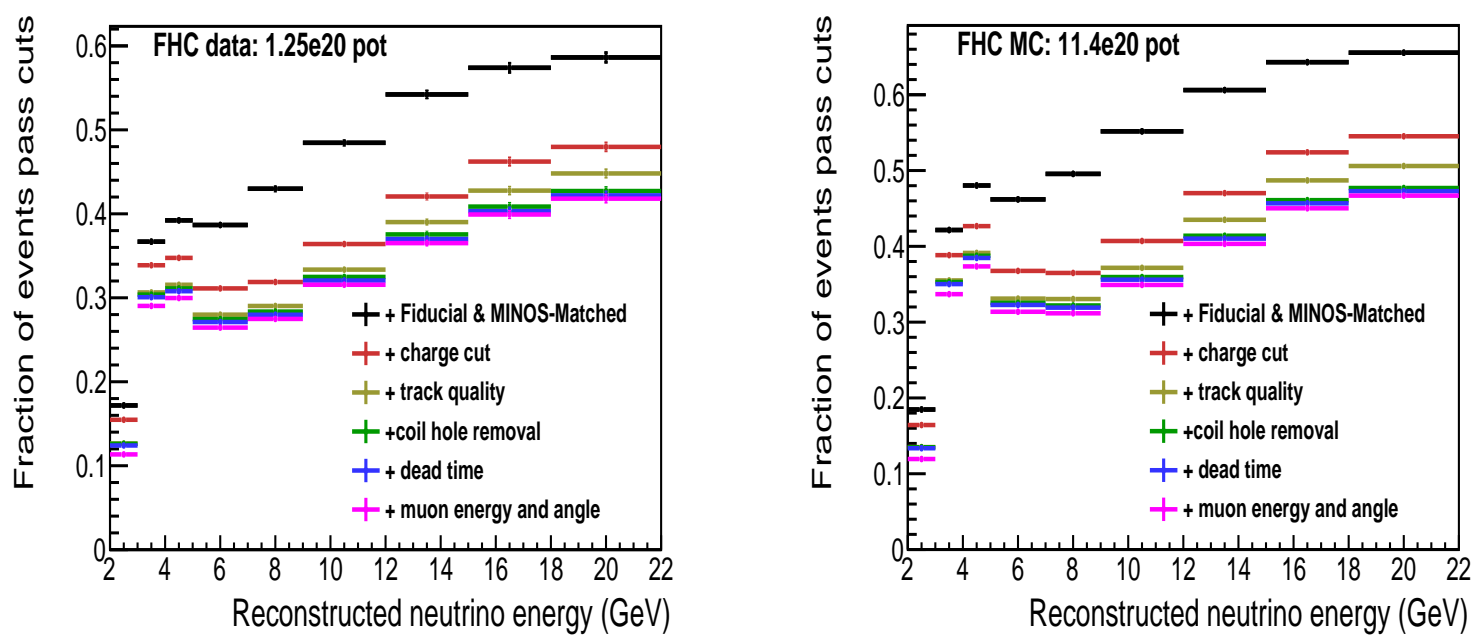

Figure 37: Cut efficiency as a function of reconstructed neutrino energy for FHC neutrino data (left) and simulated (right) samples. Hadron production flux described in Sec. 2.1 is used for MC.

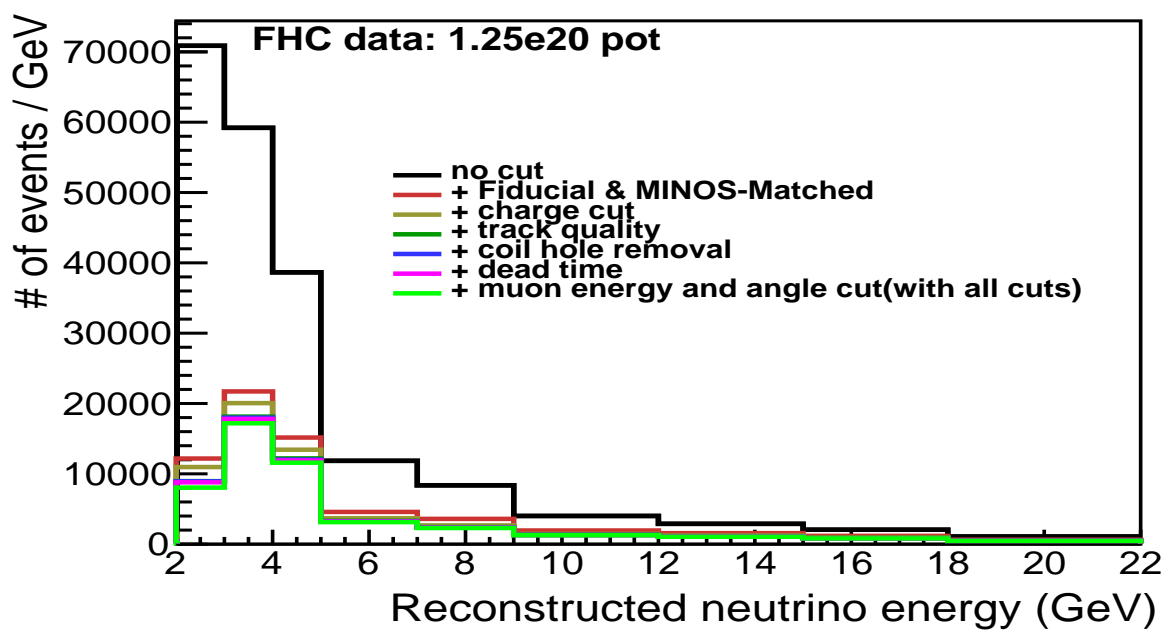

Figure 38: Inclusive Sample of FHC neutrinos with and without all cuts. (39\% of FHC data is used for this figure.) 
of neutrino contamination in the RHC antineutrino beam. Fig. 40 shows a comparison of RHC antineutrino sample with and without any cuts.
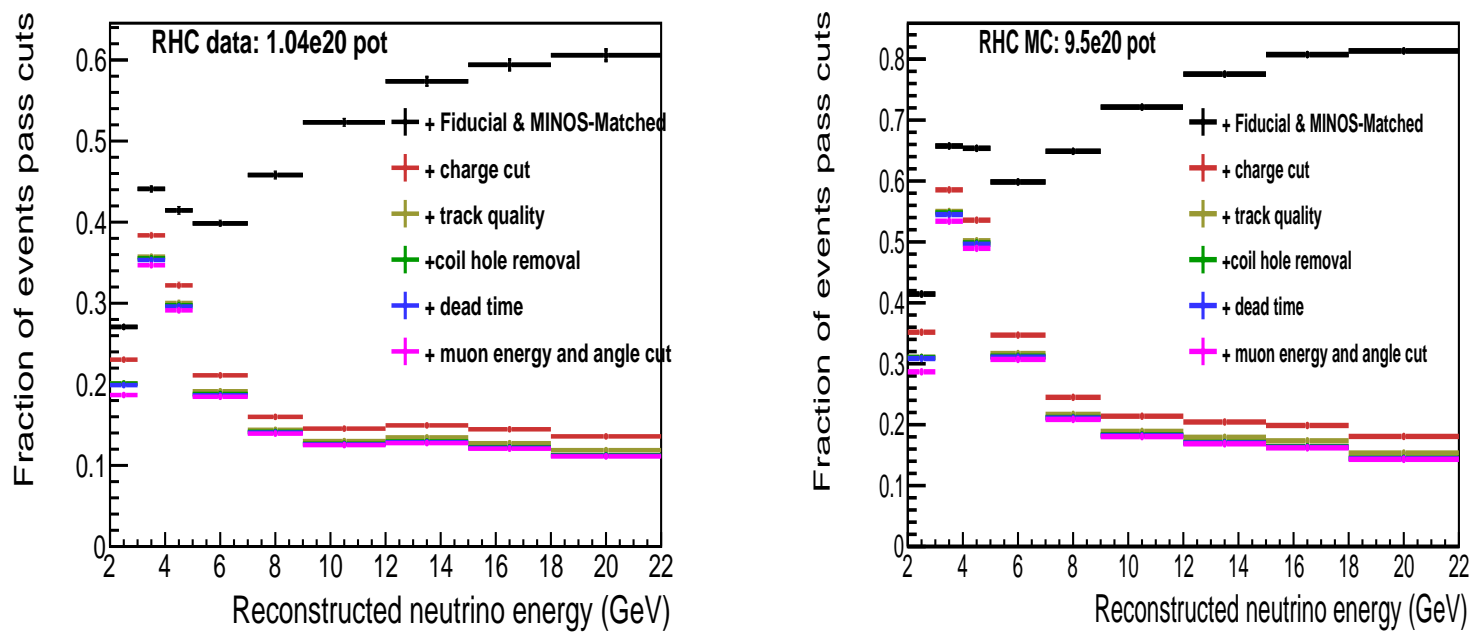

Figure 39: Cut efficiency as a function of reconstructed neutrino energy for RHC antineutrino data (left) and simulated samples.

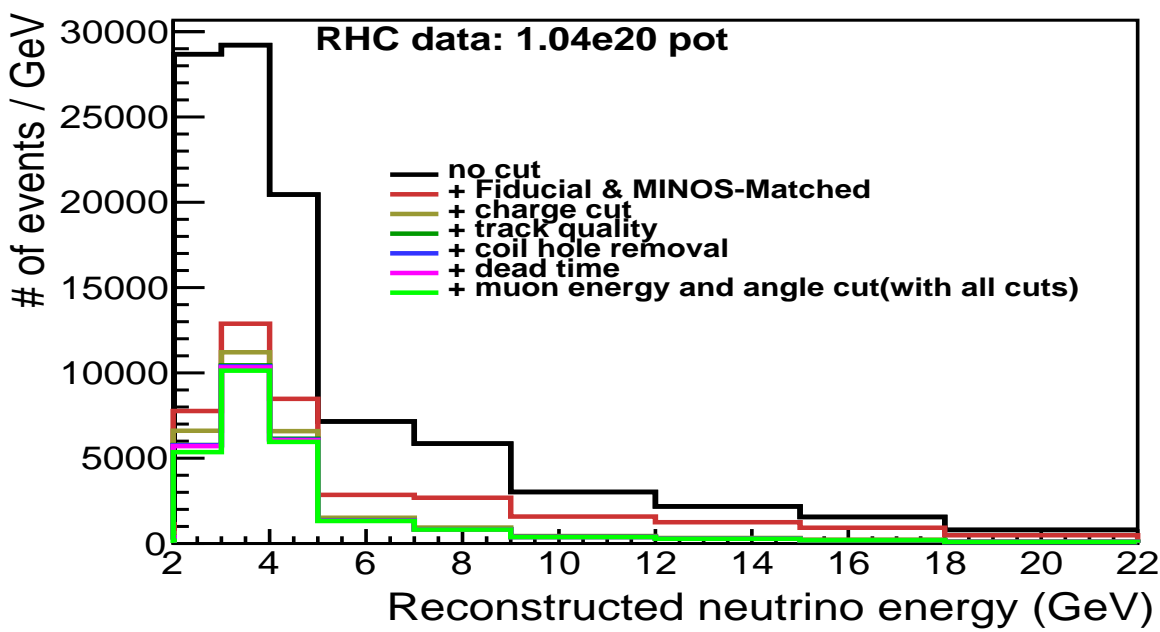

Figure 40: Inclusive Sample of RHC neutrinos with and without all cuts.

The raw flux event sample in a given energy bin is defined as the number of CC-inclusive events in the bin with a hadron energy cut $\nu<\nu_{o}$. The same selections are applied to this sample 
with one additional requirement on the maximum $\nu$ value. Table 12 shows the value of $\nu_{o}$ for each energy bin.

The selected inclusive and flux samples are shown in Fig. 41. The ratios of selected flux samples to inclusive sample are shown in Fig. 42. The $\nu$ cut is increased with neutrino energy to improve flux sample statistical precision.
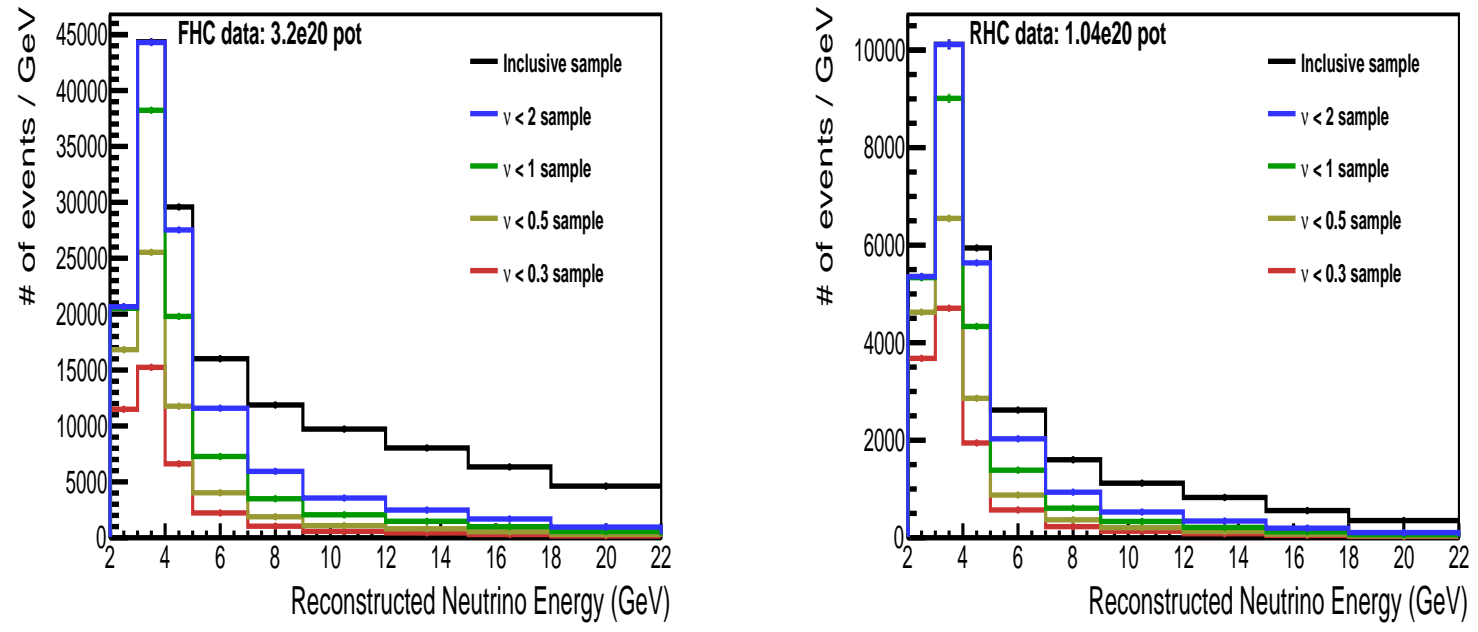

Figure 41: Cross section and flux samples for FHC neutrino(left) and RHC antineutrino(right). 

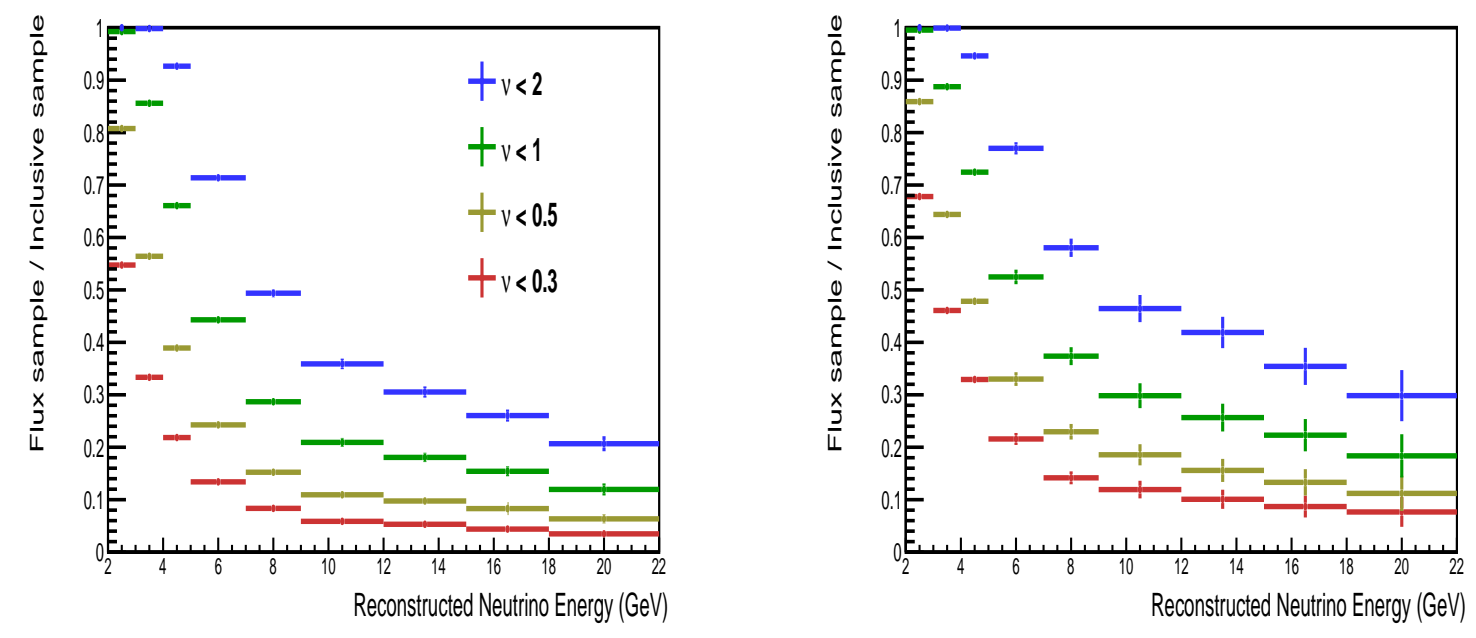

Figure 42: Ratio of flux samples to inclusive sample for FHC neutrino(left) and RHC antineutrino(right).

\begin{tabular}{|c|c|c|}
\hline \hline Energy range $(\mathrm{GeV})$ & Energy bin $(\mathrm{GeV})$ & $\nu_{0}(\mathrm{GeV})$ \\
\hline $2-3$ & $2-3$ & 0.3 \\
\hline $3-7$ & $3-4$ & \\
& $4-5$ & 0.5 \\
& $5-7$ & \\
\hline $7-12$ & $7-9$ & 1.0 \\
& $9-12$ & \\
\hline $12-22$ & $12-15$ & \\
& $15-18$ & 2.0 \\
& $18-22$ & \\
\hline
\end{tabular}

Table 12: Value of $\nu_{o}$ for each energy range. 


\subsection{MINOS SUBSAMPLE STUDY}

Muon tracks ending in different regions of MINOS ND are reconstructed differently (i.e. the momentum of tracks end in the downstream part of MINOS ND are always reconstructed by curvature, while for those ends in the upstream part, both range and curvature are used). In order to understand the acceptance correction (detector smearing effect and bin migrations) of MINOS, the selected samples is divided into four categories based on where the muon track ends in MINOS ND.

Four MINOS subsamples are defined if tracks exiting MINERVA and entering MINOS

- Upstream Stopping: Contained in MINOS upstream calorimeter region,

- Downstream Stopping: Contained in MINOS downstream spectrometer region,

- Upstream Exiting: Exiting the MINOS upstream calorimeter region,

- Downstream Exiting: Exiting the MINOS downstream calorimeter region.

The muon momentum for both two downstream samples are reconstruction by curvature. However, we still separate those two samples in order to be consistent with MINOS's definition.

Fig. 43 shows the muon track end distribution in MINOS ND for each subsample. Events that with tracks stopping at a distance of greater than $50 \mathrm{~cm}$ from the edge of the detector and greater than $80 \mathrm{~cm}$ from the coil hole center are defined as stopping sample. Otherwise, they are defined as exiting sample. Events with muon track exiting from the downstream end of MINOS $\mathrm{ND}$, regardless of $(x, y)$ position, is defined as downstream exiting.

Fig. 44 shows the reconstructed muon energy distribution of selected inclusive sample for FHC neutrinos. The upstream samples dominate in the region $E_{\mu}<6 \mathrm{GeV}$, while the downstream samples dominates above $6 \mathrm{GeV}$ muon energy region. Fig. 45 shows the reconstruction efficiency as a function of reconstructed muon energy for selected inclusive sample for FHC neutrinos. The reconstruction efficiency is defined as the ratio of the number of events reconstructed in an energy bin to the number of events generated in that bin. The overall shape for inclusive sample is given by the sum of the four components. Fig. 46 and Fig. 47 show similar behaviors for inclusive sample of RHC antineutrinos.

The distributions of MINOS subsamples as a function of reconstructed muon energy are inde- 
pendent of the $\nu$ cut. The overall reconstruction efficiency increases as $\nu$ cut decreases for both FHC neutrinos and RHC antineutrinos. It is about 70\% (80\%) at high energy for FHC (RHC) inclusive sample, which it is $90 \%$ (90\%) for $\nu<0.3 \mathrm{GeV}$ flux sample.
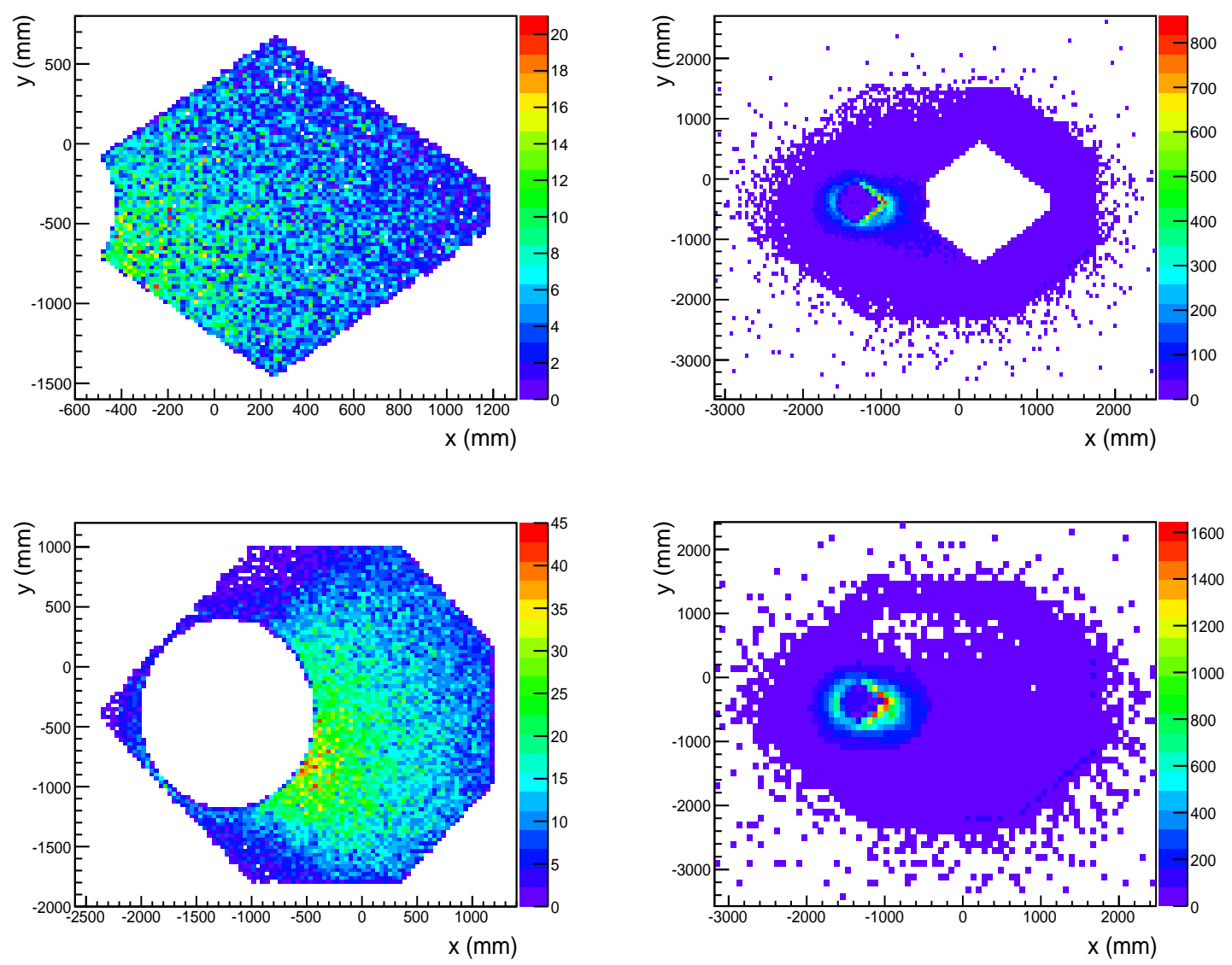

Figure 43: Muon track end distributions of four MINOS subsamples. Upper left: upstream stopping, upper right:upstream exiting (blank region is the same region as in upper left figure), bottom left: downstream stopping (tracks ending within $80 \mathrm{~cm}$ from the coil hole center are excluded), bottom right: downstream exiting. 


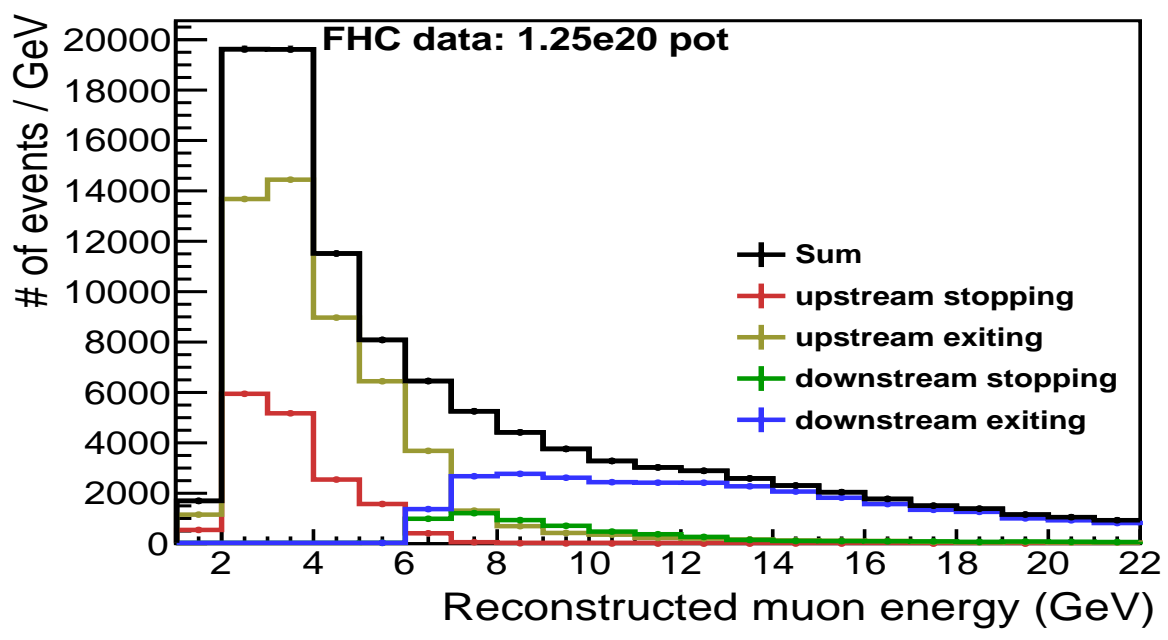

Figure 44: FHC inclusive neutrino sample divided into four subsamples in MINOS ND.

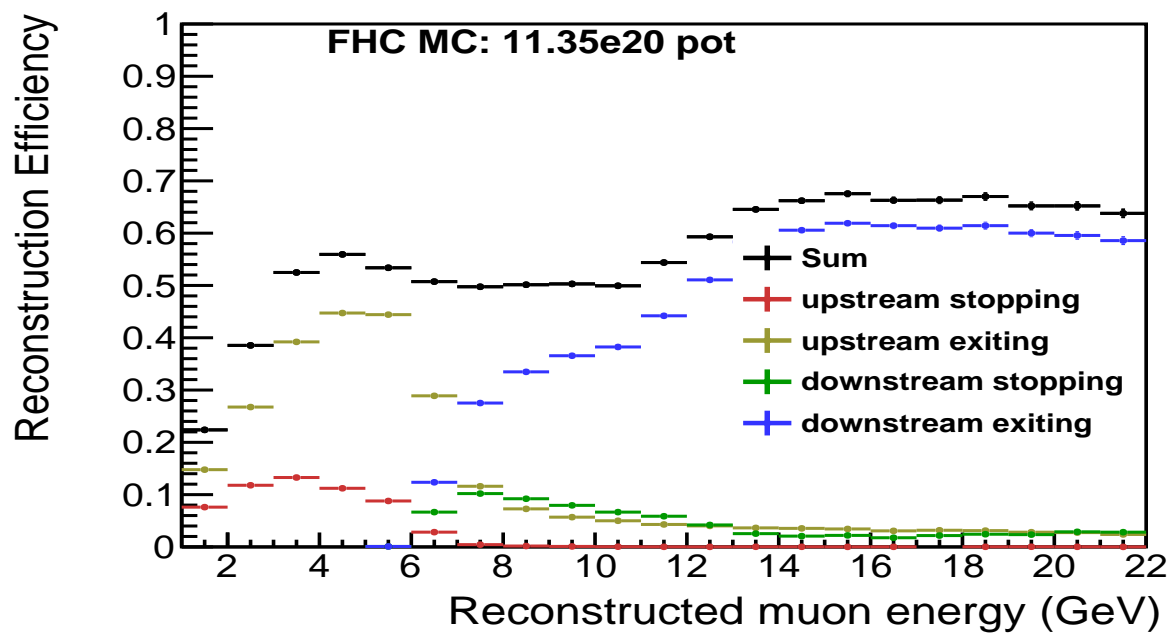

Figure 45: Reconstruction efficiency of different MINOS subsamples for inclusive sample of FHC neutrinos. 


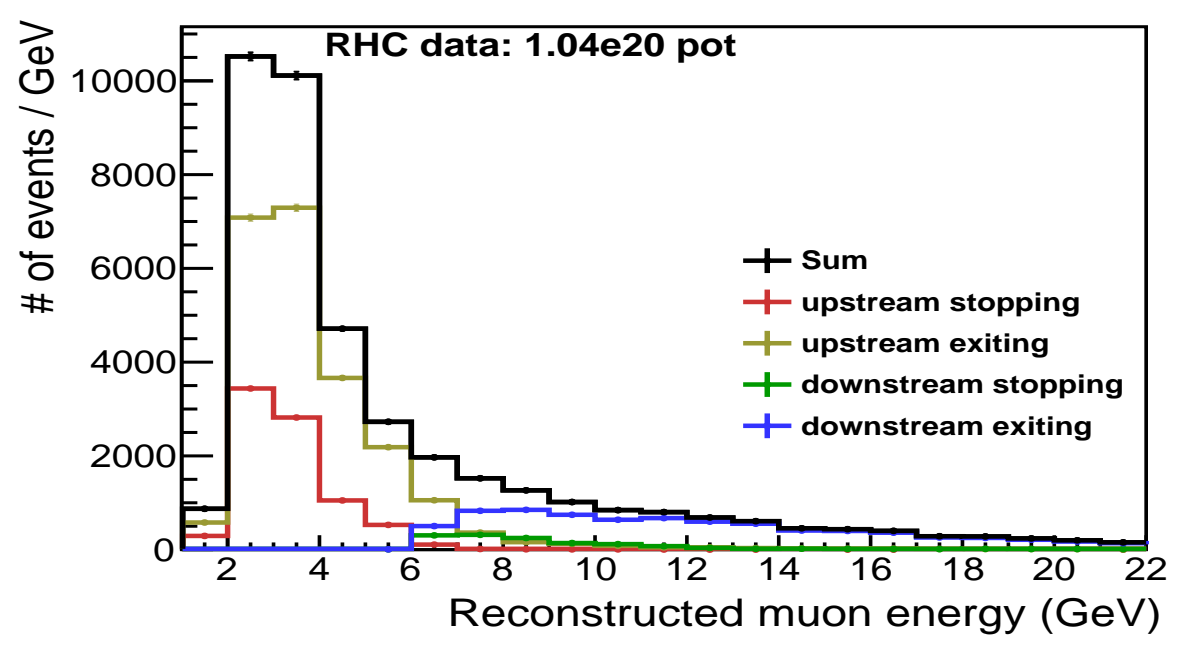

Figure 46: RHC inclusive antineutrino sample divided into four subsamples in MINOS ND.

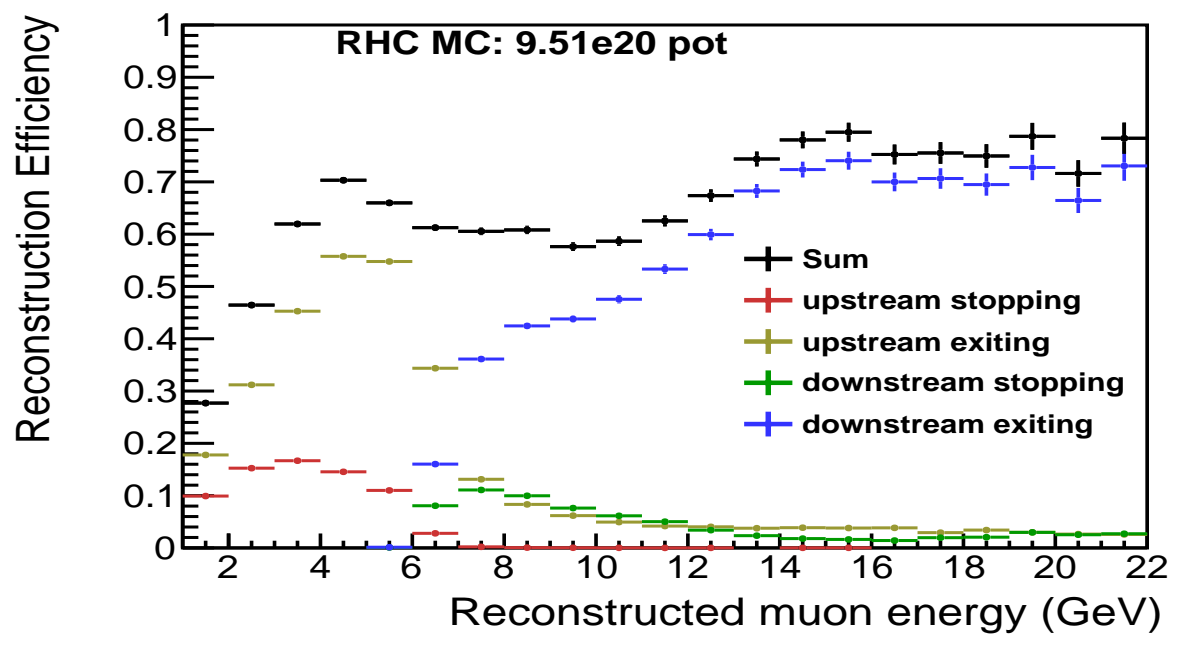

Figure 47: Reconstruction efficiency of different MINOS subsamples for inclusive sample of RHC antineutrinos. 


\subsection{BACKGROUND}

There are two types of backgrounds in the selected samples, wrong-sign contamination and neutralcurrent contamination.

Neutral-current (NC) contamination is from neutral-current events with a long track passing charged-current event sample cuts.

\section{Reconstructed CC \&\& True NC events \\ Reconstructed CC events}

Fig. 48 shows the fractional NC contaminations for neutrino and antineutrino samples. NC contamination is small $(\ll 1 \%)$ for both samples.
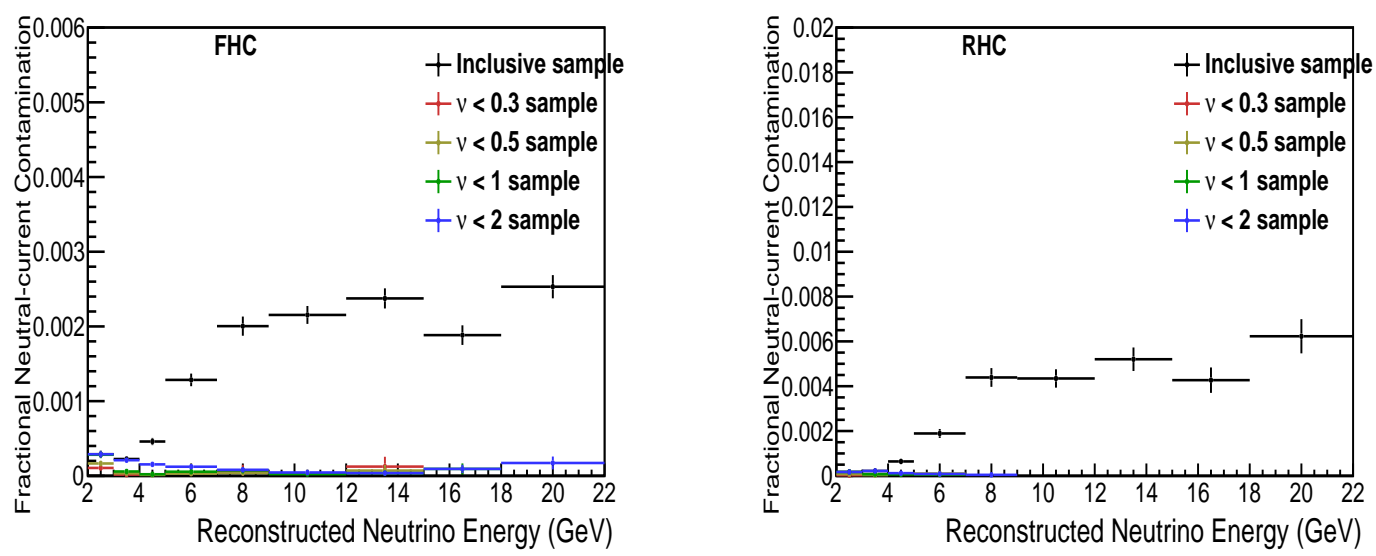

Figure 48: Neutral-current contamination of cross section and flux samples for FHC neutrino (left) and RHC antineutrino (right).

Wrong-sign (WS) contamination is from opposite charge sign events comparing with the generated truth charge sign,

\section{Reconstructed CC \&\& True CC \&\& True WS events Reconstructed CC events}

Fig. 49 shows the fractional wrong-sign contamination for selected neutrino and antineutrino samples. The wrong-sign contamination for FHC neutrino sample is always small $(<1 \%)$. However, wrong-sign contamination for the RHC antineutrino inclusive sample is non-negligible (about 4\% 

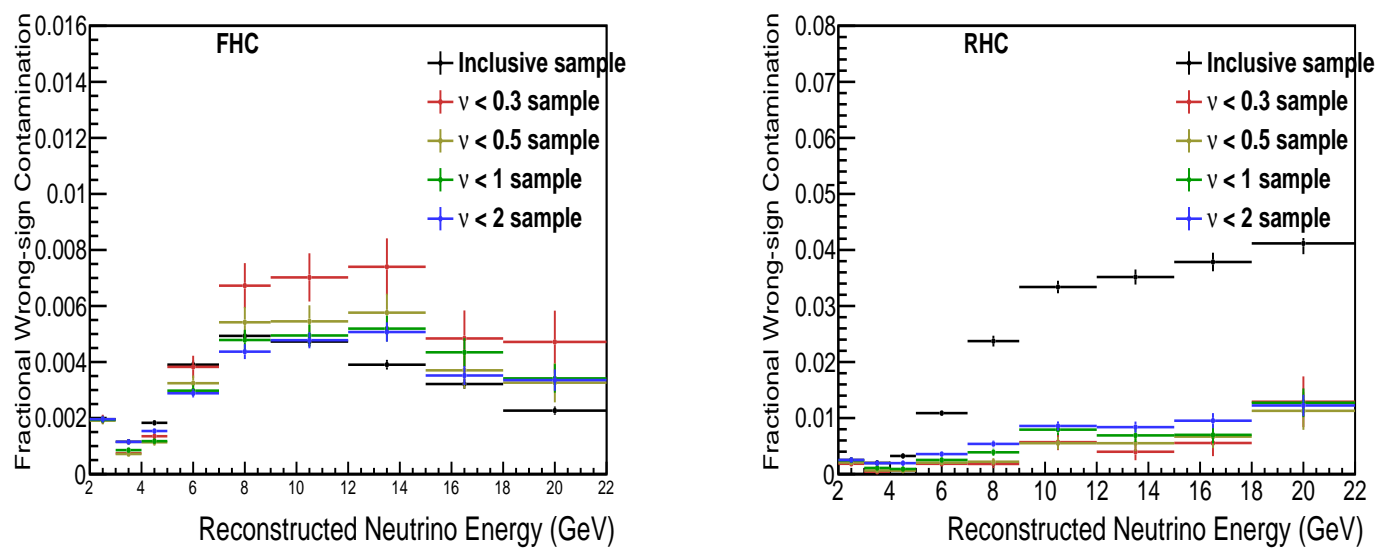

Figure 49: Wrong-sign contamination of cross section and flux samples for FHC neutrino(left) and RHC antineutrino(right).

above $10 \mathrm{GeV}$ ), which is due to a higher $\nu_{\mu}$ component as neutrino energy increasing in RHC beam mode.

The contaminations obtained from MC shown here are subtracted fractionally from data samples, before any correction is applied. Details are presented in Sec. 6.2. 


\subsection{FLUX AND CROSS SECTION EXTRACTION}

In this chapter, we present the method of cross section, the ratio, $r$, and flux measurements. We introduce the low- $\nu$ technique for flux measurement, procedure of cross section extraction, method of data-based normalization, and generator level model-dependent corrections computed.

\subsection{LOW- $\nu$ FLUX MEASUREMENT TECHNIQUE}

The low- $\nu$ method extracts the shape of the flux with energy in neutrino and antineutrino samples. To obtain an absolute flux, an external normalization is needed as discussed below. The method relies on the independence of the low- $\nu$ ( $\nu=$ energy transfer to the hadronic system, which is the hadronic energy, as defined in Sec. 1.3.1) part of the cross section with energy in the limit $\nu \rightarrow 0$. Neutrino and antineutrino scattering off of a target with isospin symmetry are related through the fundamental structure of the charged-current weak interaction. The general form of the cross section in Eq. 1.21 can be re-written in terms of the structure functions and relativistic invariants $\left(x, y, Q^{2}\right)$,

$$
\begin{aligned}
\frac{d^{2} \sigma^{\nu(\bar{\nu})}}{d x d y} & =\frac{G_{F}^{2} M E_{\nu}}{\pi\left(1+Q^{2} / M_{W}^{2}\right)^{2}}\left(\left(1-y-\frac{M x y}{2 E}+\frac{y^{2}}{2} \frac{1+4 M^{2} x^{2} / Q^{2}}{1+R\left(x, Q^{2}\right)}\right) F_{2}^{\nu(\bar{\nu})}\left(x, Q^{2}\right)\right. \\
& \left. \pm y\left(1-\frac{y}{2}\right) x F_{3}^{\nu(\bar{\nu})}\left(x, Q^{2}\right)\right) .
\end{aligned}
$$

The parity violating $F_{3}$ term changes sign for antineutrinos.

The differential dependence of the cross section in terms of $\nu$ can be written as

$$
\frac{d \sigma^{\nu, \bar{\nu}}}{d \nu}=A\left(1+\frac{B^{\nu, \bar{\nu}}}{A} \frac{\nu}{E}-\frac{C^{\nu, \bar{\nu}}}{A} \frac{\nu^{2}}{2 E^{2}}\right)
$$


The coefficients $A, B^{\nu, \bar{\nu}}$, and $C^{\nu, \bar{\nu}}$ depend on integrals over structure functions,

$$
\begin{gathered}
A=\frac{G_{F}^{2} M}{\pi} \int F_{2}(x) d x \\
B^{\nu, \bar{\nu}}=-\frac{G_{F}^{2} M}{\pi} \int\left(F_{2}(x) \mp x F_{3}(x)\right) d x, \\
C^{\nu, \bar{\nu}}=B^{\nu, \bar{\nu}}-\frac{G_{F}^{2} M}{\pi} \int F_{2}(x) \tilde{R} d x,
\end{gathered}
$$

where,

$$
\tilde{R}=\left(\frac{1+\frac{2 M x}{\nu}}{1+R_{L}}-\frac{M x}{\nu}-1\right) .
$$

In the limit of $y=\nu / E \rightarrow 0$ the $B$ and $C$ terms vanish and both cross sections approach a constant value independent of energy. The low energy behavior of the cross sections can be recovered by replacing the structure functions by form factor dependent terms. The factor $A$ is the same for neutrino and antineutrino probes scattering off an isoscalar target (up to a small correct for quark mixing as described below). The magnitude of the coefficient $B$ is larger for antineutrino, since $F_{2}$ and $x F_{3}$ terms have the same sign for antineutrino in Eq. 3. We use a correction computed from the generator level cross section model to account for a finite $\nu_{0}>0$ value

$$
S^{\nu(\bar{\nu})}\left(\nu_{0}, E\right)=1+\int_{0}^{\nu_{0}} \frac{B^{\nu, \bar{\nu}}}{A} \frac{\nu}{E}-\int_{0}^{\nu_{0}} \frac{C^{\nu, \bar{\nu}}}{A} \frac{\nu^{2}}{2 E^{2}},
$$

As described by Eq. 6.2, as $\nu \rightarrow 0$, in absence of quark mixing, $\sigma^{\nu}=\sigma^{\bar{\nu}}$. However, if quark mixing is accounted for, the structure function $F_{2}$ is not exactly the same for neutrinos and antineutrinos as shown by Eq. 1.34 and Eq. 1.35. The difference of neutrino and antineutrino structure functions is written as

$$
\Delta F_{2}=F_{2}^{\nu}-F_{2}^{\bar{\nu}}=V_{c d}^{2} \frac{u_{v}+d_{v}}{2}-\left(V_{u s}^{2} \frac{u_{s}+d_{s}}{2}+s_{s} V_{v s}^{2}\right)
$$

An additional correction is applied when normalizing the antineutrino cross section to account for $\Delta F_{2}$. This $\nu$-dependent correction is computed from model using

$$
G_{c o r r}\left(\nu_{0}\right)=\frac{\sigma^{\bar{\nu}}\left(\nu<\nu_{0}, E \rightarrow \infty\right)}{\sigma^{\nu}\left(\nu<\nu_{0}, E \rightarrow \infty\right)} .
$$




\subsection{CROSS SECTION AND FLUX EXTRACTION}

The charged-current total cross sections are measured from the inclusive charged-current scattering rate, $\Gamma_{\mathrm{CC}}^{\nu(\bar{\nu})}(E)$, and the incident neutrino flux, $\Phi^{\nu(\bar{\nu})}(E)$. The selected CC event sample, $N_{\mathrm{CC}}^{\nu(\bar{\nu})}(E)$, is corrected for acceptance and backgrounds to determine $\Gamma_{\mathrm{CC}}^{\nu(\bar{\nu})}(E)$ :

$$
\Gamma_{\mathrm{CC}}^{\nu(\bar{\nu})}(E)=\left(N_{\mathrm{CC}}^{\nu(\bar{\nu})}(E)-B_{\mathrm{CC}}^{\nu(\bar{\nu})}(E)\right) \times A_{\mathrm{CC}}^{\nu(\bar{\nu})}(E)
$$

The raw flux sample is also corrected for backgrounds and acceptance

$$
\Phi_{u c}^{\nu(\bar{\nu})}(E)=\left(F^{\nu(\bar{\nu})}(E)-B_{\Phi}^{\nu(\bar{\nu})}(E)\right) \times A_{\Phi}^{\nu(\bar{\nu})}(E) .
$$

Then the model-based $S^{\nu(\bar{\nu})}\left(\nu_{0}, E\right)$ and normalization $H_{i s o}^{\nu(\bar{\nu})}\left(\nu_{o}\right)$ corrections are applied to obtain $\Phi^{\nu(\bar{\nu})}(E)$.

$$
\Phi^{\nu(\bar{\nu})}(E)=H^{\nu(\bar{\nu})}\left(\nu_{o}\right) \Phi_{u c}^{\nu(\bar{\nu})}(E) / S^{\nu(\bar{\nu})}\left(\nu_{0}, E\right) .
$$

$H^{\nu}\left(\nu_{0}\right)$ and $H^{\bar{\nu}}\left(\nu_{0}\right)$ are related by $G_{c o r r}\left(\nu_{0}\right)$ defined in Eq. 6.8 and $\alpha\left(\nu_{0}\right)$, which is defined in Sec. 6.5.1. The measured cross sections are obtained from

$$
\frac{\sigma_{C C}^{\nu(\bar{\nu})}(E)}{E}=\frac{1}{E} \frac{\Gamma_{\mathrm{CC}}^{\nu(\bar{\nu})}(E)}{\Phi^{\nu(\bar{\nu})}(E)}=\frac{1}{E}\left(\frac{\left(N_{\mathrm{CC}}^{\nu(\bar{\nu})}(E)-B_{\mathrm{CC}}^{\nu(\bar{\nu})}(E)\right) \times A_{\mathrm{CC}}^{\nu(\bar{\nu})}(E)}{H^{\nu(\bar{\nu})}\left(\nu_{o}\right)\left(F^{\nu(\bar{\nu})}(E)-B_{\Phi}^{\nu(\bar{\nu})}(E)\right) \times A_{\Phi}^{\nu(\bar{\nu})}(E) / S^{\nu(\bar{\nu})}\left(\nu_{0}, E\right)}\right) .
$$

The components in Eq. 6.12 are

- $N_{\mathrm{CC}}^{\nu(\bar{\nu})}(E)$ is the selected CC event sample and $F^{\nu(\bar{\nu})}(E)$ is the flux sample, which are shown in Fig. 41.

- $B_{\mathrm{CC}}^{\nu(\bar{\nu})}(E)$ and $B_{\Phi}^{\nu(\bar{\nu})}(E)$ are the backgrounds in the inclusive-CC and flux samples respectively, which are shown in Fig. 48 and Fig. 49. For each sample, the background includes wrong-sign contamination and neutral-current $(\mathrm{NC})$ contamination. $\mathrm{NC}$ contamination is small $(<1 \%)$ for both neutrino and antineutrino samples. However, wrong-sign contamination for the RHC antineutrino inclusive sample is non-negligible as shown on the right of Fig. 49 (about 3.5\% at $10 \mathrm{GeV}$ and larger above that), a systematic uncertainty is included for this in the antineutrino cross section and the ratio, $r$. 
- $A_{\mathrm{CC}}^{\nu(\bar{\nu})}(E)$ corrects for acceptance and smearing effects in the raw samples and is obtained from the Monte Carlo simulation

$$
A_{\mathrm{CC}}^{\nu(\bar{\nu})}(E)=\frac{N_{\text {true }}^{\nu(\bar{\nu})}\left(E_{i}\right)}{N_{\text {reco }}^{\nu(\bar{\nu})}\left(E_{i}\right)},
$$

where $N_{\text {true }}^{\nu(\bar{\nu})}\left(E_{i}\right)$ is the number of true events in an energy bin $E_{i}$ (in true neutrino energy) and $N_{\text {reco }}^{\nu(\bar{\nu})}\left(E_{i}\right)$ is the number of events reconstructed in that bin (in reconstructed neutrino energy) passing all the selection cuts described for the $N_{\mathrm{CC}}^{\nu(\bar{\nu})}(E)$ event sample. $A_{\mathrm{CC}}^{\nu(\bar{\nu})}(E)$ can be written as the product of two contributions.

$$
A_{\mathrm{CC}}^{\nu(\bar{\nu})}(E)=A^{\nu(\bar{\nu}), \mathrm{KIN}} \times A_{C C}^{\nu(\bar{\nu}), \mathrm{DET}} .
$$

The $A_{C C}^{\mathrm{DET}}$ term accounts primarily for detector resolution and reconstruction smearing effects rather than geometric acceptance. It uses the MINER $\nu \mathrm{A}$ GEANT4-based detector simulation with GENIE 2.8.4 event generator to provide an underlying event distribution that populates regions where the detector has good geometric acceptance. This correction also has a model dependence arising from final state interactions (FSI) that result in bin migration. We used GENIE 2.8.4 model to compute $A_{C C}^{\mathrm{DET}}$ as shown in Fig. 51. The kinematic correction $A^{\nu(\bar{\nu}), \mathrm{KIN}}$ will be described in detail in Sec. 6.3.2.

- $A_{\Phi}^{\nu(\bar{\nu})}(E)$ corrects for acceptance and smearing in the flux sample. Therefore, no separate kinematic correction is needed in this case. The maximum $\nu$ requirement selects muons which are scattered in the forward direction and are all above the minimum energy threshold even for our lowest binned energy 2-3 GeV. $A_{\Phi}^{\nu(\bar{\nu})}(E)$ is obtained from

$$
A_{\Phi}^{\nu(\bar{\nu})}(E)=\frac{F_{\text {true }}^{\nu(\bar{\nu})}\left(E_{i}\right)}{F_{\text {reco }}^{\nu(\bar{\nu})}\left(E_{i}\right)}
$$

where $F_{\text {true }}^{\nu(\bar{\nu})}\left(E_{i}\right)$ is the number of true events with true hadron energy cut in an energy bin $E_{i}$ (in true neutrino energy) and $F_{\text {reco }}^{\nu(\bar{\nu})}\left(E_{i}\right)$ is the number of events reconstructed in that bin (in reconstructed neutrino energy) passing all the selection cuts described for the $F^{\nu(\bar{\nu})}(E)$ sample. $A_{\Phi}^{\nu(\bar{\nu})}(E)$ is shown in Fig. 51. 

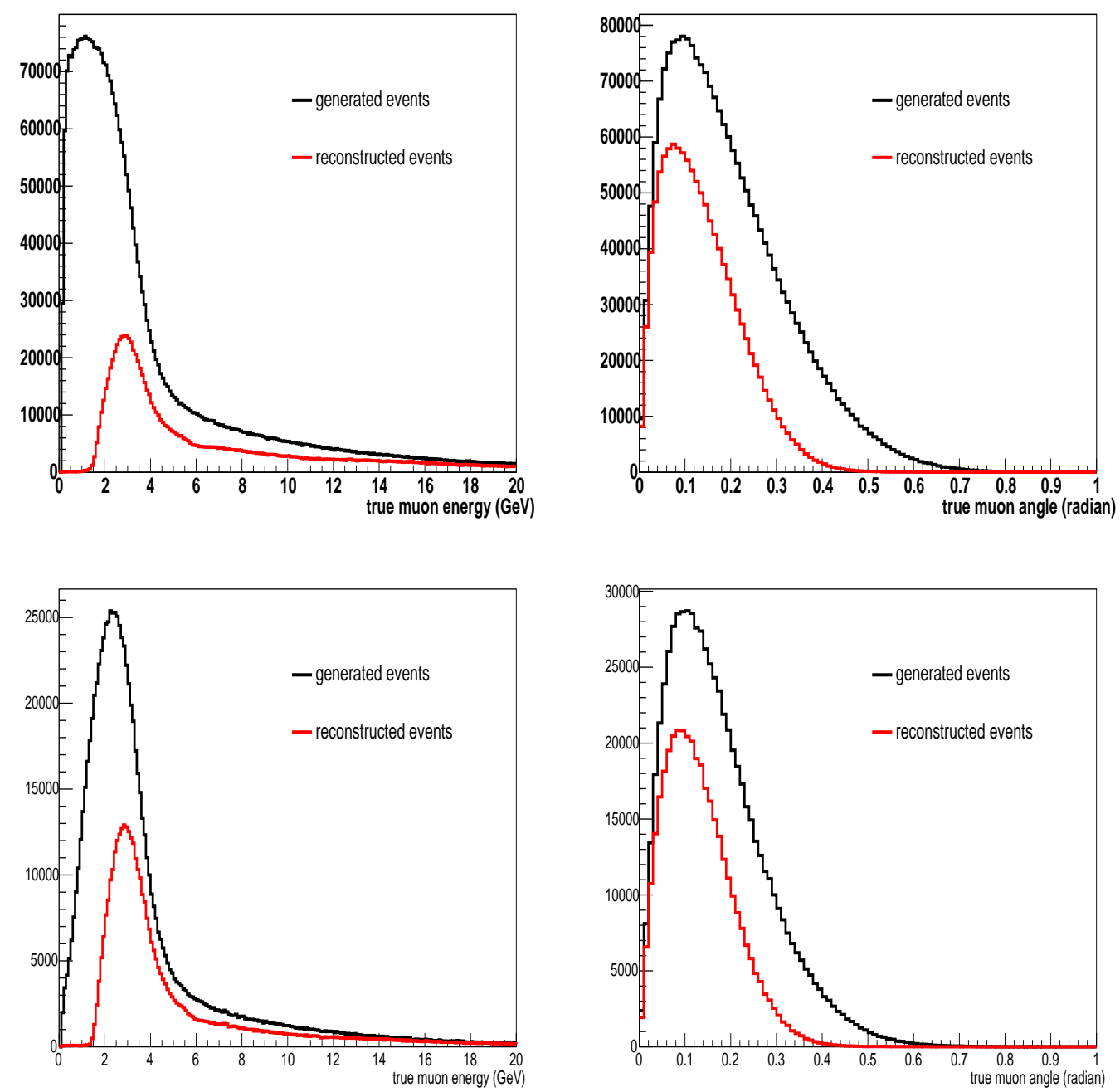

Figure 50: (Left) Distribution of true muon energy for generated and reconstructed events. (Right) Distribution of true muon angle w.r.t beam for generated and reconstructed events, both with an minimal true muon energy cut of $1.8 \mathrm{GeV}$ applied. Top figures show neutrino samples for all neutrino energies. Bottom figures show antineutrino samples for all neutrino energies.

Before applying any generator level corrections we define

$$
R_{C}^{\nu(\bar{\nu})}=\frac{\left(N_{\mathrm{CC}}^{\nu(\bar{\nu})}(E)-B_{\mathrm{CC}}^{\nu(\bar{\nu})}(E)\right) \times A_{C C}^{\nu(\bar{\nu}), \text { DET }}(E)}{\left(F^{\nu(\bar{\nu})}(E)-B_{\Phi}^{\nu(\bar{\nu})}(E)\right) \times A_{\Phi}^{\nu(\bar{\nu})}(E)},
$$

which includes all the terms discussed in this section. 

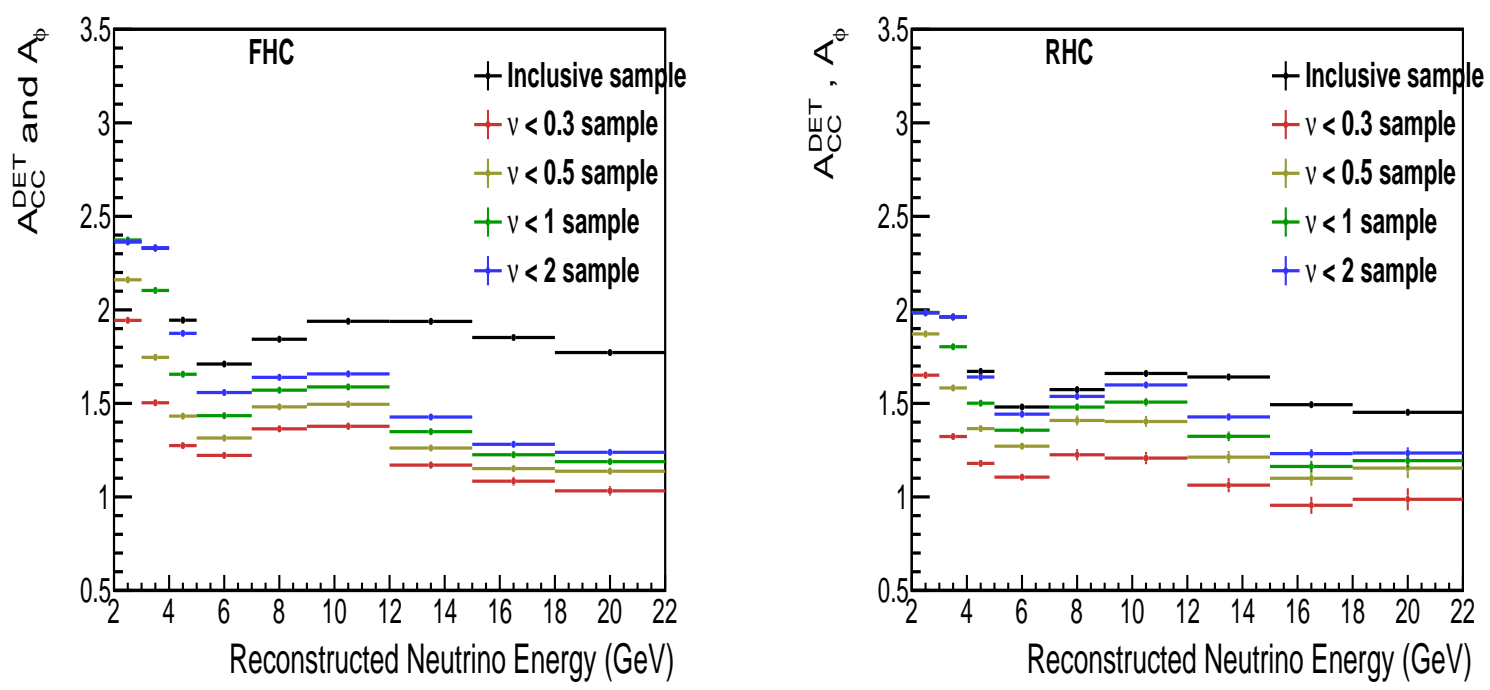

Figure 51: $A_{C C}^{\mathrm{DET}}$ for cross section and $A_{\Phi}\left(E_{i}\right)$ for flux samples of FHC neutrinos (left) and RHC antineutrinos (right).

\subsection{GENERATOR LEVEL MODEL-DEPENDENT CORRECTIONS}

We rewrite Eq. 6.12 by separating out all model dependent components, and the neutrino (antineutrino) cross section becomes

$$
\frac{\sigma_{C C}^{\nu(\bar{\nu})}(E)}{E}=\frac{1}{E} \times R_{C}^{\nu(\bar{\nu})} \times \frac{A_{C C}^{\nu(\bar{\nu}), \mathrm{KIN}}(E) \times S^{\nu(\bar{\nu})}\left(\nu_{0}, E\right)}{H^{\nu(\bar{\nu})}\left(\nu_{o}\right)}
$$

Two independent neutrino Monte Carlo generator models are used for computing the model dependent corrections, low- $\nu$ correction $S^{\nu}$, kinematic correction $A_{C C}^{K I N}$, and isoscalar correction $I^{i s o}$. Our primary result uses the GENIE-Hybrid model which is described in Sec. 3.1.

\subsubsection{Low $-\nu$ correction}

The low- $\nu$ correction $S^{\nu(\bar{\nu})}\left(\nu_{0}, E\right)$, described in Sec. 6.1, is computed directly from a model using

$$
S^{\nu(\bar{\nu})}\left(\nu_{0}, E\right)=\frac{\sigma^{\nu(\bar{\nu})}\left(\nu<\nu_{o}, E\right)}{\sigma^{\nu(\bar{\nu})}\left(\nu<\nu_{o}, E \rightarrow \infty\right)} .
$$


The term $\sigma^{\nu(\bar{\nu})}\left(\nu<\nu_{0}, E\right)$ is the value of the integrated cross section (on carbon 12) below our chosen $\nu_{0}$ cut at energy $E$, and $\sigma^{\nu(\bar{\nu})}\left(\nu<\nu_{0}, E \rightarrow \infty\right)$ is its value in the high energy limit. The size of this correction is modest for neutrinos but sizable for antineutrinos.

\subsubsection{Kinematic Correction}

As shown in Eq. 6.14, we define two terms (for both neutrino and antineutrino samples),

$$
A_{C C}^{\nu(\bar{\nu}), \mathrm{DET}}=\frac{N_{\text {true }}^{\nu(\bar{\nu})}\left(E_{i}, E_{\mu}>E_{\mu \min }, \theta_{\mu}<\theta_{\mu \max }\right)}{N_{\text {reco }}^{\nu(\bar{\nu})}\left(E_{i}\right)}
$$

and

$$
A^{\nu(\bar{\nu}), \mathrm{KIN}}=\frac{N_{\text {true }}^{\nu(\bar{\nu})}\left(E_{i}\right)}{N_{\text {true }}^{\nu(\bar{\nu})}\left(E_{i}, E_{\mu}>E_{\mu \min }, \theta_{\mu}<\theta_{\mu \max }\right)} .
$$

$N_{\text {true }}^{\nu(\bar{\nu})}\left(E_{i}, E_{\mu}>E_{\mu \min }, \theta_{\mu}<\theta_{\mu \max }\right)$ is a subset of $N_{\text {true }}^{\nu(\bar{\nu})}\left(E_{i}\right)$, which passes additional minimal true muon energy cut and maximum true muon angle cut as described below. $A^{\nu(\bar{\nu}), \mathrm{KIN}}$ is computed by calculating the ratio of the number of all generated $\mathrm{CC}$ neutrino events to number of generated $\mathrm{CC}$ neutrino events with a minimum muon energy cut $E_{\mu m i n}=1.8 \mathrm{GeV}$ and a maximum muon angle cut $\theta_{\mu \max }=0.35$ rads. The minimum energy requirement excludes events which are not energetic enough to penetrate the MINOS Near detector and produce a measureable track while the maximum angle requirement removes those outside of the MINOS angular acceptance. Fig. 50 shows the distribution of true muon energy of generated and selected events (left), and the true muon angle and component with reconstructed muon angle after applying the minimal muon energy cut $E_{\mu}>1.8 \mathrm{GeV}$ (right) for FHC neutrinos and RHC antineutrinos. Tab. 17 shows the fraction of events removed by kinematic cuts for both neutrino and antineutrino samples. The contribution from events with muon energy below the threshold needed to obtain total cross section is large at low energy and must be computed from a model.

\subsubsection{Isoscalar correction}

The MINER $\nu \mathrm{A}$ scintillator target requires a model dependent non-isoscalarity correction to account for the proton excess from the hydrogen bound in scintillator. Tab. 18 shows the composition 
by mass of a MINER $\nu \mathrm{A}$ scintillator plane [8]. Since this correction is nearly flat with energy, the primary effect is on the normalization (discussed in Sec. 7.1).

We define the fraction of proton and fraction of neutron in scintillator plane as

$$
f_{\text {proton }}=\frac{Z}{N+Z}
$$

and

$$
f_{\text {neutron }}=\frac{N}{N+Z}
$$

respectively. We compute the fractions from Tab. 18 and obtain the fractions $f_{\text {proton }}=53.97 \%$, and $f_{\text {neutron }}=46.03 \%$. The isoscalar correction is defined as

$$
I_{i s o}^{\nu(\bar{\nu})}\left(\nu_{0}, E\right)=\frac{J_{i s o}^{\nu(\bar{\nu})}(E)}{J_{i s o}^{\nu(\bar{\nu})}\left(\nu_{0}, E\right)}
$$

where $J_{i s o}^{\nu(\bar{\nu})}(E)$ is the isoscalar correction for the total cross section and $J_{i s o}^{\nu(\bar{\nu})}\left(\nu_{0}, E\right)$ is the isoscalar correction for the flux sample.

The isoscalar correction for the total cross section is defined as

$$
J_{i s o}^{\nu(\bar{\nu})}(E)=\frac{\sigma_{i s o}^{\nu(\bar{\nu})}(E)}{\sigma_{\text {real }}^{\nu(\bar{\nu})}(E)}
$$

in which $\sigma_{i s o}^{\nu(\bar{\nu})}(E)$ is the isoscalar corrected cross section which has equal contributions from proton and neutron,

$$
\sigma_{\text {iso }}^{\nu(\bar{\nu})}(E)=\frac{\sigma_{\text {proton }}^{\nu(\bar{\nu})}(E)+\sigma_{\text {neutron }}^{\nu(\bar{\nu})}(E)}{2}
$$

and $\sigma_{\text {real }}^{\nu(\bar{\nu})}$ is the cross section on real scintillator,

$$
\begin{gathered}
\sigma_{\text {real }}^{\nu(\bar{\nu})}(E)=\frac{\sigma_{\text {proton }}^{\nu(\bar{\nu})}(E) Z+\sigma_{\text {neutron }}^{\nu(\bar{\nu})}(E) N}{N+Z} \\
\sigma_{\text {real }}^{\nu(\bar{\nu})}=\sigma_{\text {proton }}^{\nu(\bar{\nu})}(E) f_{\text {proton }}+\sigma_{\text {neutron }}^{\nu(\bar{\nu})}(E) f_{\text {neutron }}
\end{gathered}
$$

Therefore, the isoscalar correction for the cross section is calculated as

$$
J_{\text {iso }}^{\nu(\bar{\nu})}(E)=\frac{\frac{1}{2}\left(\sigma_{\text {proton }}^{\nu(\bar{\nu})}(E)+\sigma_{\text {neutron }}^{\nu(\bar{\nu})}(E)\right)}{f_{\text {proton }} \sigma_{\text {proton }}^{\nu(\bar{\nu})}(E)+f_{\text {neutron }} \sigma_{\text {neutron }}^{\nu(\bar{\nu})}(E)}
$$


where $\sigma_{\text {proton }}^{\nu(\bar{\nu})}(E)$ is the neutrino (antineutrino) cross section on a proton in carbon 12, and $\sigma_{\text {neutron }}^{\nu(\bar{\nu})}(E)$ is the neutrino (antineutrino) cross section on a neutron in carbon 12.

The $\nu_{0}$-dependent isoscalar corrections for the flux samples are calculated similarly as

$$
J_{\text {iso }}^{\nu(\bar{\nu})}\left(\nu_{0}, E\right)=\frac{\frac{1}{2}\left(\sigma_{\text {proton }}^{\nu(\bar{\nu})}\left(\nu_{0}, E\right)+\sigma_{\text {neutron }}^{\nu(\bar{\nu})}\left(\nu_{0}, E\right)\right)}{f_{\text {proton }} \sigma_{\text {proton }}^{\nu(\bar{\nu})}\left(\nu_{0}, E\right)+f_{\text {neutron }} \sigma_{\text {neutron }}^{\nu(\bar{\nu})}\left(\nu_{0}, E\right)}
$$

where $\sigma_{\text {proton }}^{\nu(\bar{\nu})}\left(\nu_{0}, E\right)$ is the neutrino (antineutrino) cross section on a proton in carbon 12 with $\nu<\nu_{0}$, and $\sigma_{\text {neutron }}^{\nu(\bar{\nu})}\left(\nu_{0}, E\right)$ is the neutrino (antineutrino) cross section on a neutron in carbon 12 with $\nu<\nu_{0}$.

GENIE Hybrid $G_{\text {corr }}$ corrections are listed in Tab. 23.

\subsection{NUWRO-BASED MODEL-DEPENDENT CORRECTIONS}

We also extract cross sections using NuWro-based Corrections. Since GENIE 2.8.4 with FSI (turned on by default) is used to simulate the fully reconstructed MINER $\nu \mathrm{A}$ MC samples and to correct for detector effects we deliberately turn the FSI processes off in NuWro to avoid double counting. In this section, we present the model-dependent correction calculated from NuWro, and the comparison of GENIE-Hybrid-based and NuWro-based corrections.

A comparison of GENIE-based and NuWro-based low $-\nu$ corrections is shown in Fig. 53. The NuWro-based low- $\nu$ correction is calculated by counting the number of events which passes a $\nu<\nu_{0}$ cut, $N\left(E, \nu<\nu_{0}\right)$. Then by computing

$$
\sigma^{\nu(\bar{\nu})}\left(\nu<\nu_{0}, E_{i}\right)=\frac{N\left(E_{i}, \nu<\nu_{0}\right)}{N\left(E_{i}\right)} \times \sigma\left(E_{i}\right)
$$

$N\left(E_{i}\right)$ is the number of events generated at each energy.

Fig. 52 shows a comparison of GENIE-Hybrid and NuWro-based kinematic correction $A_{C C}^{K I N}$ for neutrinos (left) and antineutrinos (right). The corrections differ by less than $1 \%$ except for in the first neutrino energy bin. The differences are due to QEL and RES model kinematics in the two models. 
In order to understand the difference in $A_{C C}^{K I N}$ correction between GENIE-Hybrid and NuWro, we break down the hadron energy distributions based on interaction types for the numerator and denominator of $A_{C C}^{K I N}$ in the energy region $2<E_{\nu}<3 \mathrm{GeV}$, which are shown in Fig. 54 for neutrino and Fig. 55 for antineutrino. Due to different $M_{A}$ values and kinematic modeling, NuWro has $8 \%$ more QEL events generated in the first neutrino energy bin, $2<E_{\nu}<3 \mathrm{GeV}$. The MEC model used is different between GENIE (Nieves) and NuWro (TEM), which also contributes to the $A_{C C}^{K I N}$ difference. In the first neutrino energy bin $2<E_{\nu}<3 \mathrm{GeV}$, QEL and MEC events dominate. NuWro has better acceptance than GENIE for these events, which results in a smaller $A_{C C}^{K I N}$. As neutrino energy increases, above $3 \mathrm{GeV}$, DIS and RES pieces become dominant and GENIE has better acceptance.

Fig. 56 shows the isoscalar correction calculated from GENIE and NuWro, which includes isoscalar corrections for both inclusive and flux samples. It is a step function because the isoscalar correction for flux sample is strongly $\nu_{0}$ dependent, and $\nu_{0}$ differs depending on the neutrino energy range. These corrections are nearly identical in the two models.

We do not report $G_{\text {corr }}$ in NuWro, since quark mixing is not included in NuWro. GENIEHybrid-Based $G_{c o r r}$ corrections are applied to NuWro-based final results, as shown in Sec. 8.4.1.

The model dependent corrections needed to extract neutrino cross sections in the low- $\nu$ method described above are tabulated for the GENIE Hybrid model in Tab. 19 and NuWro in Tab. 20. Those used to extract antineutrino cross section are in Tab. 21 and Tab. 22.

\subsection{FLUX NORMALIZATION}

The normalization procedure couples the flux measurement to the cross section sample and relies on external information to obtain a value for the neutrino normalization factor $H^{\nu}\left(\nu_{0}\right)$. Since neutrino and antineutrino scattering rates are related (as described in Sec 6.1), as described further below, the antineutrino normalization $\left(H^{\bar{\nu}}\left(\nu_{0}=2\right)\right)$ is obtained using the same precise external neutrino scattering measurements. 
The isoscalar corrected total neutrino cross section is written,

$$
\begin{gathered}
\frac{\sigma_{C C}^{\nu}(E)_{i s o}}{E}=\frac{1}{E}\left(\frac{\left(N_{\mathrm{CC}}^{\nu}(E)-B_{\mathrm{CC}}^{\nu}(E)\right) \times A_{\mathrm{CC}}^{\nu}(E) \times S^{\nu}\left(\nu_{0}, E\right)}{\left(F^{\nu}(E)-B_{\Phi}^{\nu}(E)\right) \times A_{\Phi}^{\nu}(E)}\right) \frac{I_{i s o}^{\nu}\left(\nu_{0}, E\right)}{H_{i s o}^{\nu}\left(\nu_{o}\right)} . \\
\frac{\sigma_{C C}^{\nu(\bar{\nu})}(E)_{i s o}}{E}=\frac{1}{E} \times R_{C}^{\nu(\bar{\nu})} \times \frac{A_{C C}^{\nu(\bar{\nu}), \mathrm{KIN}}\left(E_{i}\right) \times S^{\nu(\bar{\nu})}\left(\nu_{0}, E\right) \times I_{i s o}^{\nu(\bar{\nu})}\left(\nu_{0}, E\right)}{H_{i s o}^{\nu(\bar{\nu})}\left(\nu_{o}\right)} .
\end{gathered}
$$

The procedure for computing $I_{i s o}^{\nu(\bar{\nu})}\left(\nu_{0}, E\right)$ from a given neutrino generator model is given in Sec. 6.3.3. After the isoscalar correction is applied, the unnormalized neutrino cross section obtained using flux with $\nu<\nu_{0}$ at energy $\mathrm{E}$ is written as

$$
\sigma_{C C}^{\nu(\bar{\nu})}\left(\nu_{o}, E_{i}\right)_{\text {unnorm }}=\frac{1}{E} \times R_{C}^{\nu(\bar{\nu})} \times A_{C C}^{\nu(\bar{\nu}), \mathrm{KIN}}\left(E_{i}\right) \times S^{\nu(\bar{\nu})}\left(\nu_{0}, E\right) \times I_{i s o}^{\nu(\bar{\nu})}\left(\nu_{0}, E\right)
$$

The normalization factor for neutrino cross section, $H_{i s o}^{\nu}\left(\nu_{o}\right)$, is computed using the unnormalized data cross section in the energy bin $12-22 \mathrm{GeV}$ (at $17 \mathrm{GeV}), \sigma_{\text {unnorm }}^{\nu}(E=17 \mathrm{GeV})$,

$$
\frac{1}{H_{\text {iso }}^{\nu}\left(\nu_{0}\right)}=\frac{\sigma_{E X T}(E=17 G e V)}{\sigma_{\text {unnorm }}\left(\nu_{0}, E=17 G e V\right)}
$$

where $\sigma_{E X T}$ is obtained from external world data (discussed in Sec. 6.5.2). The uncertainty in normalization is also obtained from the external data and must be propagated through the analysis. The normalization value must be obtained for each $\nu_{0}$ cut. To do this we measure a value of $\sigma_{\text {unnorm }}\left(\nu_{0}, E=17 \mathrm{GeV}\right)$ for each $\nu_{0}$ value. Tab. 15 gives the values of $\Phi_{u c}^{\nu}(E)$ for each $\nu_{0}$ value needed as input to obtain $H_{i s o}^{\nu}\left(\nu_{o}\right)$.

The normalization value $H_{i s o}^{\nu}\left(\nu_{o}\right)$ is obtained after the model dependent corrections $S^{\nu}\left(\nu_{0}, E\right)$, $I_{i s o}^{\nu}\left(\nu_{0}, E\right)$, and $A_{C C}^{K I N}(E)$ are applied to the data in Tab. 13 and 15. 


\subsubsection{Antineutrino Cross Section Normalization}

The factor, $H_{i s o}^{\bar{\nu}}\left(\nu_{o}\right)$, is applied to the isoscalar corrected antineutrino cross section

$$
\frac{\sigma_{C C}^{\bar{\nu}}(E)}{E}=\frac{1}{E} \times R_{C}^{\bar{\nu}} \frac{A_{C C}^{\bar{\nu}, K I N}(E) \times S^{\bar{\nu}}\left(\nu_{0}, E\right) \times I_{i s o}^{\bar{\nu}}\left(\nu_{0}, E\right)}{H_{\text {iso }}^{\bar{\nu}}\left(\nu_{o}\right)} .
$$

$H_{i s o}^{\bar{\nu}}\left(\nu_{o}\right)$ is related to $H_{i s o}^{\nu}\left(\nu_{o}\right)$ by

$$
H_{\text {iso }}^{\nu}\left(\nu_{o}\right)=H_{i s o}^{\bar{\nu}}\left(\nu_{o}\right) \times G_{\text {corr }}\left(\nu_{0}\right) \times \alpha\left(\nu_{0}\right),
$$

where $G_{c o r r}\left(\nu_{0}\right)$ is the quark mixing correction defined in Eq. 6.8 and $\alpha\left(\nu_{0}\right)$ is an additional correction obtained from low- $\nu$ antineutrino data.

The correction $\alpha\left(\nu_{0}\right)$ is needed to account for unmodeled $\nu$ dependent cross section contributions that are observed in our data. If uncorrected, they result in differences in flux event sample rates that are inconsistent in the normalization bin $(12-22 \mathrm{GeV})$. This is demonstrated in Fig. 57 which shows the extracted antineutrino cross section (normalized as in Eq.6.36 but with $\alpha\left(\nu_{0}\right)=1$ ) obtained for each value $\nu_{o}$ applied across all energy bins. For each $\nu_{o}$ value a shift in the level of the cross section in the normalization region $(12-22 \mathrm{GeV})$ is observed. To remedy this, we constrain the measured antineutrino cross section in the normalization bin to agree across all $\nu_{o}$ values by define $\alpha\left(\nu_{0}\right)$ for each $\nu_{0}$ using

$$
G_{\text {corr }}\left(\nu_{0}\right) \alpha\left(\nu_{0}\right) \frac{\sigma_{C C}^{\bar{\nu}}\left(\nu_{0}, E=17 G e V\right)_{\text {unnorm }}}{H_{\text {iso }}^{\nu}\left(\nu_{0}\right)}=G_{\text {corr }}\left(\nu_{0}=2\right) \frac{\sigma_{C C}^{\bar{\nu}}\left(\nu_{0}=2, E=17 G e V\right)_{\text {unnorm }}}{H_{\text {iso }}^{\nu}\left(\nu_{0}=2\right)} .
$$

To preserve the relationship between neutrino and antineutrino cross sections defined in Sec. 6.1 we apply define $\alpha\left(\nu_{0}\right)=1$ for the $\nu_{0}=2$ sample in Eq. 6.36. The antineutrino cross section is then

$$
\sigma_{C C}^{\bar{\nu}}\left(E_{i}\right)=\frac{\sigma_{C C}^{\bar{\nu}}\left(E_{i}\right)_{\text {unnorm }}}{H_{\text {iso }}^{\nu}\left(\nu_{o}\right)} \times G_{c o r r}\left(\nu_{0}\right) \times \alpha\left(\nu_{0}\right) .
$$

The overall normalization factor $H_{i s o}^{\nu}\left(\nu_{o}\right)$ cancels in $r$ which becomes

$$
r=\frac{\sigma_{C C}^{\bar{\nu}}\left(E_{i}\right)}{\sigma_{C C}^{\nu}(E)}=\frac{\sigma_{C C}^{\bar{\nu}}\left(E_{i}\right)_{\text {unnorm }}}{\sigma_{C C}^{\nu}(E)_{\text {unnorm }}} \times G_{\text {corr }}\left(\nu_{0}\right) \times \alpha\left(\nu_{0}\right) .
$$

Tab 24 shows the size of $\alpha\left(\nu_{0}\right)$ obtained from our data sample and its uncertainty for different $\nu$ cuts. The statistical error of unnormalized antineutrino cross section at $17 \mathrm{GeV}, \sigma_{C C}^{\bar{\nu}}\left(\nu<\nu_{0}, E=\right.$ $17 \mathrm{GeV})_{\text {unnorm }}$, is assigned as the uncertainty of $\alpha\left(\nu_{0}\right)$. 


\subsubsection{External Cross Section Measurements between 12 and $22 \mathrm{GeV}$ (normalization bin).}

Fig. 58 shows the world data of CC inclusive neutrino cross section in our normalization bin of $12-22 \mathrm{GeV}$. Table 25 shows the most precise measurements from MINOS and NOMAD. All cross sections are isoscalar corrected. We normalize our neutrino cross section in the $12-22 \mathrm{GeV}$ energy bin to the NOMAD measurement which has an average value $0.699 \times 10^{-38} \mathrm{~cm}^{2} / \mathrm{GeV}$ with an uncertainty of $3.58 \%$. We use NOMAD for several reasons. It is a measurement of the neutrino cross section on an isoscalar carbon target, and it is the most precise measurement in our normalization energy range. MINOS also measures neutrino cross section in $12-22 \mathrm{GeV}$ energy range, but uses a heavier (iron) target. Also, because the MINOS near detector is used to measure the muon momentum in $\operatorname{MINER} \nu \mathrm{A}$, the results are partially correlated. To avoid accounting for this, we do not include the MINOS measurement in our "external" normalization. 


\begin{tabular}{|c|c|c|c|c|c|c|c|c|}
\hline \hline $\mathrm{E}(\mathrm{GeV})$ & $N_{C C}^{\nu}$ & $\begin{array}{c}\text { stat. err. } \\
(\%)\end{array}$ & $B_{C C}^{\nu}$ & $A_{C C}^{\mathrm{DET}}$ & $F^{\nu}$ & $\begin{array}{c}\text { stat. err } \\
(\%)\end{array}$ & $B_{\Phi}^{\nu}$ & $A_{\phi}^{\nu}$ \\
\hline 2.5 & 20660.0 & 0.70 & 52.92 & 2.38 & 11493.0 & 0.93 & 29.17 & 1.94 \\
\hline 3.5 & 44360.0 & 0.47 & 61.20 & 2.30 & 25530.0 & 0.63 & 19.23 & 1.76 \\
4.5 & 29586.0 & 0.58 & 64.78 & 1.92 & 11765.0 & 0.92 & 12.95 & 1.45 \\
6.0 & 32026.0 & 0.56 & 169.65 & 1.70 & 8046.0 & 1.11 & 28.80 & 1.34 \\
\hline 8.0 & 23750.0 & 0.65 & 171.05 & 1.86 & 6980.0 & 1.20 & 31.97 & 1.59 \\
10.5 & 29161.0 & 0.59 & 207.48 & 1.95 & 6165.0 & 1.27 & 31.26 & 1.60 \\
\hline 13.5 & 24093.0 & 0.64 & 158.49 & 1.94 & 7438.0 & 1.16 & 39.49 & 1.42 \\
16.5 & 19011.0 & 0.73 & 104.46 & 1.85 & 5041.0 & 1.41 & 17.23 & 1.28 \\
20.0 & 18475.0 & 0.74 & 98.26 & 1.78 & 3826.0 & 1.62 & 13.55 & 1.25 \\
\hline \hline
\end{tabular}

Table 13: Measured neutrino cross section and flux sample yields corrected for wrong-sign and neutral current background contaminations. The $A_{C C}^{\mathrm{DET}}$ correction term accounts for reconstruction resolution and smearing effects but not for events outside of the acceptance . (Additional model dependent corrections for kinematic regions where the spectrometer has no acceptance and for energy dependence in the low- $\nu$ sample must be applied to compute the total cross sections as described in the text). 


\begin{tabular}{|c|c|c|c|c|c|c|c|c|}
\hline \hline $\mathrm{E}(\mathrm{GeV})$ & $N_{C C}^{\nu}$ & $\begin{array}{c}\text { stat. err. } \\
(\%)\end{array}$ & $B_{C C}^{\nu}$ & $A_{C C}^{\mathrm{DET}}$ & $F^{\nu}$ & $\begin{array}{c}\text { stat. err } \\
(\%)\end{array}$ & $B_{\Phi}^{\nu}$ & $A_{\phi}^{\nu}$ \\
\hline 2.50 & 5359.00 & 1.37 & 17.79 & 1.99 & 3673.00 & 1.65 & 6.37 & 1.60 \\
\hline 3.50 & 10133.00 & 0.99 & 24.88 & 1.94 & 6560.00 & 1.23 & 4.33 & 1.56 \\
4.50 & 5955.00 & 1.30 & 24.42 & 1.65 & 2871.00 & 1.87 & 1.68 & 1.36 \\
6.00 & 5284.00 & 1.38 & 73.73 & 1.47 & 1764.00 & 2.38 & 4.13 & 1.27 \\
\hline 8.00 & 3261.00 & 1.75 & 101.68 & 1.58 & 1224.00 & 2.86 & 5.61 & 1.50 \\
10.50 & 3400.00 & 1.71 & 141.36 & 1.66 & 1007.00 & 3.15 & 8.75 & 1.53 \\
\hline 13.50 & 2496.00 & 2.00 & 115.01 & 1.63 & 1033.00 & 3.11 & 8.85 & 1.42 \\
16.50 & 1690.00 & 2.43 & 76.73 & 1.48 & 595.00 & 4.10 & 5.56 & 1.23 \\
20.00 & 1418.00 & 2.66 & 72.34 & 1.44 & 427.00 & 4.84 & 5.00 & 1.23 \\
\hline \hline
\end{tabular}

Table 14: Antineutrino measured cross section and flux sample rates corrected for wrong sign and neutral current background contaminations. 


\begin{tabular}{|c|c|c|c|c|c|}
\hline \hline$\nu$ cut $(\mathrm{GeV})$ & $\mathrm{E}(\mathrm{GeV})$ & $F^{\nu(\bar{\nu})}(E)$ & $\operatorname{stat}(\%)$ & $B_{\Phi}^{\nu(\bar{\nu})}(E)$ & $A_{\phi}$ \\
\hline \multirow{3}{*}{0.3} & 13.5 & 1315 & 2.76 & 9.64 & 1.18 \\
& 16.5 & 863 & 3.40 & 4.40 & 1.12 \\
& 20.0 & 662 & 3.89 & 3.89 & 1.05 \\
\hline \multirow{3}{*}{0.5} & 13.5 & 2415 & 2.03 & 15.34 & 1.28 \\
& 16.5 & 1613 & 2.49 & 6.59 & 1.19 \\
& 20.0 & 1190 & 2.90 & 4.13 & 1.16 \\
\hline \multirow{3}{*}{1.0} & 13.5 & 4419 & 1.50 & 25.32 & 1.36 \\
& 16.5 & 2967 & 1.84 & 12.14 & 1.25 \\
& 20.0 & 2235 & 2.12 & 8.03 & 1.21 \\
\hline \multirow{3}{*}{2.0} & 13.5 & 7438 & 1.16 & 39.49 & 1.42 \\
& 16.5 & 5041 & 1.41 & 17.23 & 1.28 \\
& 20.0 & 3826 & 1.62 & 13.55 & 1.25 \\
\hline \hline
\end{tabular}

Table 15: Neutrino raw data in $12-22 \mathrm{GeV}$ for different flux samples. 


\begin{tabular}{|c|c|c|c|c|c|}
\hline \hline$\nu \operatorname{cut}(\mathrm{GeV})$ & $\mathrm{E}(\mathrm{GeV})$ & $F^{\nu(\bar{\nu})}(E)$ & stat $(\%)$ & $B_{\Phi}^{\nu(\bar{\nu})}(E)$ & $A_{\phi}$ \\
\hline 0.3 & 13.5 & 247 & 6.36 & 0.91 & 1.04 \\
& 16.5 & 147 & 8.25 & 0.69 & 0.94 \\
& 20.0 & 110 & 9.53 & 0.91 & 0.96 \\
\hline 0.5 & 13.5 & 385 & 5.10 & 2.17 & 1.21 \\
& 16.5 & 224 & 6.68 & 1.29 & 1.09 \\
& 20.0 & 159 & 7.93 & 1.70 & 1.12 \\
\hline \multirow{2}{*}{1.0} & 13.5 & 636 & 3.97 & 4.69 & 1.33 \\
& 16.5 & 373 & 5.18 & 2.64 & 1.18 \\
& 20.0 & 260 & 6.20 & 3.47 & 1.19 \\
\hline \multirow{2}{*}{2.0} & 13.5 & 1033 & 3.11 & 8.85 & 1.42 \\
& 16.5 & 595 & 4.10 & 5.56 & 1.23 \\
& 20.0 & 427 & 4.84 & 5.00 & 1.23 \\
\hline
\end{tabular}

Table 16: Antineutrino raw data in $12-22 \mathrm{GeV}$ for different flux samples. 


\begin{tabular}{|c|c|c|c|c|}
\hline & \multicolumn{2}{|c|}{ Neutrino } & \multicolumn{2}{c|}{ Antineutrino } \\
\hline $\operatorname{Bin}(\mathrm{GeV})$ & $E_{\mu}>1.8 \mathrm{GeV}$ & $+\theta_{\mu \max }<0.35$ rads & $E_{\mu}>1.8 \mathrm{GeV}$ & $+\theta_{\mu \max }<0.35 \mathrm{rads}$ \\
\hline $2-3$ & $39.7 \%$ & $81.6 \%$ & $59.6 \%$ & $89.1 \%$ \\
$3-4$ & $60.9 \%$ & $82.9 \%$ & $85.3 \%$ & $90.7 \%$ \\
$4-5$ & $68.4 \%$ & $83.9 \%$ & $91.3 \%$ & $92.4 \%$ \\
$5-7$ & $75.8 \%$ & $84.6 \%$ & $94.6 \%$ & $94.5 \%$ \\
$7-9$ & $81.8 \%$ & $86.1 \%$ & $96.3 \%$ & $96.6 \%$ \\
$9-12$ & $85.9 \%$ & $87.9 \%$ & $96.8 \%$ & $97.4 \%$ \\
$12-15$ & $89.1 \%$ & $89.6 \%$ & $97.4 \%$ & $98.4 \%$ \\
$15-18$ & $90.9 \%$ & $91.2 \%$ & $97.8 \%$ & $98.8 \%$ \\
20.0 & $92.6 \%$ & $92.3 \%$ & $97.9 \%$ & $99.0 \%$ \\
\hline
\end{tabular}

Table 17: Effect of kinematic cuts. Column 2 and 4 show the percentage of all generated events passing the $E_{\mu}>1.8 \mathrm{GeV}$ cut for neutrino and antineutrino, respectively. Column 3 and 5 show the percentage of generated events with $E_{\mu}>1.8 \mathrm{GeV}$ cut passing the $\theta_{\mu \max }<0.35$ rads cut for neutrino and antineutrino, respectively.

\begin{tabular}{cccccccc}
\hline \hline Component & $\mathrm{H}$ & $\mathrm{C}$ & $\mathrm{O}$ & $\mathrm{Al}$ & $\mathrm{Si}$ & $\mathrm{Cl}$ & $\mathrm{Ti}$ \\
\hline planes & $8.18 \%$ & $88.5 \%$ & $2.5 \%$ & $0.07 \%$ & $0.07 \%$ & $0.20 \%$ & $0.47 \%$ \\
\hline \hline
\end{tabular}

Table 18: Composition of scintillator planes, by mass percentage. 


\begin{tabular}{|c|c|c|c|c|c|}
\hline \hline $\mathrm{E}$ & $A_{C C}^{K I N}$ & $S^{\nu(\bar{\nu})}\left(\nu_{0}, E\right)$ & $I_{i s o}^{\nu(\bar{\nu})}\left(\nu_{0}, E\right)$ & $H_{i s o}^{\nu(\bar{\nu})}\left(\nu_{o}\right)$ & stat \\
\hline 2.5 & 3.094 & 1.096 & 0.954 & 3.83 & 0.091 \\
\hline 3.5 & 1.981 & 1.040 & 0.982 & & \\
4.5 & 1.746 & 1.032 & 0.983 & 1.96 & 0.035 \\
6 & 1.559 & 1.023 & 0.984 & & \\
\hline 8 & 1.423 & 1.007 & 0.998 & 1.02 & 0.014 \\
10.5 & 1.326 & 1.005 & 0.998 & & \\
\hline 13.5 & 1.253 & 0.995 & 0.999 & & \\
16.5 & 1.207 & 0.992 & 0.999 & 0.574 & 0.006 \\
20 & 1.171 & 0.995 & 0.999 & & \\
\hline \hline
\end{tabular}

Table 19: Neutrino cross section model dependent corrections computing using GENIE hybrid.

\begin{tabular}{|c|c|c|c|c|c|}
\hline \hline $\mathrm{E}$ & $A_{C C}^{K I N}$ & $S^{\nu(\bar{\nu})}\left(\nu_{0}, E\right)$ & stat & $I_{i s o}^{\nu(\bar{\nu})}\left(\nu_{0}, E\right)$ & stat \\
\hline 2.5 & 2.904 & 1.12 & 0.0095 & 0.955 & 0.0015 \\
\hline 3.5 & 2.099 & 1.068 & 0.0072 & 0.981 & 0.0013 \\
4.5 & 1.820 & 1.053 & 0.0072 & 0.983 & 0.0015 \\
6 & 1.607 & 1.039 & 0.0073 & 0.985 & 0.0017 \\
\hline 8 & 1.453 & 1.016 & 0.0055 & 1.002 & 0.0015 \\
10.5 & 1.344 & 1.011 & 0.0056 & 1.002 & 0.0017 \\
\hline 13.5 & 1.267 & 0.996 & 0.0043 & 0.999 & 0.0020 \\
16.5 & 1.218 & 0.996 & 0.0043 & 1.003 & 0.0016 \\
20 & 1.180 & 0.995 & 0.0043 & 1.003 & 0.0018 \\
\hline \hline
\end{tabular}

Table 20: Neutrino cross section model dependent corrections computed using NuWro. 


\begin{tabular}{|c|c|c|c|}
\hline \hline $\mathrm{E}$ & $A_{C C}^{K I N}$ & $S^{\nu(\bar{\nu})}\left(\nu_{0}, E\right)$ & $I_{i s o}^{\nu(\bar{\nu})}\left(\nu_{0}, E\right)$ \\
\hline 2.5 & 1.883 & 0.801 & 1.042 \\
\hline 3.5 & 1.293 & 0.809 & 1.016 \\
4.5 & 1.185 & 0.850 & 1.016 \\
6 & 1.118 & 0.884 & 1.016 \\
\hline 8 & 1.076 & 0.869 & 1.005 \\
10.5 & 1.060 & 0.899 & 1.005 \\
\hline 13.5 & 1.044 & 0.875 & 1.004 \\
16.5 & 1.035 & 0.893 & 1.004 \\
20 & 1.032 & 0.912 & 1.004 \\
\hline
\end{tabular}

Table 21: Antineutrino cross section model dependent corrections computed using GENIE hybrid.

\begin{tabular}{|c|c|c|c|c|c|}
\hline \hline $\mathrm{E}$ & $A_{C C}^{K I N}$ & $S^{\nu(\bar{\nu})}\left(\nu_{0}, E\right)$ & stat & $I_{i s o}^{\nu(\bar{\nu})}\left(\nu_{0}, E\right)$ & stat \\
\hline 2.5 & 1.756 & 0.798 & 0.0068 & 1.041 & 0.0010 \\
\hline 3.5 & 1.346 & 0.804 & 0.0052 & 1.016 & 0.0008 \\
4.5 & 1.219 & 0.847 & 0.0052 & 1.015 & 0.0010 \\
6 & 1.137 & 0.883 & 0.0052 & 1.017 & 0.0011 \\
\hline 8 & 1.087 & 0.872 & 0.0040 & 1.001 & 0.0010 \\
10.5 & 1.059 & 0.903 & 0.0040 & 1.000 & 0.0011 \\
\hline 13.5 & 1.042 & 0.875 & 0.0030 & 0.998 & 0.0009 \\
16.5 & 1.033 & 0.893 & 0.0030 & 1.002 & 0.0011 \\
20 & 1.027 & 0.913 & 0.0031 & 1.000 & 0.0012 \\
\hline \hline
\end{tabular}

Table 22: Antineutrino cross section model dependent corrections computed using NuWro. 

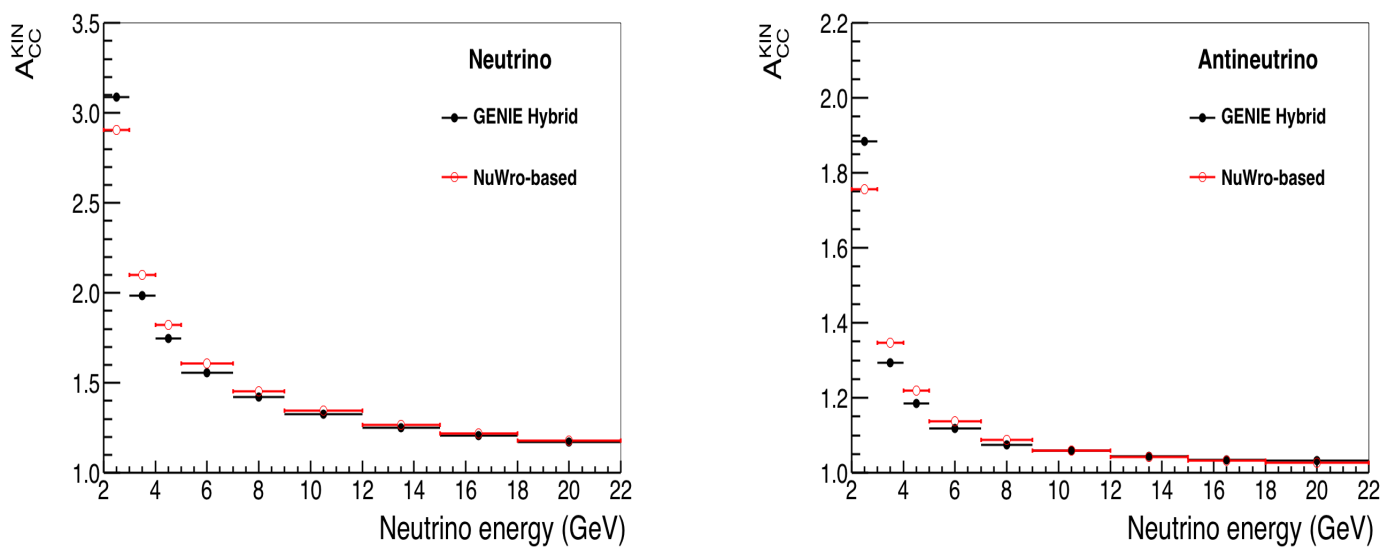

Figure 52: Kinematic corrections for FHC neutrino(left) and RHC antineutrino(right).

\begin{tabular}{cc}
\hline \hline$\nu<\nu_{0}$ cut & $G_{\text {corr }}\left(\nu_{0}\right)$ \\
\hline$\nu<0.3$ & 1.000 \\
$\nu<0.5$ & 1.004 \\
$\nu<1$ & 1.015 \\
$\nu<2$ & 1.026 \\
\hline \hline
\end{tabular}

Table 23: GENIE-based $G_{c o r r}\left(\nu_{0}\right)$ correction for different $\nu$ cut. 

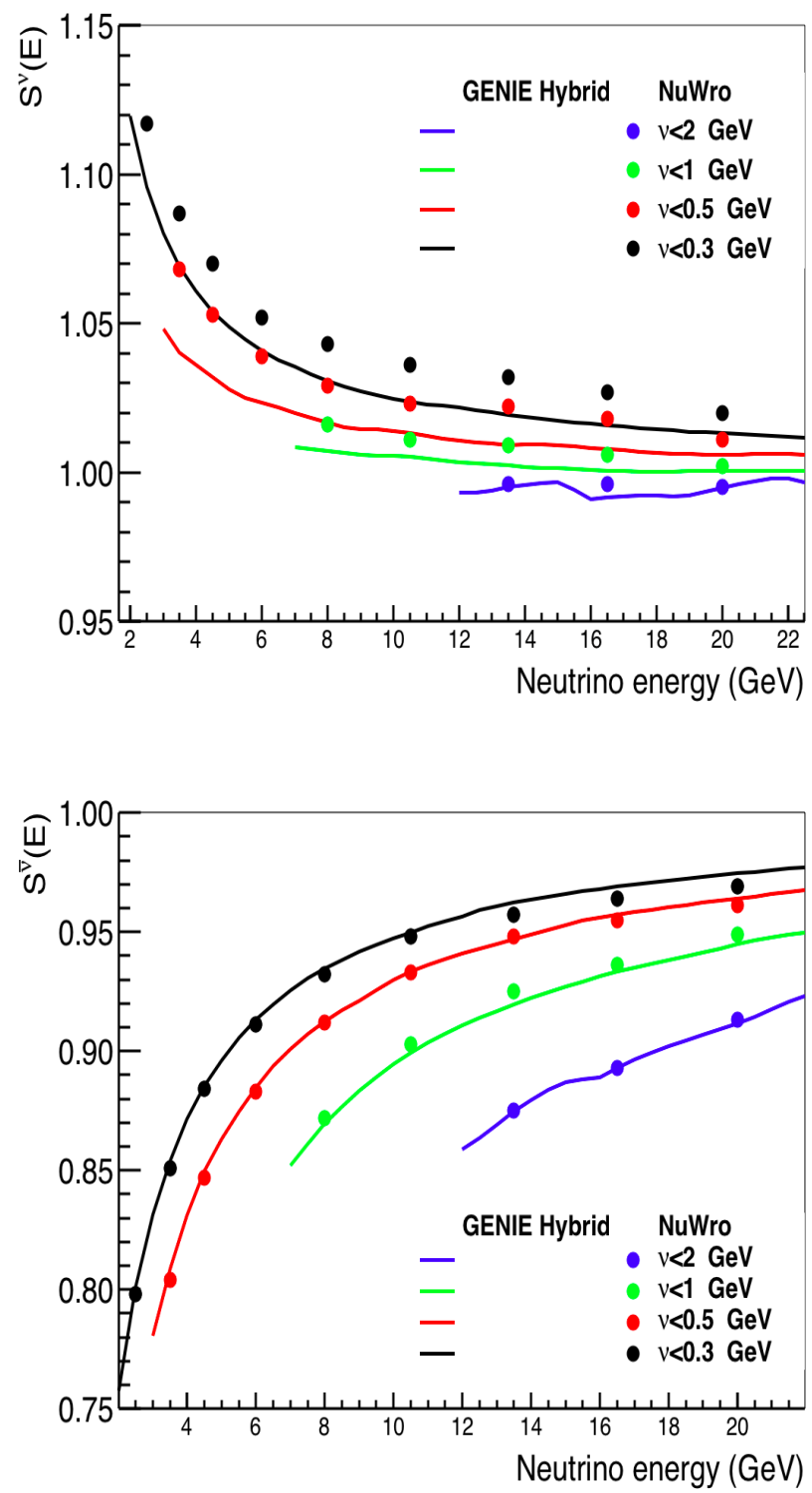

Figure 53: GENIE-based (solid curves) and NuWro-based (dots) low- $\nu$ correction for neutrinos (upper) and antineutrinos (lower). Both versions have similar shape as a function of neutrino energy, however, the NuWro-based version is always above the GENIE-based value. 

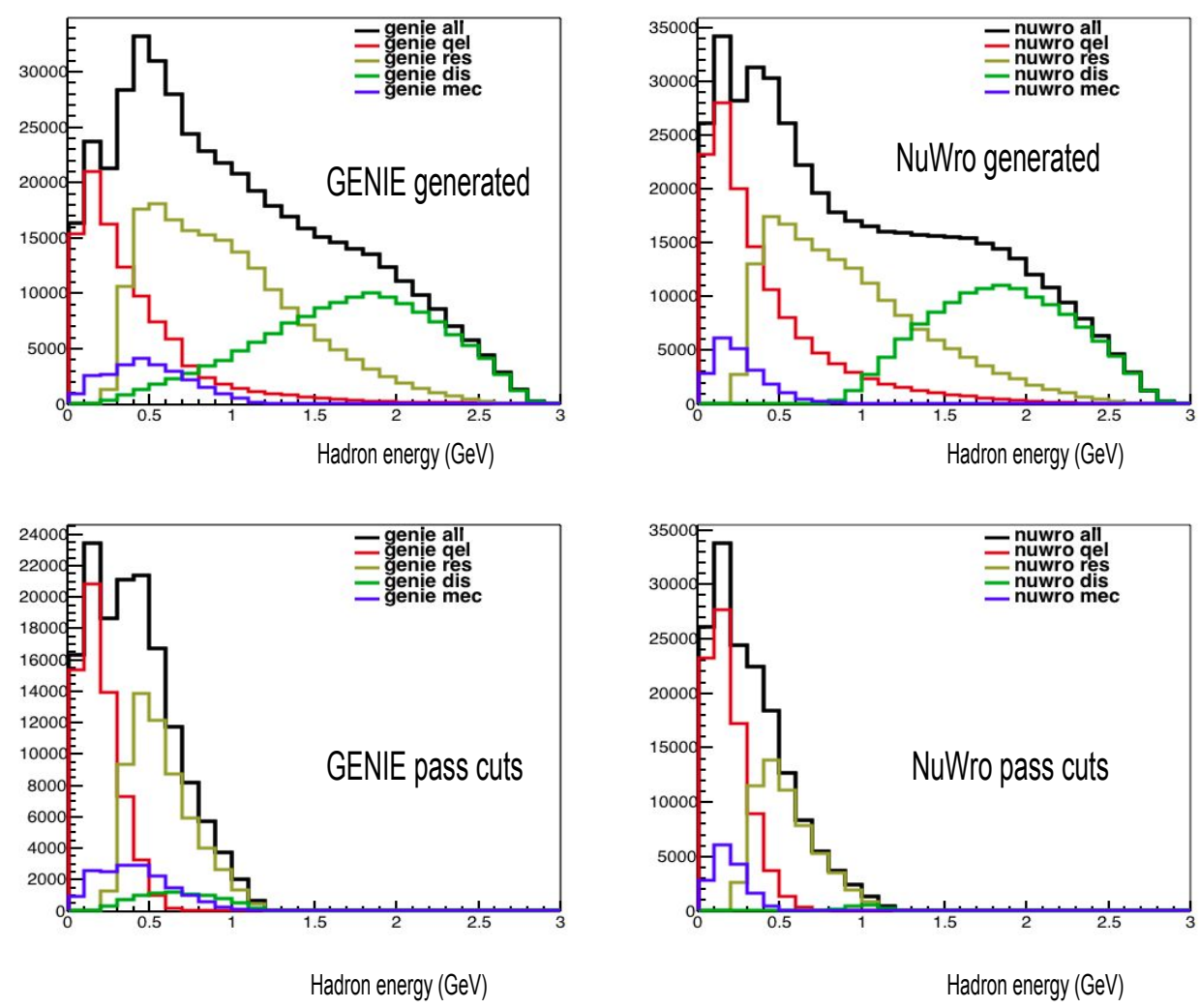

Figure 54: Hadron energy distributions and their breakdown in the energy region $2<E_{\nu}<3 \mathrm{GeV}$ of simulated GENIE (left) and NuWro (right) samples for neutrino. Plots on top show all generated events, while the bottom ones show events which pass kinematic cuts. 

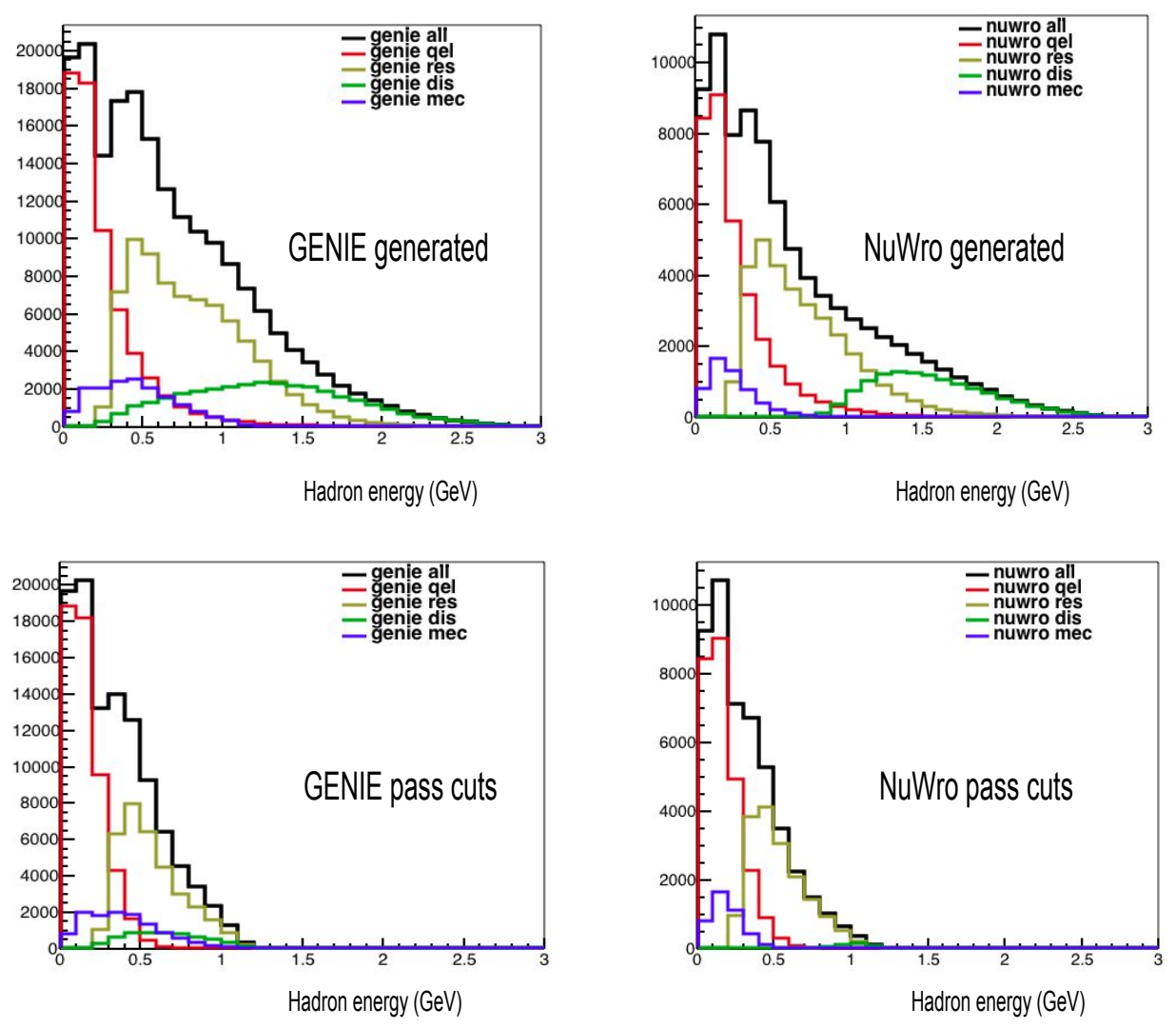

Figure 55: Hadron energy distributions and their breakdown in the energy region $2<E_{\nu}<3 \mathrm{GeV}$ of simulated GENIE (left) and NuWro (right) samples for antineutrino. Plots on top show all generated events, while the bottom ones show events which pass kinematic cuts. 


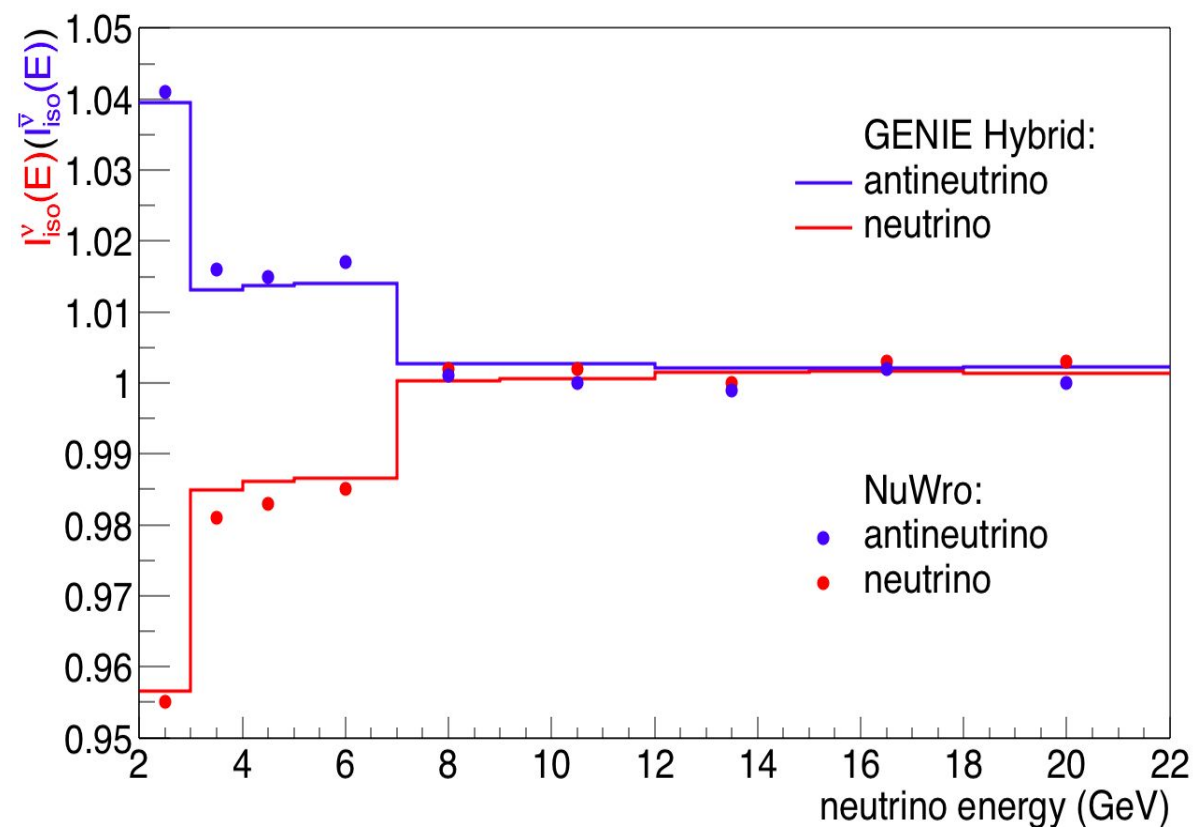

Figure 56: GENIE-based and NuWro-based isoscalar corrections for neutrinos and antineutrinos. 


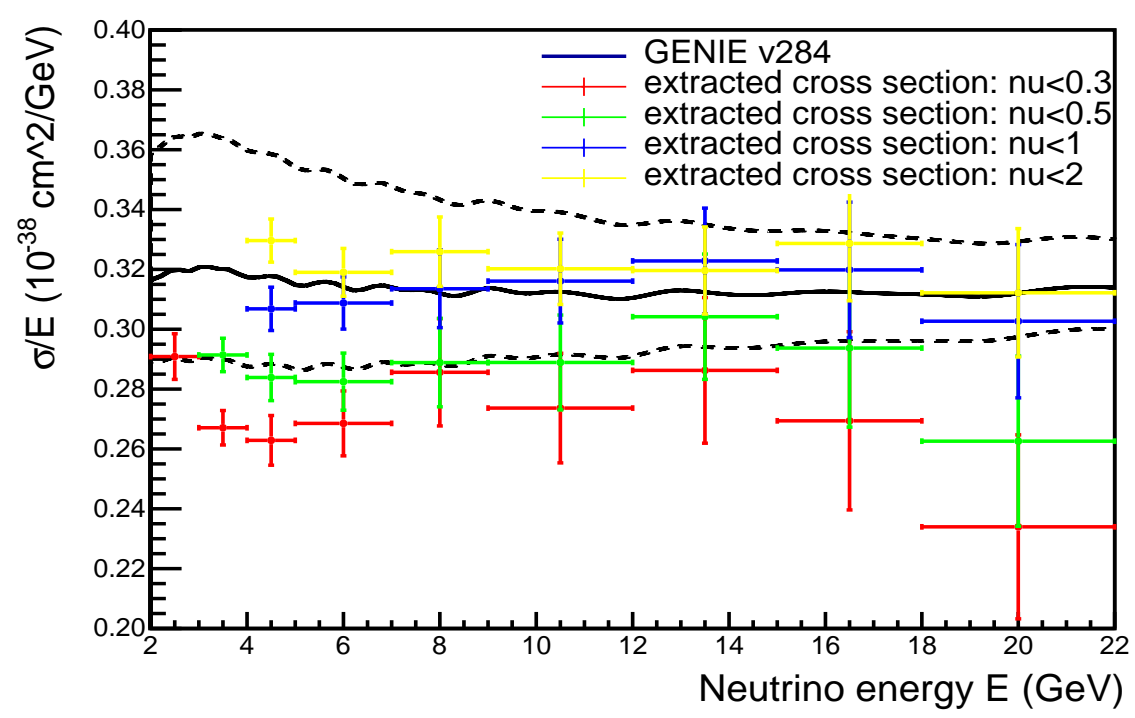

Figure 57: Extracted antineutrino cross sections using different $\nu$ cuts. Error bars are statistical errors only.

\begin{tabular}{ccc}
\hline \hline$\nu$ cut & $\alpha\left(\nu_{0}\right)$ & Statistical error \\
\hline 0.3 & 1.126 & $5.95 \%$ \\
0.5 & 1.056 & $4.83 \%$ \\
1 & 1.005 & $3.88 \%$ \\
\hline \hline
\end{tabular}

Table 24: $\alpha\left(\nu_{0}\right)$ and its statistical uncertainty. 


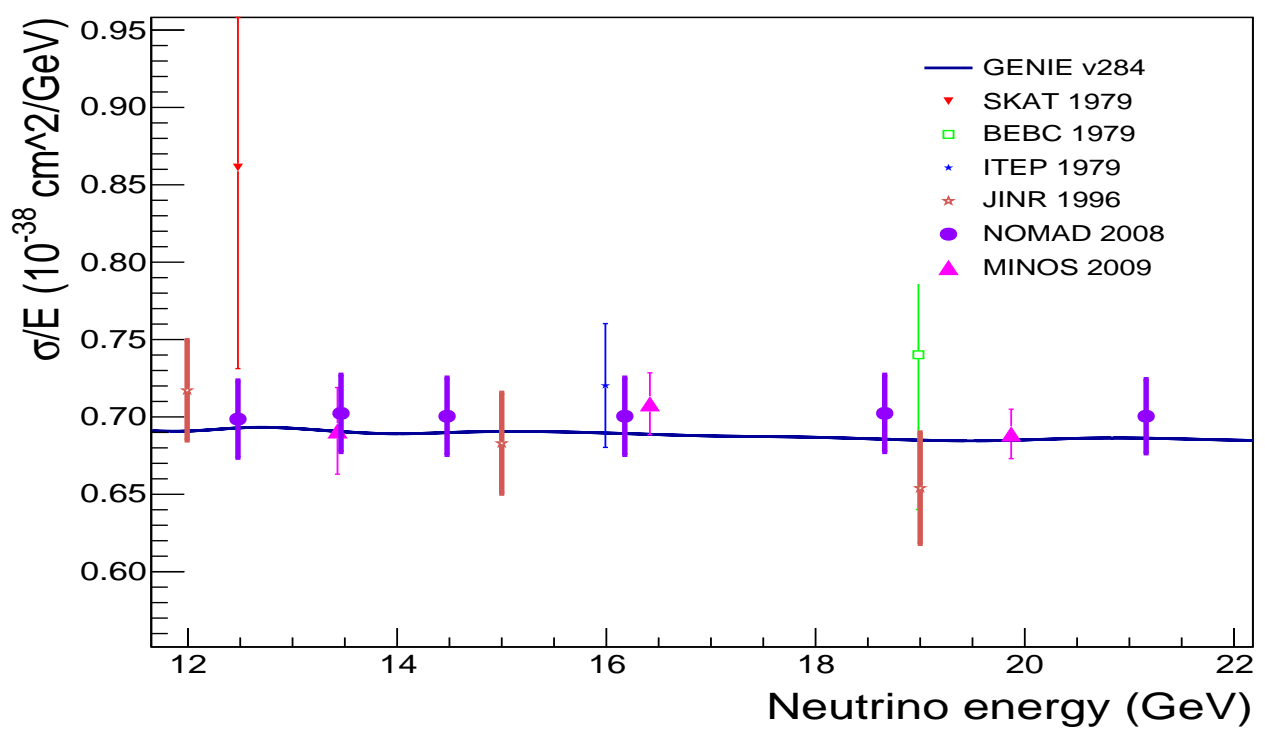

Figure 58: World measurements of CC inclusive neutrino cross section between 12 and $22 \mathrm{GeV}$.

\begin{tabular}{cccc}
\hline \hline Experiment & Target & Energy bin center $(\mathrm{GeV})$ & $\sigma / E\left(\times 10^{-38} \mathrm{~cm}^{2} / \mathrm{GeV}\right)$ \\
\hline NOMAD(2007) & Carbon & 12.5 & $0.697 \pm 0.025$ \\
& & 13.5 & $0.700 \pm 0.025$ \\
& & 14.5 & $0.698 \pm 0.025$ \\
& & 18.7 & $0.698 \pm 0.025$ \\
& & 21.2 & $0.700 \pm 0.025$ \\
MINOS(2009) & Iron & 13.43 & $0.699 \pm 0.024$ \\
& & 16.42 & $0.691 \pm 0.028$ \\
& & 19.87 & $0.708 \pm 0.020$ \\
\hline \hline
\end{tabular}

Table 25: World isoscalar neutrino cross section data at $12-22 \mathrm{GeV}$. 


\subsection{SYSTEMATIC UNCERTAINTY}

In this chapter, we present the evaluation of systematic uncertainties of measured quantities. These include normalization, reconstruction related (including muon energy scale and hadron energy scale, shower cleaning correction and background), and cross section model uncertainties. GENIE 2.8.4 is used to simulate event samples with our full detector simulation and to correct for reconstruction effects. The reweightable GENIE cross section model and FSI uncertainties are evaluated from default GENIE 2.8.4. GENIE-Hybrid model specific uncertainties are evaluated separately as described below.

\subsection{NORMALIZATION}

There are three pieces in normalization uncertainty, uncertainty on the external normalization of data samples, uncertainty of $G_{c o r r}$ and statistical error of our data in normalization region (which propagates to all energies through the normalization procedure). External normalization uncertainty is $3.58 \%$ from NOMAD measurement described in Sec. 6.5.2. This enters into cross section and flux measurements, however, it cancels for the ratio, $r$. An uncertainty due to the computed $G_{c o r r}$ is evaluated by calculating the fractional difference between the nominal $G_{c o r r}$ corrections (Tab. 23) and the shifted values with GENIE cross section model parameters shifted by $1 \sigma$ (discussed in Sec. 7.7). This uncertainty enters into antineutrino flux and cross section, as well as the ratio, $r$. Tab. 26 shows a summary of sources of normalization uncertainty. The statistical error introduced by the normalization procedure is included in the total statistical error of measured quantities and will be shown in Sec. 8.1. 


\begin{tabular}{cccccc}
\hline \hline $\begin{array}{c}E_{\nu} \\
(\mathrm{GeV})\end{array}$ & $\begin{array}{c}\nu \text { cut } \\
(\mathrm{GeV})\end{array}$ & $\begin{array}{c}\text { External } \\
\text { uncertainty }\end{array}$ & $\begin{array}{c}G_{\text {corr }}\left(\nu_{0}\right) \\
\text { uncertainty }\end{array}$ & $\begin{array}{c}\text { Stat. err. of } \\
\text { unnormalized } \sigma_{C C}^{\nu}(17 \mathrm{GeV})\end{array}$ & $\begin{array}{c}\alpha\left(\nu_{0}\right) \\
\text { uncertainty }\end{array}$ \\
\hline $2-3$ & 0.3 & $3.58 \%$ & $0 \%$ & $2.38 \%$ & $5.95 \%$ \\
$3-7$ & 0.5 & $3.58 \%$ & $0.12 \%$ & $1.79 \%$ & $4.83 \%$ \\
$7-12$ & 1.0 & $3.58 \%$ & $0.26 \%$ & $1.37 \%$ & $3.88 \%$ \\
$12-22$ & 2.0 & $3.58 \%$ & $0.43 \%$ & $1.05 \%$ & NA \\
\hline \hline
\end{tabular}

Table 26: Summary of normalization related uncertainties.

\subsection{RECONSTRUCTION}

Reconstruction uncertainty includes muon energy scale, hadron energy scale, shower cleaning correction and background modeling.

\subsubsection{Muon Energy Scale}

There are three sources of uncertainty considered for muon energy reconstruction, which are independent and added in quadrature. Tab. 27 shows the sources which include MINOS range and curvature measurement and uncertainties of energy loss in MINERvA. MINOS range uncertainty is present for all reconstructed muon tracks, while MINOS curvature uncertainty is only relevant to muon tracks which are reconstructed by curvature.

These uncertainties are evaluated with MC by simultaneously shifting cross section and flux samples. The $1 \sigma$ parameter uncertainties are shown in Tab. 27. The analysis is repeated in 100 universes $^{1}$. For each universe, four components of muon energy scale are shifted at the same time and the effect is added in quadrature. An error band is obtained from the RMS band in resulting measured quantities.

\footnotetext{
${ }^{1}$ For a certain systematic, each "universe" represents a deviation from the nominal value. Those deviations are expected to form a Gaussian distribution.
} 


\begin{tabular}{cc}
\hline \hline Error Source & Error \\
\hline MINOS Range & $2 . \%$ \\
\hline MINOS Curvature $\left(p_{\mu}<1 G e V\right)$ & $2.5 \%$ \\
MINOS Curvature $\left(p_{\mu}>1 G e V\right)$ & $0.6 \%$ \\
\hline MINERvA $\frac{d E}{d x}$ (scintillator) & $30 \mathrm{MeV}$ \\
\hline MINERvA mass (scintillator) & $11 \mathrm{MeV}$ \\
\hline \hline
\end{tabular}

Table 27: Components of muon energy scale.

\subsubsection{Hadron Energy Scale}

From MINER $\nu$ A test beam studies [50], we obtain an uncertainty for each type of particle depositing energy in the MINER $\nu \mathrm{A}$ detector as listed in Tab. 28. The muon response comes from the uncertainty of determining minimum-ionizing energy unit (MEU), which is due to the detector mass model, and also the uncertainty of Bethe-Bloche process used to simulate the deposited energy. The electromagnetic response is obtained by studying the difference between data and simluation of the energy spectrum of Michel electrons (produced in the decay of an antimuon). The $1 \sigma$ uncertainty is the average for each particle type weighted by the fraction of energy contributed by that particle. The procedure of evaluating hadron energy scale is similar as for the muon energy scale.

The hadron energy scale uncertainties for neutrino and antineutrino cross sections are shown in Fig. 59 and Fig. 60 and Fig. 61. There is partial cancellation between neutrino and antineutrino cross section uncertainties in the ratio, $r$. Uncertainties from all components are shown in Tab. 29, Tab. 30, and Tab. 31 for neutrino, antineutrino cross sections and the ratio, $r$, respectively. 


\begin{tabular}{cc}
\hline \hline Energy Source & Error \\
\hline Proton & $3.5 \%$ \\
Neutron $(\mathrm{KE}<50 \mathrm{MeV})$ & $25 \%$ \\
Neutron $(50<\mathrm{KE}<150 \mathrm{MeV})$ & $10 \%$ \\
Neutron $(\mathrm{KE}>150 \mathrm{MeV})$ & $20 \%$ \\
Muon & $2.4 \%$ \\
$\gamma, \pi^{0}, e^{ \pm}$ & $3 \%$ \\
$\pi^{ \pm}$, Kaon & $5 \%$ \\
Cross talk & $20 \%$ \\
Other & $20 \%$ \\
\hline \hline
\end{tabular}

Table 28: Hadron energy scale uncertainty components.

\begin{tabular}{|c|c|c|c|c|c|c|c|c|c|}
\hline \hline Source & $\begin{array}{c}2.5 \\
\mathrm{GeV}\end{array}$ & $\begin{array}{c}3.5 \\
\mathrm{GeV}\end{array}$ & $\begin{array}{c}4.5 \\
\mathrm{GeV}\end{array}$ & $\begin{array}{c}6 \\
\mathrm{GeV}\end{array}$ & $\begin{array}{c}8 \\
\mathrm{GeV}\end{array}$ & $\begin{array}{c}10.5 \\
\mathrm{GeV}\end{array}$ & $\begin{array}{c}13.5 \\
\mathrm{GeV}\end{array}$ & $\begin{array}{c}16.5 \\
\mathrm{GeV}\end{array}$ & $\begin{array}{c}20 \\
\mathrm{GeV}\end{array}$ \\
\hline pion & 0.0008 & 0.0021 & 0.0050 & 0.0028 & 0.0013 & 0.0000 & 0.0031 & 0.0047 & 0.0083 \\
proton & 0.0068 & 0.0034 & 0.0010 & 0.0005 & 0.0005 & 0.0000 & 0.0015 & 0.0013 & 0.0016 \\
high n & 0.0031 & 0.0016 & 0.0011 & 0.0006 & 0.0007 & 0.0000 & 0.0028 & 0.0034 & 0.0065 \\
medium n & 0.0012 & 0.0007 & 0.0005 & 0.0002 & 0.0002 & 0.0000 & 0.0002 & 0.0001 & 0.0002 \\
low n & 0.0008 & 0.0005 & 0.0002 & 0.0002 & 0.0002 & 0.0000 & 0.0002 & 0.0002 & 0.0002 \\
em & 0.0012 & 0.0008 & 0.0011 & 0.0007 & 0.0006 & 0.0000 & 0.0004 & 0.0015 & 0.0029 \\
muon & 0.0122 & 0.0069 & 0.0025 & 0.0016 & 0.0007 & 0.0000 & 0.0011 & 0.0007 & 0.0013 \\
other & 0.0021 & 0.0019 & 0.0033 & 0.0031 & 0.0014 & 0.0000 & 0.0023 & 0.0021 & 0.0040 \\
xtalk & 0.0127 & 0.0067 & 0.0016 & 0.0006 & 0.0009 & 0.0000 & 0.0033 & 0.0047 & 0.0061 \\
\hline \hline
\end{tabular}

Table 29: Breakdown of hadron energy scale uncertainty for neutrino cross section. 


\begin{tabular}{|c|c|c|c|c|c|c|c|c|c|}
\hline \hline Source & $\begin{array}{c}2.5 \\
\mathrm{GeV}\end{array}$ & $\begin{array}{c}3.5 \\
\mathrm{GeV}\end{array}$ & $\begin{array}{c}4.5 \\
\mathrm{GeV}\end{array}$ & $\begin{array}{c}6 \\
\mathrm{GeV}\end{array}$ & $\begin{array}{c}8 \\
\mathrm{GeV}\end{array}$ & $\begin{array}{c}10.5 \\
\mathrm{GeV}\end{array}$ & $\begin{array}{c}13.5 \\
\mathrm{GeV}\end{array}$ & $\begin{array}{c}16.5 \\
\mathrm{GeV}\end{array}$ & $\begin{array}{c}20 \\
\mathrm{GeV}\end{array}$ \\
\hline pion & 0.0011 & 0.0017 & 0.0059 & 0.0043 & 0.0038 & 0.0029 & 0.0048 & 0.0083 & 0.0084 \\
proton & 0.0084 & 0.0074 & 0.0049 & 0.0018 & 0.0016 & 0.0012 & 0.0008 & 0.0016 & 0.0011 \\
highn & 0.0027 & 0.0018 & 0.0073 & 0.0047 & 0.0068 & 0.0073 & 0.0058 & 0.0080 & 0.0082 \\
medium n & 0.0009 & 0.0002 & 0.0004 & 0.0002 & 0.0005 & 0.0004 & 0.0003 & 0.0002 & 0.0003 \\
low n & 0.0006 & 0.0002 & 0.0007 & 0.0002 & 0.0003 & 0.0003 & 0.0002 & 0.0001 & 0.0003 \\
em & 0.0000 & 0.0002 & 0.0001 & 0.0004 & 0.0016 & 0.0009 & 0.0016 & 0.0012 & 0.0013 \\
muon & 0.0135 & 0.0083 & 0.0035 & 0.0008 & 0.0012 & 0.0008 & 0.0007 & 0.0018 & 0.0014 \\
other & 0.0021 & 0.0024 & 0.0039 & 0.0042 & 0.0031 & 0.0030 & 0.0031 & 0.0051 & 0.0049 \\
xtalk & 0.0149 & 0.0096 & 0.0034 & 0.0009 & 0.0012 & 0.0023 & 0.0027 & 0.0061 & 0.0048 \\
\hline \hline
\end{tabular}

Table 30: Breakdown of hadron energy scale uncertainty for antineutrino cross section.

\begin{tabular}{|c|c|c|c|c|c|c|c|c|c|}
\hline \hline Source & 2.5 & 3.5 & 4.5 & 6 & $\begin{array}{c}8 \\
\mathrm{GeV}\end{array}$ & $\mathrm{GeV}$ & $\mathrm{GeV}$ & $\mathrm{GeV}$ & $\mathrm{GeV}$ \\
$\mathrm{GeV}$ & $\begin{array}{c}13.5 \\
\mathrm{GeV}\end{array}$ & $\begin{array}{c}16.5 \\
\mathrm{GeV}\end{array}$ & $\begin{array}{c}20 \\
\mathrm{GeV}\end{array}$ \\
\hline pion & 0.0007 & 0.0005 & 0.0010 & 0.0016 & 0.0029 & 0.0029 & 0.0019 & 0.0043 & 0.0011 \\
proton & 0.0016 & 0.0040 & 0.0057 & 0.0018 & 0.0019 & 0.0012 & 0.0016 & 0.0005 & 0.0009 \\
high n & 0.0005 & 0.0034 & 0.0064 & 0.0046 & 0.0074 & 0.0073 & 0.0031 & 0.0047 & 0.0028 \\
medium n & 0.0003 & 0.0006 & 0.0008 & 0.0004 & 0.0007 & 0.0004 & 0.0003 & 0.0002 & 0.0004 \\
low n & 0.0003 & 0.0004 & 0.0007 & 0.0003 & 0.0004 & 0.0003 & 0.0002 & 0.0003 & 0.0002 \\
em & 0.0001 & 0.0002 & 0.0002 & 0.0004 & 0.0016 & 0.0009 & 0.0017 & 0.0013 & 0.0011 \\
muon & 0.0013 & 0.0014 & 0.0012 & 0.0010 & 0.0013 & 0.0008 & 0.0010 & 0.0014 & 0.0013 \\
other & 0.0001 & 0.0005 & 0.0006 & 0.0012 & 0.0018 & 0.0030 & 0.0010 & 0.0032 & 0.0012 \\
xtalk & 0.0022 & 0.0029 & 0.0037 & 0.0007 & 0.0008 & 0.0023 & 0.0010 & 0.0019 & 0.0026 \\
\hline \hline
\end{tabular}

Table 31: Breakdown of hadron energy scale uncertainty for the ratio, $r$. 


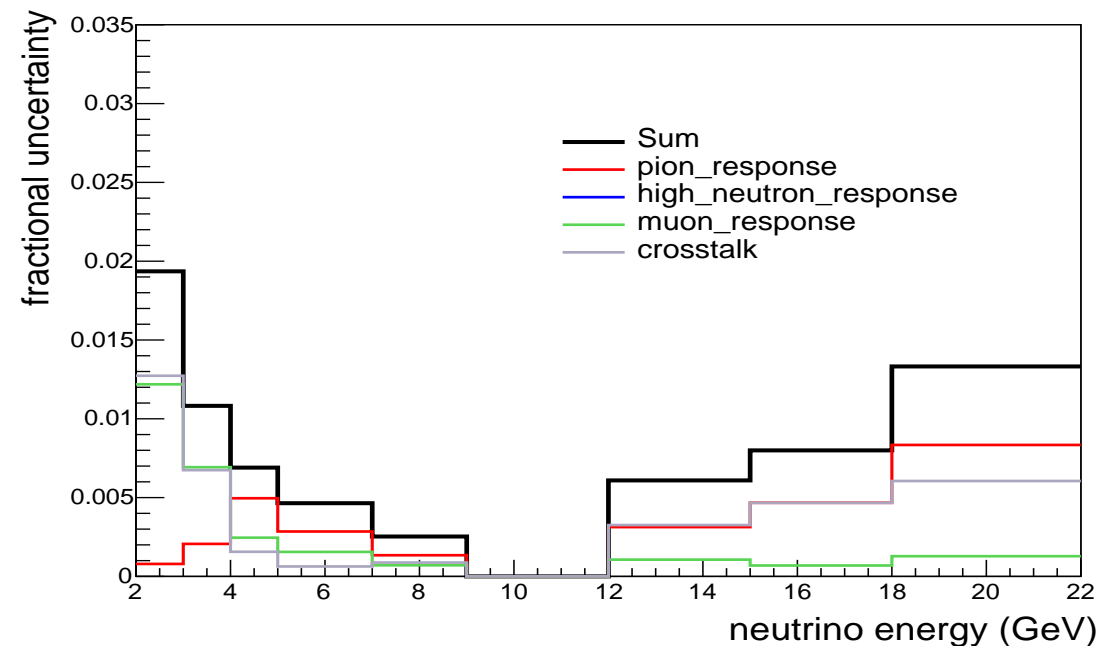

Figure 59: Breakdown of hadron energy scale uncertainties for neutrino cross section.

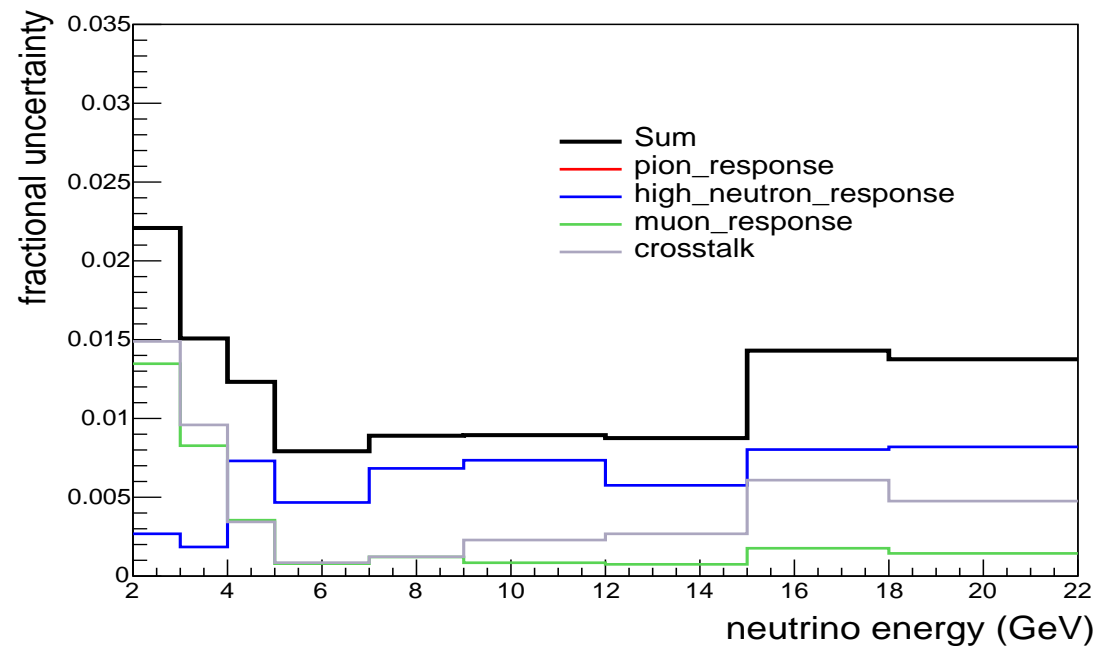

Figure 60: Breakdown of hadron energy scale uncertainties for antineutrino cross section. 


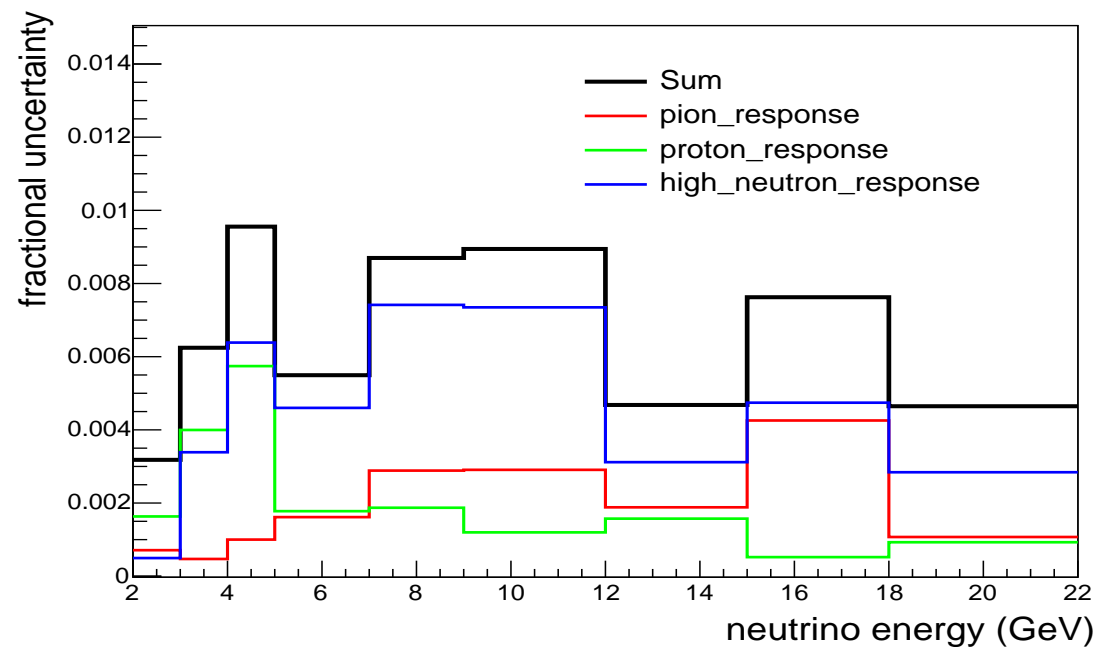

Figure 61: Breakdown of hadron energy scale uncertainties for cross section ratio $r$. 


\subsubsection{Shower Cleaning Correction}

We include an estimated uncertainty in the size of the shower cleaning correction (described in Sec. 4.6.1) in measured quantities. We assign $50 \%$ of the change of measured quantities with and without shower cleaning correction as an estimate of its uncertainty. Fig. 62 and Fig. 63 show the comparison with and without shower cleaning corrections. The largest effect on cross section is at the lowest neutrino energy $2-3 \mathrm{GeV}$ (about $1 \%$ for the neutrino and $2 \%$ for the antineutrino cross section in that bin).

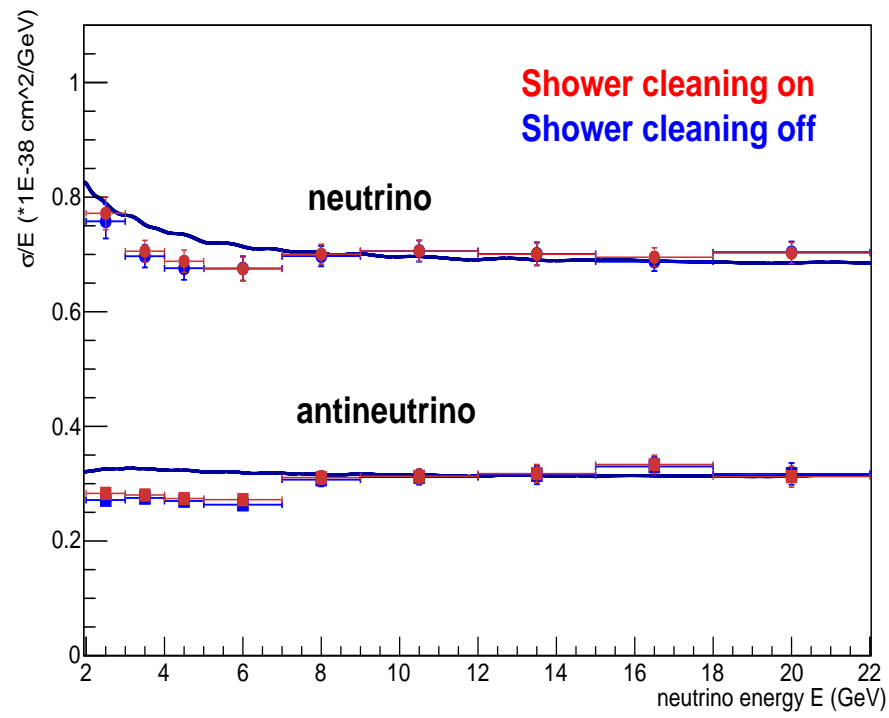

Figure 62: Effect of shower cleaning corrections on cross sections.

\subsubsection{Background}

The wrong-sign contamination in the selected antineutrino samples in RHC beam mode is obtained from MC simulation as shown in Fig. 49 (with nominal input flux). We include an uncertainty in this source of background in the antineutrino cross section and $r$ measurements.

To obtain an estimate of uncertainty from this background, a low- $\nu$ flux reweighting function for RHC neutrinos is extracted. We vary neutrino flux in FHC mode with the reweighting function 


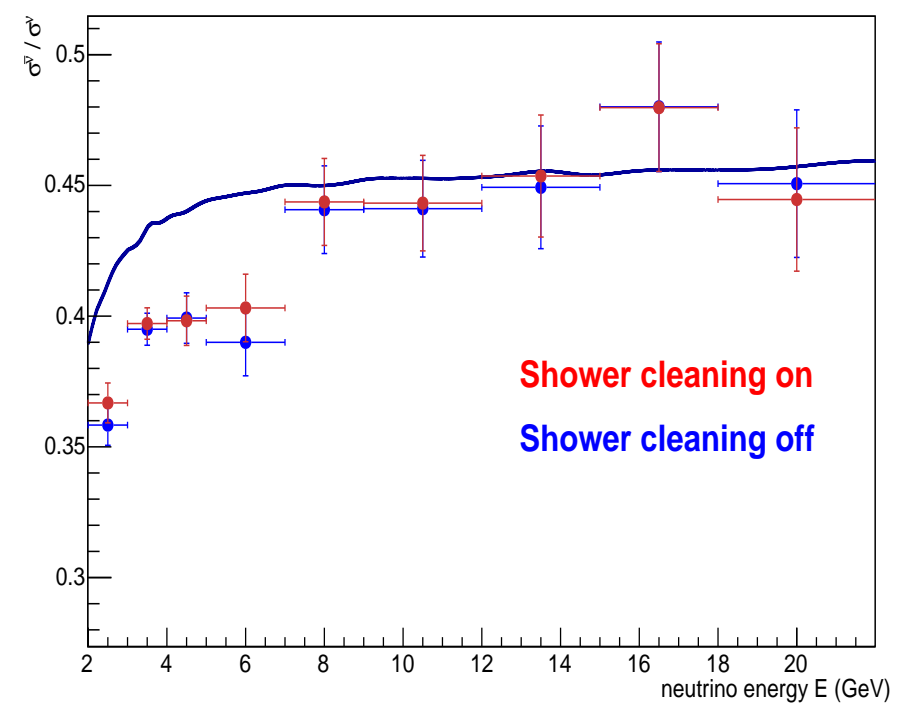

Figure 63: Effect of shower cleaning corrections on cross section ratio $r$.

shown in Fig. 64. The wrong-sign events (RHC neutrinos) are then reweighted and a new antineutrino cross section is extracted. The fractional difference between the nominal and reweighted antineutrino cross section (and $r$ ) is taken as the uncertainty. Because the contamination is always less than $4 \%$, this uncertainty is less than $1 \%$ on the cross section and ratio, $r$, everywhere.

\subsection{FINAL STATE INTERACTION UNCERTAINTIES}

Hadrons produced in neutrino scattering interactions may rescatter in the nuclear environment before they exit the nucleus, which modifies the observable distributions. The sources of FSI uncertainties in GENIE are shown in Tab. 32 and Tab. 36 [1].

For those in Tab. 32, they are evaluated by reweighting MC events. For each uncertainty in the table, cross section sample and flux samples are shifted $\pm 1 \sigma$, cross sections are re-exacted, the fractional difference from the nominal are assigned as the uncertainties. Each uncertainty in the table is evaluated independently and added in quadrature. Fig. 66, Fig. 67 and Fig. 68 show 


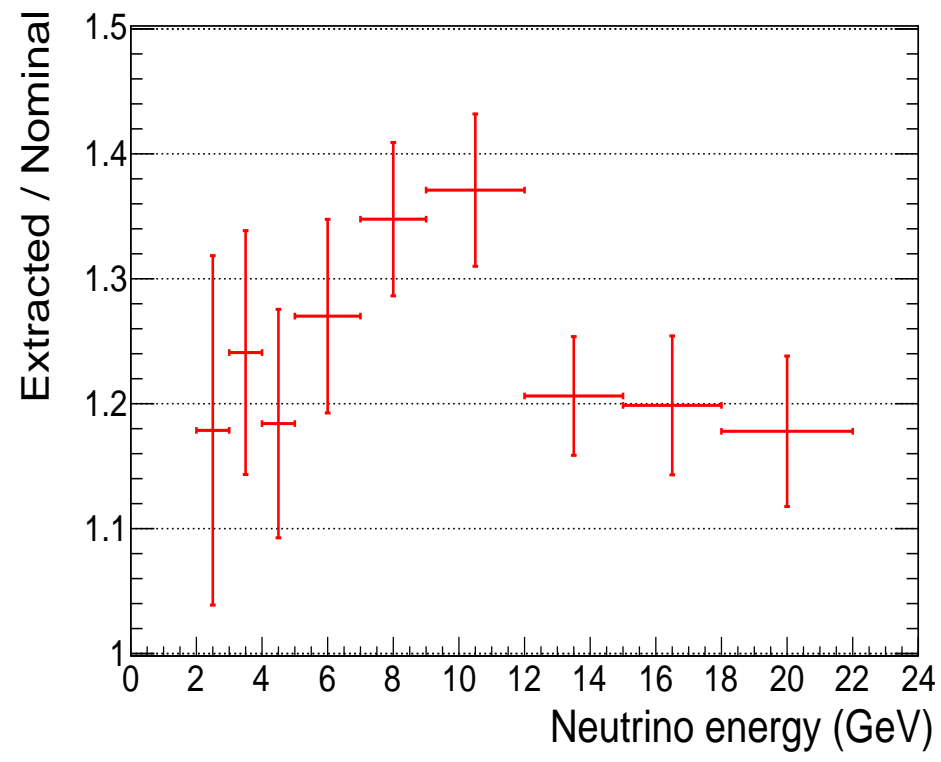

Figure 64: Low- $\nu$ flux reweighting function for RHC neutrinos.

the uncertainties from each uncertainty on neutrino, antineutrino cross sections and the ratio, $r$, respectively.

Uncertainties are shown in Tab. 36 which arise from the size of nuclear formation zone, and variation of AGKY model, are evaluated using another set of MC samples with those parameters each shifted by $1 \sigma$ are generated. However, due to poor MC statistics, we re-extract cross sections and $r$ with a coarser binning (one neutrino energy bin for each $\nu$ cut region). The ratios of the shifted quantities to the nominal are fitted to constants as shown in Fig. 65 and error bands are obtained from fitted values. Uncertainties of non-reweightable FSI are less than 1\%. These are then added to reweightable components in quadrature to obtain full FSI uncertainties. 

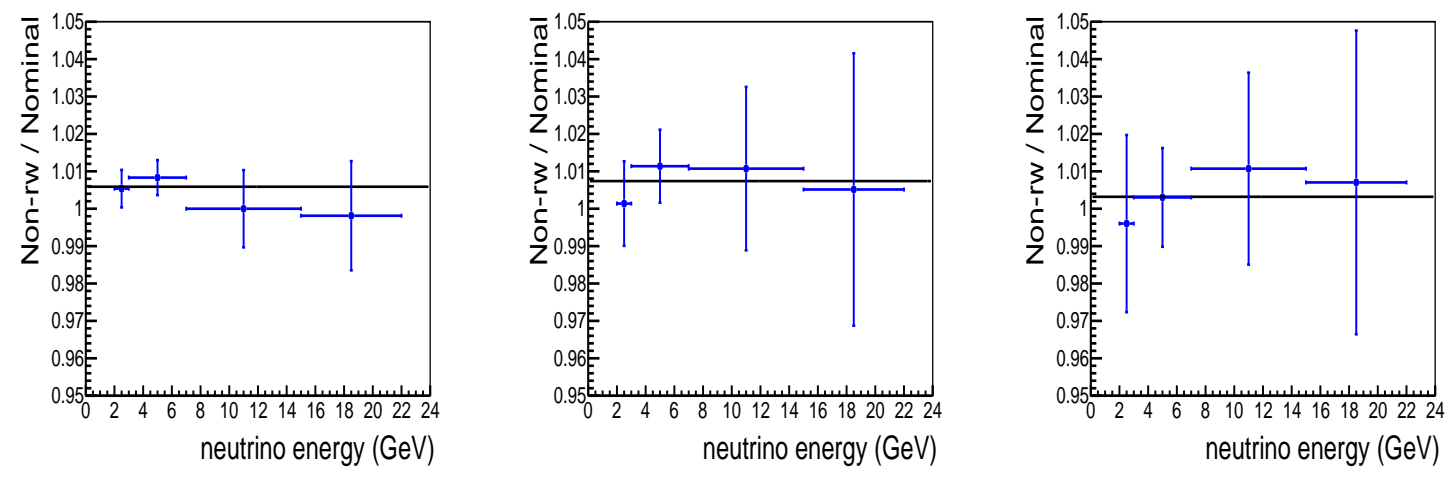

Figure 65: Shifted / nominal neutrino (left) and antineutrino (middle) cross sections and $r$ (right) due to non-reweightable FSI uncertainties. The black line in each figure is the constant fit of the data plots and is taken as the error of non-reweightable FSI model uncertainties. 


\begin{tabular}{ccc}
\hline \hline GENIE Knob name & Description & $1 \sigma$ \\
\hline MFP_N & mean free path for nucleons & $\pm 20 \%$ \\
FrCex_N & nucleon fates - charge exchange & $\pm 50 \%$ \\
FrElas_N & nucleon fates - elastic & $\pm 30 \%$ \\
Frinel_N & nucleon fates - inelastic & $\pm 40 \%$ \\
FrAbs_N & nucleon fates - absorption & $\pm 20 \%$ \\
FrPiProd_N & nucleon fates - pion production & $\pm 20 \%$ \\
MFP_pi & mean free path for pions & $\pm 20 \%$ \\
FrCEx_pi & pion fates - charge exchange & $\pm 50 \%$ \\
FrElas_pi & pion fates - elastic & $\pm 10 \%$ \\
Frinel_pi & pion fates - inelastic & $\pm 40 \%$ \\
FrAbs_pi & pion fates - absorption & $\pm 30 \%$ \\
FrPiProd_pi & pion fates - pion production & $\pm 20 \%$ \\
AGKYxF1pi & AGKY hadronization model x_F & $\pm 20 \%$ \\
Theta_Delta2Npi & $\Delta$ decay angular distribution & on/off \\
RDecBR1gamma & Res decay branching ratio to gamma & $\pm 50 \%$ \\
\hline \hline
\end{tabular}

Table 32: Final state interaction uncertainties(reweightables) implemented in GENIE. 


\begin{tabular}{|c|c|c|c|c|c|c|c|c|c|}
\hline \hline Source & $\begin{array}{c}2.5 \\
\mathrm{GeV}\end{array}$ & $\begin{array}{c}3.5 \\
\mathrm{GeV}\end{array}$ & $\begin{array}{c}4.5 \\
\mathrm{GeV}\end{array}$ & $\begin{array}{c}6 \\
\mathrm{GeV}\end{array}$ & $\begin{array}{c}8 \\
\mathrm{GeV}\end{array}$ & $\begin{array}{c}10.5 \\
\mathrm{GeV}\end{array}$ & $\begin{array}{c}13.5 \\
\mathrm{GeV}\end{array}$ & $\begin{array}{c}16.5 \\
\mathrm{GeV}\end{array}$ & $\begin{array}{c}20 \\
\mathrm{GeV}\end{array}$ \\
\hline MFP pi & 0.0178 & 0.0139 & 0.0108 & 0.0076 & 0.0027 & 0.0007 & 0.0015 & 0.0008 & 0.0021 \\
MFP N & 0.0024 & 0.0015 & 0.0007 & 0.0005 & 0.0007 & 0.0010 & 0.0009 & 0.0009 & 0.0006 \\
FrAbs pi & 0.0001 & 0.0013 & 0.0005 & 0.0017 & 0.0005 & 0.0013 & 0.0010 & 0.0005 & 0.0027 \\
FrAbs N & 0.0009 & 0.0010 & 0.0009 & 0.0008 & 0.0006 & 0.0007 & 0.0004 & 0.0000 & 0.0008 \\
FrCEx pi & 0.0013 & 0.0003 & 0.0004 & 0.0003 & 0.0000 & 0.0001 & 0.0004 & 0.0003 & 0.0005 \\
FrCEx N & 0.0047 & 0.0039 & 0.0029 & 0.0028 & 0.0014 & 0.0010 & 0.0004 & 0.0003 & 0.0003 \\
FrElas pi & 0.0002 & 0.0002 & 0.0001 & 0.0002 & 0.0001 & 0.0004 & 0.0001 & 0.0001 & 0.0000 \\
FrElas N & 0.0056 & 0.0035 & 0.0031 & 0.0026 & 0.0016 & 0.0020 & 0.0002 & 0.0007 & 0.0007 \\
FrInel pi & 0.0015 & 0.0014 & 0.0007 & 0.0015 & 0.0004 & 0.0015 & 0.0021 & 0.0004 & 0.0040 \\
FrInel N & 0.0013 & 0.0026 & 0.0016 & 0.0016 & 0.0010 & 0.0005 & 0.0007 & 0.0005 & 0.0008 \\
FrPiProd pi & 0.0002 & 0.0003 & 0.0002 & 0.0002 & 0.0002 & 0.0002 & 0.0000 & 0.0002 & 0.0002 \\
FrPiProd N & 0.0001 & 0.0001 & 0.0001 & 0.0002 & 0.0001 & 0.0004 & 0.0002 & 0.0003 & 0.0000 \\
AGKYxF1pi & 0.0018 & 0.0019 & 0.0052 & 0.0068 & 0.0058 & 0.0031 & 0.0013 & 0.0021 & 0.0003 \\
Delta2Npi & 0.0003 & 0.0010 & 0.0005 & 0.0008 & 0.0000 & 0.0002 & 0.0008 & 0.0009 & 0.0004 \\
RDecBR1gamma & 0.0000 & 0.0000 & 0.0000 & 0.0001 & 0.0001 & 0.0001 & 0.0000 & 0.0000 & 0.0001 \\
\hline \hline
\end{tabular}

Table 33: Breakdown of FSI uncertainty for the neutrino cross section. 


\begin{tabular}{|c|c|c|c|c|c|c|c|c|c|}
\hline \hline Source & $\begin{array}{c}2.5 \\
\mathrm{GeV}\end{array}$ & $\begin{array}{c}3.5 \\
\mathrm{GeV}\end{array}$ & $\begin{array}{c}4.5 \\
\mathrm{GeV}\end{array}$ & $\begin{array}{c}6 \\
\mathrm{GeV}\end{array}$ & $\begin{array}{c}8 \\
\mathrm{GeV}\end{array}$ & $\begin{array}{c}10.5 \\
\mathrm{GeV}\end{array}$ & $\begin{array}{c}13.5 \\
\mathrm{GeV}\end{array}$ & $\begin{array}{c}16.5 \\
\mathrm{GeV}\end{array}$ & $\begin{array}{c}20 \\
\mathrm{GeV}\end{array}$ \\
\hline MFP pi & 0.0195 & 0.0162 & 0.0125 & 0.0110 & 0.0066 & 0.0049 & 0.0039 & 0.0018 & 0.0024 \\
MFP N & 0.0064 & 0.0042 & 0.0044 & 0.0037 & 0.0031 & 0.0004 & 0.0013 & 0.0004 & 0.0006 \\
FrAbs pi & 0.0048 & 0.0032 & 0.0021 & 0.0006 & 0.0009 & 0.0023 & 0.0015 & 0.0026 & 0.0000 \\
FrAbs N & 0.0015 & 0.0003 & 0.0001 & 0.0000 & 0.0012 & 0.0002 & 0.0009 & 0.0006 & 0.0021 \\
FrCEx pi & 0.0014 & 0.0016 & 0.0017 & 0.0010 & 0.0004 & 0.0012 & 0.0005 & 0.0005 & 0.0000 \\
FrCEx N & 0.0007 & 0.0008 & 0.0013 & 0.0018 & 0.0008 & 0.0012 & 0.0013 & 0.0016 & 0.0003 \\
FrElas pi & 0.0014 & 0.0013 & 0.0005 & 0.0007 & 0.0000 & 0.0006 & 0.0000 & 0.0002 & 0.0005 \\
FrElas N & 0.0047 & 0.0020 & 0.0017 & 0.0011 & 0.0015 & 0.0005 & 0.0014 & 0.0012 & 0.0056 \\
FrInel pi & 0.0011 & 0.0012 & 0.0014 & 0.0027 & 0.0004 & 0.0002 & 0.0028 & 0.0030 & 0.0009 \\
FrInel N & 0.0030 & 0.0028 & 0.0030 & 0.0031 & 0.0014 & 0.0003 & 0.0006 & 0.0009 & 0.0018 \\
FrPiProd pi & 0.0002 & 0.0002 & 0.0002 & 0.0002 & 0.0000 & 0.0000 & 0.0002 & 0.0004 & 0.0003 \\
FrPiProd N & 0.0003 & 0.0004 & 0.0003 & 0.0002 & 0.0003 & 0.0003 & 0.0000 & 0.0005 & 0.0003 \\
AGKYxF1pi & 0.0042 & 0.0005 & 0.0035 & 0.0054 & 0.0069 & 0.0049 & 0.0059 & 0.0019 & 0.0010 \\
Delta2Npi & 0.0001 & 0.0000 & 0.0000 & 0.0001 & 0.0001 & 0.0000 & 0.0000 & 0.0001 & 0.0000 \\
RDecBR1gamma & 0.0002 & 0.0001 & 0.0000 & 0.0001 & 0.0001 & 0.0000 & 0.0002 & 0.0001 & 0.0000 \\
\hline \hline
\end{tabular}

Table 34: Breakdown of FSI uncertainty for the antineutrino cross section. 


\begin{tabular}{|c|c|c|c|c|c|c|c|c|c|}
\hline \hline Source & 2.5 & 3.5 & 4.5 & 6 & 8 & 10.5 & 13.5 & 16.5 & 20 \\
& $\mathrm{GeV}$ & $\mathrm{GeV}$ & $\mathrm{GeV}$ & $\mathrm{GeV}$ & $\mathrm{GeV}$ & $\mathrm{GeV}$ & $\mathrm{GeV}$ & $\mathrm{GeV}$ & $\mathrm{GeV}$ \\
\hline MFP pi & 0.0017 & 0.0023 & 0.0016 & 0.0034 & 0.0039 & 0.0042 & 0.0024 & 0.0026 & 0.0045 \\
MFP N & 0.0088 & 0.0026 & 0.0037 & 0.0032 & 0.0024 & 0.0006 & 0.0022 & 0.0005 & 0.0013 \\
FrAbs pi & 0.0049 & 0.0045 & 0.0026 & 0.0022 & 0.0014 & 0.0010 & 0.0025 & 0.0030 & 0.0027 \\
FrAbs N & 0.0024 & 0.0006 & 0.0008 & 0.0008 & 0.0018 & 0.0009 & 0.0005 & 0.0007 & 0.0013 \\
FrCEx pi & 0.0000 & 0.0013 & 0.0020 & 0.0013 & 0.0004 & 0.0011 & 0.0009 & 0.0003 & 0.0005 \\
FrCEx N & 0.0040 & 0.0030 & 0.0016 & 0.0010 & 0.0006 & 0.0002 & 0.0016 & 0.0018 & 0.0000 \\
FrElas pi & 0.0017 & 0.0012 & 0.0004 & 0.0005 & 0.0001 & 0.0002 & 0.0000 & 0.0003 & 0.0005 \\
FrElas N & 0.0103 & 0.0055 & 0.0048 & 0.0038 & 0.0031 & 0.0015 & 0.0013 & 0.0005 & 0.0050 \\
FrInel pi & 0.0026 & 0.0001 & 0.0021 & 0.0012 & 0.0008 & 0.0017 & 0.0049 & 0.0026 & 0.0031 \\
FrInel N & 0.0016 & 0.0003 & 0.0013 & 0.0014 & 0.0005 & 0.0002 & 0.0001 & 0.0014 & 0.0025 \\
FrPiProd pi & 0.0003 & 0.0005 & 0.0004 & 0.0004 & 0.0002 & 0.0002 & 0.0003 & 0.0006 & 0.0005 \\
FrPiProd N & 0.0003 & 0.0003 & 0.0002 & 0.0000 & 0.0004 & 0.0007 & 0.0003 & 0.0008 & 0.0004 \\
AGKYxF1pi & 0.0024 & 0.0024 & 0.0017 & 0.0014 & 0.0011 & 0.0018 & 0.0046 & 0.0040 & 0.0007 \\
Delta2Npi & 0.0002 & 0.0011 & 0.0005 & 0.0009 & 0.0001 & 0.0002 & 0.0008 & 0.0008 & 0.0004 \\
RDecBR1gamma & 0.0001 & 0.0000 & 0.0000 & 0.0001 & 0.0002 & 0.0001 & 0.0002 & 0.0001 & 0.0001 \\
\hline \hline
\end{tabular}

Table 35: Breakdown of FSI uncertainty for the ratio, $r$. 


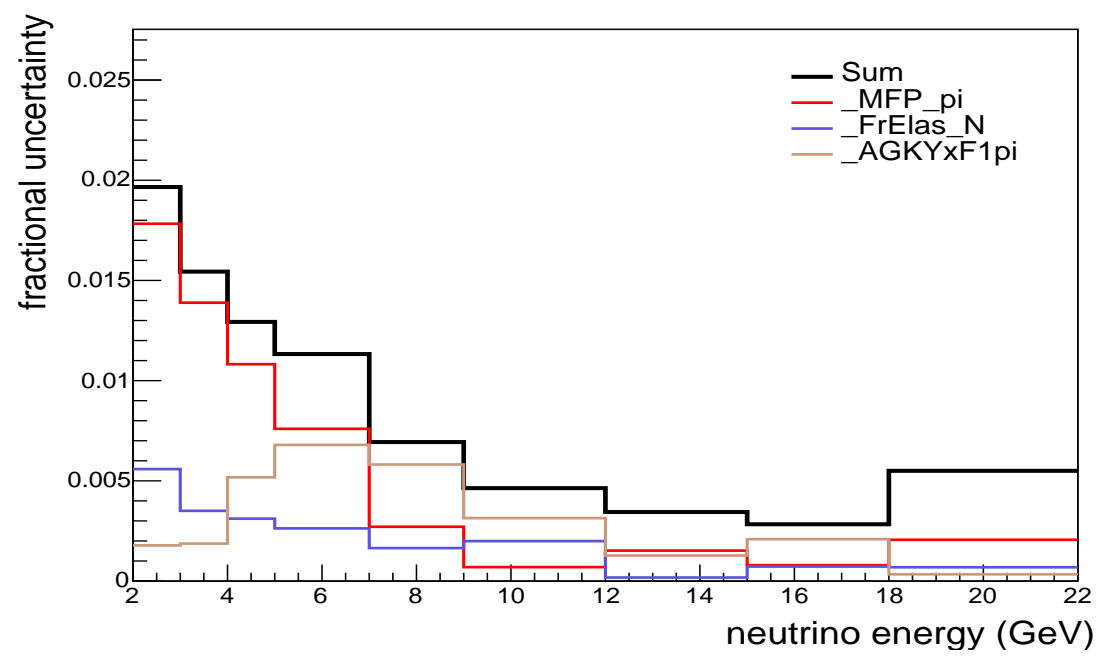

Figure 66: Size of GENIE-based FSI model uncertainty for neutrino cross section.

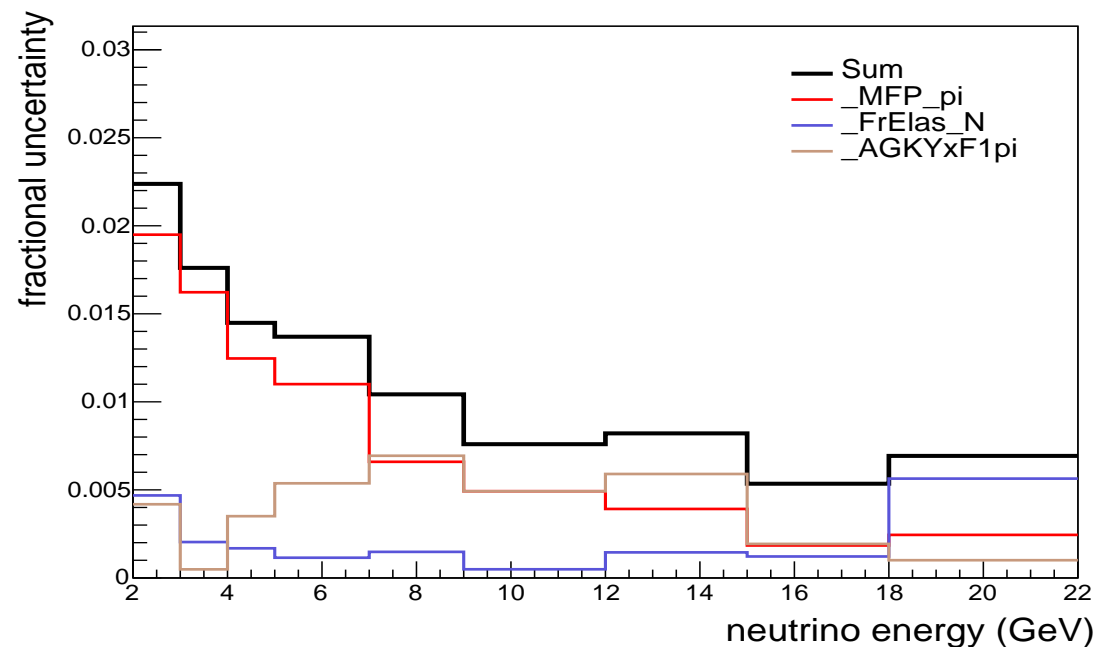

Figure 67: Size of GENIE-based FSI model uncertainty for antineutrino cross section. 


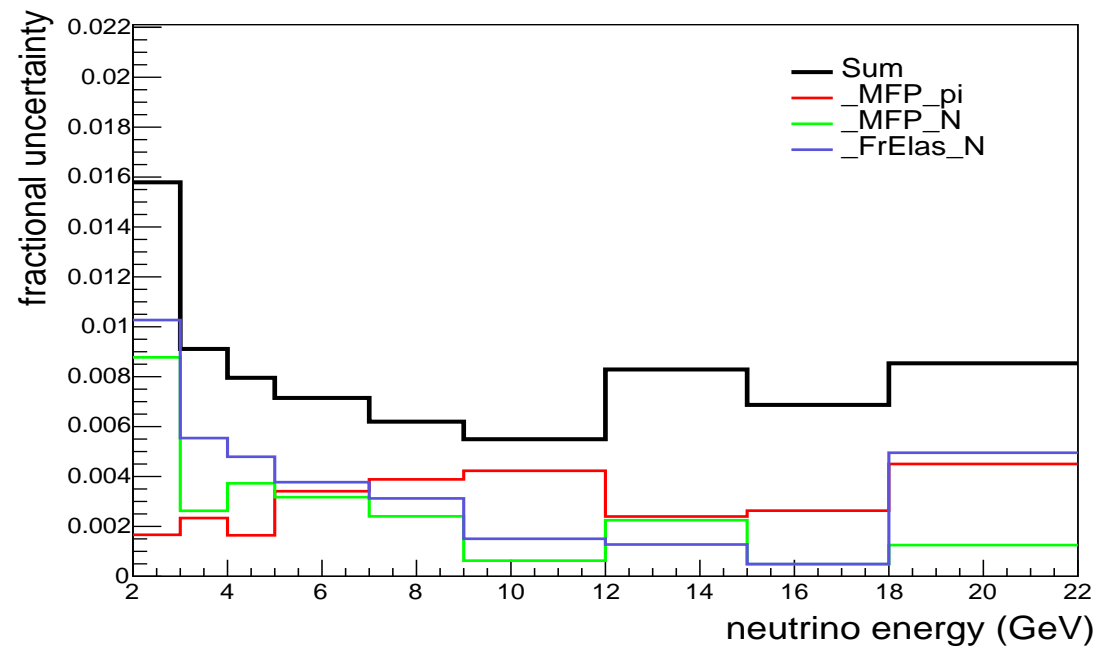

Figure 68: Size of GENIE-based FSI model uncertainty for cross section ratio $r$.

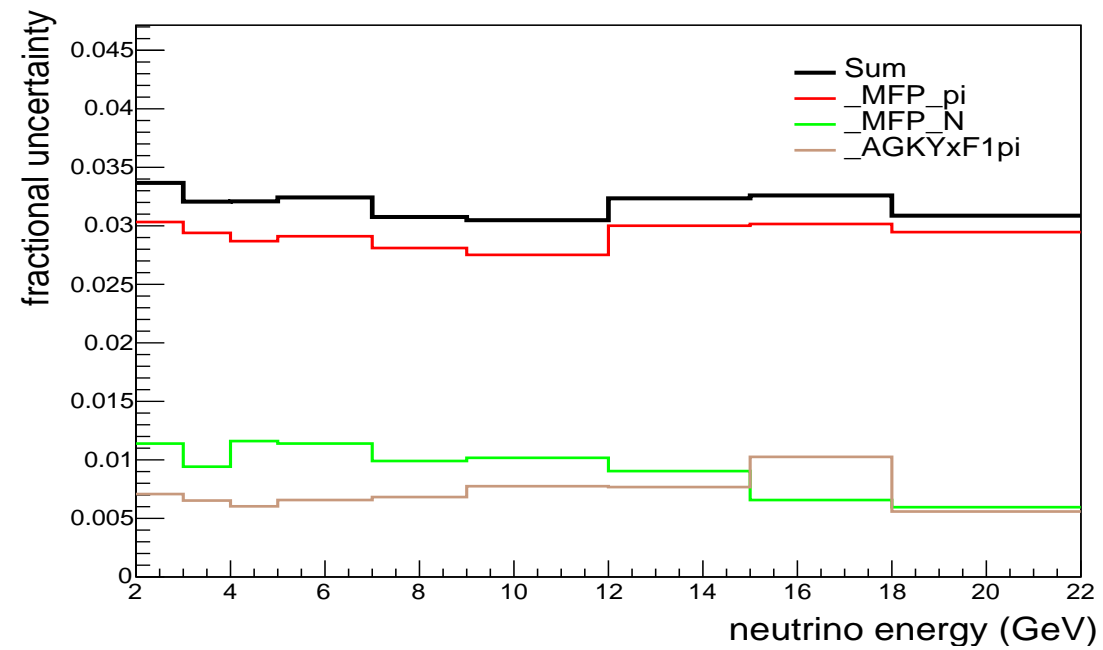

Figure 69: FSI uncertainty of extracted FHC neutrino flux. It shows the sum of contributions from each component and three components with the largest contribution. 


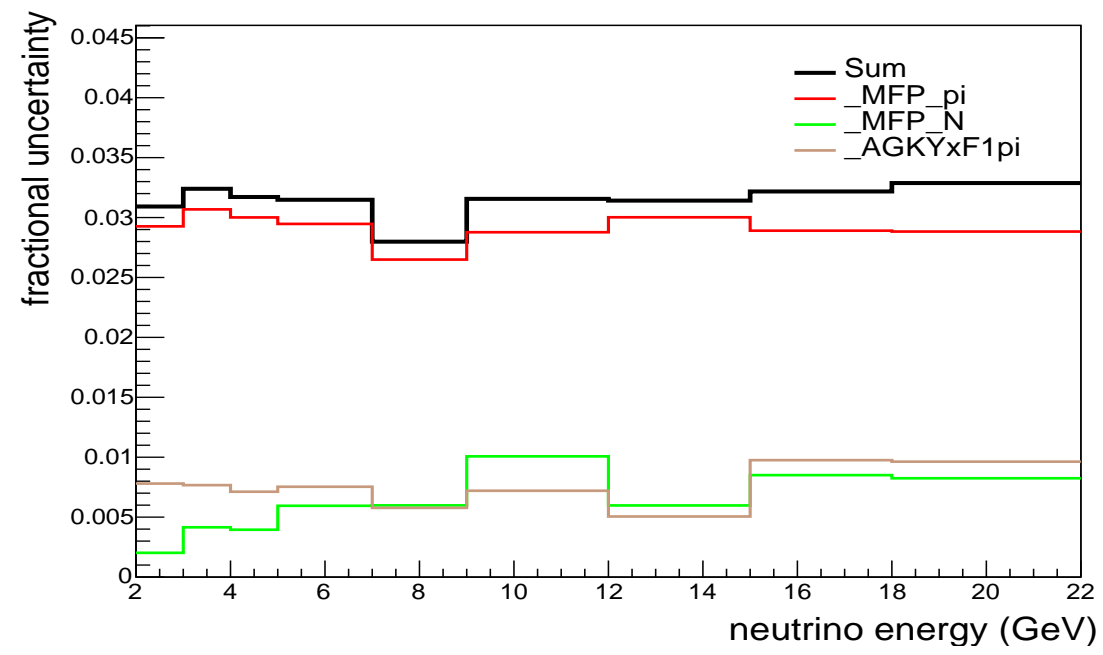

Figure 70: FSI uncertainty of extracted RHC antineutrino flux. It shows the sum of contributions from each component and three components with the largest contribution.

\begin{tabular}{cc}
\hline \hline EFNUCR & Increase/decrease to nuclear size for low energy hadrons $( \pm 0.6 \mathrm{fm})$. \\
FZONE & Change formation time by $50 \%$ \\
Hadronization_Alt1 & Change AGKY model to do a simple phase space decay of hadrons.
\end{tabular}

Table 36: Final state interaction uncertainties (non-reweightables) not implemented in GENIE . 


\subsection{CROSS SECTION MODEL UNCERTAINTIES}

The generator-level cross section model has an effect on all extracted model-based corrections needed for this measurement. Tab. 37 lists cross section model uncertainties and their values

considered. All are implemented with reweighting in GENIE MC (GENIE reweightable), except for the last two 2p2h and RPA model uncertainties, which are evaluated separately. Uncertainties of GENIE reweightable uncertainties, $2 \mathrm{p} 2 \mathrm{~h}$ and RPA model uncertainties are added in quadrature in error summary plots as cross section model uncertainty.

\subsubsection{GENIE reweightable uncertainties}

For each GENIE source in the table, we compute shifted values for kinematic correction $A_{C C}^{K I N}$, and for low- $\nu$ correction $S^{\nu, \bar{\nu}}$ for the model. The effect of cross section model uncertainties is negligible for the isoscalar correction $I^{\text {iso }}$ and $G_{\text {corr }}$.

The fractional difference from the nominal cross section is taken as the error. Each source is evaluated independently and added in quadrature to obtain a combined error band. Fig. 71, Fig. 72 and Fig. 73 show the uncertainties in neutrino, antineutrino cross sections and the ratio, $r$, respectively, from each source. This uncertainty is dominated by uncertainty of parameter $M_{A}$ and $M_{V}$ in Rein-Sehgal model, since for the first four bins, we use $\nu<0.3 \mathrm{GeV}$ and $\nu<0.5 \mathrm{GeV}$ cut which is at the range where resonance piece has a dominated contribution.

Fig. 74 shows the breakdown of GENIE cross section model uncertainty for extracted FHC neutrino flux, similarly as neutrino cross section, it is also dominated by $M_{A}$ and $M_{V}$ of ReinSeghal model. Fig. 75 shows the breakdown of GENIE cross section model uncertainty for extracted RHC antineutrino flux. 


\begin{tabular}{ccc}
\hline \hline GENIE Knob name & Description & $1 \sigma$ \\
\hline MaRES & adjust $M_{A}$ in Rein-Sehgal cross section & $\pm 20 \%$ \\
MvRES & adjust $M_{v}$ in Rein-Sehgal cross section & $\pm 10 \%$ \\
Rvp1pi & 1 pi production from $\nu p$ non-resonant interactions & $\pm 50 \%$ \\
Rvn1pi & 1 pi production from $\nu n$ non-resonant interactions & $\pm 15 \%$ \\
Rvp2pi & 2 pi production from $\nu p$ non-resonant interactions & $\pm 50 \%$ \\
Rvn2pi & 2 pi production from $\nu n$ non-resonant interactions & $\pm 50 \%$ \\
VeCFFCCQEshape & Changes from BBBA to dipole & on or off \\
AhtBY & Bodek-Yang parameter $A_{H T}$ & $\pm 25 \%$ \\
BhtBY & Bodek-Yang parameter $B_{H T}$ & $\pm 25 \%$ \\
CV1uBY & Bodek-Yang parameter $C_{V 1 u}$ & $\pm 30 \%$ \\
CV2uBY & Bodek-Yang parameter $C_{V 2 u}$ & $\pm 40 \%$ \\
\hline 2p2h & reweighting 2p2h model & on or off \\
RPA & reweighting RPA model & on or off \\
\hline
\end{tabular}

Table 37: Cross section model uncertainties considered [1]. 


\begin{tabular}{|c|c|c|c|c|c|c|c|c|c|}
\hline Source & $\begin{array}{c}2.5 \\
\mathrm{GeV}\end{array}$ & $\begin{array}{c}3.5 \\
\mathrm{GeV}\end{array}$ & $\begin{array}{c}4.5 \\
\mathrm{GeV}\end{array}$ & $\begin{array}{c}6 \\
\mathrm{GeV}\end{array}$ & $\begin{array}{c}8 \\
\mathrm{GeV}\end{array}$ & $\begin{array}{l}10.5 \\
\mathrm{GeV}\end{array}$ & $\begin{array}{l}13.5 \\
\mathrm{GeV}\end{array}$ & $\begin{array}{l}16.5 \\
\mathrm{GeV}\end{array}$ & $\begin{array}{c}20 \\
\mathrm{GeV}\end{array}$ \\
\hline MaRES & 0.0441 & 0.0016 & 0.0112 & 0.0146 & 0.0102 & 0.0060 & 0.0027 & 0.0020 & 0.0021 \\
\hline Rvp2pi & 234 & 0.0061 & 0.0025 & 0.004 & 0.0029 & 0.0016 & 0.0005 & 0.0001 & 0.0009 \\
\hline Rvp1pi & 0.0078 & 0.0033 & 0.0007 & 0.0017 & 0.0013 & 0.0008 & 0.0002 & 0.0001 & 0.0003 \\
\hline Rvn2pi & 0.0265 & 0.0162 & 0.0050 & 0.0008 & 0.00 & 0.0015 & 0.0006 & 0.0002 & 0.0007 \\
\hline Rvn1pi & 0.0396 & 0.0184 & 0.0014 & 0.0055 & 0.0051 & 0.0030 & 0.0007 & 0.0001 & 0.0013 \\
\hline MvRES & 0.0290 & 0.0023 & 0.0055 & 0.0073 & 0.0050 & 0.0029 & 0.0012 & 0.0008 & 0.0011 \\
\hline CCQEPauli & 0.0190 & 0.0065 & 0.0036 & 0.0020 & 0.0009 & 0.0004 & 0.0001 & 0.0000 & 0.00 \\
\hline VecFFCCQ & 0.0051 & 0.0007 & 0.0011 & 0.0013 & 0.0006 & 0.0004 & 0.0002 & 0.0001 & 0.0002 \\
\hline AhtBY & 0.0021 & 0.0063 & 0.0082 & 0.0065 & 0.0036 & 0.0014 & 0.0002 & 0.0001 & 0.0004 \\
\hline BhtBY & 0.0045 & 0.0107 & 0.0132 & 0.0103 & 0.0055 & 0.0025 & 0.0011 & 0.0008 & 0.0009 \\
\hline CV1uBY & 0.0029 & 0.0057 & 0.0067 & 0.0043 & 0.0018 & 0.0006 & 0.0001 & 0.0001 & 0.0002 \\
\hline CV2uBY & 0.0028 & 0.0058 & 0.0063 & 0.0039 & 0.0017 & 0.0005 & 0.0000 & 0.0000 & 0.0000 \\
\hline
\end{tabular}

Table 38: Breakdown of cross section model uncertainty for neutrino cross section. 


\begin{tabular}{|c|c|c|c|c|c|c|c|c|c|}
\hline \hline Source & 2.5 & 3.5 & 4.5 & 6 & 8 & 10.5 & 13.5 & 16.5 & $\begin{array}{c}20 \\
\mathrm{GeV}\end{array}$ \\
& $\mathrm{GeV}$ & $\mathrm{GeV}$ & $\mathrm{GeV}$ & $\mathrm{GeV}$ & $\mathrm{GeV}$ & $\mathrm{GeV}$ & $\mathrm{GeV}$ & $\mathrm{GeV}$ \\
\hline MaRES & 0.0419 & 0.0131 & 0.0050 & 0.0000 & 0.0020 & 0.0016 & 0.0022 & 0.0063 & 0.0063 \\
Rvp2pi & 0.0131 & 0.0044 & 0.0012 & 0.0002 & 0.0009 & 0.0009 & 0.0011 & 0.0013 & 0.0013 \\
Rvp1pi & 0.0047 & 0.0024 & 0.0006 & 0.0003 & 0.0003 & 0.0003 & 0.0005 & 0.0005 & 0.0006 \\
Rvn2pi & 0.0165 & 0.0091 & 0.0041 & 0.0019 & 0.0010 & 0.0008 & 0.0012 & 0.0018 & 0.0017 \\
Rvn1pi & 0.0239 & 0.0115 & 0.0043 & 0.0016 & 0.0021 & 0.0019 & 0.0029 & 0.0025 & 0.0023 \\
MvRES & 0.0147 & 0.0055 & 0.0023 & 0.0009 & 0.0038 & 0.0031 & 0.0029 & 0.0049 & 0.0046 \\
CCQEPauli & 0.0152 & 0.0037 & 0.0017 & 0.0006 & 0.0001 & 0.0001 & 0.0002 & 0.0003 & 0.0003 \\
VecFFCCQE & 0.0024 & 0.0035 & 0.0033 & 0.0028 & 0.0022 & 0.0016 & 0.0012 & 0.0008 & 0.0007 \\
AhtBY & 0.0011 & 0.0006 & 0.0006 & 0.0002 & 0.0011 & 0.0007 & 0.0003 & 0.0008 & 0.0005 \\
BhtBY & 0.0004 & 0.0012 & 0.0004 & 0.0005 & 0.0002 & 0.0003 & 0.0000 & 0.0018 & 0.0014 \\
CV1uBY & 0.0007 & 0.0016 & 0.0019 & 0.0013 & 0.0006 & 0.0005 & 0.0004 & 0.0001 & 0.0001 \\
CV2uBY & 0.0007 & 0.0011 & 0.0016 & 0.0008 & 0.0003 & 0.0001 & 0.0000 & 0.0005 & 0.0005 \\
\hline \hline
\end{tabular}

Table 39: Breakdown of cross section model uncertainty for antineutrino cross section. 


\begin{tabular}{|c|c|c|c|c|c|c|c|c|c|}
\hline \hline Source & 2.5 & 3.5 & 4.5 & 6 & 8 & 10.5 & 13.5 & 16.5 & $\begin{array}{c}20 \\
\mathrm{GeV}\end{array}$ \\
& $\mathrm{GeV}$ & $\mathrm{GeV}$ & $\mathrm{GeV}$ & $\mathrm{GeV}$ & $\mathrm{GeV}$ & $\mathrm{GeV}$ & $\mathrm{GeV}$ & $\mathrm{GeV}$ \\
\hline MaRES & 0.0023 & 0.0115 & 0.0162 & 0.0146 & 0.0122 & 0.0076 & 0.0048 & 0.0043 & 0.0042 \\
Rvp2pi & 0.0104 & 0.0017 & 0.0037 & 0.0045 & 0.0038 & 0.0025 & 0.0016 & 0.0013 & 0.0004 \\
Rvp1pi & 0.0030 & 0.0009 & 0.0014 & 0.0020 & 0.0016 & 0.0011 & 0.0007 & 0.0005 & 0.0003 \\
Rvn2pi & 0.0100 & 0.0071 & 0.0009 & 0.0027 & 0.0032 & 0.0023 & 0.0017 & 0.0016 & 0.0010 \\
Rvn1pi & 0.0157 & 0.0068 & 0.0029 & 0.0071 & 0.0073 & 0.0049 & 0.0035 & 0.0026 & 0.0011 \\
MvRES & 0.0143 & 0.0032 & 0.0079 & 0.0082 & 0.0088 & 0.0060 & 0.0041 & 0.0041 & 0.0034 \\
CCQEPauli & 0.0038 & 0.0028 & 0.0020 & 0.0014 & 0.0008 & 0.0005 & 0.0003 & 0.0002 & 0.0001 \\
VecFFCCQE & 0.0075 & 0.0041 & 0.0022 & 0.0014 & 0.0016 & 0.0012 & 0.0010 & 0.0010 & 0.000 \\
AhtBY & 0.0032 & 0.0069 & 0.0088 & 0.0068 & 0.0025 & 0.0006 & 0.0002 & 0.0007 & 0.0010 \\
BhtBY & 0.0049 & 0.0119 & 0.0135 & 0.0098 & 0.0057 & 0.0023 & 0.0010 & 0.0011 & 0.0006 \\
CV1uBY & 0.0022 & 0.0041 & 0.0048 & 0.0030 & 0.0012 & 0.0002 & 0.0003 & 0.0000 & 0.0001 \\
CV2uBY & 0.0021 & 0.0048 & 0.0048 & 0.0031 & 0.0014 & 0.0004 & 0.0000 & 0.0006 & 0.0005 \\
\hline \hline
\end{tabular}

Table 40: Breakdown of cross section model uncertainty for the ratio, $r$. 


\begin{tabular}{|c|c|c|c|c|c|c|c|c|c|}
\hline \hline knob & 2.5 & 3.5 & 4.5 & 6 & 8 & 10.5 & 13.5 & 16.5 & $\begin{array}{c}20 \\
\mathrm{GeV}\end{array}$ \\
$\mathrm{GeV}$ & $\mathrm{GeV}$ & $\mathrm{GeV}$ & $\mathrm{GeV}$ & $\mathrm{GeV}$ & $\mathrm{GeV}$ & $\mathrm{GeV}$ & $\mathrm{GeV}$ \\
\hline MaRES & 0.0029 & 0.0049 & 0.0045 & 0.0040 & 0.0047 & 0.0043 & 0.0040 & 0.0066 & 0.0052 \\
Rvp2pi & 0.0015 & 0.0016 & 0.0016 & 0.0016 & 0.0020 & 0.0019 & 0.0018 & 0.0015 & 0.0020 \\
Rvp1pi & 0.0009 & 0.0011 & 0.0009 & 0.0009 & 0.0009 & 0.0008 & 0.0008 & 0.0007 & 0.0007 \\
Rvn2pi & 0.0019 & 0.0019 & 0.0019 & 0.0019 & 0.0020 & 0.0020 & 0.0019 & 0.0019 & 0.0019 \\
Rvn1pi & 0.0038 & 0.0050 & 0.0042 & 0.0039 & 0.0046 & 0.0042 & 0.0042 & 0.0033 & 0.0036 \\
MvRES & 0.0018 & 0.0022 & 0.0021 & 0.0020 & 0.0024 & 0.0021 & 0.0020 & 0.0030 & 0.0027 \\
CCQEPauli & 0.0004 & 0.0004 & 0.0004 & 0.0004 & 0.0004 & 0.0004 & 0.0004 & 0.0004 & 0.0004 \\
VecFFCCQE & 0.0057 & 0.0026 & 0.0020 & 0.0014 & 0.0006 & 0.0003 & 0.0002 & 0.0001 & 0.0002 \\
AhtBY & 0.0010 & 0.0010 & 0.0008 & 0.0009 & 0.0009 & 0.0010 & 0.0009 & 0.0004 & 0.0006 \\
BhtBY & 0.0004 & 0.0004 & 0.0003 & 0.0003 & 0.0004 & 0.0004 & 0.0002 & 0.0013 & 0.0010 \\
CV1uBY & 0.0005 & 0.0003 & 0.0005 & 0.0005 & 0.0005 & 0.0006 & 0.0006 & 0.0005 & 0.0005 \\
CV2uBY & 0.0006 & 0.0009 & 0.0007 & 0.0006 & 0.0006 & 0.0005 & 0.0005 & 0.0005 & 0.0006 \\
\hline \hline
\end{tabular}

Table 41: Breakdown of cross section model uncertainty for neutrino flux. 


\begin{tabular}{|c|c|c|c|c|c|c|c|c|c|}
\hline \hline knob & $\begin{array}{c}2.5 \\
\mathrm{GeV}\end{array}$ & $\begin{array}{c}3.5 \\
\mathrm{GeV}\end{array}$ & $\begin{array}{c}4.5 \\
\mathrm{GeV}\end{array}$ & $\begin{array}{c}6 \\
\mathrm{GeV}\end{array}$ & $\begin{array}{c}8 \\
\mathrm{GeV}\end{array}$ & $\begin{array}{c}10.5 \\
\mathrm{GeV}\end{array}$ & $\begin{array}{c}13.5 \\
\mathrm{GeV}\end{array}$ & $\begin{array}{c}16.5 \\
\mathrm{GeV}\end{array}$ & $\begin{array}{c}20 \\
\mathrm{GeV}\end{array}$ \\
\hline MaRES & 0.0029 & 0.0029 & 0.0029 & 0.0028 & 0.0052 & 0.0047 & 0.0041 & 0.0076 & 0.0073 \\
Rvp2pi & 0.0015 & 0.0015 & 0.0016 & 0.0015 & 0.0020 & 0.0019 & 0.0017 & 0.0017 & 0.0016 \\
Rvp1pi & 0.0009 & 0.0007 & 0.0008 & 0.0008 & 0.0008 & 0.0008 & 0.0008 & 0.0007 & 0.0007 \\
Rvn2pi & 0.0019 & 0.0019 & 0.0019 & 0.0019 & 0.0021 & 0.0020 & 0.0020 & 0.0024 & 0.0022 \\
Rvn1pi & 0.0038 & 0.0036 & 0.0034 & 0.0034 & 0.0047 & 0.0041 & 0.0043 & 0.0036 & 0.0032 \\
MvRES & 0.0017 & 0.0035 & 0.0031 & 0.0027 & 0.0052 & 0.0044 & 0.0037 & 0.0054 & 0.0050 \\
CCQEPauli & 0.0004 & 0.0004 & 0.0004 & 0.0004 & 0.0004 & 0.0004 & 0.0004 & 0.0004 & 0.0004 \\
VecFF & 0.0064 & 0.0051 & 0.0038 & 0.0028 & 0.0022 & 0.0016 & 0.0012 & 0.0008 & 0.0007 \\
AhtBY & 0.0010 & 0.0004 & 0.0007 & 0.0007 & 0.0004 & 0.0001 & 0.0000 & 0.0007 & 0.0004 \\
BhtBY & 0.0003 & 0.0010 & 0.0006 & 0.0006 & 0.0018 & 0.0014 & 0.0012 & 0.0025 & 0.0022 \\
CV1uBY & 0.0005 & 0.0007 & 0.0006 & 0.0007 & 0.0006 & 0.0007 & 0.0007 & 0.0002 & 0.0002 \\
CV2uBY & 0.0005 & 0.0003 & 0.0004 & 0.0003 & 0.0004 & 0.0003 & 0.0003 & 0.0008 & 0.0008 \\
\hline \hline
\end{tabular}

Table 42: Breakdown of cross section model uncertainty for antineutrino flux. 


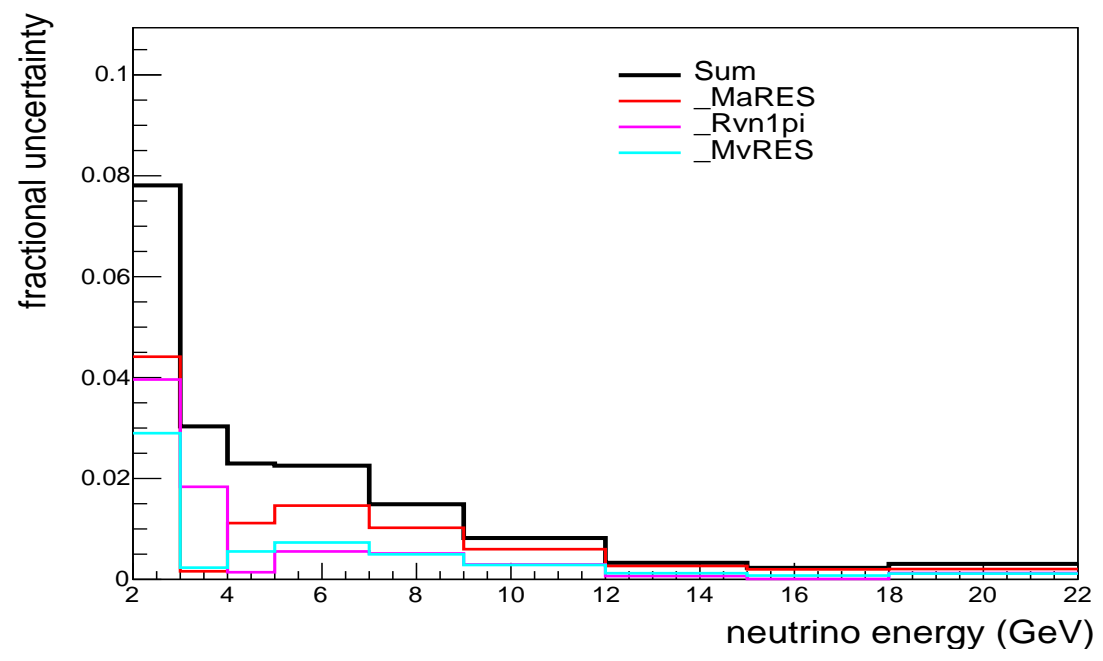

Figure 71: Cross section model uncertainty for the neutrino cross section.

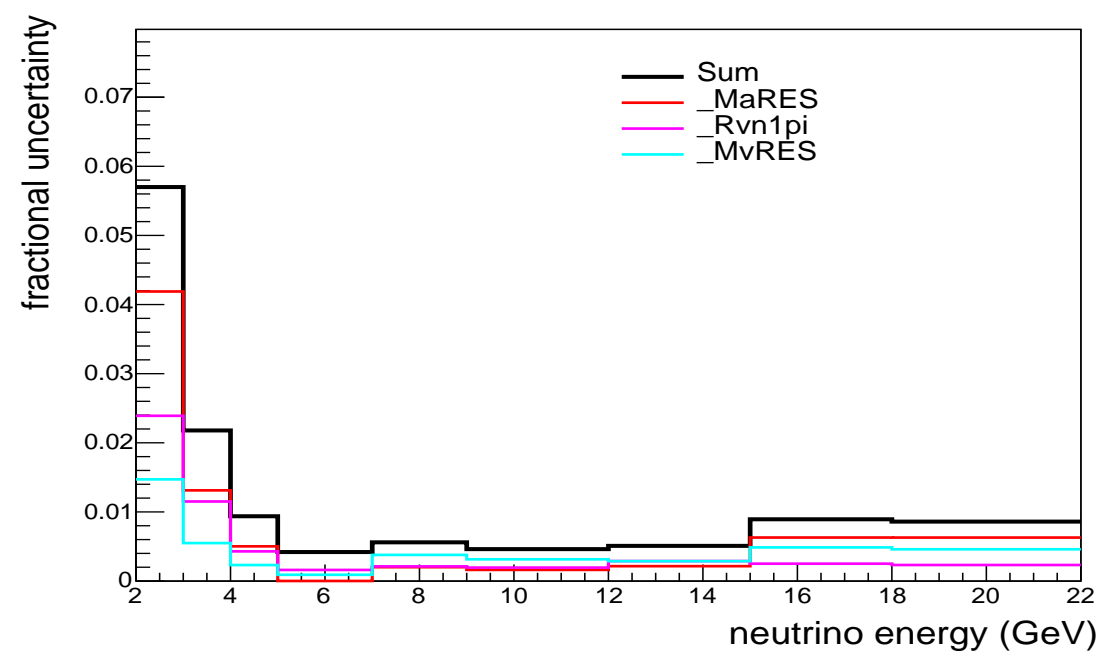

Figure 72: Cross section model uncertainty for the antineutrino cross section. 


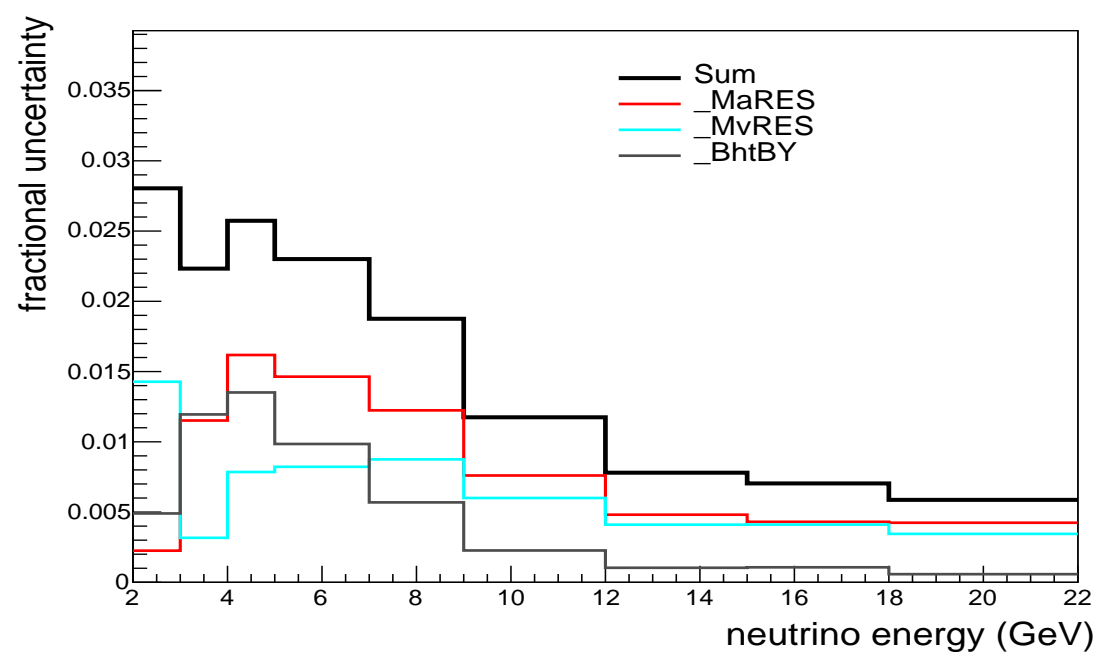

Figure 73: Cross section model uncertainty for the ratio, $r$.

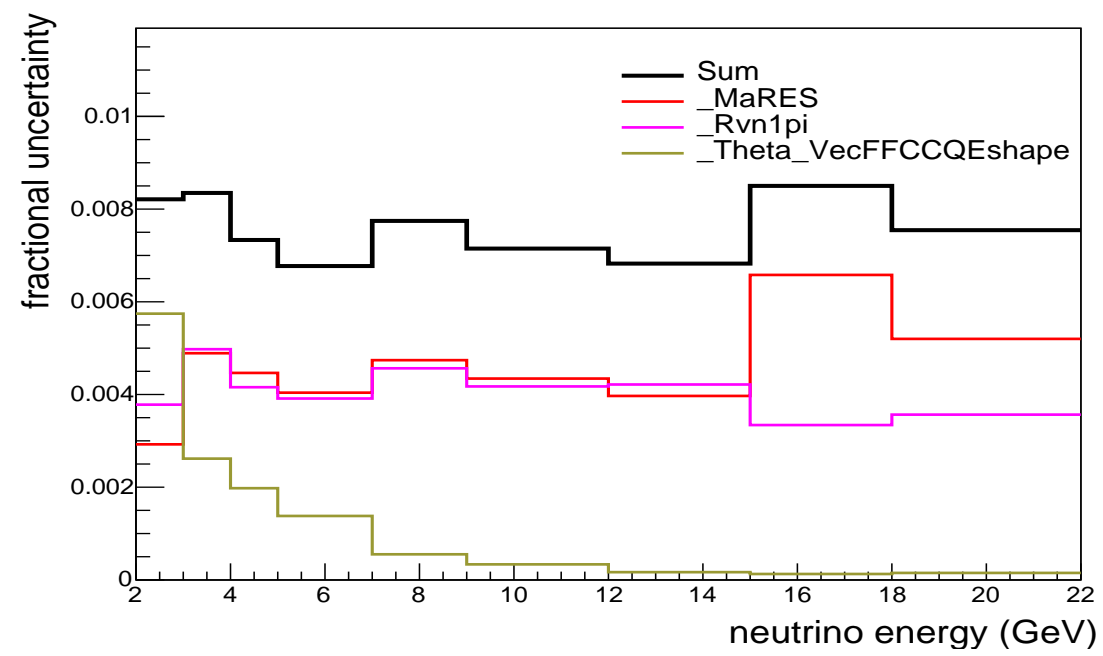

Figure 74: Cross section model uncertainty of extracted FHC neutrino flux. It shows the sum of contributions from each component and three components with the largest contribution. 


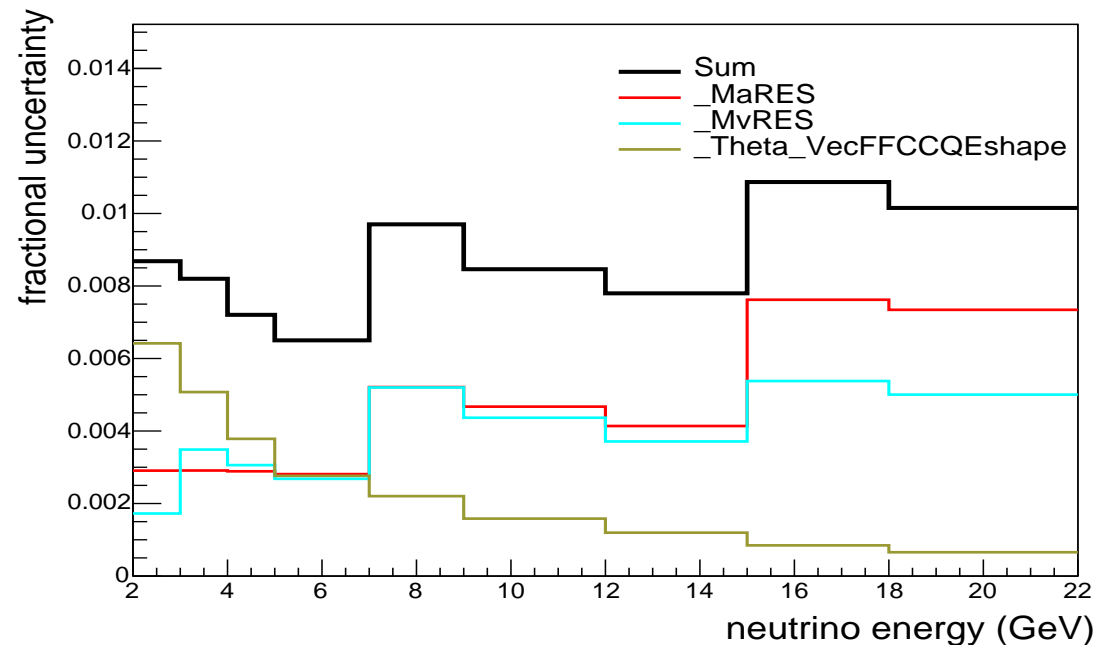

Figure 75: Cross section model uncertainty of extracted RHC antineutrino flux. It shows the sum of contributions from each component and three components with the largest contribution.

\subsubsection{RPA}

The systematic of RPA is evaluated by turning on and off the RPA weight shown in Fig 24, half the change in each measured quantity is assigned as the uncertainty. The size of the RPA uncertainty is shown together with that from $2 \mathrm{p} 2 \mathrm{~h}$ in the next subsection.

\subsubsection{2p2h Model Uncertainty}

Fig. 76 (taken from [13]) shows the double-differential cross section in six regions of q3 is compared to the GENIE 2.8.4 model with with RPA suppression and then combined with a QE-like 2p2h component (solid). This model is similar to the GENIE-Hybrid model, but with a slightly different ${ }^{2}$ reweighting of the one-pion non-resonance component. The difference between data and model (the solid lines) suggests we introduce a $2 \mathrm{p} 2 \mathrm{~h}$ model uncertainty to account for this discrepancy.

\footnotetext{
${ }^{2}$ We use $57 \%$, while Ref.[13] uses $50 \%$.
} 


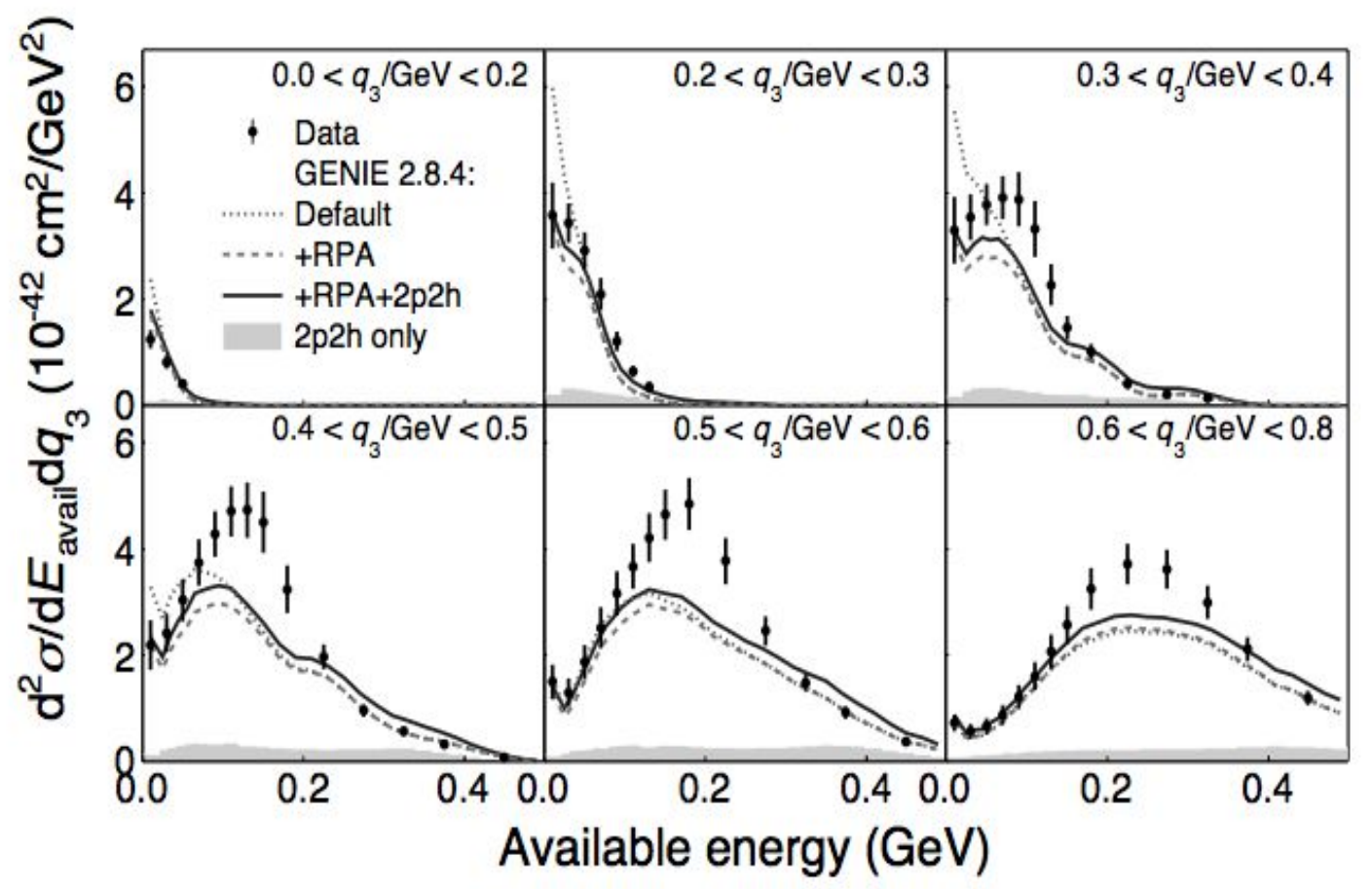

Figure 76: The double-differential cross section in six regions of $\mathrm{q} 3$ is compared to the GENIE 2.8.4 model with RPA suppression (long-dashed), and then combined with a QE- like 2p2h component (solid). (Taken from [13])

A two-step data-driven fit is applied to MC simulation to obtain the $2 \mathrm{p} 2 \mathrm{~h}$ uncertainty. First, a 2-dimensional ( $\left.q 3, E_{\text {avail }}\right)$-dependent reweighting function [14] is defined as shown in Fig. 77. It is applied to the $2 \mathrm{p} 2 \mathrm{~h}$ events with $q 3<0.8 \mathrm{GeV}$. This improves the modeling of available energy in six regions of $q 3$ as shown in Fig. 78. The fit, though evaluated from the neutrino sample, is applied to both neutrino and antineutrino MC simulation samples. After applying the 2-dimensional reweighting, a discrepancy between data and model remains, as shown in Fig. 79. Another fit to the MC simulation is evaluated to minimize remaining differences. This fit treats the initial state of $2 \mathrm{p} 2 \mathrm{~h}$ events separately. We define the hadron energy distribution from MC simulation for neutrino as

$$
T^{\nu}=N^{\nu}+M_{n n}^{\nu} * \alpha+M_{n p}^{\nu} * \beta,
$$




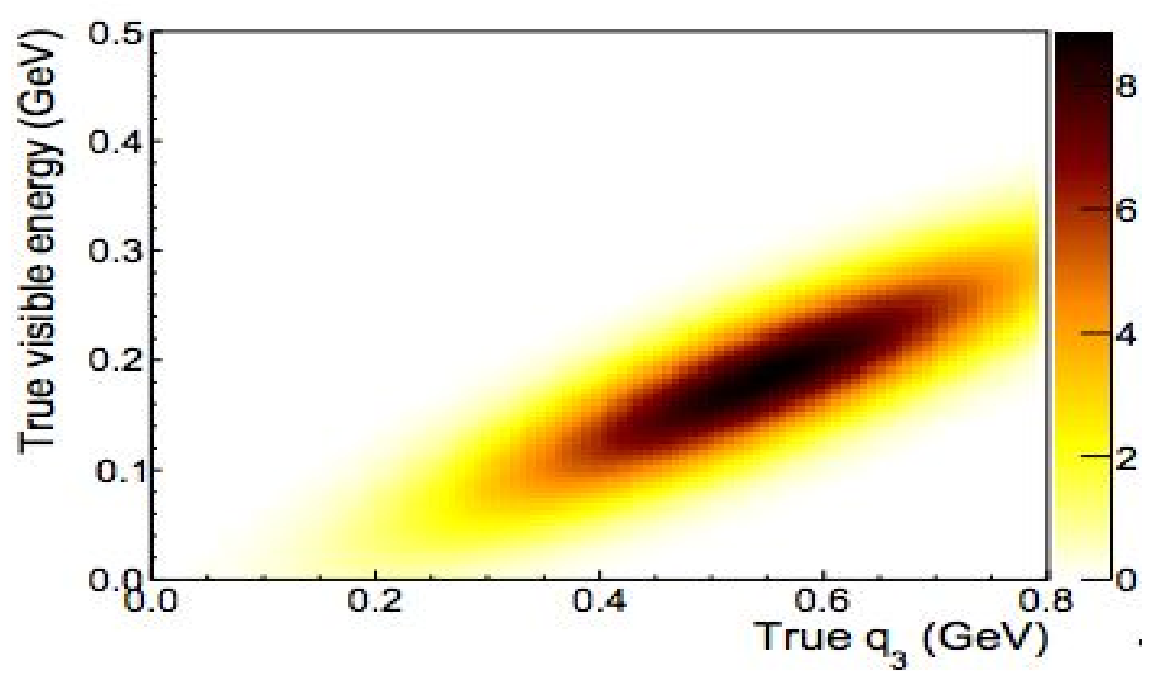

Figure 77: 2p2h reweighting function accounting for the data MC discrepancy shown in Fig. 76. (Taken from [14])

and for antineutrino as

$$
T^{\bar{\nu}}=N^{\bar{\nu}}+M_{p p}^{\bar{\nu}} * \gamma+M_{n p}^{\bar{\nu}} * \delta,
$$

$T^{\nu(\bar{\nu})}$ stands for all MC simulation events, $N^{\nu(\bar{\nu})}$ stands for MC simulation events which are not identified as $2 \mathrm{p} 2 \mathrm{~h}$ events, $M_{p p}^{\nu}$ is the distribution for $2 \mathrm{p} 2 \mathrm{~h}$ events with "pp" in the initial state, $M_{n p}^{\nu(\bar{\nu})}$ is for $2 \mathrm{p} 2 \mathrm{~h}$ events with"np" in the initial state, and $M_{p p}^{\nu}$ is for $2 \mathrm{p} 2 \mathrm{~h}$ events with "pp" in the initial state. Without reweighting we have $\alpha=\beta=\gamma=\delta=1$. The best fit values are $\alpha=0, \beta=1.11$, $\gamma=1.80$ and $\delta=0.78$ and are used as the reweighting constants. These weights are applied only to events with $q 3<0.8 \mathrm{GeV}$. The total effect of this 2-step data-driven fit is shown in Fig. 80. The fit improves the agreement between data and MC, especially in the energy region $E_{h a d}<0.3 \mathrm{GeV}$. The fractional change of measured quantities using this reweighted model from the nominal model is assigned as the 2p2h model uncertainty. Fig. 81, Fig. 82 and Fig. 83 show the size of 2p2h (and RPA) uncertainty for neutrino, antineutrino cross sections and $r$, respectively. The uncertainty for cross sections is less than $2 \%$, since the major effect in $A^{d e t}$ and $A^{\phi}$ terms are flat as a function of neutrino energy, and the resulting overall shift is taken out by the normalization procedure. 


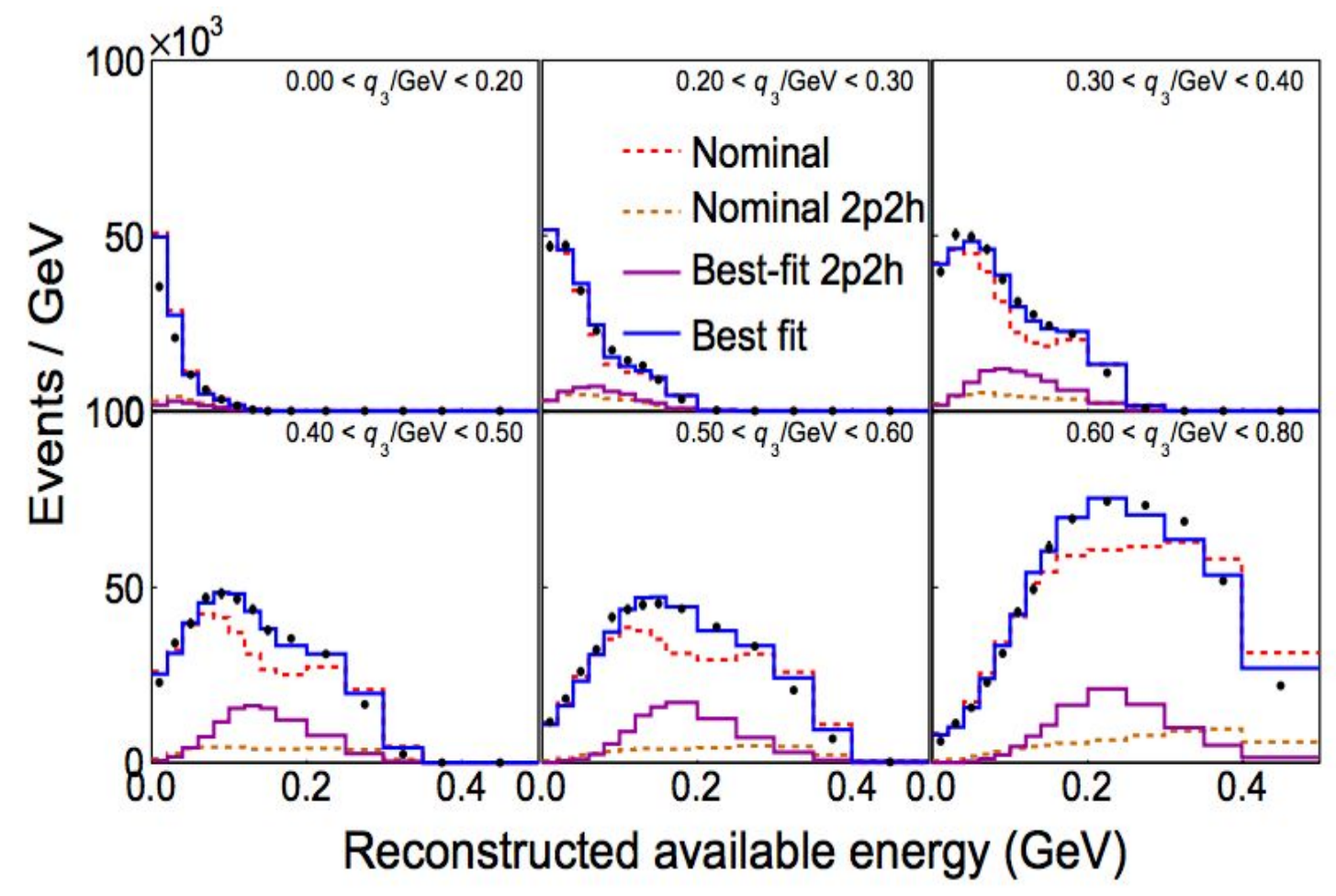

Figure 78: Effect of the 2-dimensional fitting in Fig. 77 on available energy distribution. Blue solid shows MC after applying the fit, comparing orange dashed line without the fit, , which corresponds to the solid curve in Fig. 76. (Taken from [14]) 

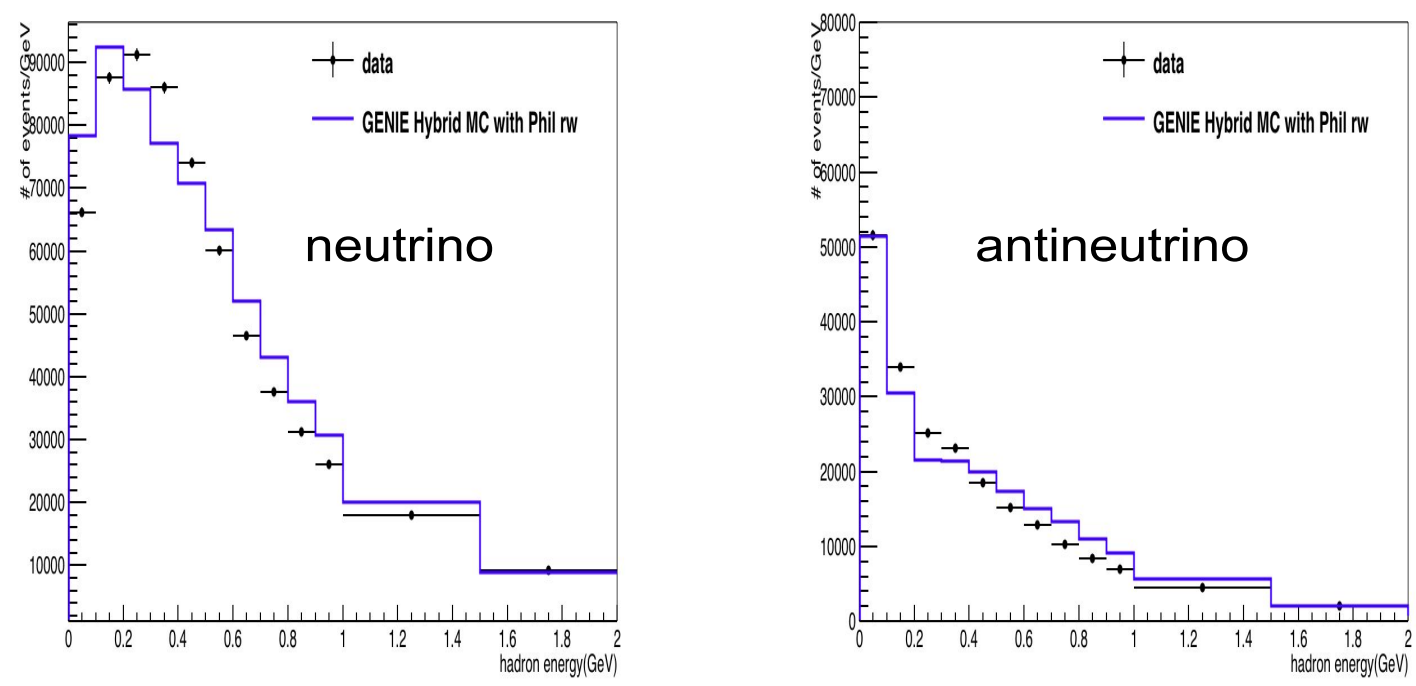

Figure 79: Remaining difference in hadron energy distributions between data and simulation after applying the 2-dimensional fitting for neutrino (left) and antineutrino (right) samples. 

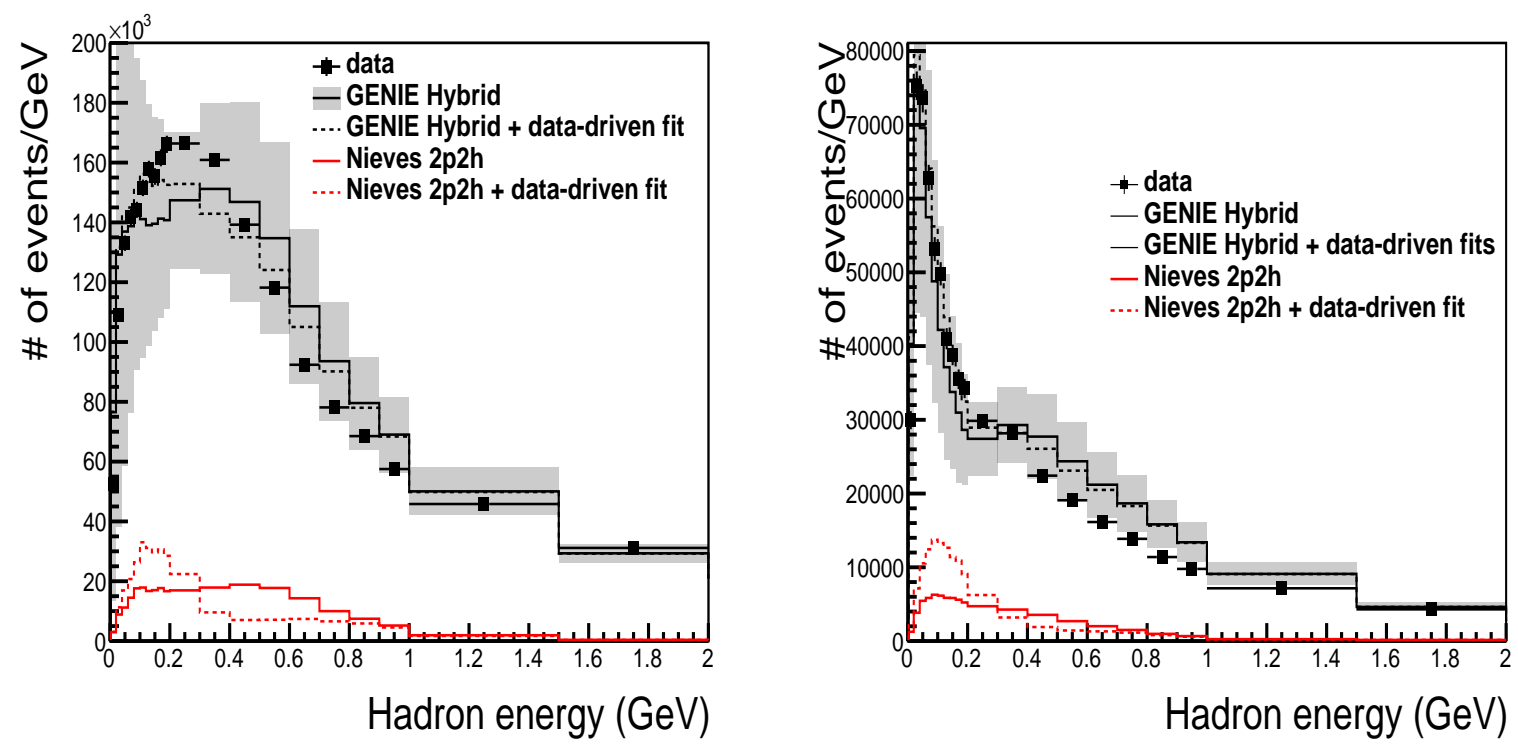

Figure 80: Data MC comparison of hadron energy after with and without data-driven fits. Left is for neutrino, Right is for antineutrino

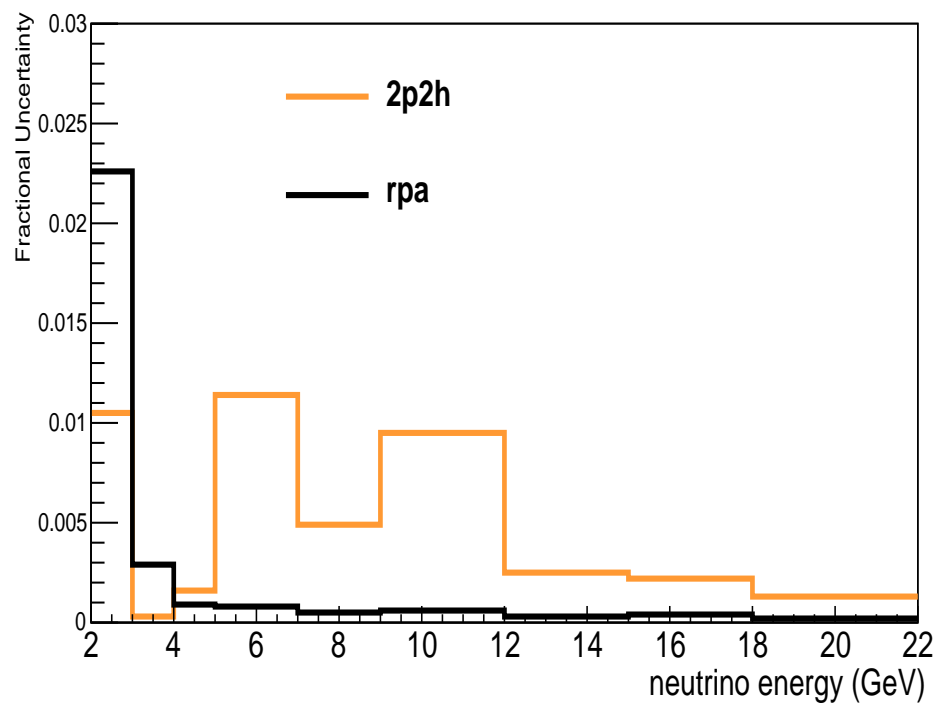

Figure 81: Effect of $2 \mathrm{p} 2 \mathrm{~h}$ reweighting on neutrino cross section. 


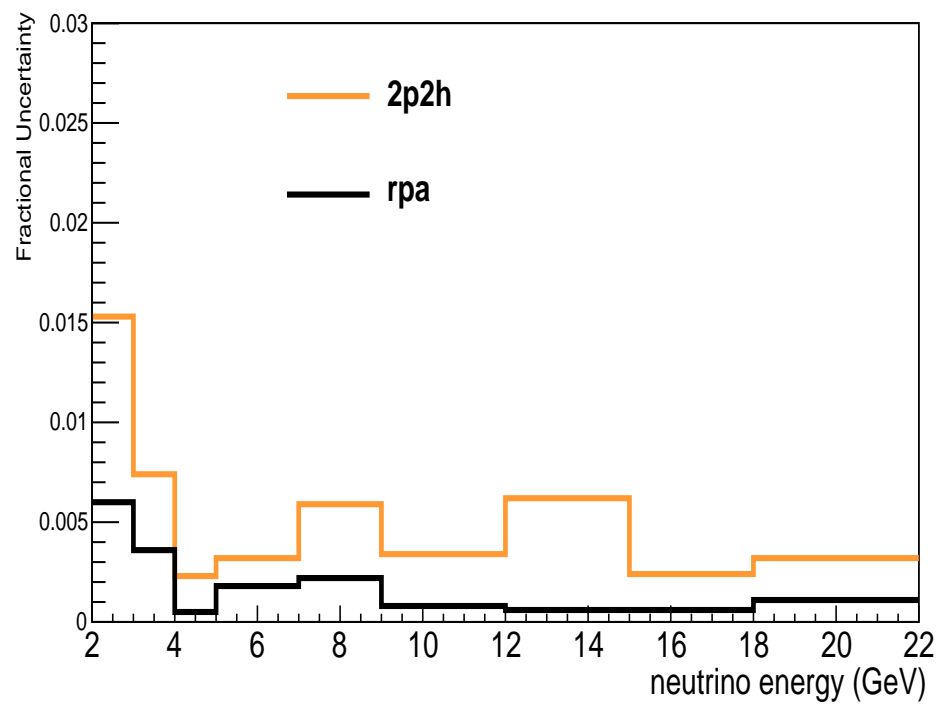

Figure 82: Effect of $2 \mathrm{p} 2 \mathrm{~h}$ reweighting on antineutrino cross section.

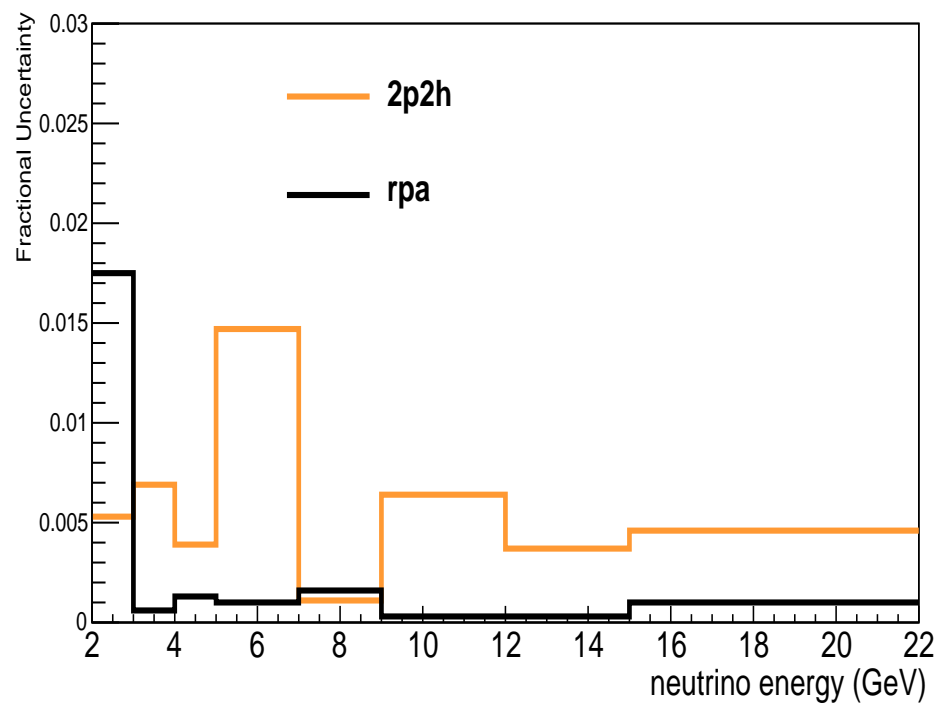

Figure 83: Effect of $2 \mathrm{p} 2 \mathrm{~h}$ reweighting on the ratio, $r$. 


\subsection{SUMMARY OF SYSTEMATIC UNCERTAINTIES}

Summary of systematic uncertainties evaluated are shown Fig. 84,Fig. 85 and Fig. 86. Numbers are available in Tab. 43, Tab. 44 and Tab. 45, for neutrino, antineutrino cross sections and the ratio, $r$, respectively.

The uncertainty of the neutrino cross section has a minimum between 12 and $22 \mathrm{GeV}$, which is due to the normalization method. At this value, we pin the cross section to match external world data. The normalization uncertainty is constant with energy and also sizable (3.58\%) for neutrino and antineutrino cross sections, while for the ratio, $r$, it cancels.

The uncertainty is dominated by cross section model uncertainties at low energy and normalization at higher energies. All systematic uncertainties other than normalization uncertainty decrease as neutrino energy increases, which is due to the external normalization. Similarly, for antineutrino cross section, cross section model also dominates at low energies, while uncertainty of normalization has the second largest contribution. For the ratio, $r$, many uncertainties cancel between neutrino and antineutrino cross sections, such as energy scales and normalization. The cross section model uncertainty dominates as well.

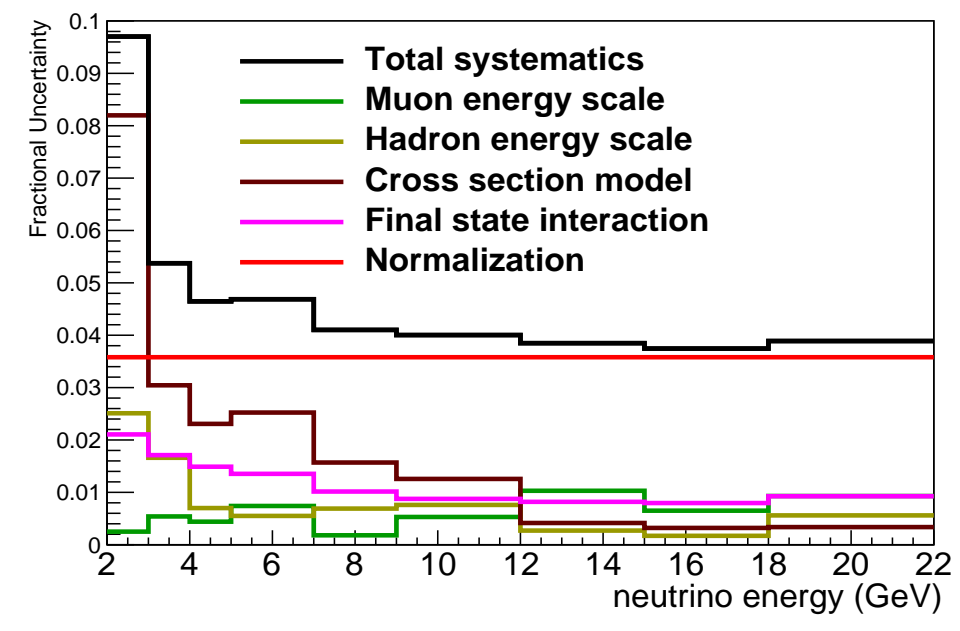

Figure 84: Error summary for neutrino cross section. External uncertainty is shown as the normalization curve. 


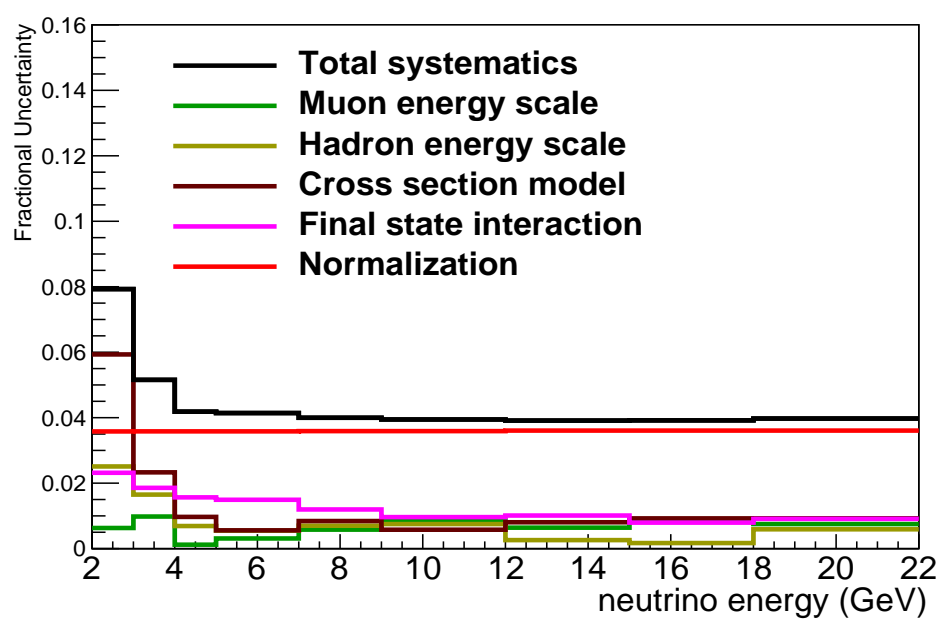

Figure 85: Error summary for antineutrino cross section. External uncertainty and $G_{c o r r}\left(\nu_{0}\right)$ uncertainty are added in quadrature and shown as the normalization curve.

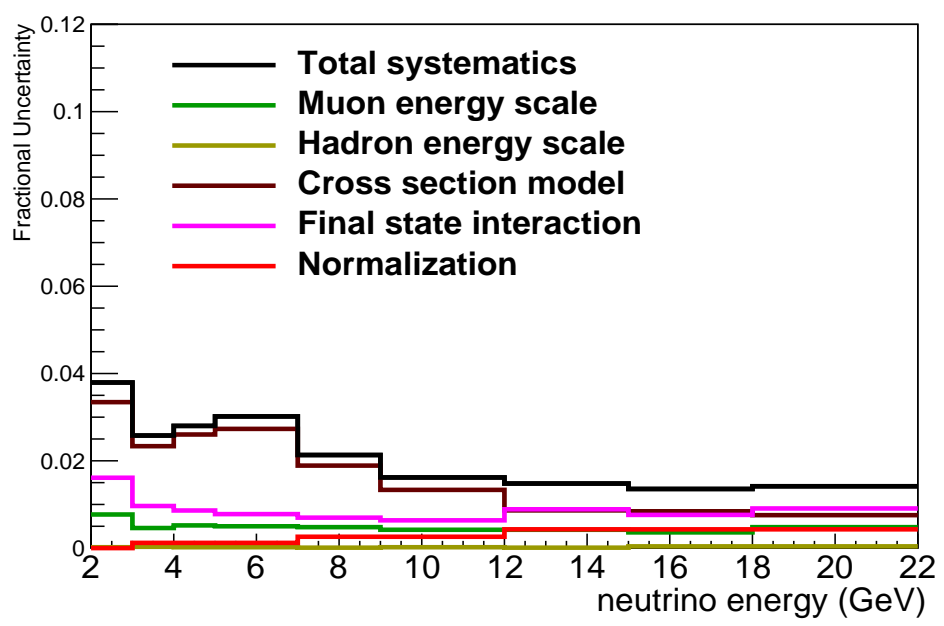

Figure 86: Error summary for the ratio, $r . G_{c o r r}\left(\nu_{0}\right)$ uncertainty is shown as the normalization curve. 


\begin{tabular}{|c|c|c|c|c|c|c|c|c|c|}
\hline \hline Source & 2.5 & 3.5 & 4.5 & 6 & 8 & 10.5 & 13.5 & 16.5 & 20 \\
& $\mathrm{GeV}$ & $\mathrm{GeV}$ & $\mathrm{GeV}$ & $\mathrm{GeV}$ & $\mathrm{GeV}$ & $\mathrm{GeV}$ & $\mathrm{GeV}$ & $\mathrm{GeV}$ & $\mathrm{GeV}$ \\
\hline Muon Energy Scale & 0.003 & 0.005 & 0.004 & 0.007 & 0.002 & 0.005 & 0.010 & 0.006 & 0.009 \\
Hadron Energy Scale & 0.025 & 0.017 & 0.007 & 0.006 & 0.007 & 0.007 & 0.003 & 0.002 & 0.005 \\
shower cleaning & 0.018 & 0.009 & 0.007 & 0.003 & 0.001 & 0.001 & 0.001 & 0.001 & 0.004 \\
FSI & 0.021 & 0.017 & 0.015 & 0.014 & 0.010 & 0.009 & 0.008 & 0.008 & 0.009 \\
Normalization & 0.036 & 0.036 & 0.036 & 0.036 & 0.036 & 0.036 & 0.036 & 0.036 & 0.036 \\
Cross section model & 0.082 & 0.030 & 0.023 & 0.025 & 0.016 & 0.013 & 0.004 & 0.003 & 0.003 \\
\hline Total & 0.097 & 0.054 & 0.046 & 0.047 & 0.041 & 0.040 & 0.038 & 0.037 & 0.039 \\
\hline
\end{tabular}

Table 43: Systematic uncertainty breakdown for the neutrino cross section.

Systematic uncertainties for flux measurements are shown in Fig. 87 and Fig. 88. At low energy, the uncertainties of both neutrino and antineutrino flux are dominated by muon energy scale, FSI and cross section model uncertainty. 


\begin{tabular}{|c|c|c|c|c|c|c|c|c|c|}
\hline \hline Source & $\begin{array}{c}2.5 \\
\mathrm{GeV}\end{array}$ & $\begin{array}{c}3.5 \\
\mathrm{GeV}\end{array}$ & $\begin{array}{c}4.5 \\
\mathrm{GeV}\end{array}$ & $\begin{array}{c}6 \\
\mathrm{GeV}\end{array}$ & $\begin{array}{c}8 \\
\mathrm{GeV}\end{array}$ & $\begin{array}{c}10.5 \\
\mathrm{GeV}\end{array}$ & $\begin{array}{c}13.5 \\
\mathrm{GeV}\end{array}$ & $\begin{array}{c}16.5 \\
\mathrm{GeV}\end{array}$ & $\begin{array}{c}20 \\
\mathrm{GeV}\end{array}$ \\
\hline Muon Energy Scale & 0.006 & 0.010 & 0.001 & 0.003 & 0.006 & 0.009 & 0.006 & 0.009 & 0.007 \\
Hadron Energy Scale & 0.025 & 0.017 & 0.007 & 0.005 & 0.007 & 0.007 & 0.003 & 0.002 & 0.006 \\
shower cleaning & 0.017 & 0.011 & 0.009 & 0.012 & 0.001 & 0.000 & 0.003 & 0.001 & 0.004 \\
Background & 0.000 & 0.000 & 0.000 & 0.001 & 0.003 & 0.003 & 0.003 & 0.002 & 0.001 \\
FSI & 0.023 & 0.019 & 0.016 & 0.015 & 0.012 & 0.010 & 0.010 & 0.008 & 0.009 \\
Normalization & 0.036 & 0.036 & 0.036 & 0.036 & 0.036 & 0.036 & 0.039 & 0.039 & 0.039 \\
Cross section model & 0.059 & 0.023 & 0.010 & 0.006 & 0.008 & 0.006 & 0.008 & 0.009 & 0.009 \\
\hline Total on data & 0.079 & 0.052 & 0.042 & 0.041 & 0.040 & 0.039 & 0.039 & 0.039 & 0.040 \\
\hline
\end{tabular}

Table 44: Systematic uncertainty breakdown for the antineutrino cross section.

\begin{tabular}{|c|c|c|c|c|c|c|c|c|c|}
\hline \hline Source & 2.5 & 3.5 & 4.5 & 6 & 8 & 10.5 & 13.5 & 16.5 & 20 \\
$\mathrm{GeV}$ & $\mathrm{GeV}$ & $\mathrm{GeV}$ & $\mathrm{GeV}$ & $\mathrm{GeV}$ & $\mathrm{GeV}$ & $\mathrm{GeV}$ & $\mathrm{GeV}$ & $\mathrm{GeV}$ \\
\hline Muon Energy Scale & 0.008 & 0.005 & 0.005 & 0.005 & 0.005 & 0.004 & 0.004 & 0.004 & 0.005 \\
Hadron Energy Scale & 0.000 & 0.000 & 0.000 & 0.000 & 0.000 & 0.000 & 0.000 & 0.000 & 0.000 \\
Shower cleaning & 0.001 & 0.002 & 0.002 & 0.009 & 0.001 & 0.000 & 0.001 & 0.000 & 0.000 \\
Background & 0.000 & 0.000 & 0.000 & 0.001 & 0.003 & 0.004 & 0.003 & 0.002 & 0.001 \\
FSI & 0.016 & 0.010 & 0.009 & 0.008 & 0.007 & 0.006 & 0.009 & 0.008 & 0.009 \\
Cross section model & 0.033 & 0.023 & 0.026 & 0.027 & 0.019 & 0.013 & 0.009 & 0.008 & 0.008 \\
\hline Total on r & 0.038 & 0.026 & 0.028 & 0.030 & 0.021 & 0.016 & 0.015 & 0.014 & 0.014 \\
\hline
\end{tabular}

Table 45: Systematic uncertainty breakdown for the ratio, $r$. 


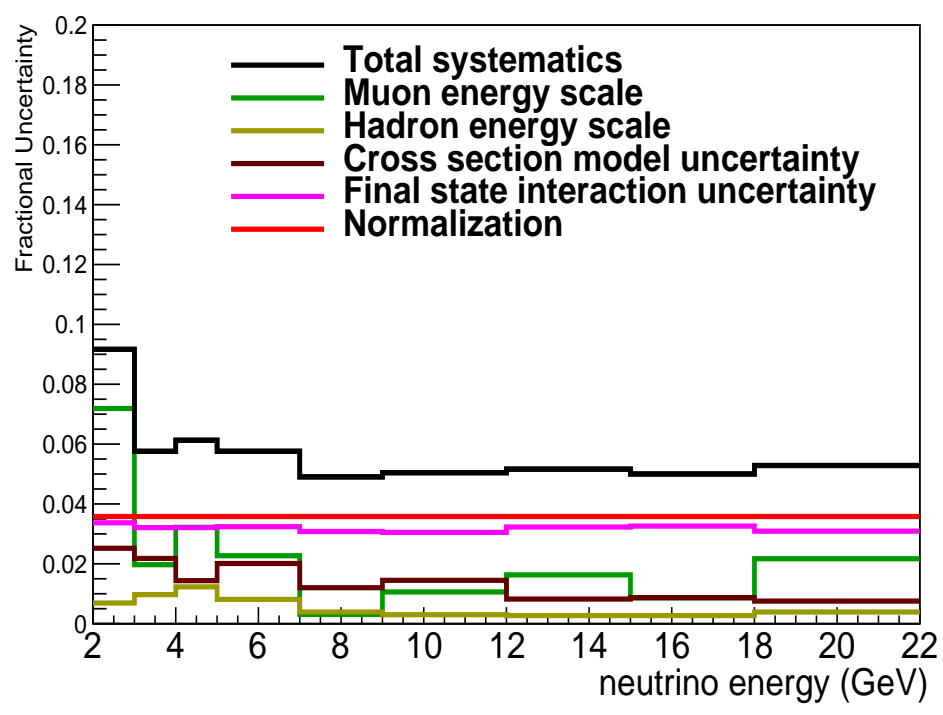

Figure 87: Error summary ofor FHC neutrino flux. External uncertainty is shown as the normalization curve.

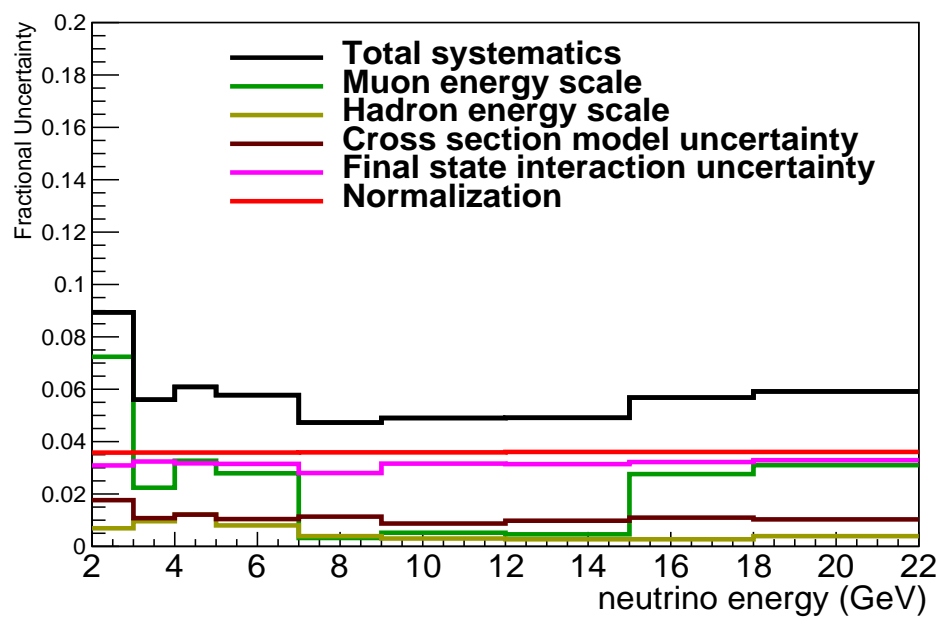

Figure 88: Error summary for RHC antineutrino flux. External uncertainty and $G_{c o r r}\left(\nu_{0}\right)$ uncertainty are added in quadrature and shown as the normalization curve. 


\subsection{RESULTS}

In this chapter, we present the results of measured quantities, cross sections, fluxes and the cross section ratio, $r$. We also show the comparison of kinematic variables between data and simulated MC samples, as well as some cross check studies.

\subsection{CROSS SECTIONS AND THE RATIO}

Primary results presented here use GENIE Hybrid model corrections, as described in Sec. 6.3. Extracted quantities using NuWro-based corrections are discussed in Sec. 8.4.1 as a cross-check.

Fig. 89 shows the extracted neutrino cross section. Measured cross sections agree with GENIEHybrid model curve above $7 \mathrm{GeV}$. In 3-5 GeV, data is about $2 \sigma$ lower than the model curve. Fig. 90 shows the extracted antineutrino cross section, which agrees with model curve within the precision of the data. Fig. 91 shows the extracted cross section ratio, $r$, which is above the model curve at low energy and favors a flatter shape than model. Tab. 47, Tab. 48 and Tab. 46 show the measured quantities, statistical and systematic errors.

In addition to the statistical error of binned data samples, there is additional statistical error introduced by the normalization procedure. These include two sources. One comes from the statistical error of unnormalized neutrino cross section in $12-22 \mathrm{GeV}$, which propagates into antineutrino cross section, flux and cancels for the ratio, $r$. The other is from the statistical error of the $\alpha\left(\nu_{0}\right)$ correction (defined in Sec. 6.5.1) for antineutrino cross section and the ratio, $r$ (which

comes from the unnormalized antineutrino cross section in 12-22 GeV). A summary is shown in Tab. 26. These errors are added in quadrature and included in the total statistical error for each measured quantity. 


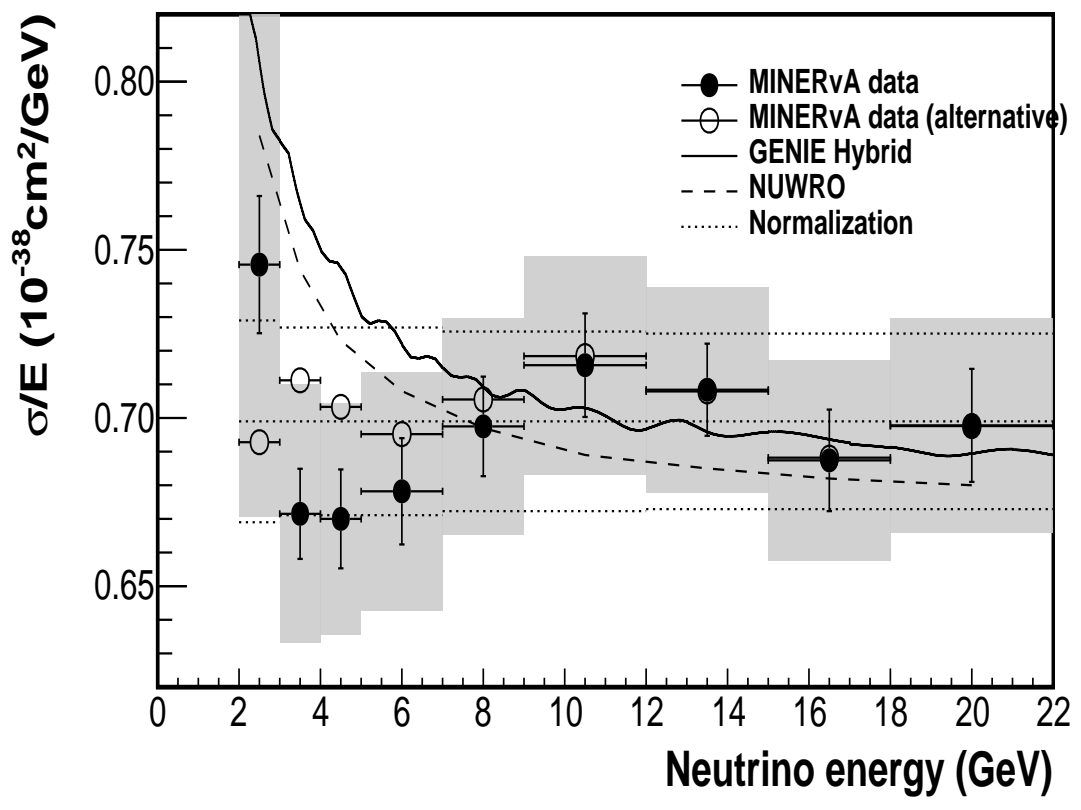

Figure 89: Measured neutrino cross section using GENIE-Hybrid corrections (solid points), comparing with GENIE Hybrid model curve. Error bar includes statistical error only and the shaded band stands for total uncertainty. Measured neutrino cross section using NuWro-based corrections (open circles) will be discussed in Sec. 8.4.1.

A comparison of statistical error and systematic error is shown in Fig. 92 , 93, 94. Uncertainty of neutrino cross section is dominated by systematic uncertainty, while the uncertainty of antineutrino cross section and $r$ are dominated by statistical error.

\subsection{FLUX}

The measured normalized FHC-mode neutrino (RHC antineutrino) low- $\nu$ flux is shown in Fig. 95 (Fig. 96). Tab. 49 and Tab. 50 show the measured fluxes and their statistical and systematic error. They are compared with the input simulated fluxes which have been discussed in Sec. 2.1. The 


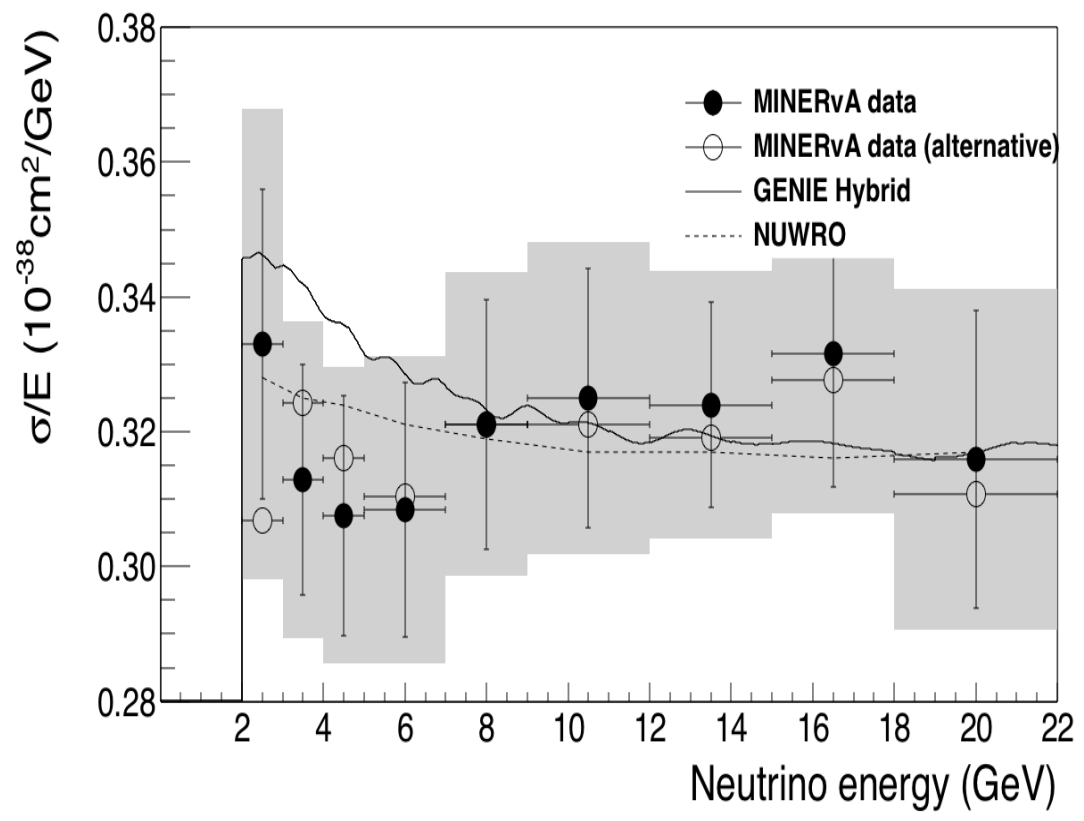

Figure 90: Measured antineutrino cross section using GENIE-Hybrid corrections (solid points), comparing with GENIE Hybrid model curve. Error bar includes statistical error only and the shaded band stands for total uncertainty. Measured neutrino cross section using NuWro-based corrections (open circles) will be discussed in Sec. 8.4.1.

measured low- $\nu$ flux has better precision for neutrinos (by $30 \%$ above $3 \mathrm{GeV}$ ) and comparable for antineutrinos to that from simulation. The extracted low- $\nu$ neutrino (antineutrino) flux agrees with the simulated flux within the precision of the data, neutrino (antineutrino) data prefers a smaller (larger) flux below $3 \mathrm{GeV}$. While above $7 \mathrm{GeV}$, both neutrino and antineutrino fluxes are above the simulated fluxes. 


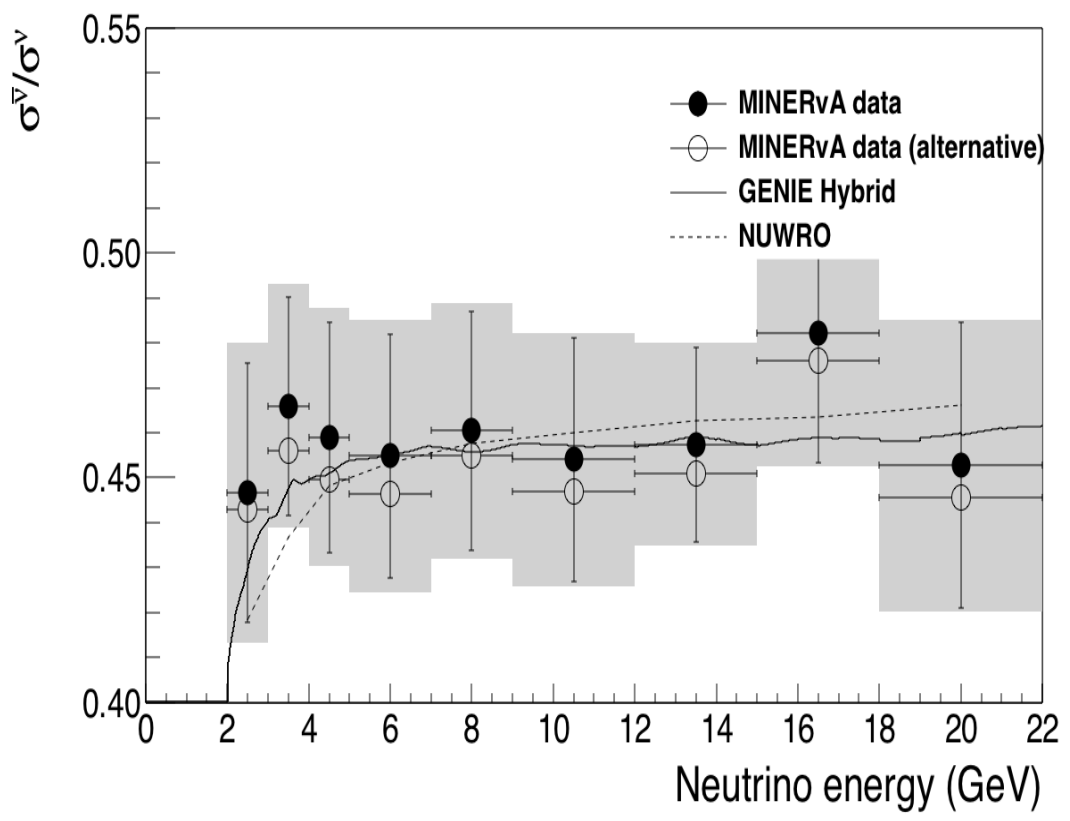

Figure 91: Measured cross section ratio, $r$, using GENIE-Hybrid corrections (solid points), comparing with GENIE Hybrid model curve. Error bar includes statistical error only and the shaded band stands for total uncertainty. Measured neutrino cross section using NuWro-based corrections (open circles) will be discussed in Sec. 8.4.1.

\subsection{COMPARISON OF DATA WITH MC MODEL}

In this section, we show the comparison of data and $\mathrm{MC}$ simulation for reconstructed neutrino energy, muon energy, muon angle and hadron energy.

Corrections are applied to MC simulation samples to account for trc=acking efficiency in matching tracks from MINER $\nu \mathrm{A}$ into MINOS ND. Two tracking efficiency corrections are evaluated for MINER $\nu \mathrm{A}$ and MINOS separately. MINER $\nu \mathrm{A}$ muon tracking efficiency correction [68] is obtained using the sample of events which have a track at MINOS front face (the upstream end), by calculating the fraction of events which point back to a found track in the MINER $\nu$ A fiducial region. This correction is 0.995 , and is the same for all simulated MC samples. The MINOS muon 


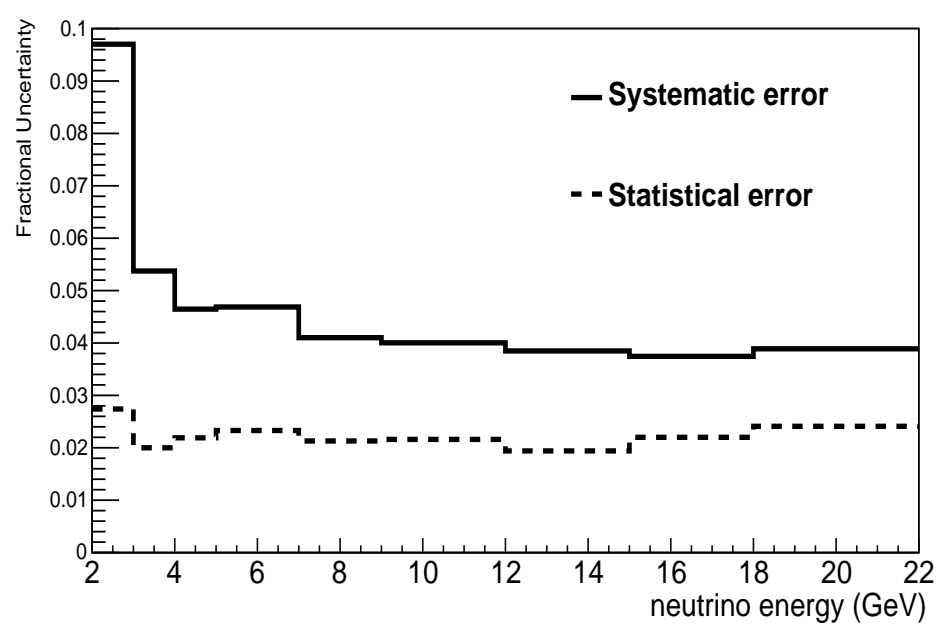

Figure 92: Statistical and systematic errors comparison of neutrino cross section.

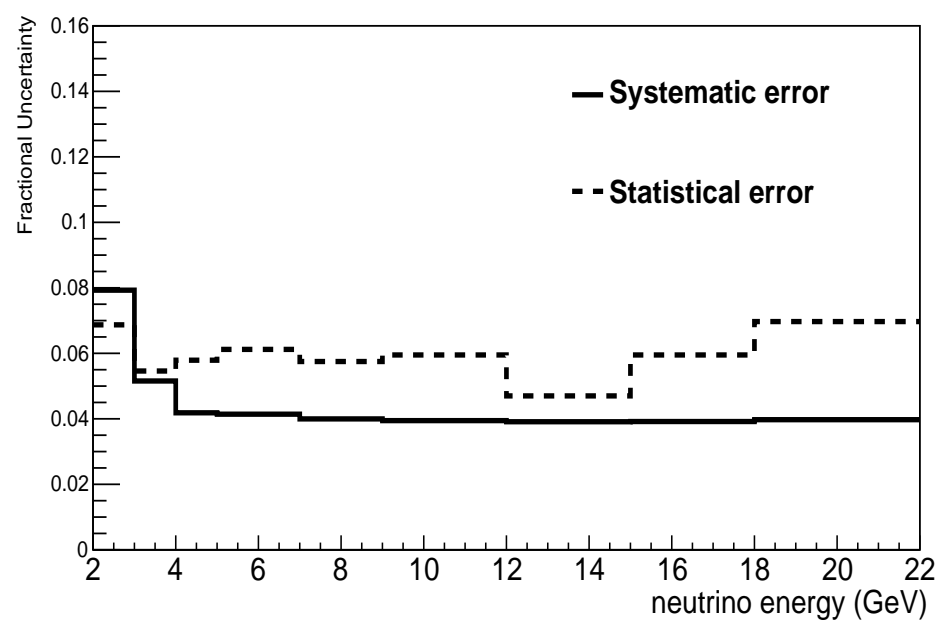

Figure 93: Statistical and systematic errors comparison of antineutrino cross section.

tracking efficiency correction [68] is shown in Tab. 51. The sample is divided into high momentum and low momentum samples and the correction is determined by identifying events with a track in MINER $\nu$ A that point to the MINOS fiducial area. MINOS tracking efficiency correction is the 


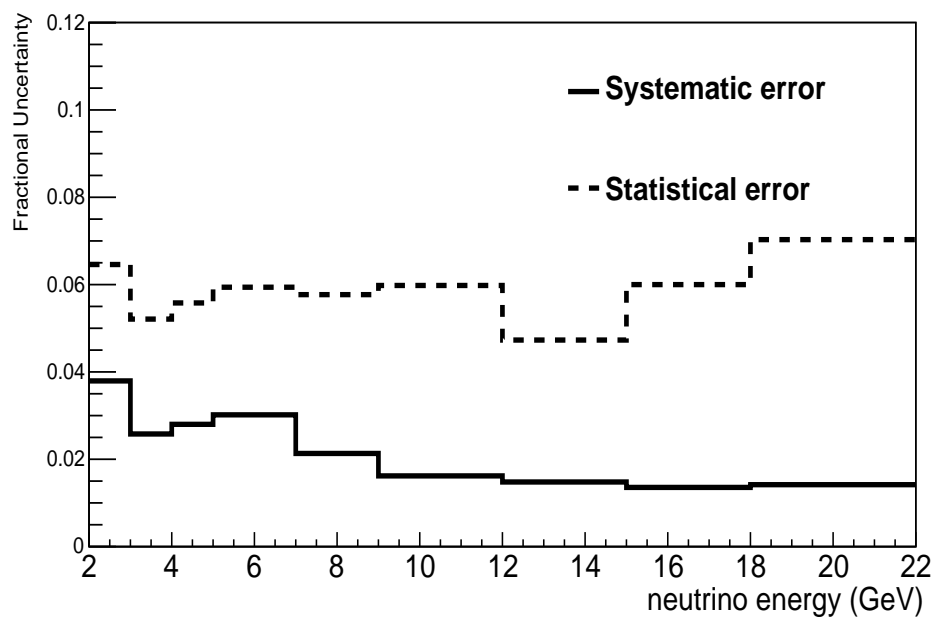

Figure 94: Statistical and systematic errors comparison of the ratio, $r$.

fraction of these events which have a track in MINOS.

For both data and $\mathrm{MC}$, we are selecting events which pass the reconstructed cuts described in Sec. 5.1. In addition, we require minimum and maximum reconstructed neutrino energy cut: $2<E_{\nu}<22 \mathrm{GeV}$, which allows only events used to extract cross sections and fluxes below 22 $\mathrm{GeV}$.

In order to correct the input flux, a low- $\nu$ flux reweighting function based on our measured low$\nu$ flux is defined as $f^{\nu}\left(E_{\nu}\right)$ for FHC neutrinos $\left(f^{\bar{\nu}}\left(E_{\nu}\right)\right.$ for RHC antineutrinos). It is the ratio of the low- $\nu$ flux extracted from data and MC input flux in Fig. 8.2, and binned in true neutrino energy. Fig. 97 shows the low- $\nu$ flux reweighting function for FHC neutrinos and RHC antineutrinos. The simulated events are reweighted by using low- $\nu$ flux reweighting functions. This reweighting is performed for true neutrino energy $2<E_{\nu}<50 \mathrm{GeV}$.

Fig. 98 shows the distributions of neutrino energy for inclusive sample of FHC neutrinos and RHC antineutrinos. After applying low- $\nu$ flux reweighting function, data and MC simulation agreement is improved, especially above $7 \mathrm{GeV}$. The error bands includes the statistical error of low- $\nu$ flux reweighting and total systematic error (uncertainty of external normalization not included). 


\begin{tabular}{|c|c|c|c|c|}
\hline \hline $\mathrm{E}(\mathrm{GeV})$ & $r$ & stat. err. & syst. err. & total err \\
\hline 2.5 & 0.447 & 0.065 & 0.038 & 0.075 \\
3.5 & 0.466 & 0.052 & 0.026 & 0.058 \\
4.5 & 0.459 & 0.056 & 0.028 & 0.062 \\
6.0 & 0.455 & 0.059 & 0.030 & 0.067 \\
8.0 & 0.460 & 0.058 & 0.021 & 0.062 \\
10.5 & 0.454 & 0.060 & 0.016 & 0.062 \\
13.5 & 0.457 & 0.047 & 0.015 & 0.050 \\
16.5 & 0.482 & 0.060 & 0.013 & 0.062 \\
20.0 & 0.453 & 0.070 & 0.014 & 0.072 \\
\hline \hline
\end{tabular}

Table 46: The ratio, $r$, and its statistical, systematic and total error. Errors are fractional.

Fig. 99 shows the distributions of muon energy for inclusive sample of FHC neutrinos and RHC antineutrinos. After applying low- $\nu$ flux reweighting function, data and MC simulation agreement is improved (for neutrinos above $5 \mathrm{GeV}$ and for antineutrino in $5-12 \mathrm{GeV}$ and $18-22 \mathrm{GeV}$ ).

Fig. 100 shows the distributions of muon angle w.r.t. beam for inclusive sample of FHC neutrinos and RHC antineutrinos. The small angle $(0.058 \mathrm{rad})$ between the beam and $\mathrm{z}$-axis of the detector is accounted for. low- $\nu$ flux reweighting improves the agreement for wider muon angle.

Comparison of hadron energy distributions for FHC neutrinos and RHC antineutrinos are shown in different neutrino energy bins. Fig. 101, 102, 103 and 104 show the comparison for samples with neutrino energy in the region of $2<E_{\nu}<3 \mathrm{GeV}, 3<E_{\nu}<4 \mathrm{GeV}, 4<E_{\nu}<5 \mathrm{GeV}$, $5<E_{\nu}<7 \mathrm{GeV}, 7<E_{\nu}<12 \mathrm{GeV}, 12<E_{\nu}<22 \mathrm{GeV}$ and $2<E_{\nu}<22 \mathrm{GeV}$, respectively. The black points show data and the red histograms show GENIE-Hybrid model with low- $\nu$ flux. For most of the energy bins, data points are within the MC simulation error band. For neutrinos in the energy region $2<E_{\nu}<3 \mathrm{GeV}$, data is above the upper edge of error band for $0.1<E_{\text {had }}<0.3 \mathrm{GeV}$. For antineutrinos data points are always lower than MC simulation and on the edge of the error band for $E_{\text {had }}>0.4 \mathrm{GeV}$. Hadron energy distributions for higher neutrino en- 


\begin{tabular}{|c|c|c|c|c|}
\hline \hline $\mathrm{E}(\mathrm{GeV})$ & $\sigma / E\left(10^{-38} \mathrm{~cm}^{2} / \mathrm{GeV}\right)$ & stat. err. & syst. err. & total err \\
\hline 2.5 & 0.746 & 0.027 & 0.097 & 0.101 \\
3.5 & 0.671 & 0.020 & 0.054 & 0.057 \\
4.5 & 0.670 & 0.022 & 0.046 & 0.051 \\
6.0 & 0.678 & 0.023 & 0.047 & 0.052 \\
8.0 & 0.697 & 0.021 & 0.041 & 0.046 \\
10.5 & 0.716 & 0.022 & 0.040 & 0.045 \\
13.5 & 0.708 & 0.019 & 0.038 & 0.043 \\
16.5 & 0.687 & 0.022 & 0.037 & 0.043 \\
20.0 & 0.698 & 0.024 & 0.039 & 0.046 \\
\hline
\end{tabular}

Table 47: Neutrino cross section its statistical, systematic and total error. Errors are fractional.

ergy bins show the similar difference between data and MC simulation. However, their systematic uncertainty of hadron energy is larger, the difference is always within the systematic uncertainty. 


\begin{tabular}{|c|c|c|c|c|}
\hline \hline $\mathrm{E}$ & $\sigma / \mathrm{E}\left(10^{-38} \mathrm{~cm}^{2} / \mathrm{GeV}\right)$ & stat. err. & syst. err. & total err \\
\hline 2.5 & 0.333 & 0.069 & 0.079 & 0.105 \\
3.5 & 0.313 & 0.055 & 0.052 & 0.075 \\
4.5 & 0.308 & 0.058 & 0.042 & 0.071 \\
6.0 & 0.308 & 0.061 & 0.041 & 0.074 \\
8.0 & 0.321 & 0.058 & 0.040 & 0.070 \\
10.5 & 0.325 & 0.060 & 0.039 & 0.071 \\
13.5 & 0.324 & 0.047 & 0.039 & 0.061 \\
16.5 & 0.331 & 0.060 & 0.039 & 0.071 \\
20.0 & 0.316 & 0.070 & 0.040 & 0.08 \\
\hline \hline
\end{tabular}

Table 48: Antineutrino cross section its statistical, systematic and total error. Errors are fractional.

\begin{tabular}{|c|c|c|c|c|}
\hline \hline $\mathrm{E}(\mathrm{GeV})$ & $\Phi(E)$ & stat. err. & syst. err. & total err \\
\hline 2.5 & 70.290 & 0.026 & 0.092 & 0.095 \\
3.5 & 78.716 & 0.019 & 0.058 & 0.061 \\
4.5 & 30.052 & 0.021 & 0.061 & 0.065 \\
6.0 & 9.557 & 0.022 & 0.058 & 0.062 \\
8.0 & 5.269 & 0.020 & 0.049 & 0.053 \\
10.5 & 3.136 & 0.020 & 0.050 & 0.054 \\
13.5 & 1.916 & 0.018 & 0.052 & 0.055 \\
16.5 & 1.173 & 0.020 & 0.050 & 0.054 \\
20.0 & 0.651 & 0.022 & 0.053 & 0.057 \\
\hline
\end{tabular}

Table 49: Neutrino flux in unit of neutrinos $/ \mathrm{GeV} / \mathrm{m}^{2} / 10^{6} \mathrm{pot}$ and its statistical, systematic and total error. Errors are fractional. 


\begin{tabular}{|c|c|c|c|c|}
\hline \hline $\mathrm{E}(\mathrm{GeV})$ & $\Phi(E)$ & stat. err. & syst. err. & total err \\
\hline 2.5 & 68.851 & 0.067 & 0.089 & 0.112 \\
3.5 & 66.833 & 0.053 & 0.056 & 0.077 \\
4.5 & 24.171 & 0.056 & 0.061 & 0.083 \\
6.0 & 6.676 & 0.059 & 0.058 & 0.082 \\
8.0 & 3.017 & 0.053 & 0.047 & 0.071 \\
10.5 & 1.625 & 0.055 & 0.049 & 0.074 \\
13.5 & 0.895 & 0.039 & 0.049 & 0.063 \\
16.5 & 0.437 & 0.050 & 0.057 & 0.076 \\
20.0 & 0.229 & 0.060 & 0.059 & 0.085 \\
\hline \hline
\end{tabular}

Table 50: Antineutrino flux in unit of neutrinos $/ \mathrm{GeV} / \mathrm{m}^{2} / 10^{6}$ pot and its statistical, systematic and total error. Errors are fractional. 


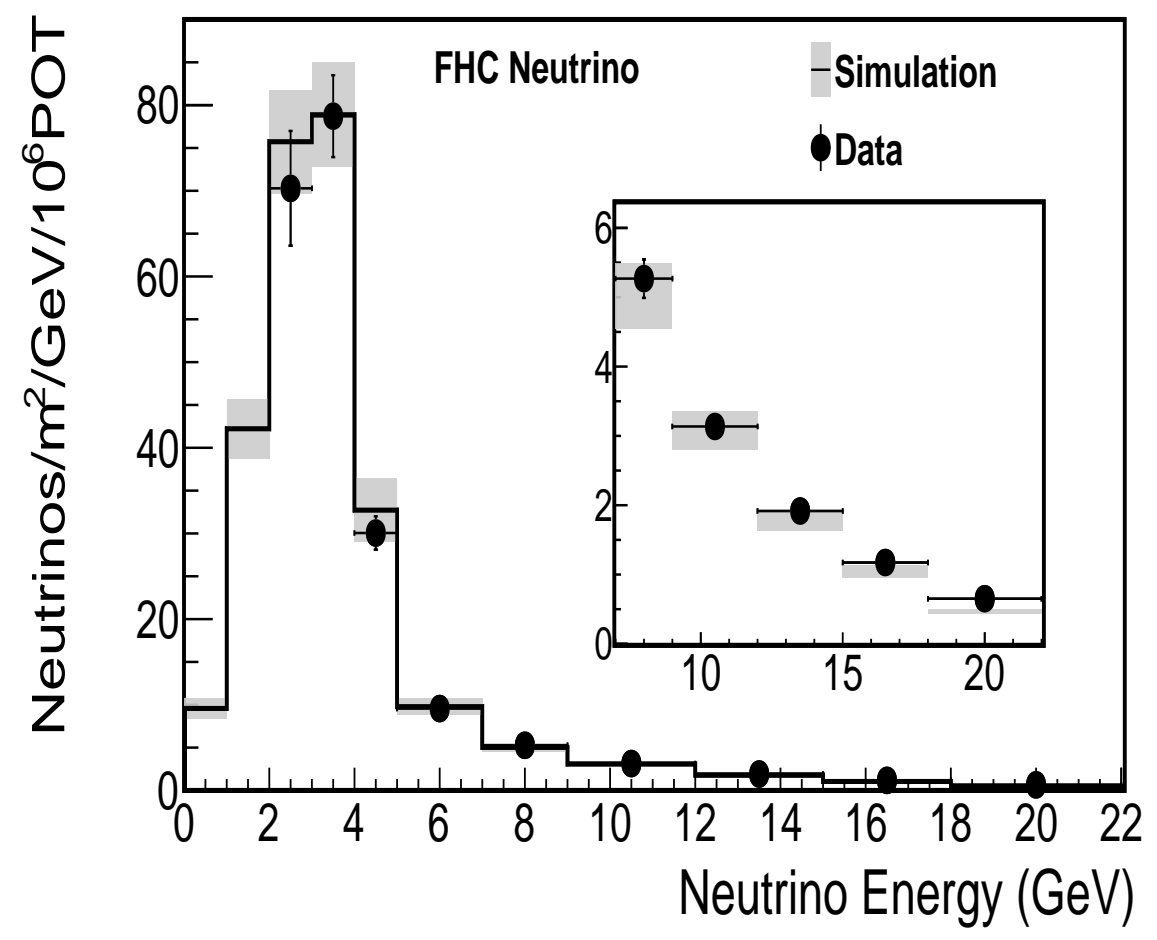

Figure 95: Measured FHC neutrino flux comparing with simulated MC flux. Error bars include statistical error and systematic uncertainty. 


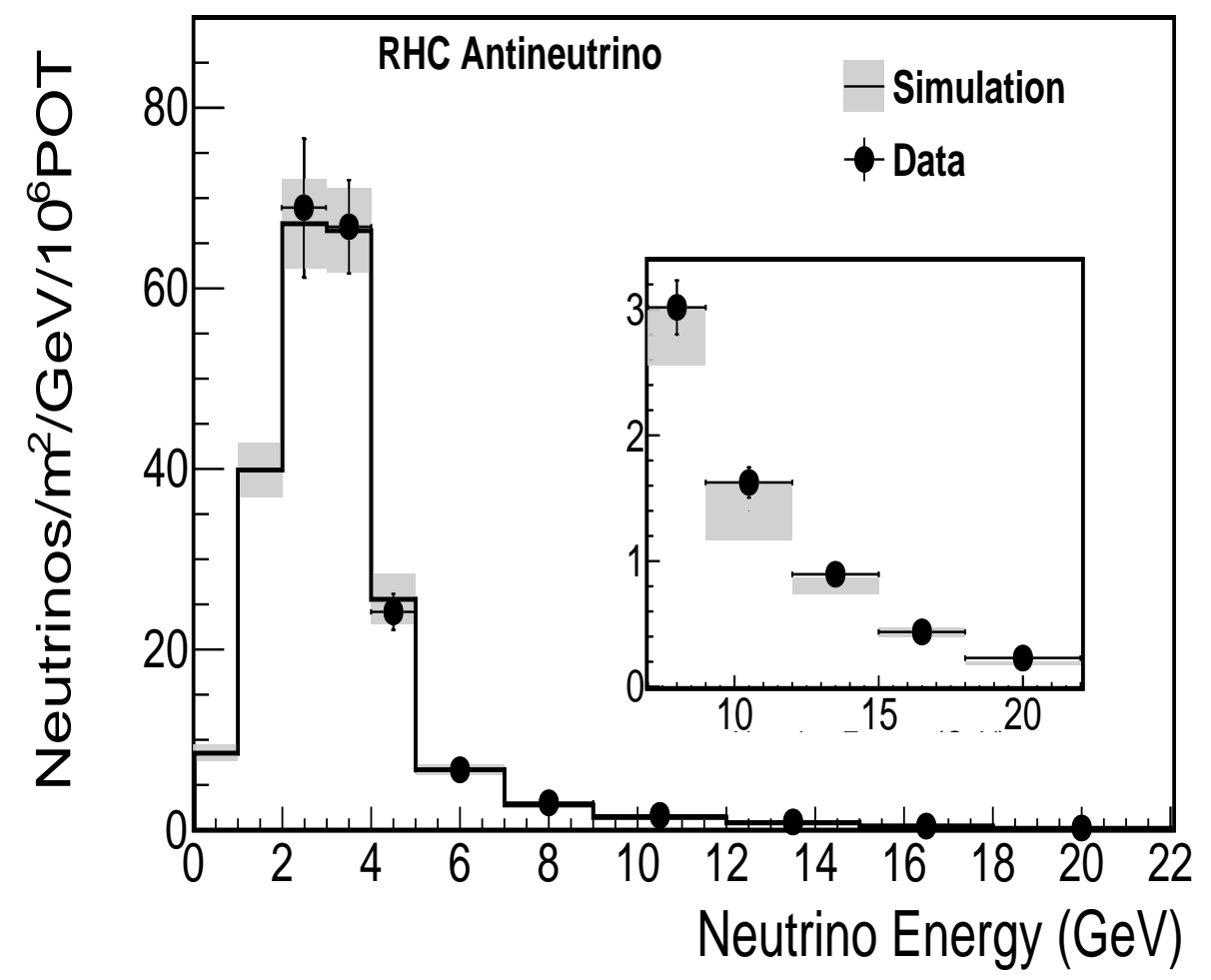

Figure 96: Measured RHC antineutrino flux comparing with simulated MC flux. Error bars include statistical error and systematic uncertainty.

\begin{tabular}{ccc}
\hline \hline playlist & $p_{\mu}<3 \mathrm{GeV}$ & $p_{\mu}>3 \mathrm{GeV}$ \\
\hline minerva1 & 0.963 & 0.990 \\
minerva5 & 0.975 & 0.995 \\
minerva7 & 0.975 & 0.995 \\
minerva9 & 0.972 & 0.996 \\
minerva13BCDE & 0.971 & 0.994 \\
\hline
\end{tabular}

Table 51: MINOS tracking efficiency correction. 


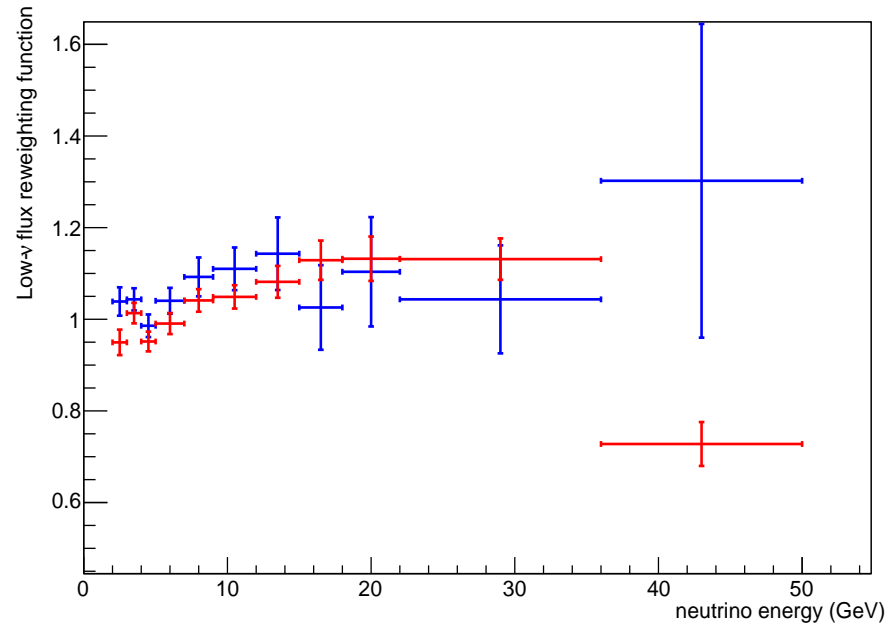

Figure 97: Low- $\nu$ flux reweighting function. Error bar includes statistical error as well as systematic uncertainties of extracted flux from data. 

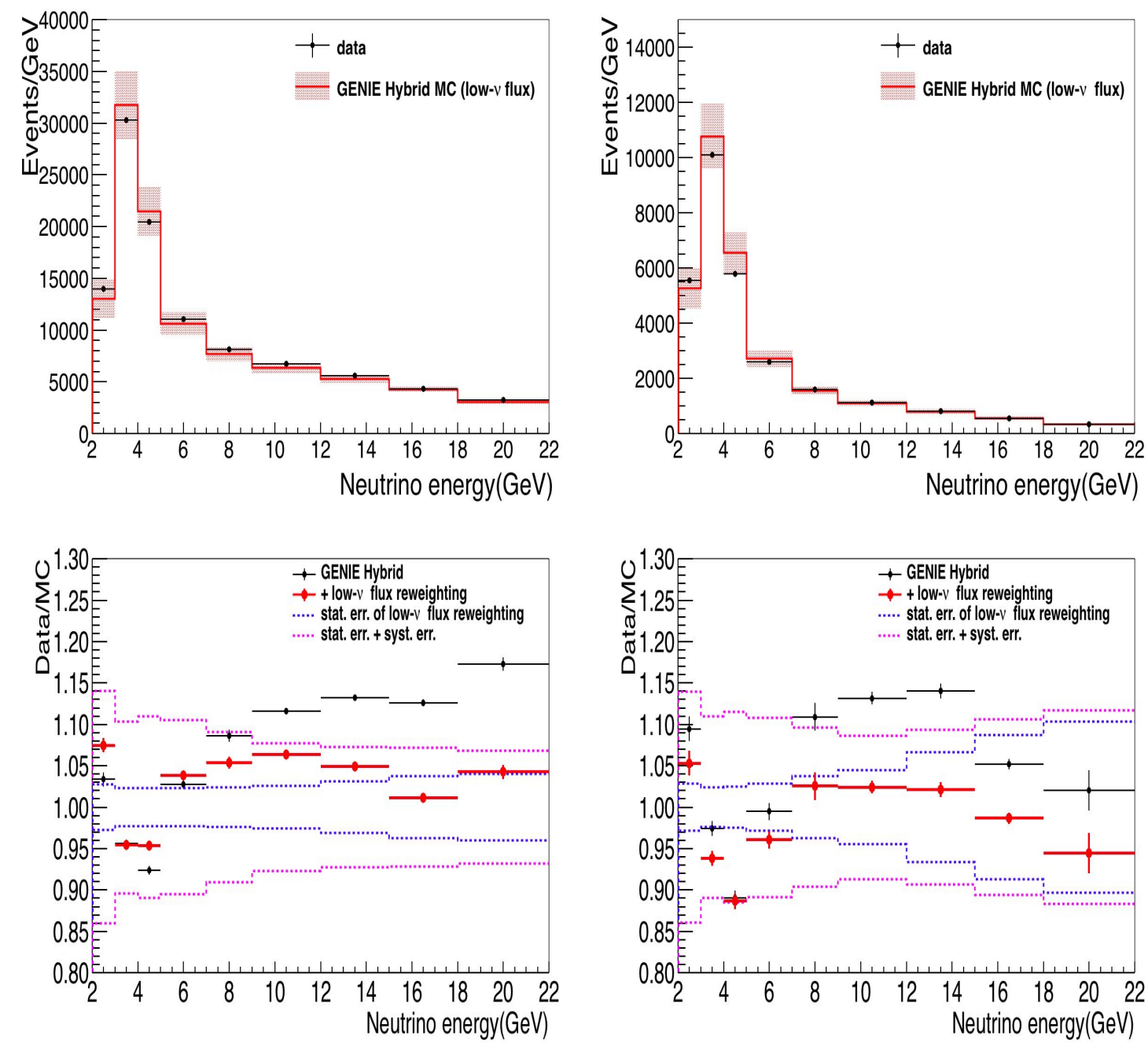

Figure 98: Comparison of data and MC simulation distributions of neutrino energy for FHC neutrinos (left) and RHC antineutrinos(right). Upper plots show the muon energy distributions. The points show data and histograms show GENIE-Hybrid model with low- $\nu$ flux. The error bands include the statistical error of low- $\nu$ flux reweighting and total systematic error (uncertainty of external normalization not included).Bottom plots show the ratio of data to MC simulation. Black points show the ratio of data to MC simulation with default flux (hadron production constrained flux shown in Fig. 14), the red points show the ratio with the low- $\nu$ flux reweighting. The blue band shows the statistical error of low- $\nu$ flux reweighting. The pink band shows the systematic error and statistical error of low- $\nu$ flux reweighting added in quadrature. All plots are POT normalized. 

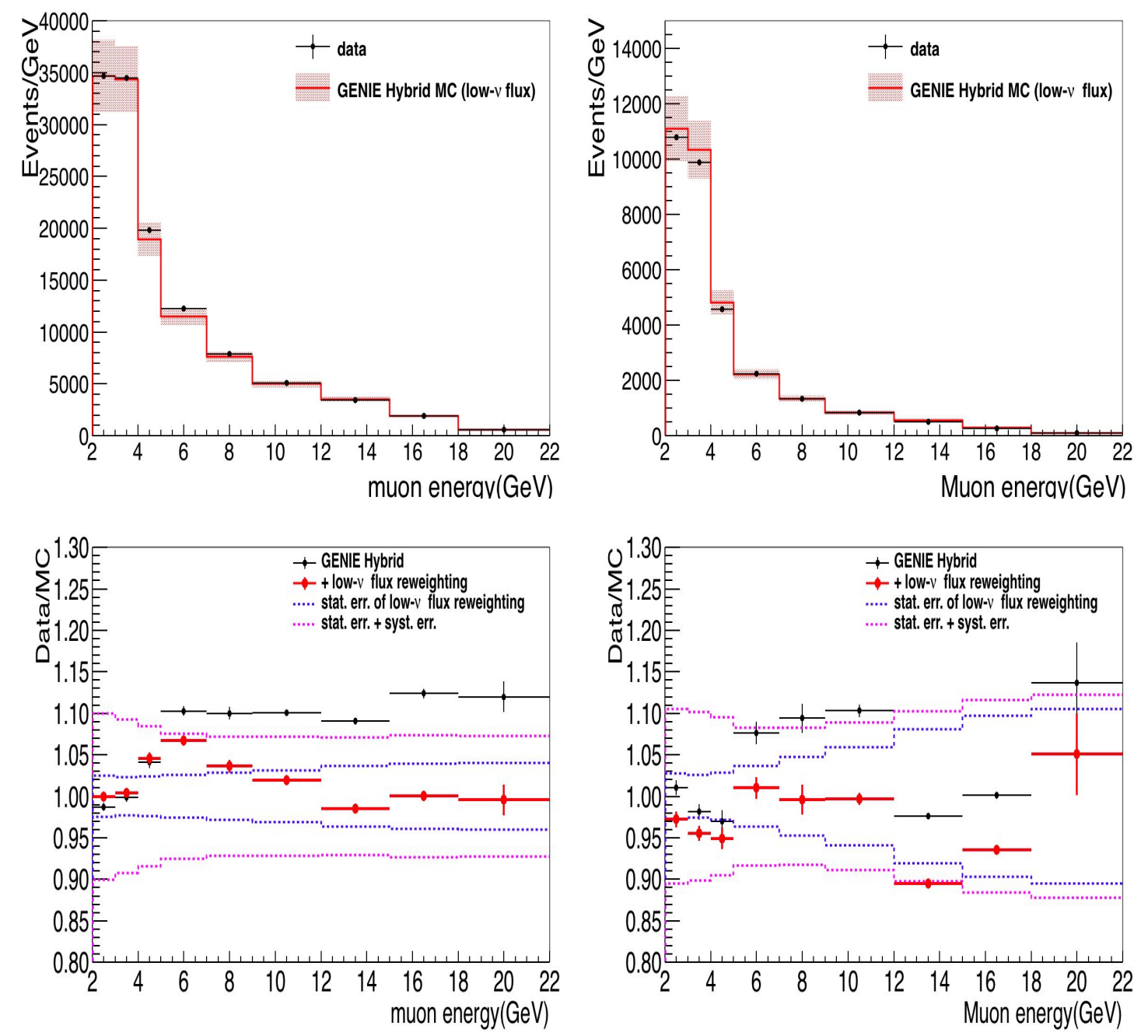

Figure 99: Comparison of data and MC simulation distributions of muon energy for FHC neutrinos (left) and RHC antineutrinos(right). Upper plots show the muon energy distributions. The points show data and histograms show GENIE-Hybrid model with low- $\nu$ flux. The error bands include the statistical error of low- $\nu$ flux reweighting and total systematic error (uncertainty of external normalization not included). Bottom plots show the ratio of data to MC simulation. Black points show the ratio of data to MC simulation with default flux (hadron production constrained flux shown in Fig. 14), the red points show the ratio with the low- $\nu$ flux flux reweighting. The blue band shows the statistical error of low- $\nu$ flux reweighting. The pink band shows the systematic error and statistical error of low- $\nu$ flux reweighting added in quadrature. All plots are POT normalized. 

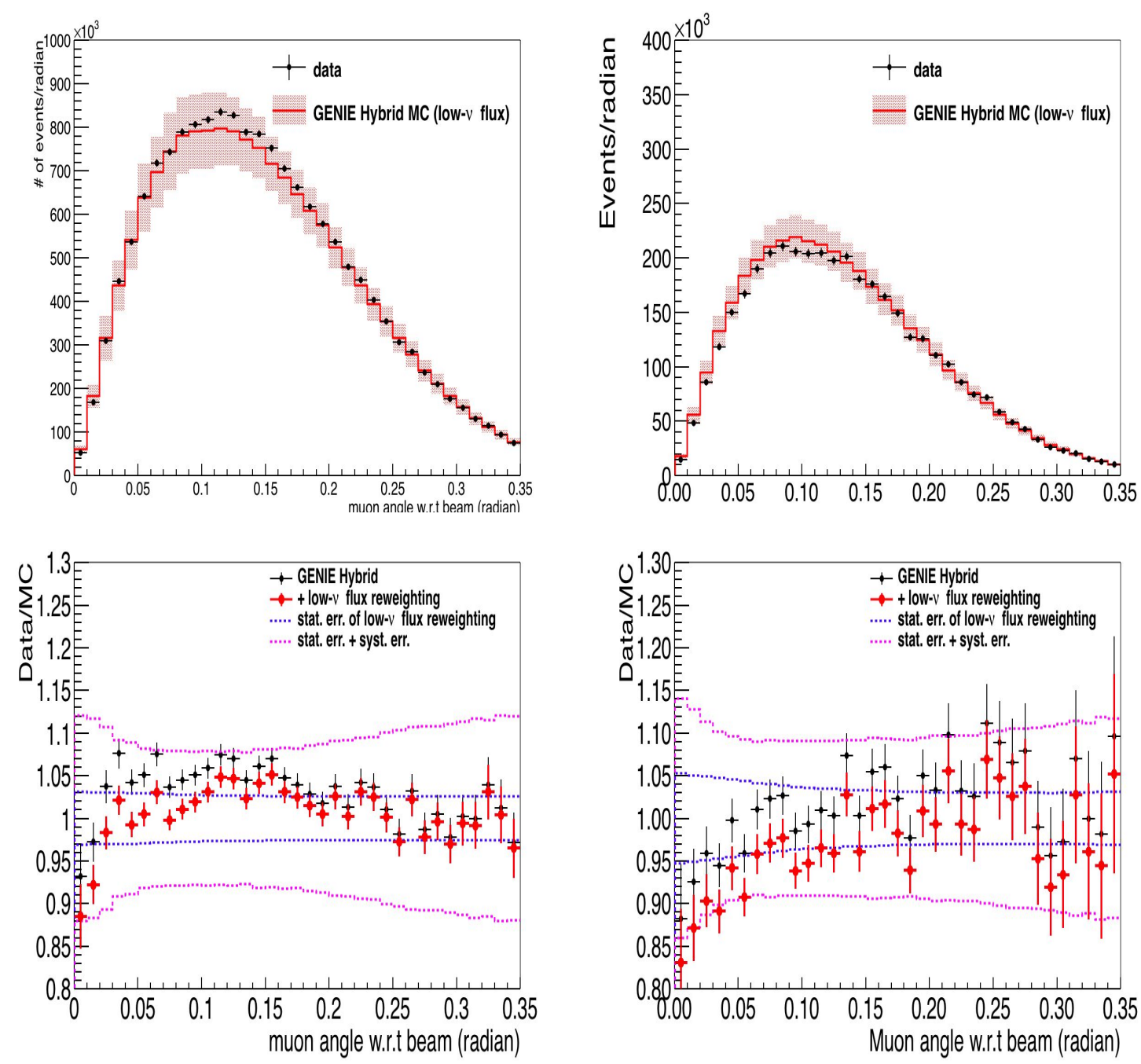

Figure 100: Comparison of data and MC simulation distributions of muon angle w.r.t beam for FHC neutrinos (left) and RHC antineutrinos(right). Upper plots show the muon energy distributions. The points show data and histograms show GENIE-Hybrid model with low- $\nu$ flux. The error bands include the statistical error of low- $\nu$ flux reweighting and total systematic error (uncertainty of external normalization not included).Bottom plots show the ratio of data to MC simulation. Black points show the ratio of data to MC simulation with default flux (hadron production constrained flux shown in Fig. 14), the red points show the ratio with the low- $\nu$ flux reweighting. The blue band shows the statistical error of low- $\nu$ flux reweighting. The pink band shows the systematic error and statistical error of low- $\nu$ flux reweighting added in quadrature. All plots are POT normalized. 

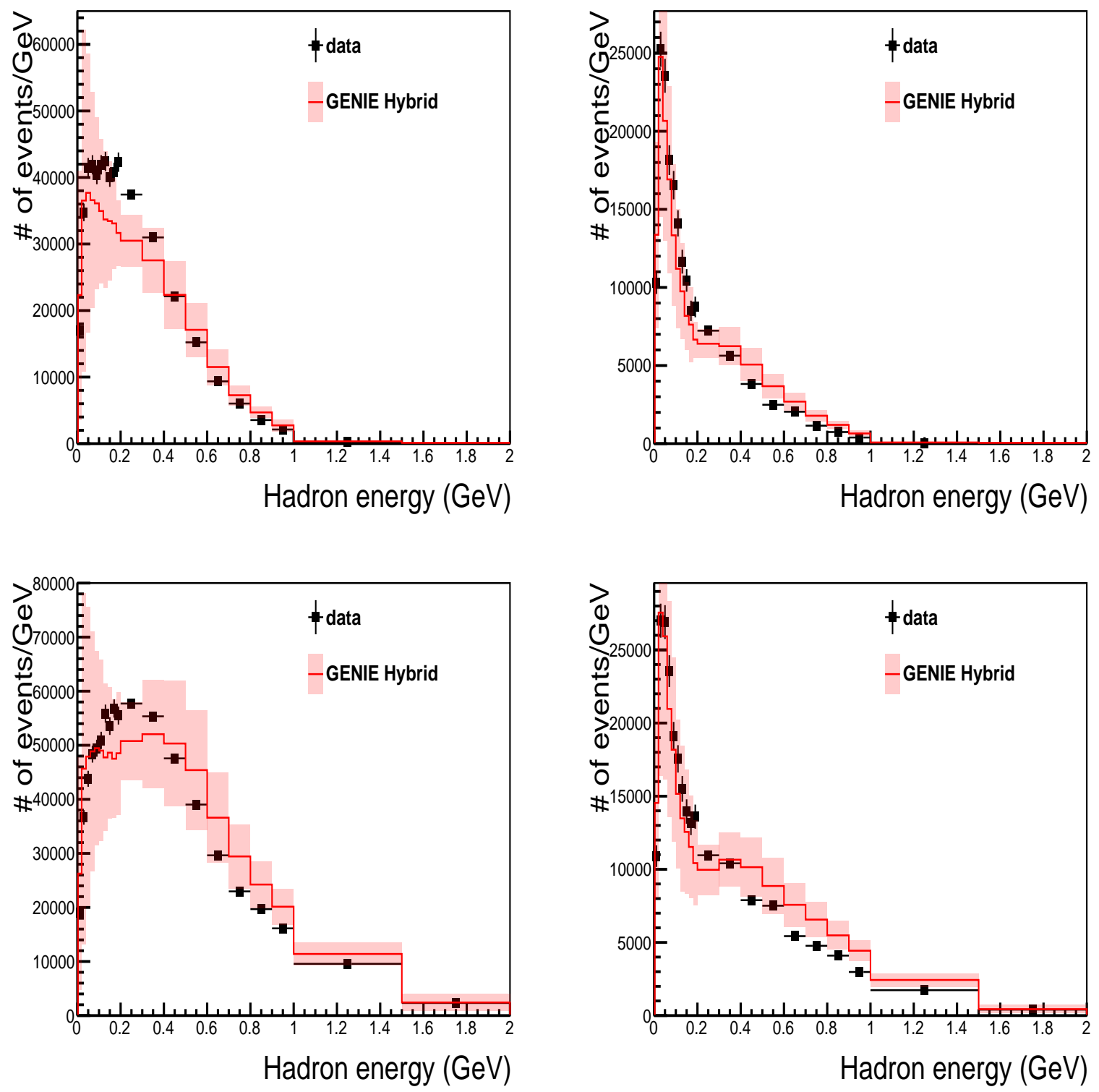

Figure 101: Comparison of data and MC simulation distributions of hadronic energy with $2<$ $E_{\nu}<3 \mathrm{GeV}$ (top) and $3<E_{\nu}<4 \mathrm{GeV}$ (bottom) for FHC neutrinos (left) and RHC antineutrinos(right). The points show data and histograms show GENIE-Hybrid model with low- $\nu$ flux. The error bands includes the statistical error of low- $\nu$ flux reweighting and total systematic error (uncertainty of external normalization not included). Plots are area normalized. 

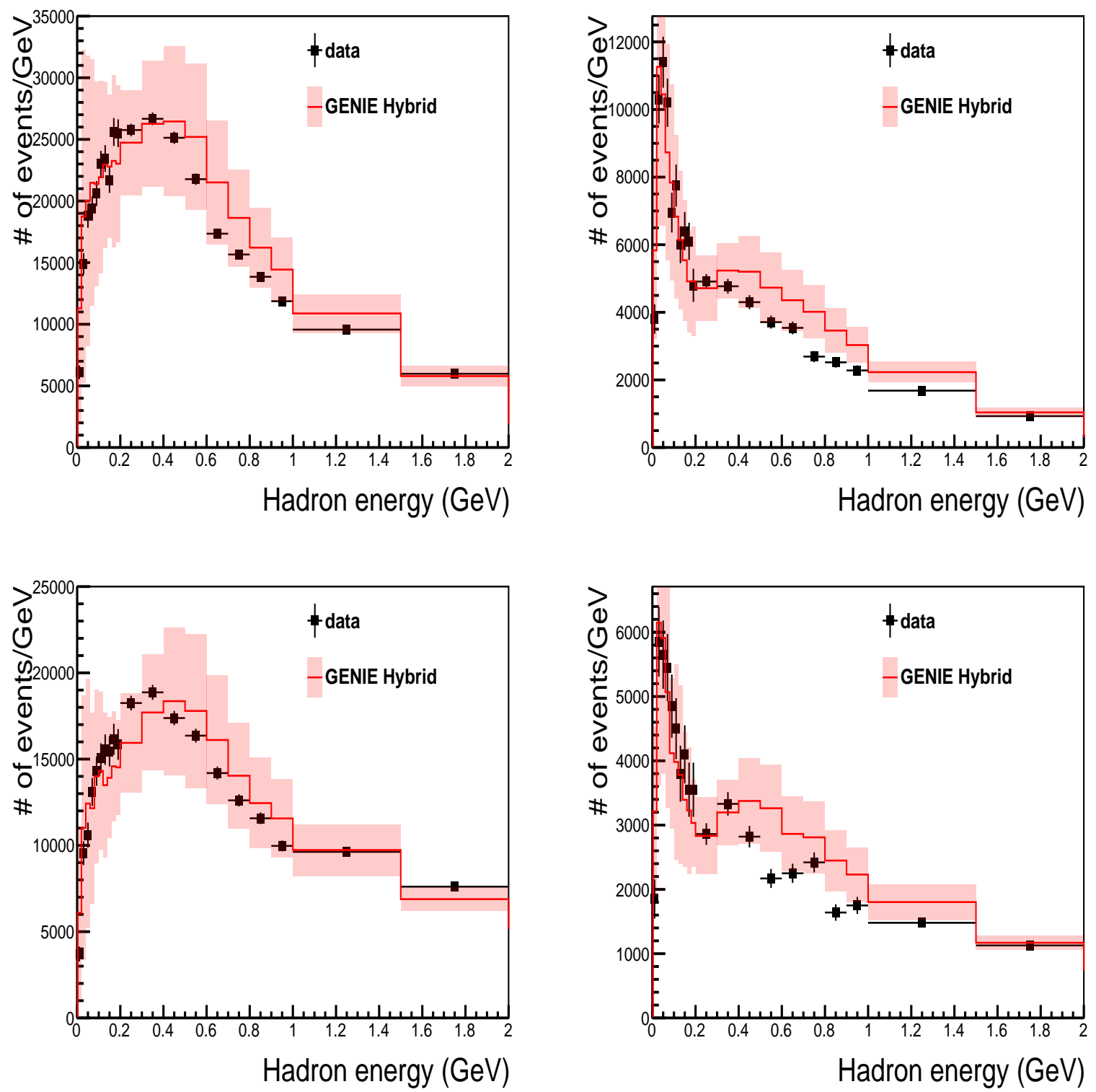

Figure 102: Comparison of data and MC simulation distributions of hadronic energy with $4<$ $E_{\nu}<5 \mathrm{GeV}$ (top) and $5<E_{\nu}<7 \mathrm{GeV}$ (bottom) for FHC neutrinos (left) and RHC antineutrinos(right). The points show data and histograms show GENIE-Hybrid model with low- $\nu$ flux. The error bands includes the statistical error of low- $\nu$ flux reweighting and total systematic error (uncertainty of external normalization not included). Plots are area normalized. 

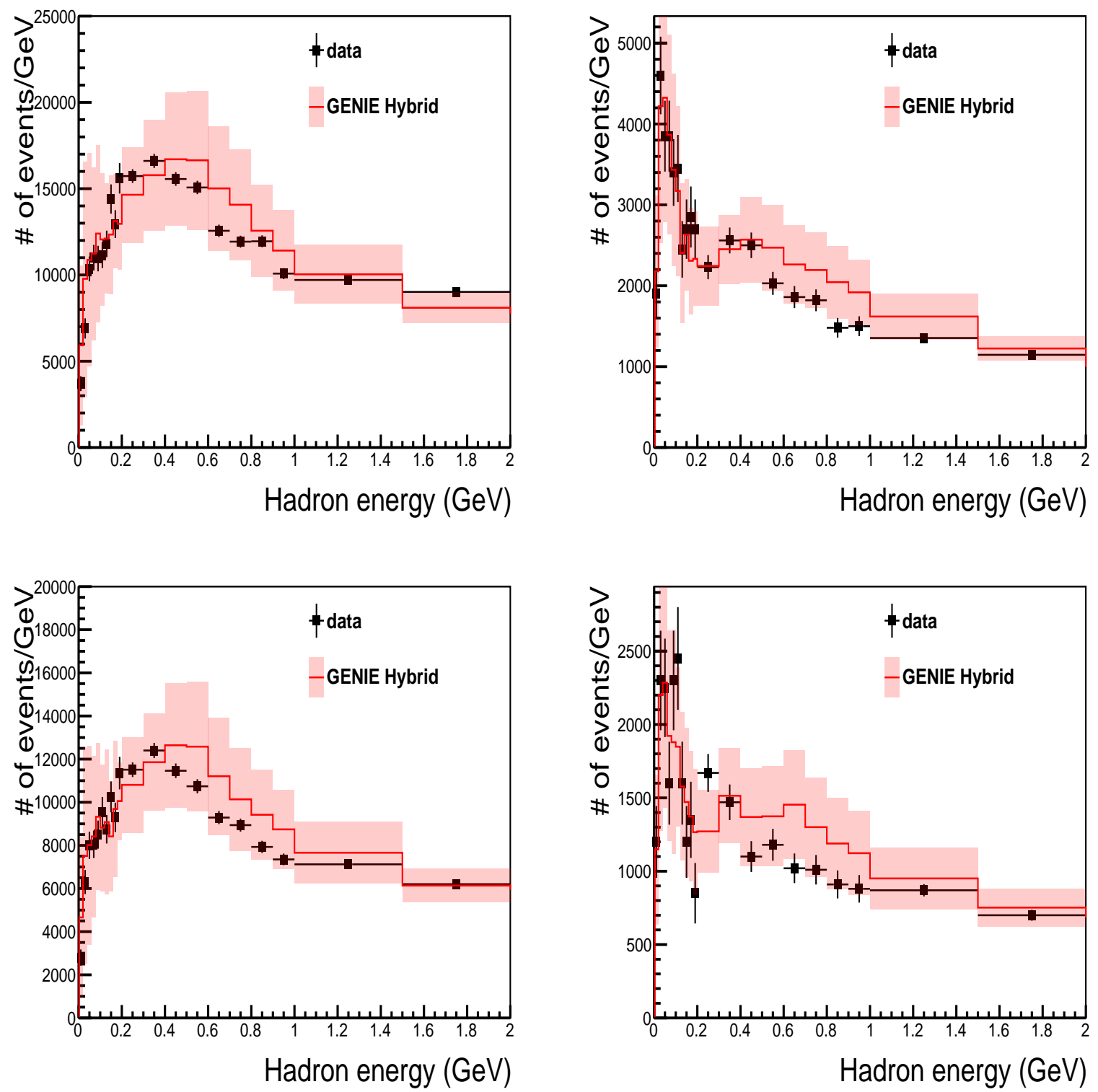

Figure 103: Comparison of data and MC simulation distributions of hadronic energy with $7<E_{\nu}<12 \mathrm{GeV}$ (top) and $12<E_{\nu}<22 \mathrm{GeV}$ (bottom) for FHC neutrinos (left) and RHC antineutrinos(right). The points show data and histograms show GENIE-Hybrid model with low- $\nu$ flux. The error bands includes the statistical error of low- $\nu$ flux reweighting and total systematic error (uncertainty of external normalization not included). Plots are area normalized. 

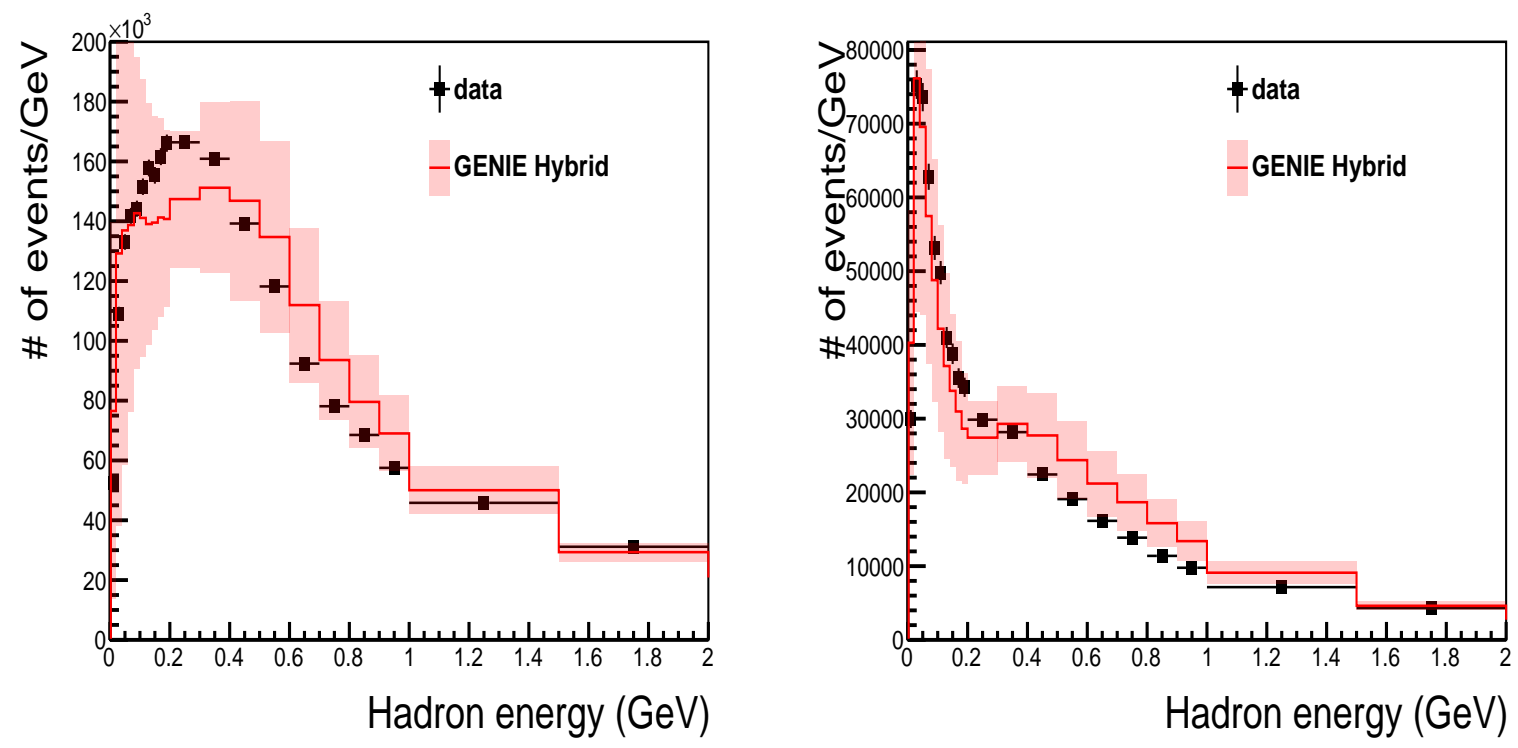

Figure 104: Comparison of data and MC simulation distributions of hadronic energy with $2<$ $E_{\nu}<22 \mathrm{GeV}$ for FHC neutrinos (left) and RHC antineutrinos(right). The points show data and histograms show GENIE-Hybrid model with low- $\nu$ flux. The error bands includes the statistical error of low- $\nu$ flux reweighting and total systematic error (uncertainty of external normalization not included). Plots are area normalized. 


\subsection{CROSS CHECK AND ADDITIONAL STUDIES}

\subsubsection{NuWro-based results}

Since NuWro does not include quark mixing, we apply $G_{c o r r}$ from GENIE-Hybrid model to correct the NuWro extracted results. A comparison of NuWro-based and GENIE Hybrid neutrino cross section is shown in Fig. 89. The first three neutrino energy bins show large differences between the two models. This is mainly due to the difference in kinematics correction $A_{C C}^{K I N}$ (discussed in Sec. 6.3.2). The two results agree at high energy since we normalize each to the same external world data. Fig. 90 shows a comparison for antineutrino cross section and Fig. 91 shows a comparison for the ratio, $r$. For antineutrino, the difference at high energy is due to the different DIS PDF library used by two models, which results in different model-dependent corrections. This gives both neutrino and antineutrino unnormalized cross sections about $1 \%$ difference, which propagates into the normalization constants.

\subsubsection{Extract cross sections with low- $\nu$ flux reweighted}

A comparison of re-extract cross sections with low- $\nu$ flux reweight and nominal results are shown in Fig 105 for neutrino and antineutrino cross sections. The extraction of cross section and flux converges after applying the flux weighted, which shows that this method of extracting cross section is independent of input simulated flux. 

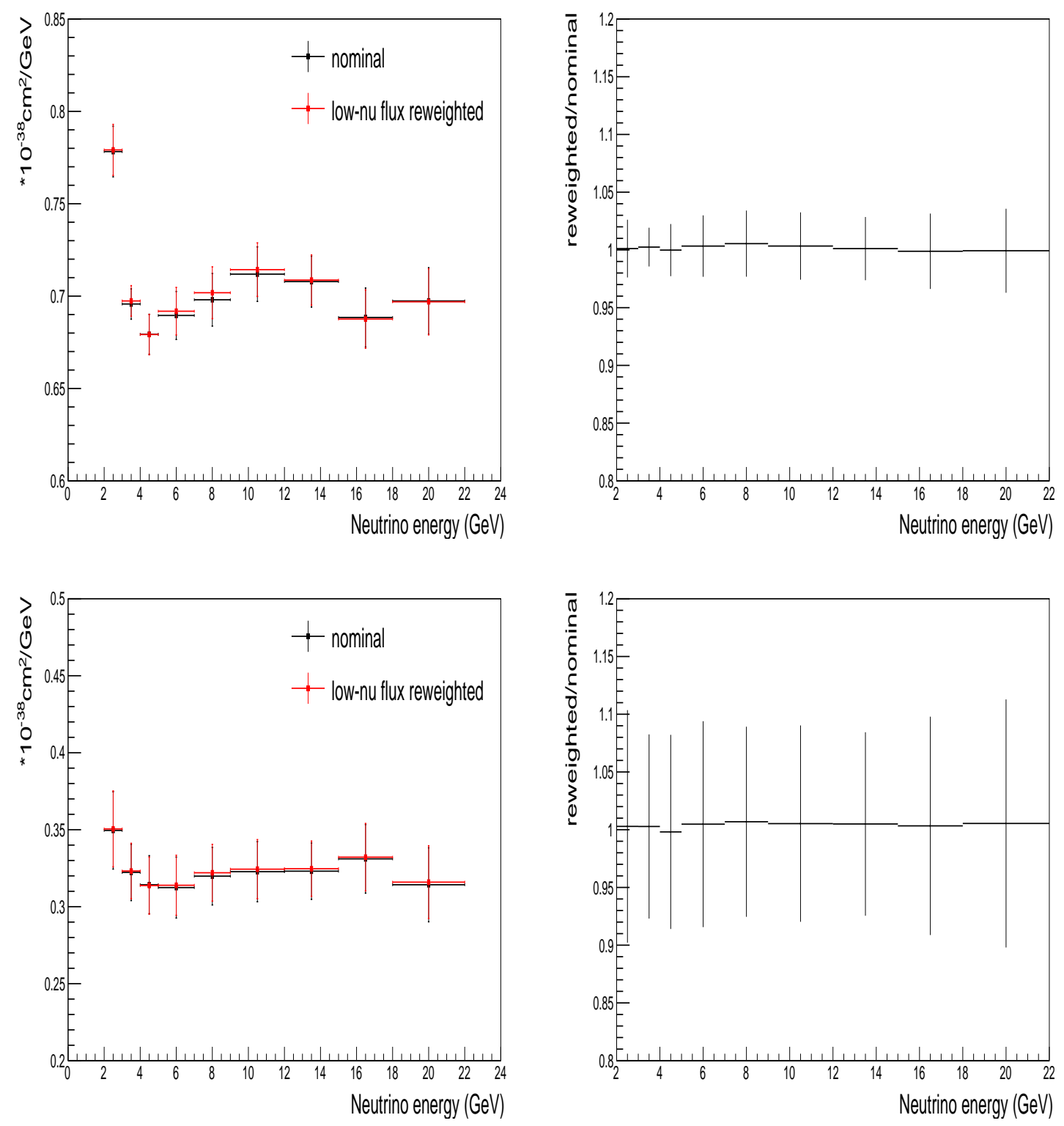

Figure 105: Measured neutrino (top) and antineutrino (bottom) cross sections with low- $\nu$ flux reweighted, comparing with the nominal on the left, the ratio of reweighted to nominal neutrino cross section on the right. Error bars are statistical error only. 


\subsubsection{Extract cross sections in opposite modes}

We extract neutrino cross section from RHC sample and antineutrino cross section from FHC sample, then compared with nominal results. The comparison with nominal results are shown in Fig 106 for neutrino and antineutrino cross sections. The measured quantities overlap above 7 $\mathrm{GeV}$, but show discrepancy below $7 \mathrm{GeV}$. The differences of measured quantities at low energy are likely due to the large wrong-sign contamination in the opposite modes, as shown in Fig. 107. The wrong-sign contamination for FHC antineutrino (RHC antineutrino) is as large as 30\% (50\%) at the lowest neutrino energy bin.

\subsubsection{Extract cross sections in different regions of detector}

We study the MINOS ND geometry effect by extracting cross section in different regions of MINOS ND separated by where the muon track enters it. We divide MINOS ND into left and right regions based on whether a muon enters MINOS ND on the left or right of beam center, (264 mm, $-241 \mathrm{~mm}$ ) in MINER $\nu \mathrm{A}$ coordinate. We extract cross sections and the ratio, $r$, from left and right, respectively. The comparison of left and right are shown in Fig 108 for both neutrino and antineutrino cross sections. The cross sections from left is about $2 \%$ larger than those extracted from right. This asymmetry is due to the modeling of the coil hole of MINOS ND, which has has larger effect on tracks with vertices in the left region.

\subsection{SUMMARY}

In this chapter, we have shown the measured results of neutrino and antineutrino cross section and the ratio, $r$. The energy-dependent shapes of cross sections and the ratio are compared with GENIE Hybrid model. Both cross sections are flat above $9 \mathrm{GeV}$, while at lower energy, both show a dip around $5 \mathrm{GeV}$ comparing with the model. The shape of the ratio, $r$, agrees with GENIE Hybrid model within measurement precision.

The uncertainty of neutrino cross section is dominated by systematic uncertainty, while the uncertainty of the antineutrino cross section and the ratio, $r$, are dominated by statistical precision 

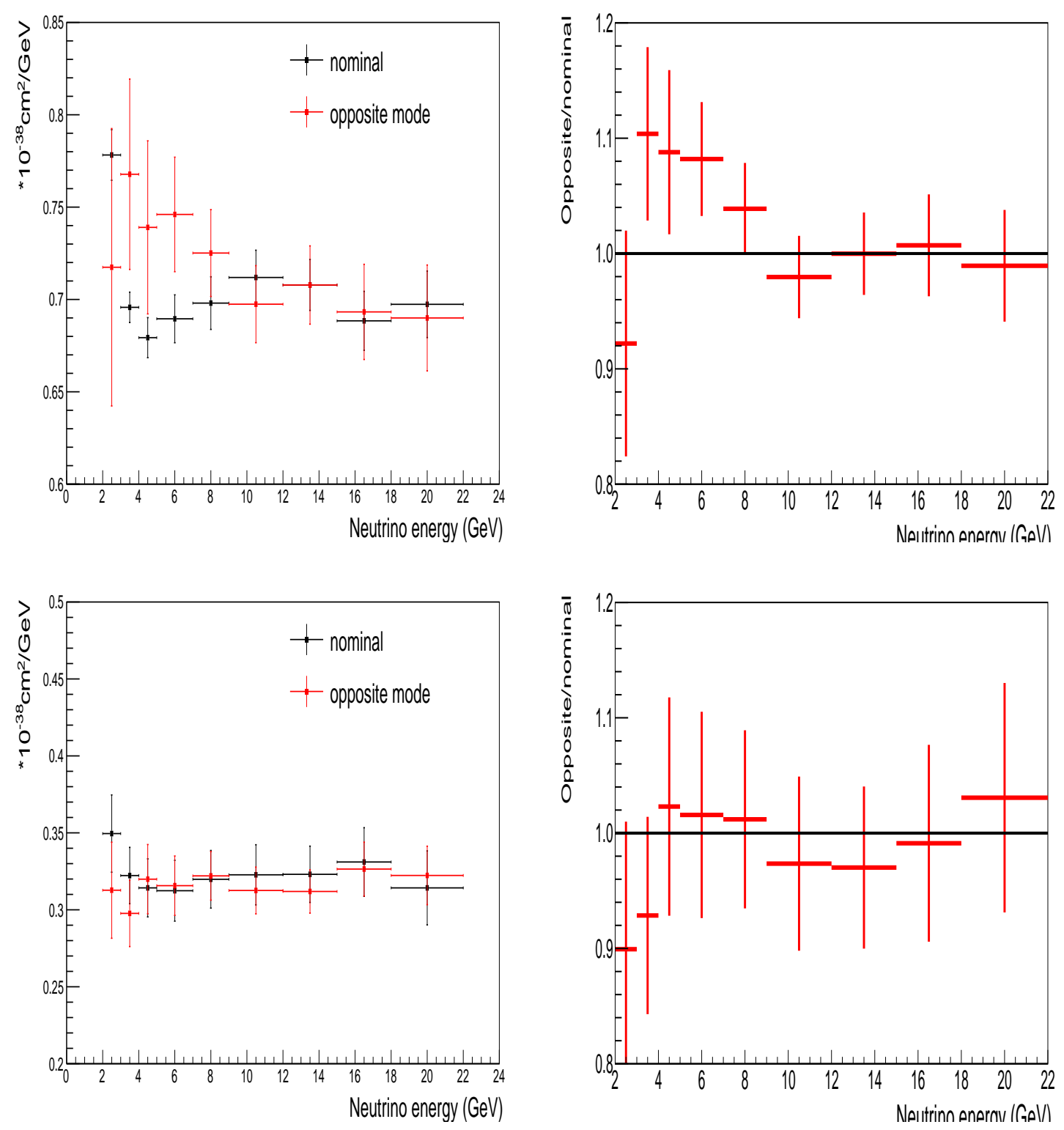

Figure 106: Measured neutrino cross section in RHC mode (top) and antineutrino cross section in FHC mode (bottom), comparing with the nominal on the left, the ratio of opposite mode to nominal neutrino cross section on the right. Error bars are statistical error only.

as shown in Fig. 93 and Fig. 94. Therefore, increasing the size of antineutrino data sample will improve the accuracy of antineutrino cross section and the ratio, $r$. 

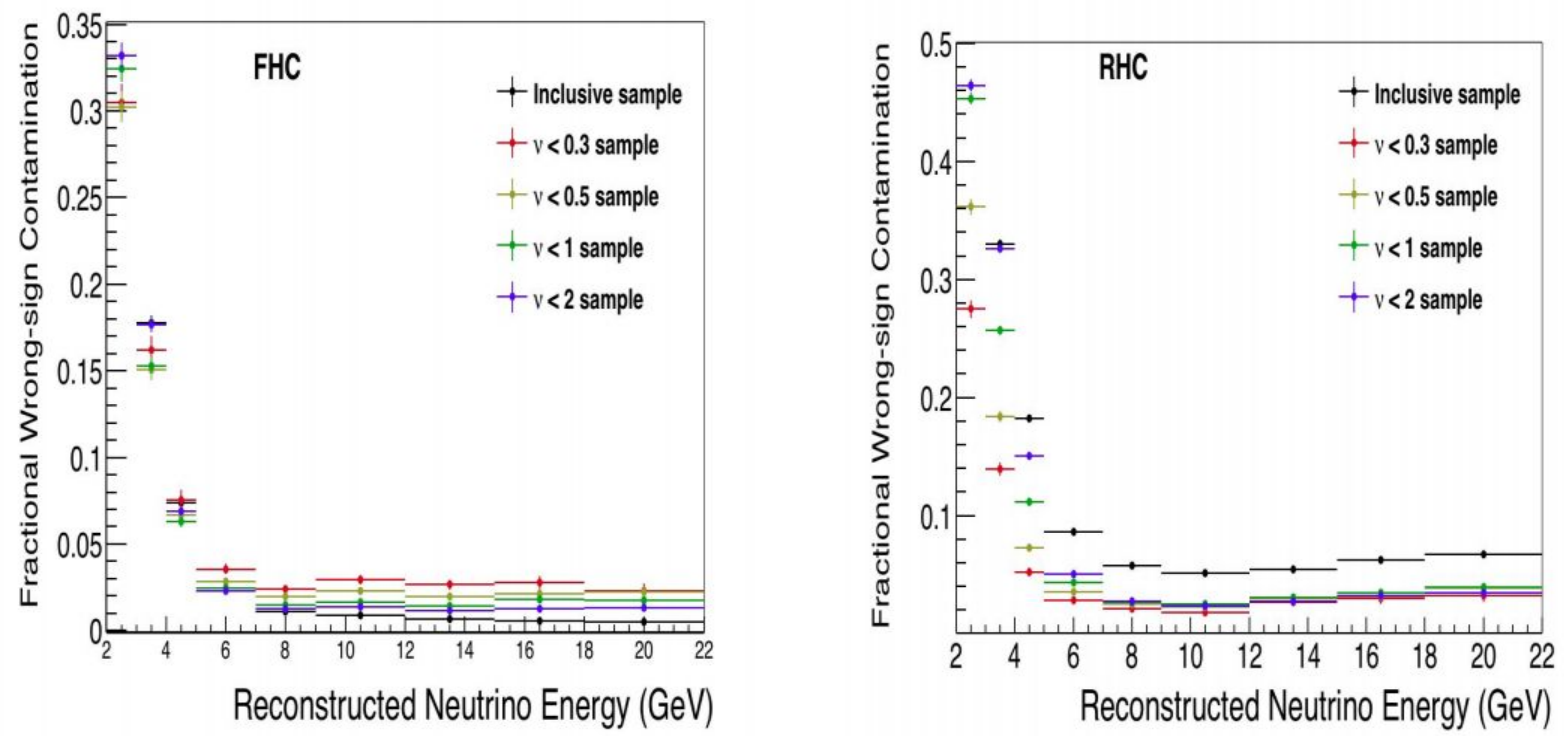

Figure 107: Wrong-sign contamination for FHC antineutrino (left) and RHC neutrino (right) samples.

Cross sections and the ratio using NuWro based model corrections are also shown. The NuWro based results have statistical error only. A comparison of GENIE Hybrid and NuWro based results show that the cross sections agree well above $3 \mathrm{GeV}$, while in 2-3 GeV, NuWro based results are about $15 \%$ below GENIE Hybrid version, which is due to different modeling.

We also measured the neutrino flux and antineutrino flux. Both neutrino and antineutrino fluxes show good agreement with gen2-thin flux, which is the simulated flux modeled with hadron production data. 

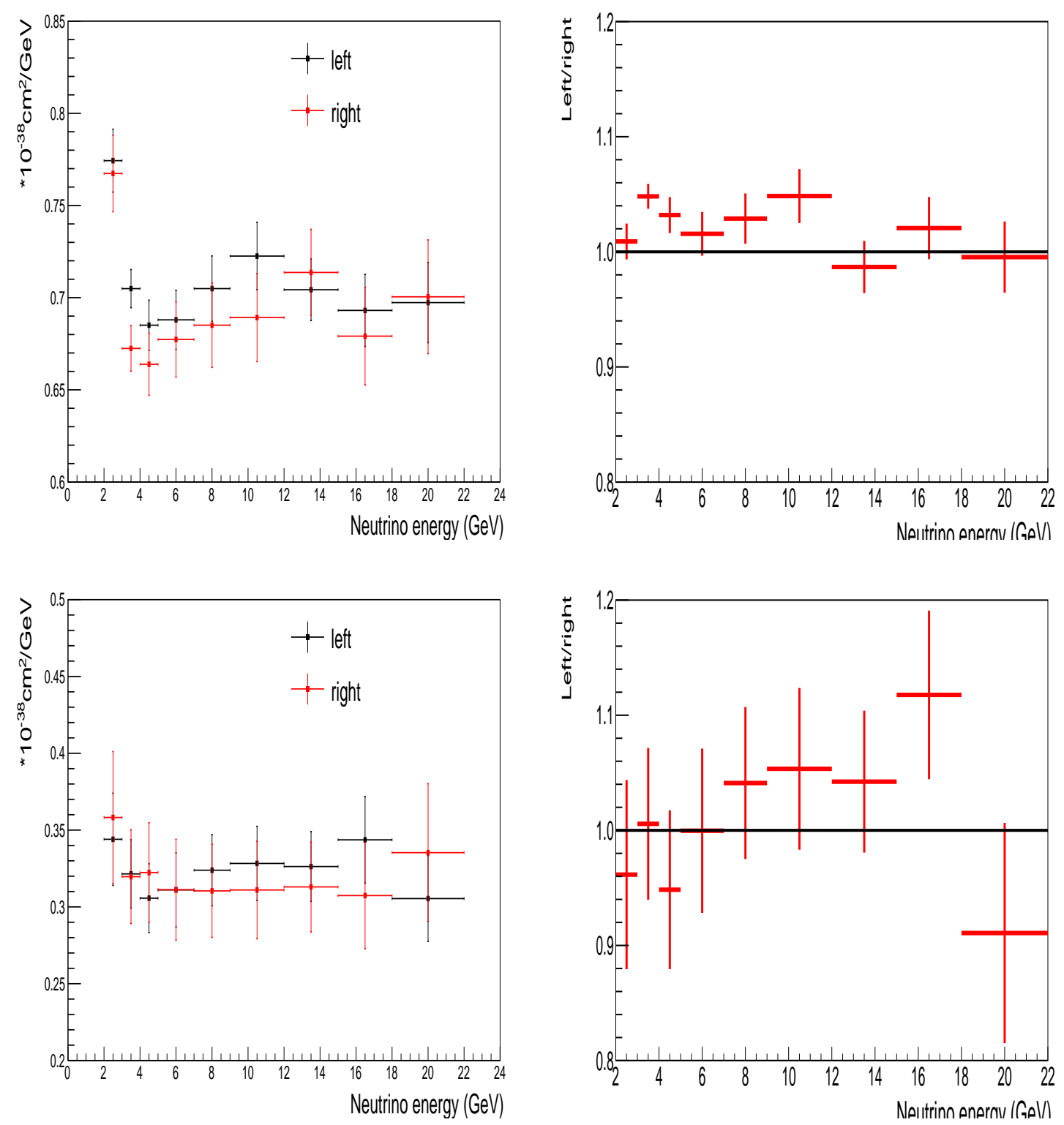

Figure 108: Measured neutrino (top) and antineutrino (bottom) cross sections from left and right of MINOS near detector on the left, ratio of left to right on the right. Error bars are statistical error only. 


\subsection{CONCLUSION}

We have presented the measurements of neutrino and antineutrino charged-current inclusive cross sections and the antineutrino to neutrino cross section ratio in the energy region 2-22 GeV with MINER $\nu \mathrm{A}$ scintillator tracker.

We used low- $\nu$ method with model-dependent terms factorized out to allow comparison of different cross section models. Results were obtained using two models, GENIE Hybrid, as well as using alternative NuWro model corrections.

GENIE Hybrid results are compared with world data, as shown in Fig. 109 and Fig. 110. Our measurement of antineutrino cross section, with an uncertainty of $6.1 \%-10.5 \%$, is the most precise to date. The ratio, $r$, with an uncertainty of $5.0 \%-7.5 \%$, is the most precise measurement below $7 \mathrm{GeV}$ to date. Uncertainty of both antineutrino and ratio measurements can be reduced by increasing the antineutrino data sample size.

We also present the inclusive and low- $\nu$ event rates, which can be used to obtain results with alternative models. This allows the use of MINER $\nu \mathrm{A}$ data with model corrections computed from improved models in future.

In future long baseline neutrino oscillation experiments, which are designed to determine unknown oscillation parameters, measurements of neutrino and antineutrino spectral shapes play an important role. Our cross section measurements can be used to improve precision on these measurements. 


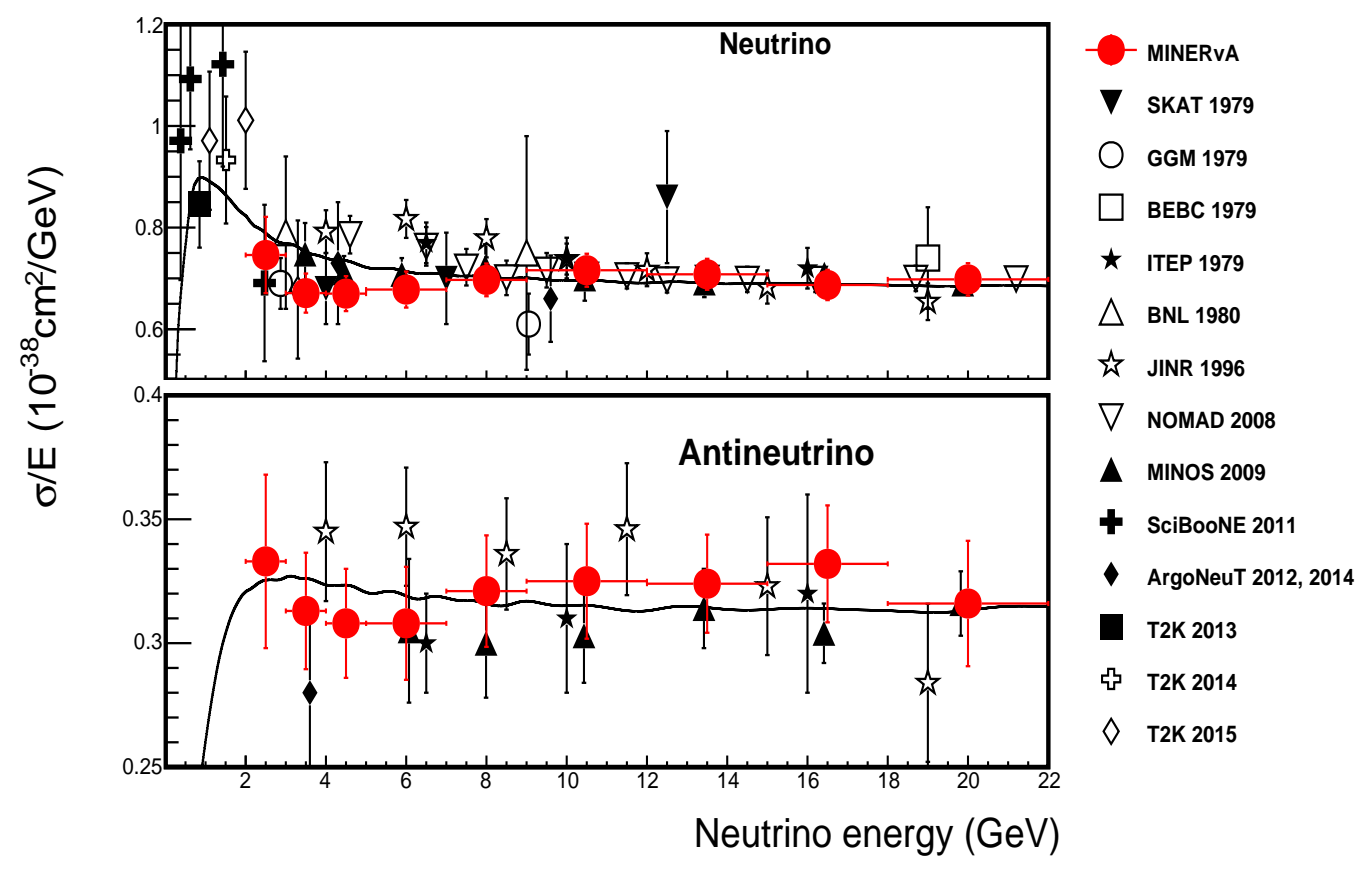

Figure 109: Extracted neutrino and antineutrino cross sections, compared with world data. 


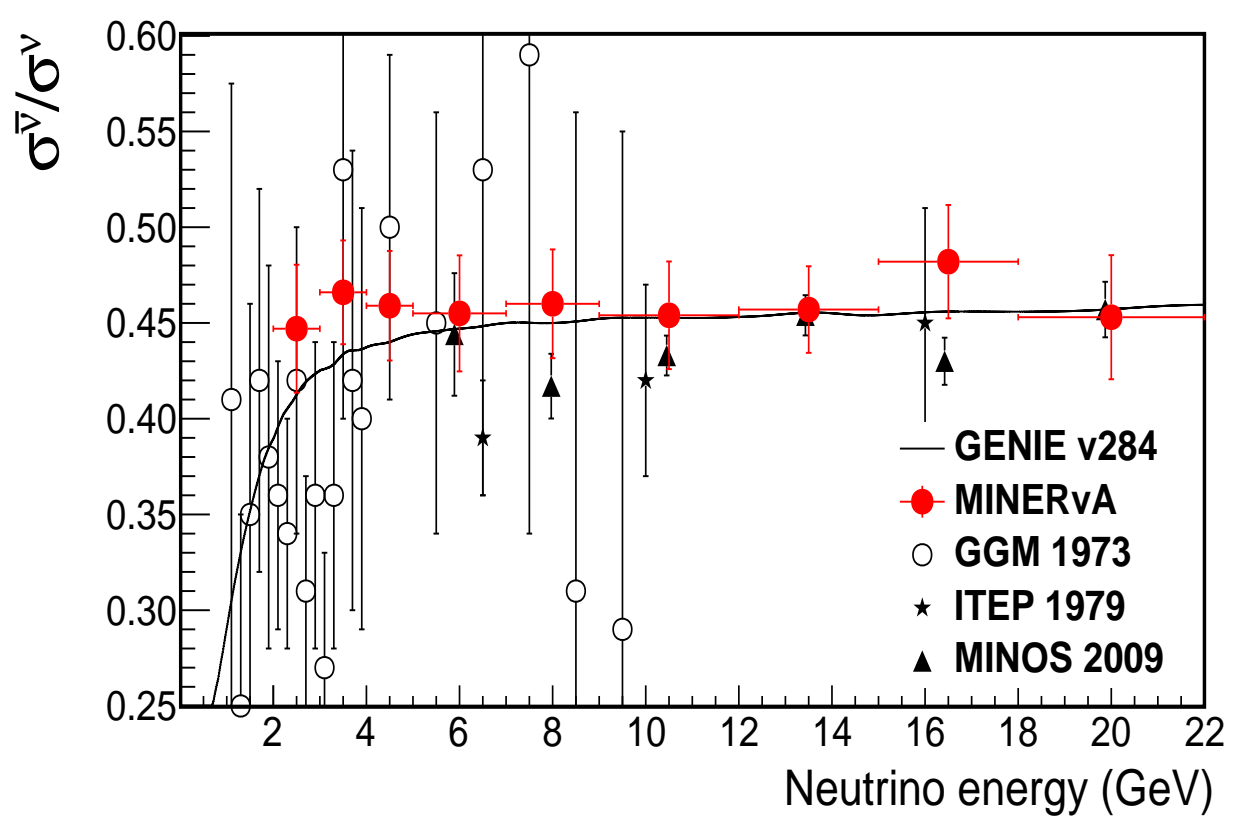

Figure 110: Extracted cross section ratio, $r$, compared with world data. 


\section{APPENDIX A}

\section{CYLINDER TUNING}

For shower cleaning correction in the cylinder region, we want to optimize the distance from vertex $\mathrm{d}$ and radii $r_{i}(i=$ tracker, ecal, hcal $)$, to maximize the true muon energy inside the cylinder and remove it from the recoil system and put back into muon energy.

In this study, we use two MC samples, an inclusive charged-current events sample and a MINOS-matched rock muon sample. We use truth information of inclusive sample to study hadronic energy and muon energy separation. Rock muon sample is also used since there is no hadronic shower and all the non-track energy are known to be muon fuzz.

First, we define $E_{i}^{s h, r_{i}}$ as the sum of energies of all non-muon track clusters found inside subdetector $i$ within radius $r_{i}$, which can be written as

$$
E_{i}^{s h, r_{i}}=\Sigma_{j} E_{i, j}^{s h, r_{i}}
$$

$j$ stands for the cluster id based on truth information, which can be hadron(including proton, pion, neutron and etc.) muon or cross talk.

Three parameters are then defined for each sub-detector, which are muon purity $F_{i}$, hadron completeness $C_{i}$ and muon completeness $T_{i}$.

Muon purity $F_{i}$ is defined as the fraction of true muon energy out of all cluster energy found inside the cylinder,

$$
F_{i}=\frac{E_{i, m u o n}^{s h, r_{i}}}{E_{i}^{s h, r_{i}}},
$$

in which true cross talk is removed from the denominator. 
Hadron completeness $C_{i}$ is defined as the fraction of true hadron energy found outside a cylinder out of all true hadron energy found in the whole inner detector.

$$
C_{i}=\frac{E_{i, \text { hadron }}^{\text {sh,R }}-E_{i, \text { hadron }}^{\text {sh, } r_{i}}}{E_{i, \text { hadron }}^{\text {sh,R }}}
$$

$R=1000 \mathrm{~mm}$ stand for the whole inner detector.

Muon completeness $T_{i}$ is defined as the fraction of muon energy inside the cylinder to the whole inner detector

$$
T_{i}=\frac{E_{i, \text { muon }}^{s h, r_{i}}}{E_{i, \text { muon }}^{\text {sh,R }}}
$$

$F_{i}$ and $C_{i}$ are obtained from inclusive MC sample, while $T_{i}$ is from rock muon MC sample. We vary the radii of cylinder to collect as much true muon fuzz energy as possible, while at the same time, we also want to minimize the true hadron energy inside the cylinder.

Figure 111 shows the purity and completeness for tracker region for distance $d=100 \mathrm{~m}$ and $\mathrm{d}=1000 \mathrm{~mm}$, with $r_{\text {tracker }}$ varying from $20 \mathrm{~mm}$ to $200 \mathrm{~mm}$, for inclusive samples and low- $\nu$ samples. For the same distance d, as radius increases, purity drops a few percents for all sample, and larger $\mathrm{d}$ always has a higher purity. Hadron completeness also drops as radius increases, which means more true hadron energy is collected inside the cylinder.

To be consistent with MINERvA's CCQE analysis, we choose distance from vertex d=300mm. Figure 112 shows the purity and completeness for $d=300 \mathrm{~mm}$. We choose radius to be $80 \mathrm{~mm}$ in the tracker, so that the purities for cross section sample and flux samples are always above $60 \%$.

A similar study is performed for Ecal and Hcal regions. Figure 113 shows the purity and completeness for Ecal and Hcal. We choose cylinder radius in Ecal and Hcal to be $100 \mathrm{~mm}$, so the purity for both Ecal and Hcal is above $80 \%$. An energy correction, $E_{i n}^{s h}$, is defined from inside this cylinder as

$$
E_{i n}^{s h}=\Sigma_{i} E_{i}^{s h, r_{i}} F_{i}
$$

in which $E_{i}^{f u z z}$ is the non-muon track energy found inside the cylinder region in subdetector i. 

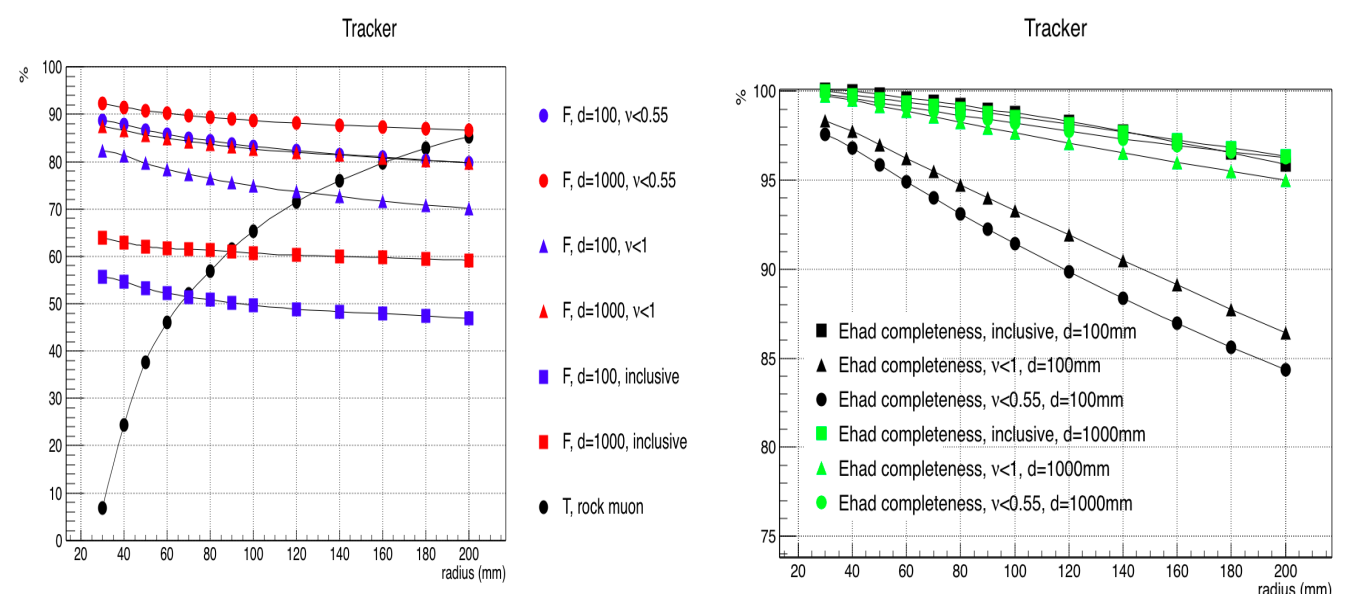

Figure 111: Muon purity and hadron completeness for different radii in the tracker region.

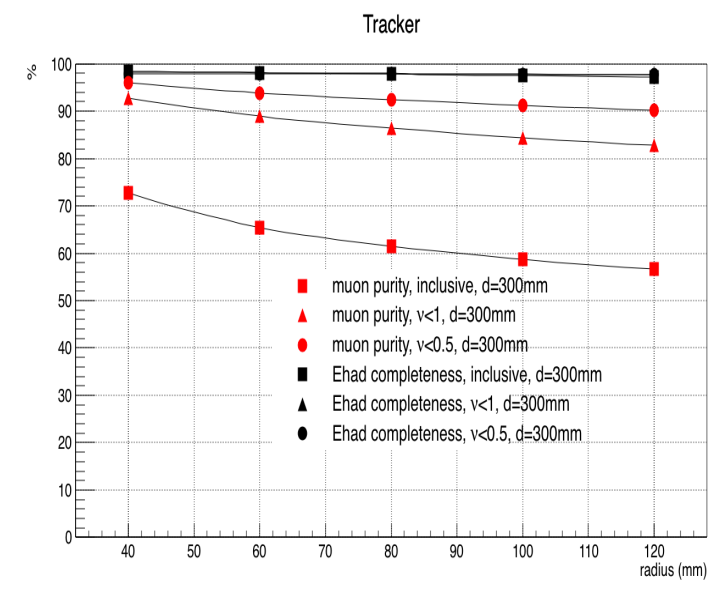

Figure 112: Muon purity and hadron completeness in tracker with $d=300 \mathrm{~mm}$. 


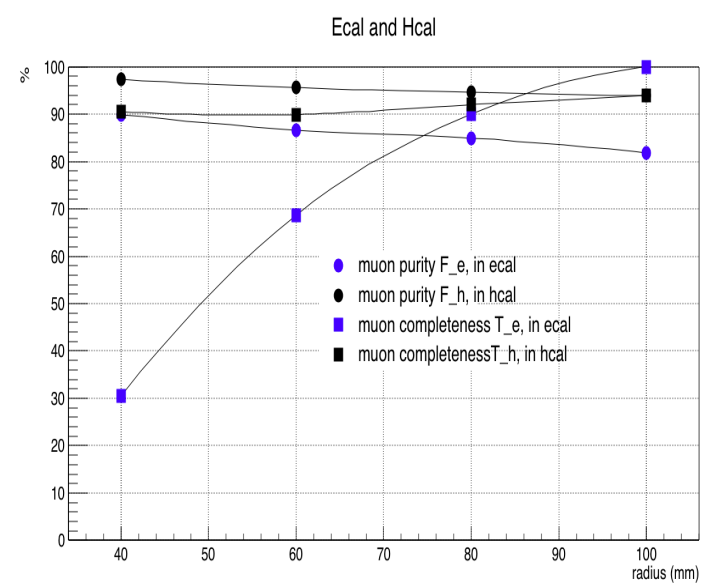

Figure 113: Muon purity and muon completeness for different radii in Ecal and Hcal. 


\section{APPENDIX B}

\section{COVARIANCE MATRICES OF MEASURED QUANTITIES}

In this appendix, we present the joint covariance matrix and correlation matrix of neutrino and antineutrino cross sections, covariance matrix for the ratio, $r$, and $\chi^{2}$ calculation using the covariance matrix.

\section{B.1 COVARIANCE MATRIX}

\section{B.1.1 Definition}

The covariance matrix for neutrino and antineutrino cross section is an $18 \times 18$ matrix, of which the 1-9 rows and columns are for neutrino cross section and the 10-18 rows and columns are for antineutrino cross sections. It is a linear sum of covariance matrix of each error source, excluding the overall external normalization piece. For the element of covariance matrix is defined as

$$
\operatorname{cov}_{i, j}=\frac{1}{N} \sum_{k=1}^{N}\left(x_{k i}-\bar{x}_{i}\right)\left(x_{k j}-\bar{x}_{j}\right),
$$

where $i, j$ are from 1 to 18 , index 1-9 are for 9 bins of neutrino cross section in the energy range 2$22 \mathrm{GeV}$ and index 10-18 are for 9 bins of antineutrino cross section in the energy range 2-22 GeV. For muon energy scale and hadron energy scale, $N=100, k$ stands for the $k t h$ universe. $\bar{x}_{i}$ is the mean value in bin $i$ average over 100 universes and $x_{k i}$ is the value of bin $i$ in $k$ th universe. For 
GENIE FSI and cross section model uncertainties, the same definition applies with $N=2$ and $\bar{x}_{i}$ is the mean value in bin i average of 2 universes.

For normalization, muon fuzz, RPA and $2 \mathrm{p} 2 \mathrm{~h}$, which are evaluated in 1 universe (turning on and off), the definition of covariance matrix is

$$
\operatorname{cov}_{i, j}=\left(x_{i}-x_{i}^{c v}\right)\left(x_{j}-x_{j}^{c v}\right)
$$

where $i$ and $j$ stand for ith or jth bin, $x_{i}$ is the shifted value in bin $i$ and $x_{i}^{c v}$ is the central value in bin $i$. This definition also applies for statistical error, but all off-diagonal elements are required to be zero, since there is no unfolding in this analysis, the neighboring bins are uncorrelated.

\section{B.1.2 Covariance Matrix}

Two versions of covariance matrix ${ }^{1}$ are shown for neutrino and antineutrino cross sections,

- Covariance matrix corresponding to total error (including both statistical and systematic errors, except for external normalization uncertainty), which is shown in Fig. 114 and Tab. 52.

- Covariance matrix corresponding to systematic error only, which is shown Tab. 53.

The contribution from statistical error is shown in Fig. 115 in Sec. B.1.3. The covariance matrix corresponding to total error for the ratio, $r$, is shown in Tab. 54.

\section{B.1.3 Components of Covariance Matrix}

In this section, we show components which have larger contribution to covariance matrix of cross sections and the ratio, $r$.

Statistical error dominates the total error for antineutrino cross section and the ratio measurements. Fig. 115 shows the covariance matrix of statistical error for neutrino and antineutrino cross sections. Fig. 116 shows the covariance matrix of statistical error for the ratio, $r$.

For neutrino and antineutrino cross sections, three largest components of systematic error coming from cross section model uncertainty, FSI uncertainty and hadron energy scale (other than external normalization uncertainty), which are shown in Fig. 117, Fig. 118 and Fig. 119, respectively.

\footnotetext{
${ }^{1}$ Since covariance and correlation matrices are symmetric, we only show the upper right elements of matrices in tables.
} 


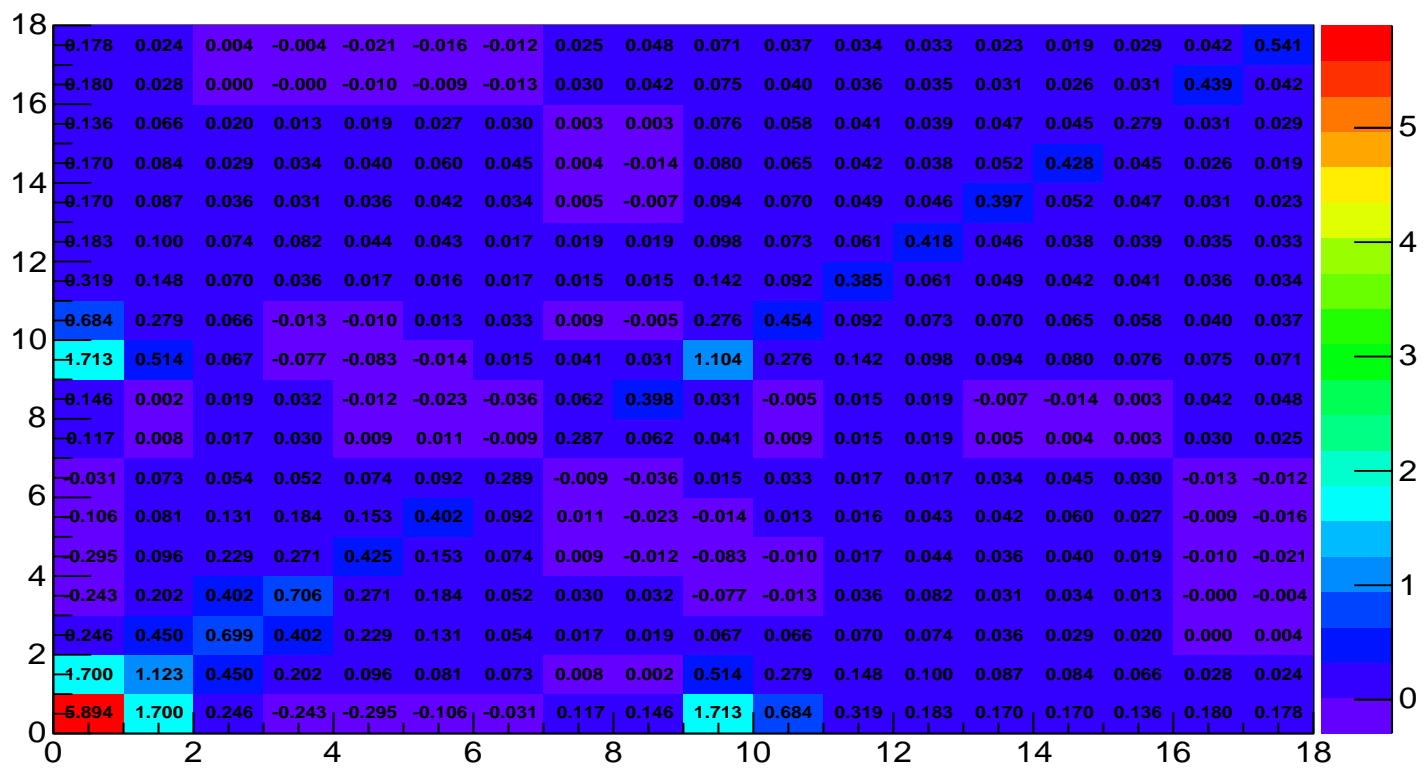

Figure 114: Covariance matrix for the extracted neutrino cross section in the FHC and antineutrino cross section in the RHC beam mode. It includes statistical error and systematic error. The bin boundaries are in units of GeV. The covariance elements are in units of $\left(10^{-38} \mathrm{~cm}^{2} / \mathrm{GeV}\right)^{2}$ and scaled by a factor of 1000. Index 1-9 are for neutrino cross section and 10-18 are for antineutrino cross section. Numbers are also shown in Tab. 52.

For the cross section ratio $r$, the systematic uncertainties between neutrino and antineutrino cross section cancel, and the three largest components of systematic error coming from cross section model uncertainty, FSI uncertainty and muon energy scale, which are shown in Fig. 120, Fig. 121, Fig. 122. 


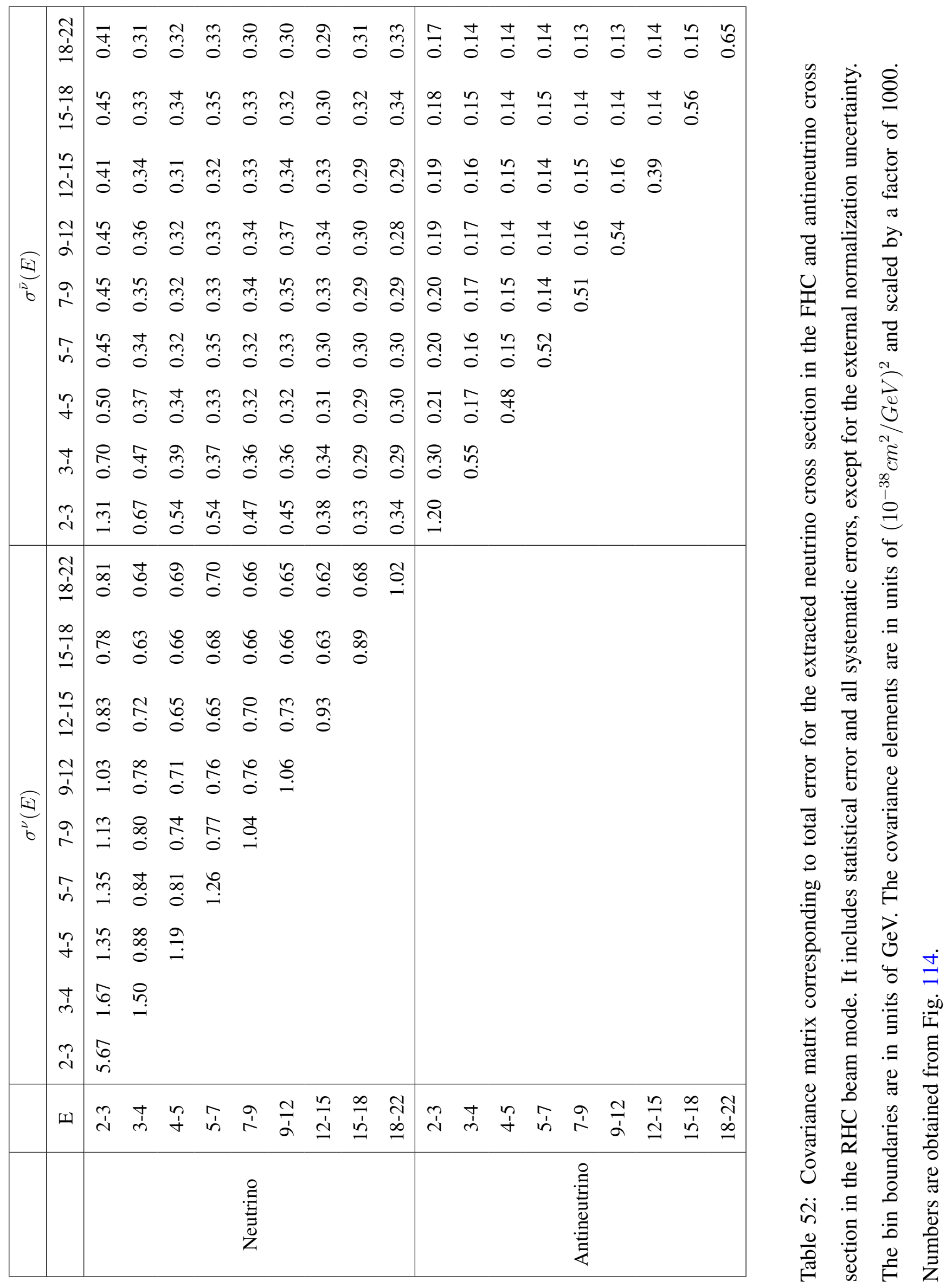




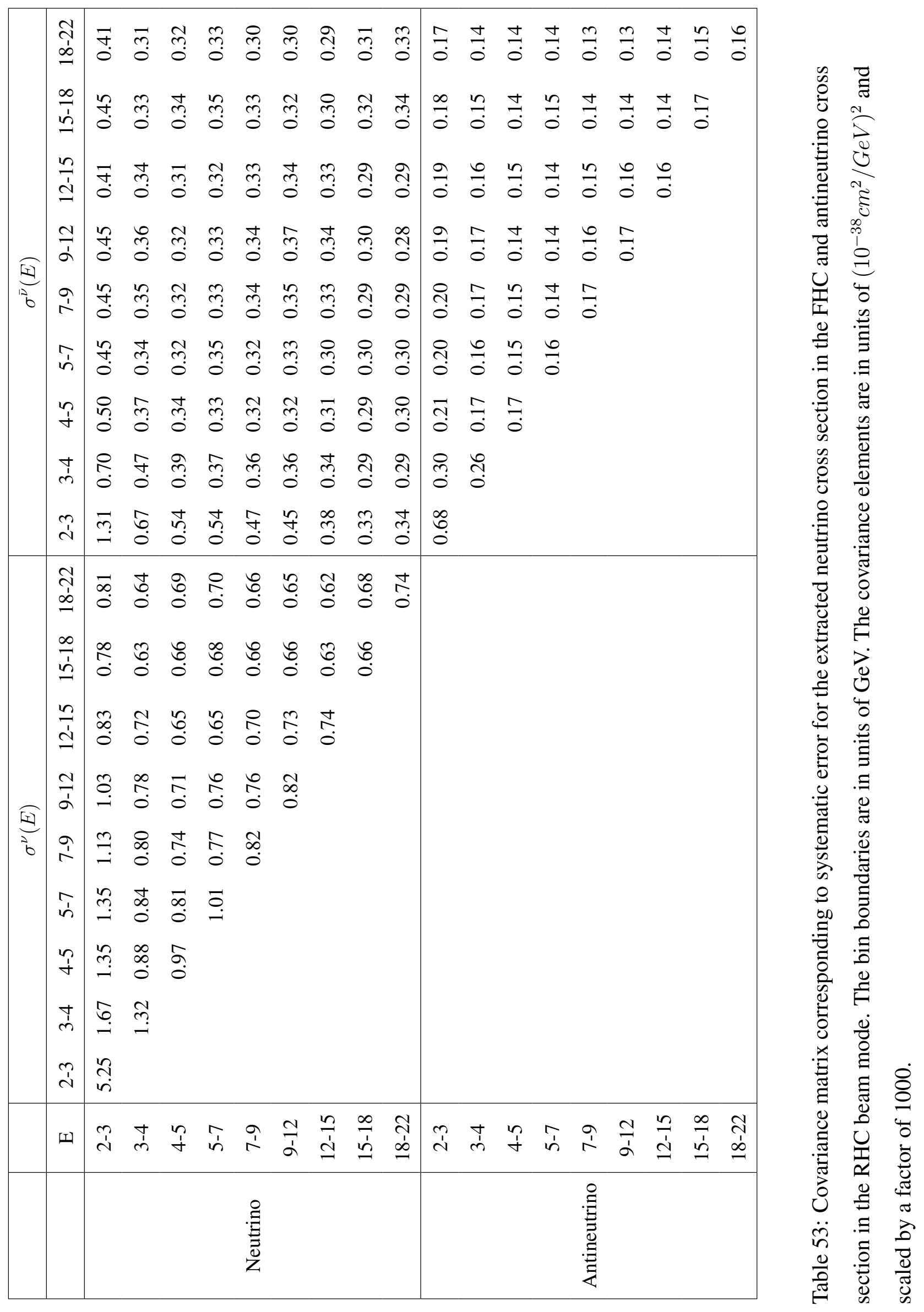




\begin{tabular}{|c|ccccccccc|}
\hline & $2-3$ & $3-4$ & $4-5$ & $5-7$ & $7-9$ & $9-12$ & $12-15$ & $15-18$ & $18-22$ \\
\hline $2-3$ & 1.081 & 0.092 & 0.025 & 0.005 & -0.022 & -0.017 & -0.030 & -0.018 & -0.010 \\
$3-4$ & & 0.729 & 0.131 & 0.116 & 0.063 & 0.043 & 0.012 & 0.016 & 0.020 \\
$4-5$ & & & 0.829 & 0.161 & 0.110 & 0.070 & 0.034 & 0.032 & 0.025 \\
$5-7$ & & & & 0.927 & 0.113 & 0.087 & 0.048 & 0.046 & 0.039 \\
$7-9$ & & & & & 0.800 & 0.062 & 0.034 & 0.034 & 0.030 \\
$9-12$ & & & & & & 0.789 & 0.031 & 0.032 & 0.028 \\
$12-15$ & & & & & & & 0.507 & 0.032 & 0.015 \\
$15-18$ & & & & & & & & 0.872 & 0.017 \\
$18-22$ & & & & & & & & & \\
\hline
\end{tabular}

Table 54: Covariance matrix of extracted cross section ratio, $r$, scaled by 1000. It includes statistical error and all systematic errors. 


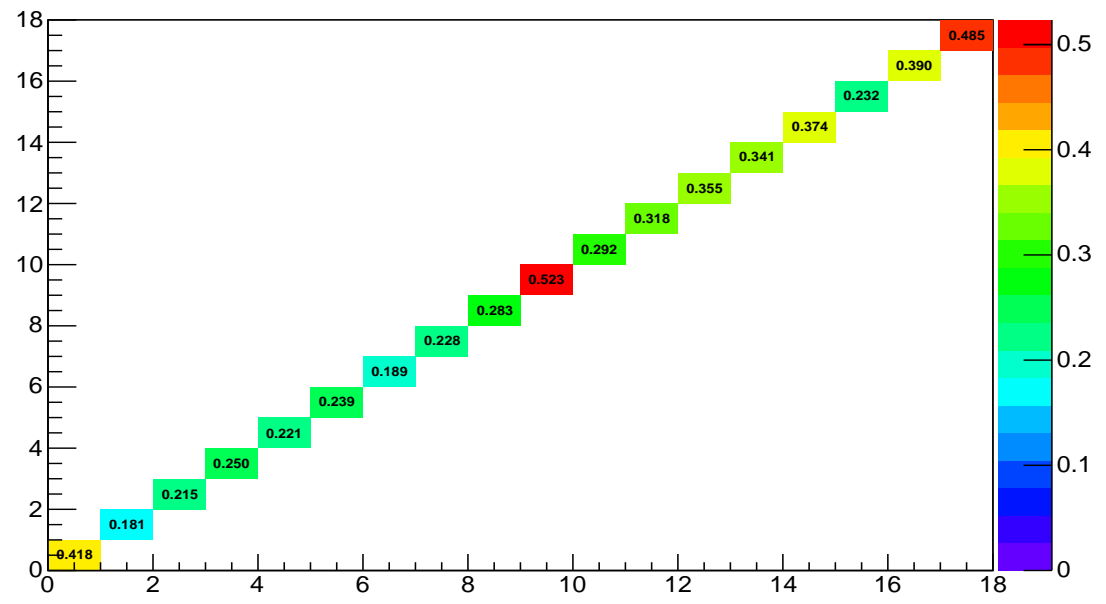

Figure 115: Statistical error component of Covariance matrix for the extracted neutrino cross section in the FHC and antineutrino cross section in the RHC beam mode. The bin boundaries are in units of GeV. The covariance elements are in units of $\left(10^{-38} \mathrm{~cm}^{2} / \mathrm{GeV}\right)^{2}$ and scaled by a factor of 1000. Index 1-9 are for neutrino cross section and 10-18 are for antineutrino cross section. 


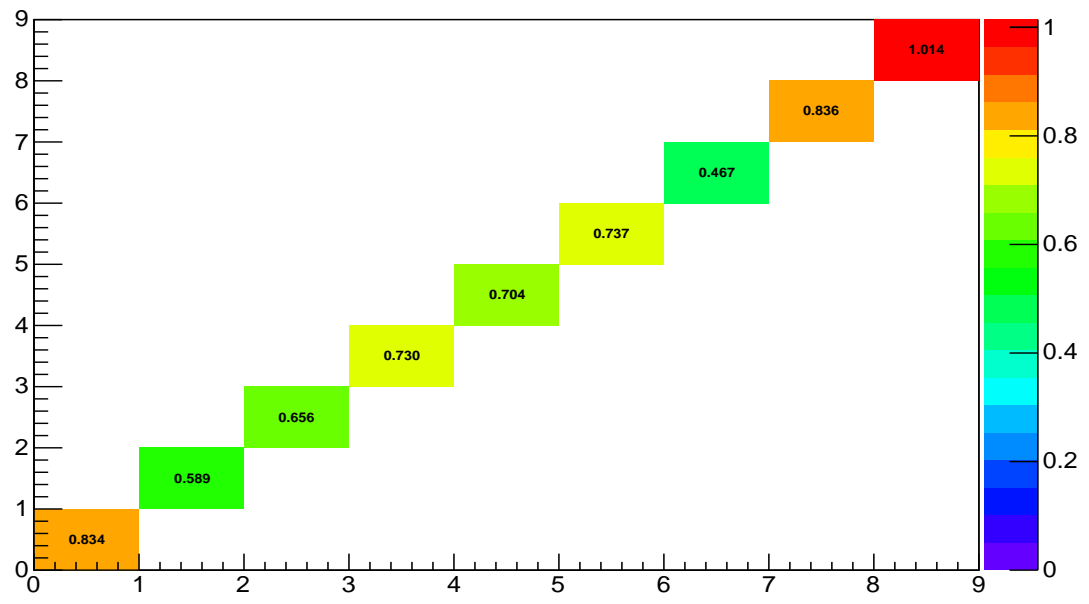

Figure 116: Components coming from statistical error for the ratio. The bin boundaries are in units of $\mathrm{GeV}$. The covariance elements are scaled by a factor of 1000 .

\section{B.2 CORRELATION MATRIX}

Correlation matrix is defined as

$$
\operatorname{corr}_{i, j}=\frac{\operatorname{cov}_{i, j}}{\left(\operatorname{cov}_{i, i} \times \operatorname{cov}_{j, j}\right)^{1 / 2}},
$$

in which $\operatorname{cov}_{i, j}$ is the covariance matrix defined in Eq. B.1. Two versions of correlation matrix are shown:

- Correlation matrix corresponding to total error (including both statistical and systematic errors), which is derived from covariance matrix in Tab. 52, is shown in Tab. 55.

- Correlation matrix corresponding to systematic error only, which is derived from covariance matrix in Tab. 53, is shown in Tab. 56. 


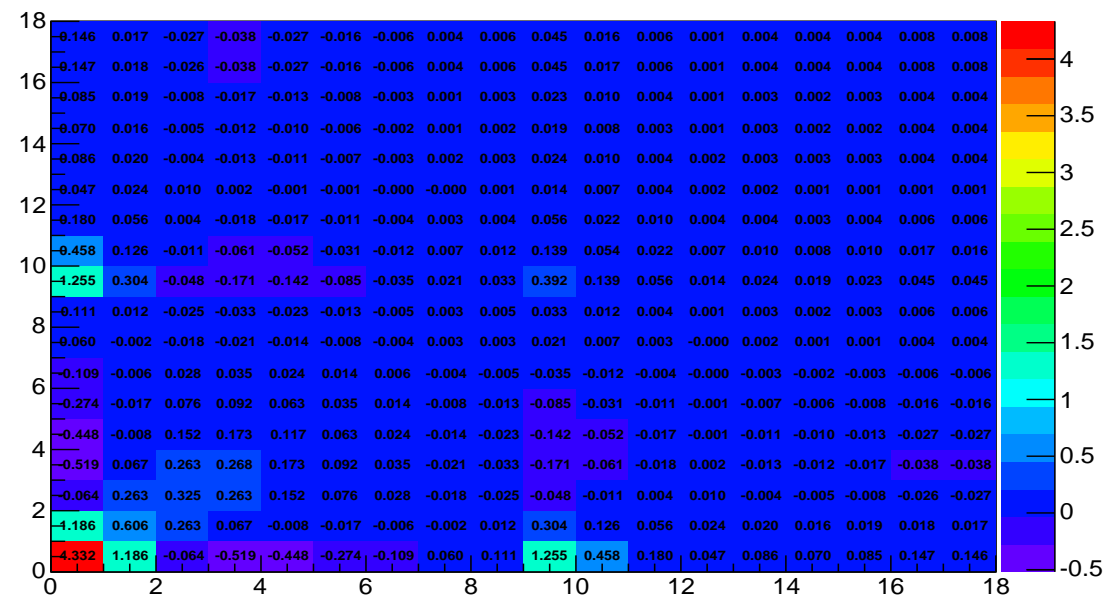

Figure 117: GENIE cross section model uncertainty component of Covariance matrix for the extracted neutrino cross section in the FHC and antineutrino cross section in the RHC beam mode. The bin boundaries are in units of GeV. The covariance elements are in units of $\left(10^{-38} \mathrm{~cm}^{2} / \mathrm{GeV}\right)^{2}$ and scaled by a factor of 1000. Index 1-9 are for neutrino cross section and 10-18 are for antineutrino cross section. 


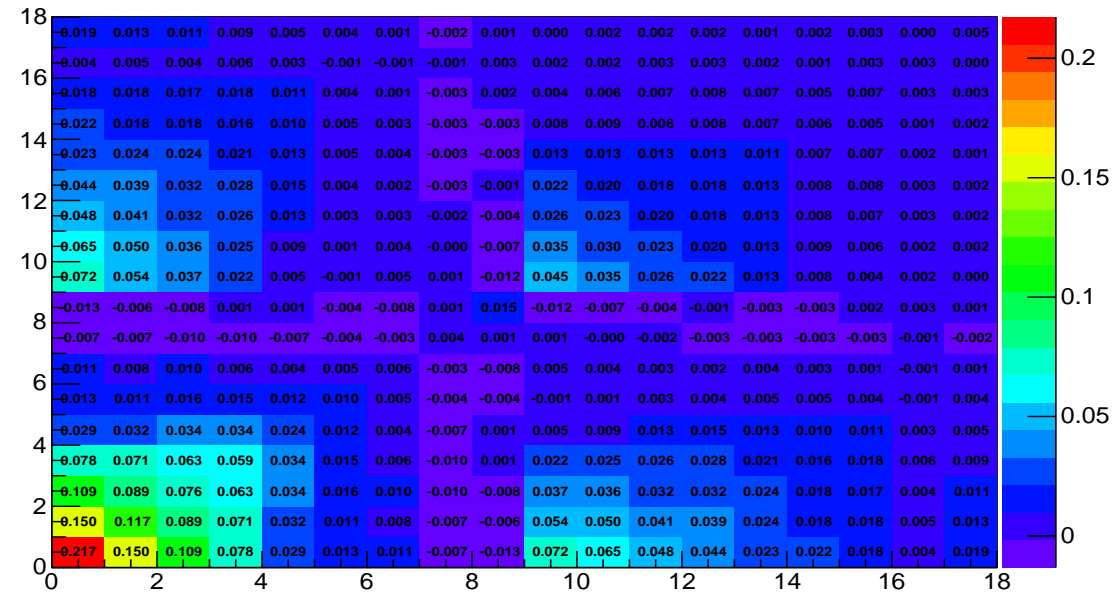

Figure 118: GENIE FSI uncertainty component of Covariance matrix for the extracted neutrino cross section in the FHC and antineutrino cross section in the RHC beam mode. The bin boundaries are in units of $\mathrm{GeV}$. The covariance elements are in units of $\left(10^{-38} \mathrm{~cm}^{2} / \mathrm{GeV}\right)^{2}$ and scaled by a factor of 1000. Index 1-9 are for neutrino cross section and 10-18 are for antineutrino cross section. 


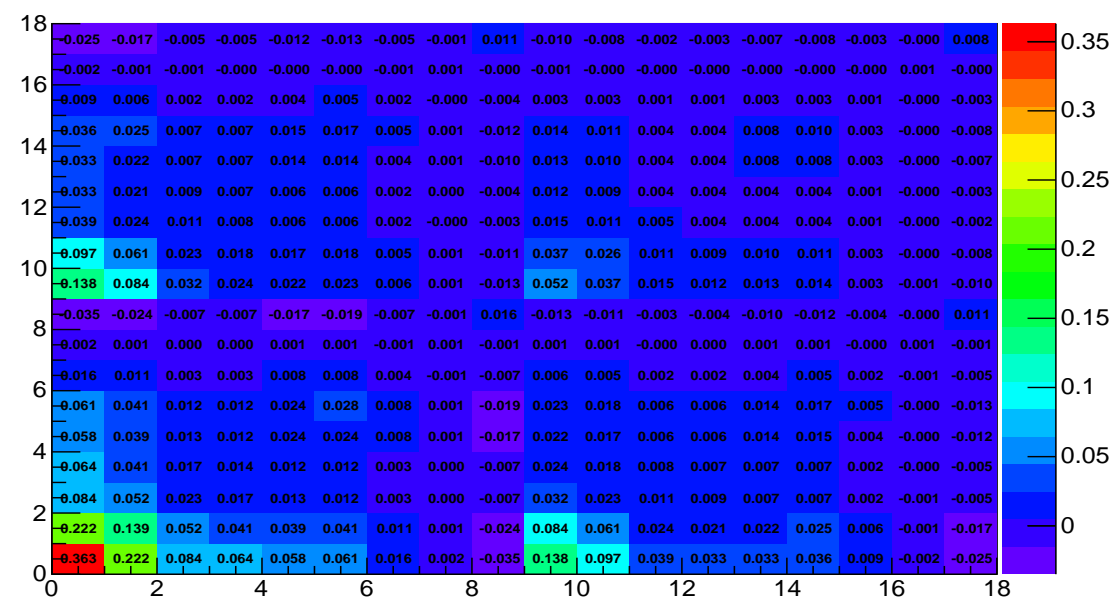

Figure 119: Hadron energy scale component of Covariance matrix for the extracted neutrino cross section in the FHC and antineutrino cross section in the RHC beam mode. The bin boundaries are in units of $\mathrm{GeV}$. The covariance elements are in units of $\left(10^{-38} \mathrm{~cm}^{2} / \mathrm{GeV}\right)^{2}$ and scaled by a factor of 1000. Index 1-9 are for neutrino cross section and 10-18 are for antineutrino cross section.

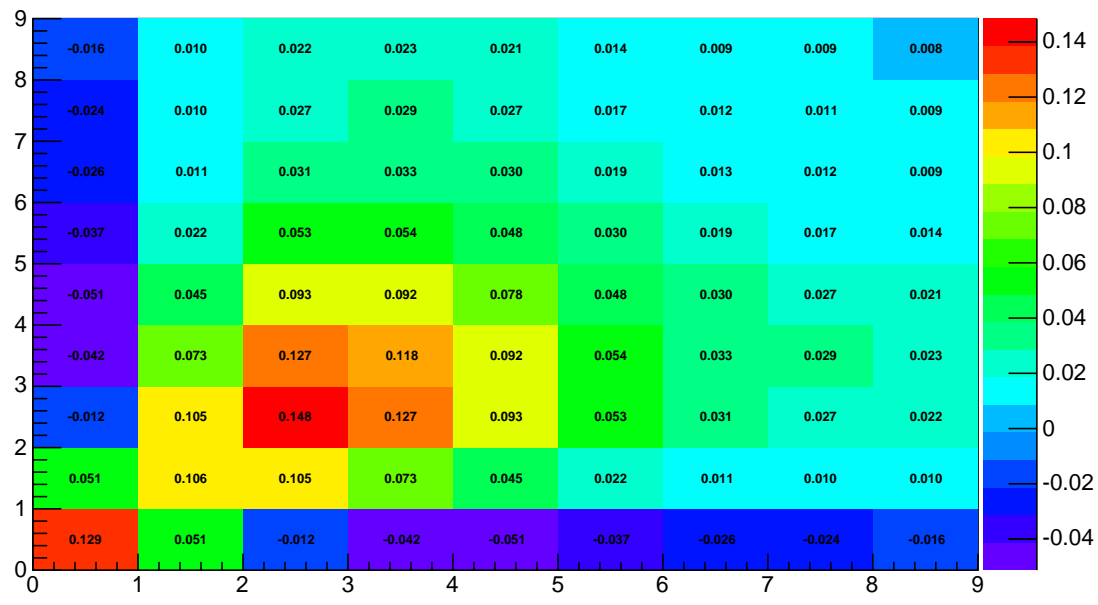

Figure 120: Components coming from GENIE cross section model uncertainty for the ratio. The bin boundaries are in units of $\mathrm{GeV}$. The covariance elements are scaled by a factor of 1000 . 


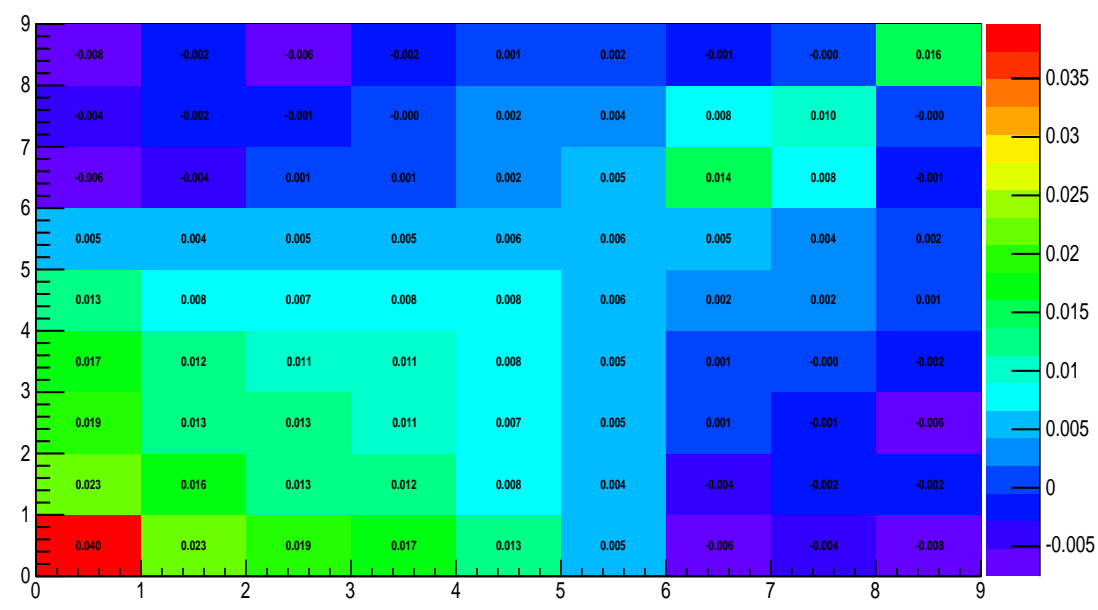

Figure 121: Components coming from GENIE FSI uncertainty for the ratio. The bin boundaries are in units of $\mathrm{GeV}$. The covariance elements are scaled by a factor of 1000 .

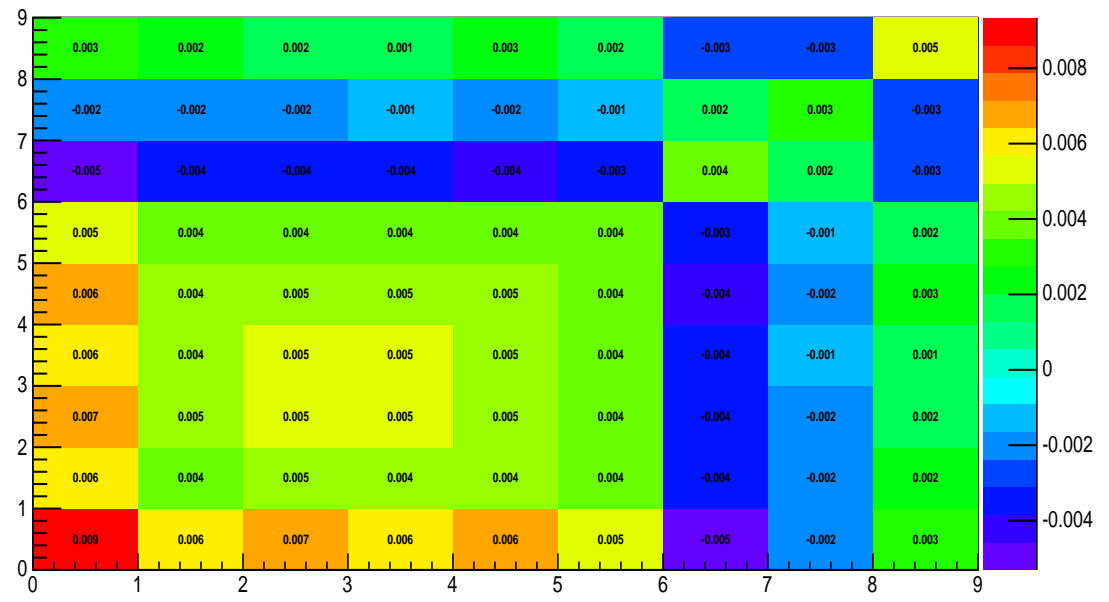

Figure 122: Components coming from muon energy scale for the ratio. The bin boundaries are in units of $\mathrm{GeV}$. The covariance elements are scaled by a factor of 1000 . 


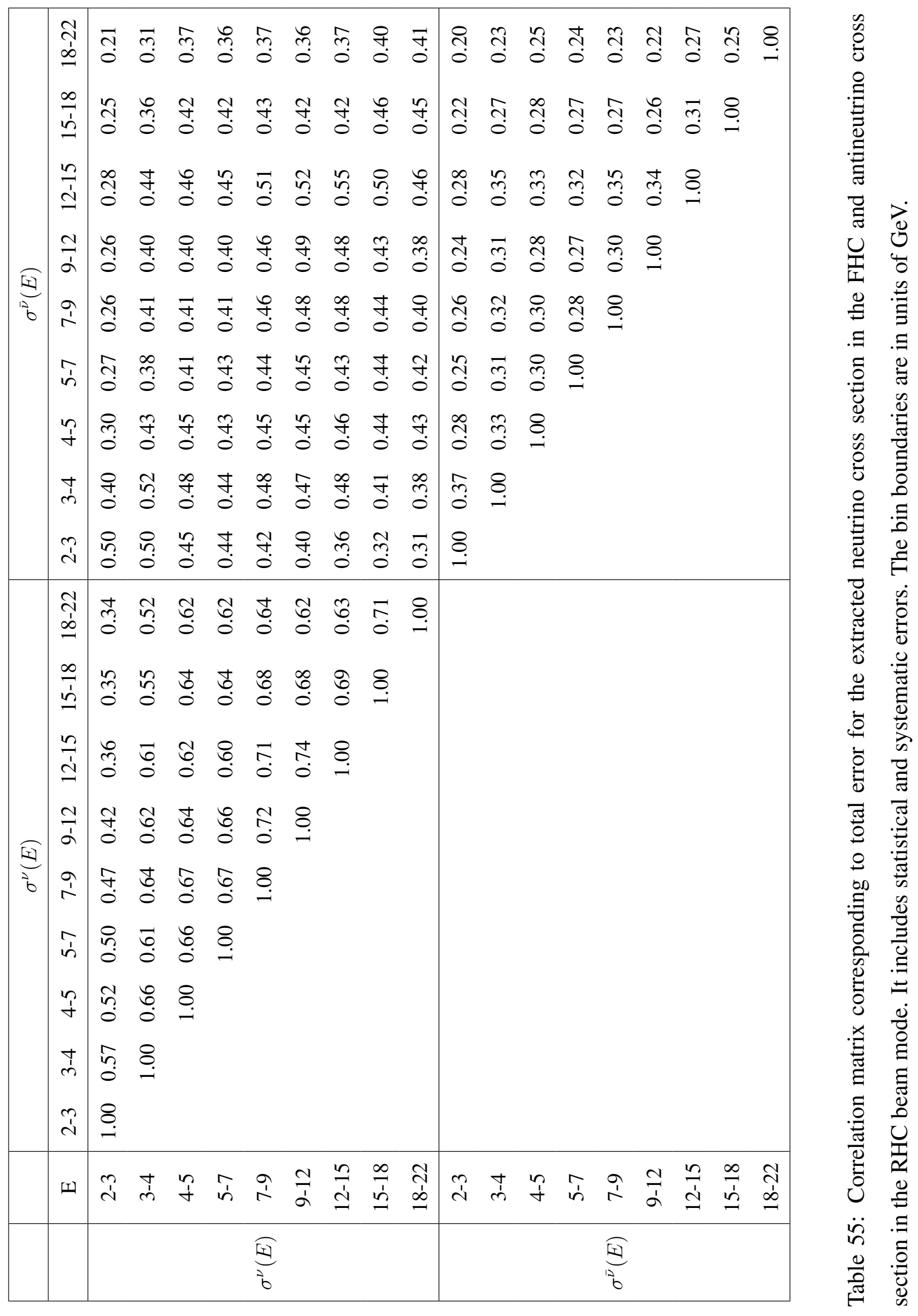




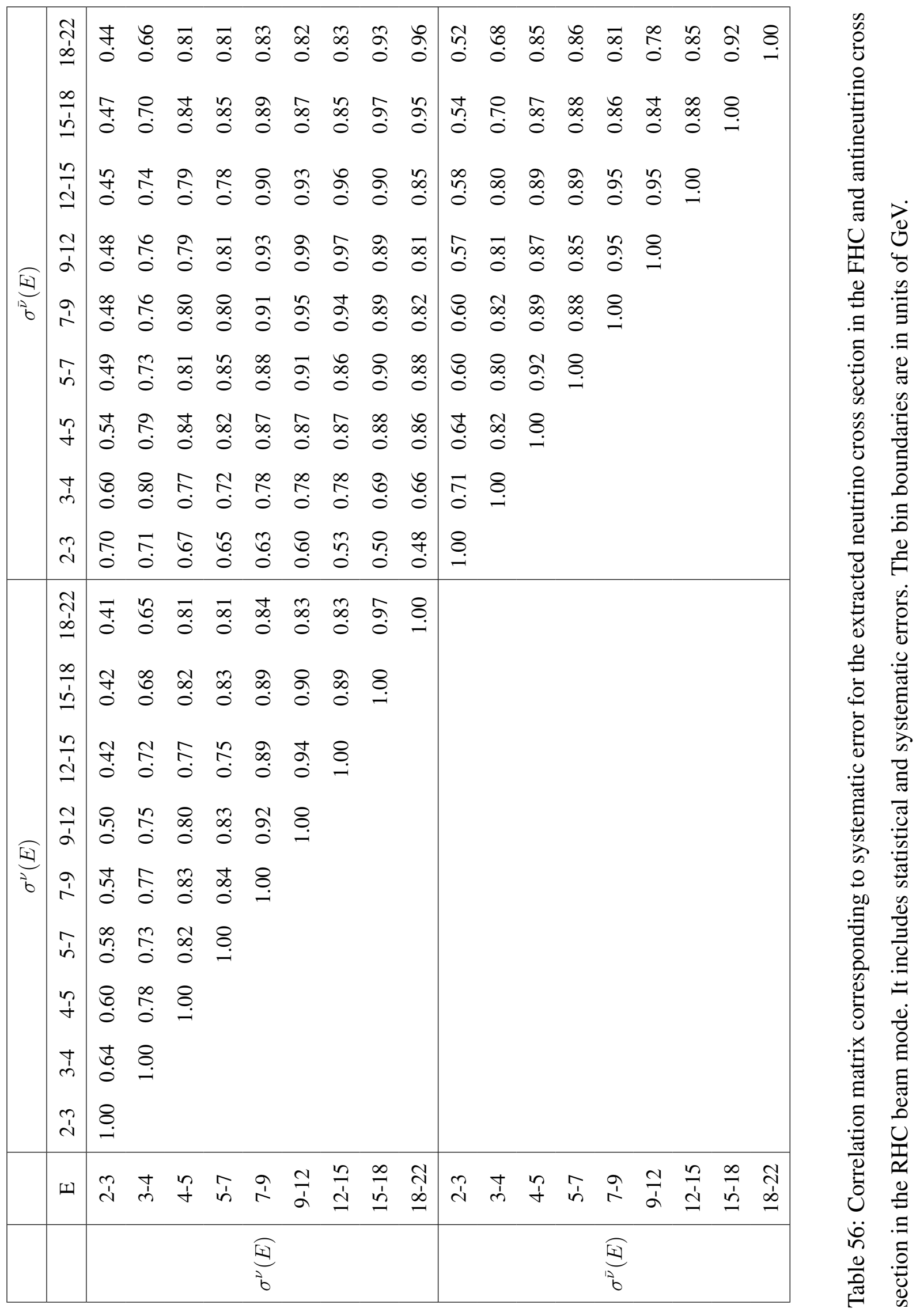




\section{B.3 $\chi^{2}$ CALCULATION FOR CROSS SECTIONS AND THE RATIO}

Using the covariance matrix we get in the previous section, we define $\chi^{2}$ as

$$
\chi^{2}=\sum_{i, i \leq j}^{N}\left(G_{i}-L_{i}\right)\left(L_{j}-G_{j}\right) V_{i j}^{-1},
$$

where $G_{i}$ is the GENIE-Hybrid model in energy bin $i$ and $L_{i}$ is measured quantity from data in the same bin. The matrix $V$ is the covariance matrices from previous section. The $\chi^{2}$ calculated is shown in Tab. 57.

\begin{tabular}{|c|c|c|c|}
\hline & Neutrino & Antineutrino & $r$ \\
\hline $\mathrm{NDF}$ & 9 & 9 & 9 \\
$\chi^{2}$ (full) & 20.4 & 5.03 & 1.44 \\
$\chi^{2}$ (diagonal) & 13.1 & 4.48 & 1.53 \\
\hline
\end{tabular}

Table 57: Calculated $\chi^{2}$ for neutrino and antineutrino cross sections and the ratio, $r$, between the GENIE-Hybrid model and the data. 


\section{APPENDIX C}

\section{CODE OF CALCULATING CROSS SECTIONS FROM TABLES}

The following piece of code is used for calculating cross sections from tables. data_inclusive_nu .dat (data_inclusive_nubar.dat) includes FHC neutrino (RHC antineutrino) data for inclusive samples. data_flux_nu.dat (data_flux_nubar.dat) includes data needed for normalization in normalization bin. correction_incl_nu.dat (correction_incl_nubar.dat) is table for model-dependent correction for FHC neutrinos (RHC antineutrinos). correction_flux_nu.dat (correction_flux_nubar.dat) is the table for model-dependent corrections in normalization bin for FHC neutrinos (RHC antineutrinos). 


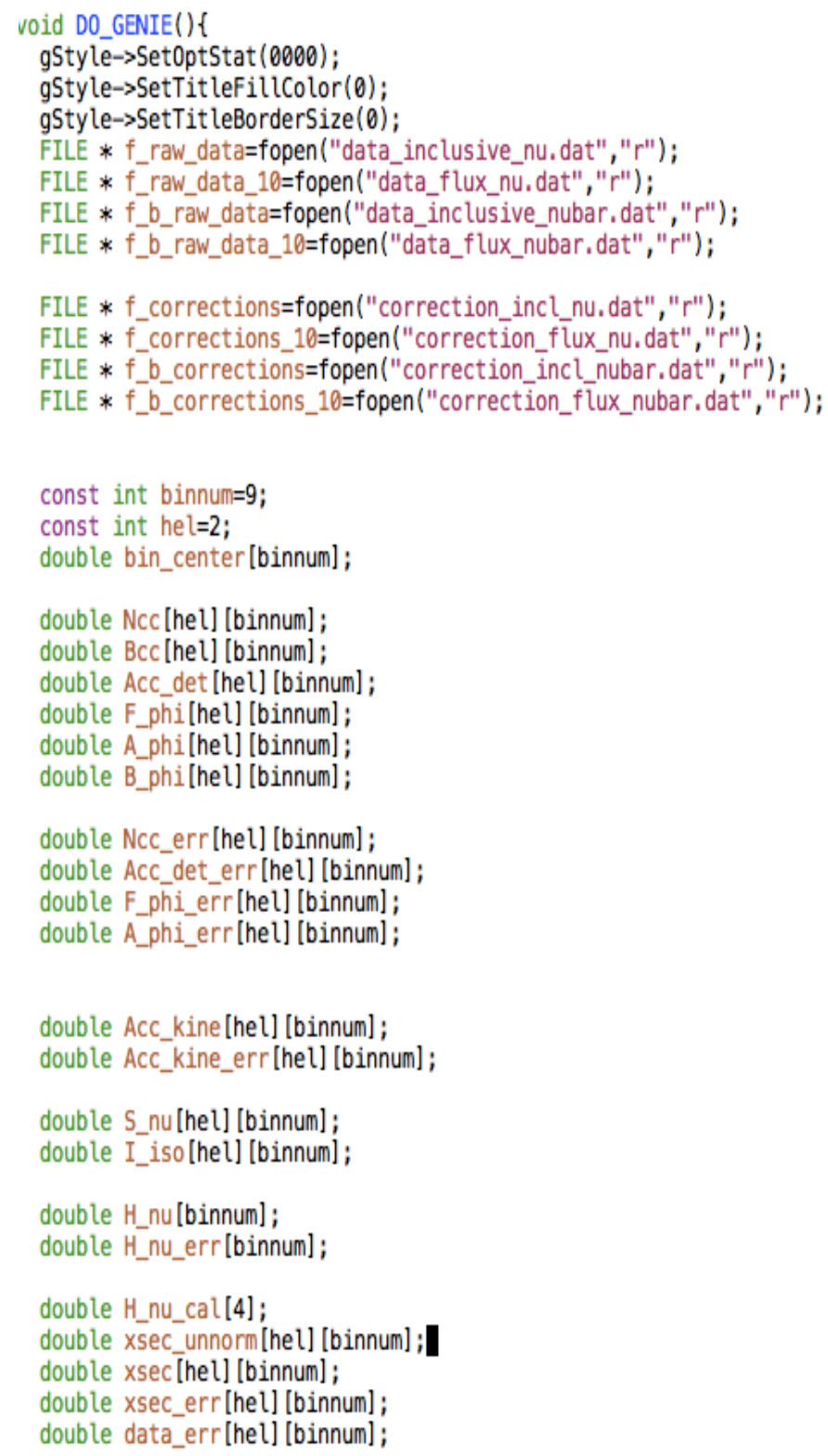




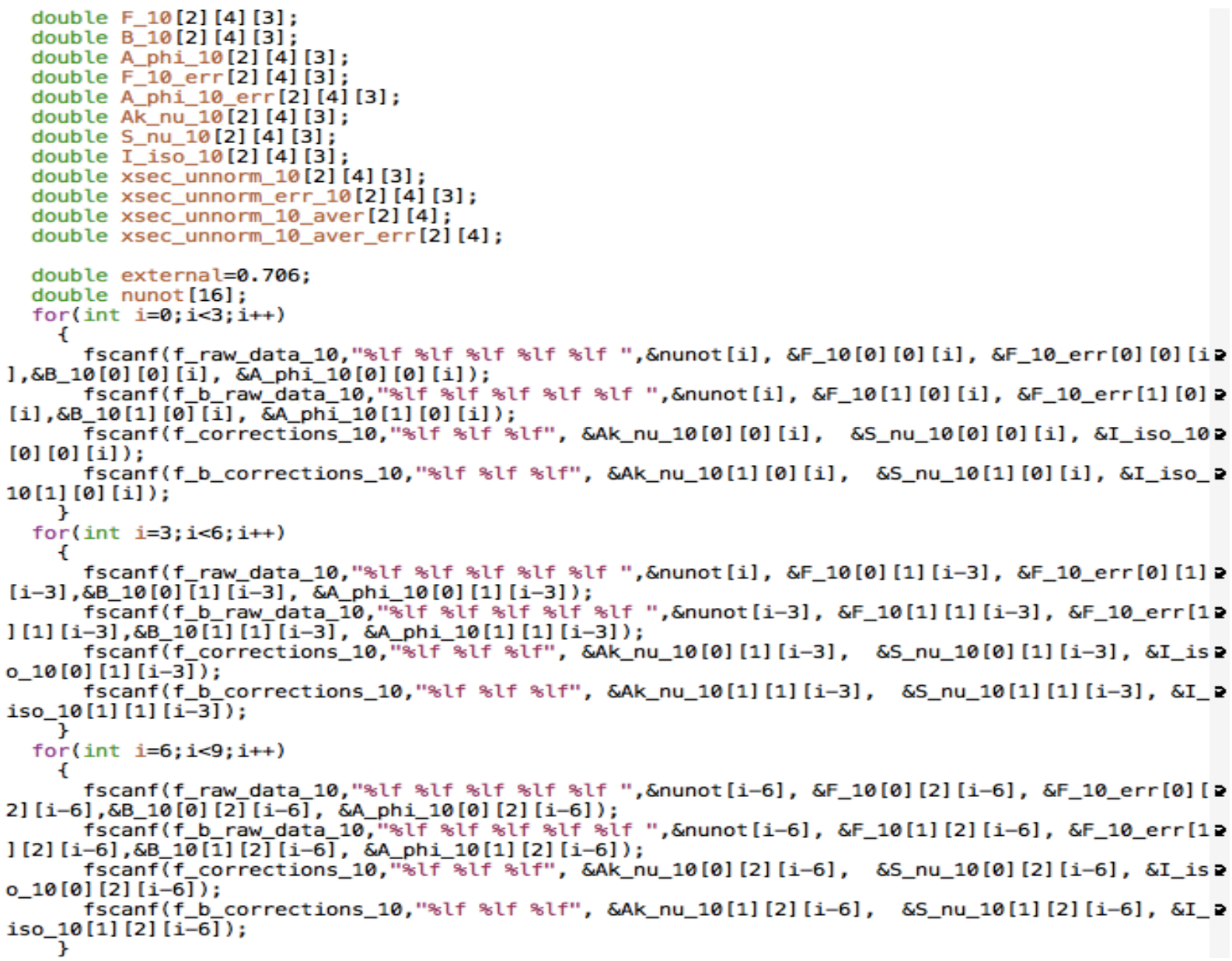


for (int $i=9 ; i<12 ; i++)$

\{

fscanf(f_raw_data_10,"slf slf slf slf slf ", \&nunot [i-9], \&F_10[0] [3] [i-9], \&F_10_err[0] [? 3] [i-9], \&B_10 [0] [3] [i-9], \&A_phi_10 [0] [3] [i-9]);

fscañf(f_b_raw_data_10,"slf slf slf slf siff ", \&nunot [i-9], \&F_10[1] [3] [i-9], \&F_10_err[1]

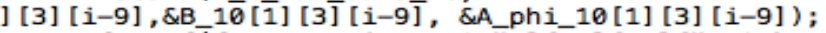

fscanf(f_corrections_10,"slf slf slf", \&Ak_nu_10[0] [3] [i-9], \&S_nu_10[0] [3] [i-9], \&I_is 2 0 -10[0][3] [i-9]):

fscanf(f_b_corrections_10,"slf slf slf", \&Ak_nu_10[1] [3] [i-9], \&S_nu_10[1] [3] [i-9], \&I_? iso_10 [1] [3] [i=9]);

\}

for(int $i=0 ; i<b i n n u m ; i++)$

\{

fscanf(f_raw_data,"slf slf slf slf slf slf slf slf slf ", \&bin_center[i], \&Ncc[0] [i], \&Nc?

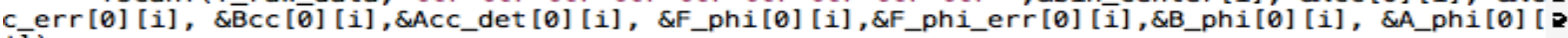
i]);

fscanf(f b raw data, "slf slf slf slf slf slf slf solf slf ", \&bin center[i], \&Ncc[1] [i], \& Ncc err[1] [i], \&Bcc[1][i], \&Acc_det[1][i], \&F_phi[1][i],\&F_phi_err[1][i], \&B_phi[1][i], \&A_phi, [1] $[$ i] );

f'scanf(f_corrections, "slf $s l f \xi_{\delta} f "$, \&Acc_kine[0] [i], \&S_nu[0] [i], \&I_iso[0] [i]);

fscanf(f_b_corrections, "slf slf slf ", \&Acc_kine[1] [i], \&S_nu[1] [i], \&I_iso[1] [i]);

$x \sec u n n o r m[0][i]=(N c c[0][i]-B c c[0][i]) * A c c \operatorname{det}[0][i] /\left(F \_p h i[0][i]-B\right.$ phi [0] [i] $) / A$ phi [0] 2 [i]*Acc kin̄e[0] [i] *S nu[0] [i]*I iso[0] [i]/bin center [i] :

$\times$ sec_unnorm[1] $[i]=(\operatorname{Ncc}[1] \overline{[}]-B c c[1][i]) * A c c \_\operatorname{det}[1][i] /\left(F \_p h i[1][i]-B \_p h i[1][i]\right) / A \_p h i[1] \geqslant$ [i]*Acc_kine [1] [i]*S_nu[1][i]*I_iso[1][i]/bin_center[i];

double sum weight nu[4]=\{0\};

double sum $x$ weight nu $[4]=\{\dot{\theta}\}$;

double $x$ ave $\bar{r}$ _nu [4];

double sigma_aver_nú[4];

double weight nu[4] [3];

double weight_nubar[4][3]

double x_aver_nubar[4];

double sigma aver nubar [4]

double sum_weight_nubar $[4]=\{0\}$;

double sum_x_weight_nubar $[4]=\{\hat{\theta}\}$;

for (int $i=0 ; i<4 ; i++)$

\{

for (int $j=0 ; j<3 ; j++$ )

$x$ sec_unnorm_10[0] [i] $[j]=(\mathrm{Ncc}[0][j+6]-B c c[0][j+6]) * A c c \_\operatorname{det}[0][j+6] /\left(\mathrm{F} \_10[0][i][j]-B=\right.$

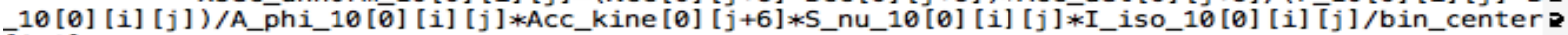
$\overline{[}]+6]$;

$\times \sec u n n o r m 10[1][i][j]=(\operatorname{Ncc}[1][j+6]-B c c[1][j+6]) * A c c \operatorname{det}[1][j+6] /(F-10[1][i][j]-B P$

_10[1][i][j])/A_phi_10[1][i][j]*ACC_kine[1][j+6]*S_nu_10[1][i][j]*I_iso_10[1][i][j]/bin_center? 
$[j+6] ;$

xsec_unnorm_err_10[0] [i] [j] $=\left(F_{-} \_10 \_e r r[0][i][j]\right.$-Ncc_err $\left.[0][j+6]\right) / 100$;

xsec_unnorm_err_10[1] [i] [j] =(F_10_err[1] [i] [j]-Ncc_err [1] $[j+6]) / 100$;

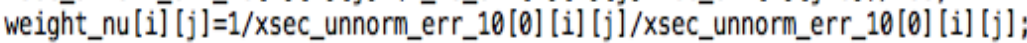

weight_nubar [i] [j] =1/xsec_unnorm_err_10[1] [i] [j]/xsec_unnorm_err_10[1] [i] [j] ; sum_weight_nu[i]+=weight_nu[i] [j];

sum_weight_nubar[i]+=weight_nubar [i] [j];

sum_x_weight_nu[i] +=xsec_unnorm_10[0] [i] [j] *weight_nu [i] [j] ;

\}

sum_x_weight_nubar [i]+=xsec_unnorm_10[1] [i] [j] *weight_nubar [i] [j] ;

x_aver_nu[i]=sum_x_weight_nu[i]/sum_weight_nu[i] ;

X_aver_nubar[i]=sum_x_weight_nubar[i] /sum_weight_nubar[i];

sigma_aver_nu[i] $=1 /$ squrt (sum_weight_nu[i]);

sigma_aver_nubar [i]=1/sqrt(sum_weight_nubar[i]);

\}

double norm_nu[binnum];

double norm_nubar [binnum];

double G_corr $[4]=\{1,1.004,1.0147,1.0266\}$;

for (int $i=0 ; i<b i n n u m ; i++)$

\{

if $(i==0)$

\{norm_nu $[i]=0.699 / x_{-}$aver_nu [0];

norm_nubar[i] =norm_nu [i] $* G$ _corr [0]*(1-( x_aver_nu[3]/x_aver_nu[0]- $x_{-}$aver_nubar [3] $\mathbf{p}$

*G_corr [3]/x_aver_nubar[0]/G_corr[0])/(x_aver_nu [3]/x_aver_nu [0])); $\}$

if $($ i>0 $>0<i<4)$

\{norm_nu [i] $=0.699 / x_{-}$aver_nu [1];

norm_nubar [i] =norm_nu [i] $* G_{-}$corr [1] $*\left(1-\left(x_{-}\right.\right.$aver_nu [3] $/ x_{-}$aver_nu [1] $-x_{-}$aver_nubar [3] 2

*G_corr [3]/x_aver_nubar[1]/G_corr[1])/(x_aver_nu[3]/x_aver_nu[1]));

if $(i>4 \delta \& \bar{i}<6)$

\{norm_nu [i] $=0.699 / x_{-}$aver_nu [2] ;

norm_nubar[i] =norm_nu[i] *G_corr [2]*(1-( x_aver_nu [3]/X_aver_nu[2]- x_aver_nubar [3] $\mathbf{p}$

*G_corr [3]/x_aver_nubar [2]/G_corr [2])/(x_aver_nu[3]/x_aver_nu[2]));

if $(i>=6)$

\{norm_nu $[i]=0.699 / x_{-}$aver_nu[3];

norm_nubar [i]=norm_nu [i] $*$ G_corr [3] ; $\}$

xsec $[\overline{0}][i]=x s e c \_u n n o r m[0][i]$ *norm_nu [i] ;

xsec [1] [i] =xsec_unnorm [1] [i]*norm_nubar [i];

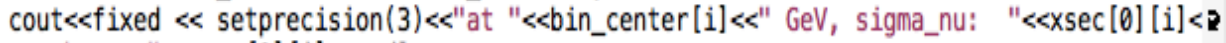

$<"$, sigma_nubar: "«<xec[1] [i] «<endl;

\}

\} 


\section{BIBLIOGRAPHY}

[1] Costas Andreopoulos, Christopher Barry, Steve Dytman, Hugh Gallagher, Tomasz Golan, Robert Hatcher, Gabriel Perdue, and Julia Yarba. The GENIE Neutrino Monte Carlo Generator: Physics and User Manual. 2015.

[2] Ivan Esteban, M. C. Gonzalez-Garcia, Michele Maltoni, Ivan Martinez-Soler, and Thomas Schwetz. Updated fit to three neutrino mixing: exploring the accelerator-reactor complementarity. JHEP, 01:087, 2017. doi: 10.1007/JHEP01(2017)087.

[3] R. Acciarri et al. Long-Baseline Neutrino Facility (LBNF) and Deep Underground Neutrino Experiment (DUNE). 2016.

[4] C. Patrignani et al. Review of Particle Physics. Chin. Phys., C40(10):100001, 2016. doi: 10.1088/1674-1137/40/10/100001.

[5] P. Adamson et al. The NuMI Neutrino Beam. Nucl. Instrum. Meth., A806:279-306, 2016. doi: 10.1016/j.nima.2015.08.063.

[6] Alexander I. Himmel. Antineutrino Oscillations in the Atmospheric Sector. PhD thesis, Caltech, 2011. URL http://lss.fnal.gov/cgi-bin/find_paper.pl? thesis-2011-13.

[7] Leonidas Aliaga Soplin. Neutrino Flux Prediction for the NuMI Beamline. PhD thesis, Coll. William and Mary, 2016. URL http://inspirehep. net/record/1465393/ files/fermilab-thesis-2016-07.pdf.

[8] L. Aliaga et al. Design, Calibration, and Performance of the MINERvA Detector. Nucl. Instrum. Meth., A743:130-159, 2014. doi: 10.1016/j.nima.2013.12.053.

[9] J. Nieves, Jose Enrique Amaro, and M. Valverde. Inclusive quasi-elastic neutrino reactions. Phys. Rev., C70:055503, 2004. doi: 10.1103/PhysRevC.70.055503,10.1103/PhysRevC.72. 019902. [Erratum: Phys. Rev.C72,019902(2005)].

[10] J. Nieves, I. Ruiz Simo, and M. J. Vicente Vacas. Inclusive Charged-Current NeutrinoNucleus Reactions. Phys. Rev., C83:045501, 2011. doi: 10.1103/PhysRevC.83.045501.

[11] Jaroslaw A. Nowak. Wroclaw neutrino event generator. Phys. Scripta, T127:70-72, 2006. doi: 10.1088/0031-8949/2006/T127/025. 
[12] A. Bodek, H. S. Budd, and M. E. Christy. Neutrino Quasielastic Scattering on Nuclear Targets: Parametrizing Transverse Enhancement (Meson Exchange Currents). Eur. Phys. J., C71:1726, 2011. doi: 10.1140/epjc/s10052-011-1726-y.

[13] P. A. Rodrigues et al. Identification of nuclear effects in neutrino-carbon interactions at low three-momentum transfer. Phys. Rev. Lett., 116:071802, 2016. doi: 10.1103/PhysRevLett. 116.071802 .

[14] P. Rodrigues. MINERvA Internal Document 11977. URL http://minerva-docdb . fnal.gov:8080/cgi-bin/ShowDocument?docid=11642.

[15] B. Pontecorvo. Inverse beta processes and nonconservation of lepton charge. Sov. Phys. JETP, 7:172-173, 1958. [Zh. Eksp. Teor. Fiz.34,247(1957)].

[16] B. Pontecorvo. Neutrino Experiments and the Problem of Conservation of Leptonic Charge. Sov. Phys. JETP, 26:984-988, 1968. [Zh. Eksp. Teor. Fiz.53,1717(1967)].

[17] Ziro Maki, Masami Nakagawa, and Shoichi Sakata. Remarks on the unified model of elementary particles. Prog. Theor. Phys., 28:870-880, 1962. doi: 10.1143/PTP.28.870.

[18] Debdatta Bhattacharya. Neutrino and antineutrino inclusive charged-current cross section measurement with the MINOS near detector. PhD thesis, Pittsburgh U., 2009. URL http: //lss.fnal.gov/cgi-bin/find_paper.pl?thesis-2009-11.

[19] Q. R. Ahmad et al. Measurement of the rate of $\nu_{e}+d \rightarrow p+p+e^{-}$interactions produced by ${ }^{8} B$ solar neutrinos at the Sudbury Neutrino Observatory. Phys. Rev. Lett., 87:071301, 2001. doi: 10.1103/PhysRevLett.87.071301.

[20] Hiroshi Nunokawa, Stephen J. Parke, and Jose W. F. Valle. CP Violation and Neutrino Oscillations. Prog. Part. Nucl. Phys., 60:338-402, 2008. doi: 10.1016/j.ppnp.2007.10.001.

[21] A Bodek and U. K. Yang. Higher twist, xi(omega) scaling, and effective LO PDFs for lepton scattering in the few GeV region. J. Phys., G29:1899-1906, 2003. doi: 10.1088/0954-3899/ 29/8/369.

[22] Richard P. Feynman. Very high-energy collisions of hadrons. Phys. Rev. Lett., 23:1415-1417, 1969. doi: 10.1103/PhysRevLett.23.1415.

[23] C. G. Callan and David J. Gross. High-energy electroproduction and the constitution of the electric current. Phys. Rev. Lett., 22:156-159, Jan 1969. doi: 10.1103/PhysRevLett.22.156. URL http://link.aps.org/doi/10.1103/PhysRevLett.22.156.

[24] K. A. Olive et al. Review of Particle Physics. Chin. Phys., C38:090001, 2014. doi: 10.1088/ 1674-1137/38/9/090001.

[25] R. P. Feynman, M. Kislinger, and F. Ravndal. Current matrix elements from a relativistic quark model. Phys. Rev., D3:2706-2732, 1971. doi: 10.1103/PhysRevD.3.2706. 
[26] Dieter Rein and Lalit M. Sehgal. Neutrino Excitation of Baryon Resonances and Single Pion Production. Annals Phys., 133:79-153, 1981. doi: 10.1016/0003-4916(81)90242-6.

[27] C. H. Llewellyn Smith. Neutrino Reactions at Accelerator Energies. Phys. Rept., 3:261-379, 1972. doi: 10.1016/0370-1573(72)90010-5.

[28] S. J. Barish et al. Inclusive Neutrino $\mathrm{p}$ and Neutrino n Charged-Current Neutrino Reactions Below 6-GeV. Phys. Lett., B66:291-294, 1977. doi: 10.1016/0370-2693(77)90883-8.

[29] S. J. Barish et al. Study of Neutrino Interactions in Hydrogen and Deuterium: Inelastic Charged Current Reactions. Phys. Rev., D19:2521, 1979. doi: 10.1103/PhysRevD.19.2521.

[30] C. Baltay et al. CROSS-SECTIONS AND SCALING VARIABLE DISTRIBUTIONS OF NEUTRAL AND CHARGED CURRENT NEUTRINO NUCLEON INTERACTIONS FROM A LOW-ENERGY NARROW BAND BEAM. Phys. Rev. Lett., 44:916-919, 1980. doi: 10.1103/PhysRevLett.44.916.

[31] N. J. Baker, P. L. Connolly, S. A. Kahn, M. J. Murtagh, R. B. Palmer, N. P. Samios, and M. Tanaka. Total Cross-sections for Muon-neutrino $N$ and Muon-neutrino $P$ Charged Current Interactions in the 7-ft Bubble Chamber. Phys. Rev., D25:617-623, 1982. doi: 10.1103/ PhysRevD.25.617.

[32] T. Eichten et al. Measurement of the Neutrino - Nucleon Anti-neutrino - Nucleon Total Cross-sections. Phys. Lett., B46:274-280, 1973. doi: 10.1016/0370-2693(73)90702-8.

[33] S. Ciampolillo et al. Total Cross-section for Neutrino Charged Current Interactions at 3-GeV and 9-GeV. Phys. Lett., B84:281-284, 1979. doi: 10.1016/0370-2693(79)90303-4.

[34] D. S. Baranov et al. Measurements of the $\nu_{\mu} N$ Total Cross-section at 2-GeV - 30-GeV in Skat Neutrino Experiment. Phys. Lett., B81:255-257, 1979. doi: 10.1016/0370-2693(79)90536-7.

[35] A. I. Mukhin, V. F. Perelygin, K. E. Shestermanov, A. A. Volkov, A. S. Vovenko, and V. P. Zhigunov. Energy Dependence of Total Cross-sections for Neutrino and Anti-neutrino Interactions at Energies Below 35-GeV. Sov. J. Nucl. Phys., 30:528, 1979. [Yad. Fiz.30,1014(1979)].

[36] V. B. Anikeev et al. Total cross-section measurements for muon-neutrino, anti-muon-neutrino interactions in 3-GeV - 30-GeV energy range with IHEP-JINR neutrino detector. Z. Phys., C70:39-46, 1996. doi: 10.1007/s002880050078.

[37] Q. Wu et al. A Precise measurement of the muon neutrino-nucleon inclusive charged current cross-section off an isoscalar target in the energy range $2.5 ; \mathrm{E}(\mathrm{nu}) ; 40-\mathrm{GeV}$ by NOMAD. Phys. Lett., B660:19-25, 2008. doi: 10.1016/j.physletb.2007.12.027.

[38] P. Adamson et al. Neutrino and Antineutrino Inclusive Charged-current Cross Section Measurements with the MINOS Near Detector. Phys. Rev., D81:072002, 2010. doi: 10.1103/PhysRevD.81.072002. 
[39] Y. Nakajima et al. Measurement of inclusive charged current interactions on carbon in a fewGeV neutrino beam. Phys. Rev., D83:012005, 2011. doi: 10.1103/PhysRevD.83.012005.

[40] C. Anderson et al. First Measurements of Inclusive Muon Neutrino Charged Current Differential Cross Sections on Argon. Phys. Rev. Lett., 108:161802, 2012. doi: 10.1103/PhysRevLett. 108.161802.

[41] K. Abe et al. Measurement of the inclusive charged current cross section on carbon in the near detector of the T2K experiment. Phys. Rev., D87(9):092003, 2013. doi: 10.1103/ PhysRevD.87.092003.

[42] K. Abe et al. Measurement of the muon neutrino inclusive charged-current cross section in the energy range of $13 \mathrm{GeV}$ with the T2K INGRID detector. Phys. Rev., D93(7):072002, 2016. doi: 10.1103/PhysRevD.93.072002.

[43] G. Fanourakis, L. K. Resvanis, G. Grammatikakis, P. Tsilimigras, A. Vayaki, U. Camerini, W. F. Fry, R. J. Loveless, J. H. Mapp, and D. D. Reeder. Study of Low-energy Anti-neutrino Interactions on Protons. Phys. Rev., D21:562, 1980. doi: 10.1103/PhysRevD.21.562.

[44] A. E. Asratian et al. TOTAL ANTI-NEUTRINOS NUCLEON CHARGED CURRENT CROSS-SECTION IN THE ENERGY RANGE 10-GEV - 50-GEV. Phys. Lett., B137:122124, 1984. doi: 10.1016/0370-2693(84)91118-3.

[45] A. E. Asratian et al. CHARGED CURRENT NEUTRINO INTERACTIONS BELOW 30GeV. Phys. Lett., B76:239-242, 1978. doi: 10.1016/0370-2693(78)90286-1.

[46] R. Acciarri et al. Measurements of Inclusive Muon Neutrino and Antineutrino Charged Current Differential Cross Sections on Argon in the NuMI Antineutrino Beam. Phys. Rev., D89 (11):112003, 2014. doi: 10.1103/PhysRevD.89.112003.

[47] P. Adamson et al. First measurement of electron neutrino appearance in NOvA. Phys. Rev. Lett., 116(15):151806, 2016. doi: 10.1103/PhysRevLett.116.151806.

[48] V. Daniel Elvira, Paul Lebrun, and Panagiotis Spentzouris. Beam simulation tools for GEANT4 (and neutrino source applications). In 7th International Computational Accelerator Physics Conference (ICAP 2002) East Lasing, Michigan, October 15-18, 2002, pages 73-82, 2005. URL http://lss. fnal.gov/cgi-bin/find_paper.pl?conf-02-272.

[49] B. Baatar et al. Inclusive production of protons, anti-protons, neutrons, deuterons and tritons in $\mathrm{p}+\mathrm{C}$ collisions at $158 \mathrm{GeV} / \mathrm{c}$ beam momentum. Eur. Phys. J., C73(4):2364, 2013. doi: 10.1140/epjc/s10052-013-2364-3.

[50] L. Aliaga et al. MINERvA neutrino detector response measured with test beam data. Nucl. Instrum. Meth., A789:28-42, 2015. doi: 10.1016/j.nima.2015.04.003.

[51] D. G. Michael et al. The Magnetized steel and scintillator calorimeters of the MINOS experiment. Nucl. Instrum. Meth., A596:190-228, 2008. doi: 10.1016/j.nima.2008.08.003. 
[52] Tomasz Golan, Cezary Juszczak, and Jan T. Sobczyk. Final State Interactions Effects in Neutrino-Nucleus Interactions. Phys. Rev., C86:015505, 2012. doi: 10.1103/PhysRevC.86. 015505.

[53] Philip Rodrigues, Callum Wilkinson, and Kevin McFarland. Constraining the GENIE model of neutrino-induced single pion production using reanalyzed bubble chamber data. 2016.

[54] R. Bradford, A. Bodek, Howard Scott Budd, and J. Arrington. A New parameterization of the nucleon elastic form-factors. Nucl. Phys. Proc. Suppl., 159:127-132, 2006. doi: 10.1016/ j.nuclphysbps.2006.08.028. [,127(2006)].

[55] Konstantin S. Kuzmin, Vladimir V. Lyubushkin, and Vadim A. Naumov. Axial masses in quasielastic neutrino scattering and single-pion neutrinoproduction on nucleons and nuclei. Acta Phys. Polon., B37:2337-2348, 2006.

[56] D. Rein and L. M. Sehgal. PCAC and the Deficit of Forward Muons in pi+ Production by Neutrinos. Phys. Lett., B657:207-209, 2007. doi: 10.1016/j.physletb.2007.10.025.

[57] Dieter Rein and Lalit M. Sehgal. Coherent piO Production in Neutrino Reactions. Nucl. Phys., B223:29-44, 1983. doi: 10.1016/0550-3213(83)90090-1.

[58] S. G. Kovalenko. Quasielastic neutrino production of charmed baryons from the point of view of local duality. Sov. J. Nucl. Phys., 52:934-936, 1990. [Yad. Fiz.52,1478(1990)].

[59] M. A. G. Aivazis, Fredrick I. Olness, and Wu-Ki Tung. Leptoproduction of heavy quarks. i. general formalism and kinematics of charged current and neutral current production processes. Phys. Rev. D, 50:3085-3101, Sep 1994. doi: 10.1103/PhysRevD.50.3085. URL http://link.aps.org/doi/10.1103/PhysRevD.50.3085.

[60] D.Yu. Bardin and V.A. Dokuchaeva. Muon energy spectrum in inverse -decay. $N u$ clear Physics B, 287:839 - 851, 1987. ISSN 0550-3213. doi: http://dx.doi.org/10. 1016/0550-3213(87)90131-3. URL http: / /www.sciencedirect.com/science/ article/pii/0550321387901313.

[61] H. Gallagher. Using electron scattering data to tune neutrino Monte Carlos. AIP Conf. Proc., 698:153-157, 2004. doi: 10.1063/1.1664215. [,153(2004)].

[62] R. Gran, J. Nieves, F. Sanchez, and M. J. Vicente Vacas. Neutrino-nucleus quasi-elastic and 2p2h interactions up to $10 \mathrm{GeV}$. Phys. Rev., D88(11):113007, 2013. doi: 10.1103/PhysRevD. 88.113007 .

[63] L. Alvarez-Ruso, S. K. Singh, and M. J. Vicente Vacas. Charged current weak electroproduction of Delta resonance. Phys. Rev., C57:2693-2699, 1998. doi: 10.1103/PhysRevC.57.2693.

[64] N. Tagg et al. Arachne - A web-based event viewer for MINERvA. Nucl. Instrum. Meth., 676:44-49, 2012. doi: 10.1016/j.nima.2012.01.059. 
[65] R. Fruhwirth. Application of Kalman filtering to track and vertex fitting. Nucl. Instrum. Meth., A262:444-450, 1987. doi: 10.1016/0168-9002(87)90887-4.

[66] Ronald Ransome. MINERvA Internal Document 6016. URL http: //minerva-docdb . fnal.gov: $8080 /$ cgi-bin/ShowDocument.

[67] Joshua D. Devan. Measurement of Neutrino and Antineutrino Charged-Current Inclusive Cross Sections with the MINERvA Detector. $\mathrm{PhD}$ thesis, Coll. William and Mary, 2015. URL http://lss.fnal.gov/archive/thesis/2000/ fermilab-thesis-2015-29.pdf.

[68] M. Betancourt. MINERvA Internal Document 11642. URL http://minerva-docdb . fnal.gov:8080/cgi-bin/ShowDocument?docid=11642. 